\title{
Perspective LOBAU 2020
}

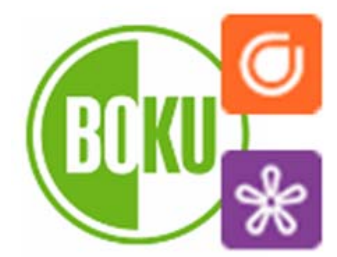

\section{Endbericht}

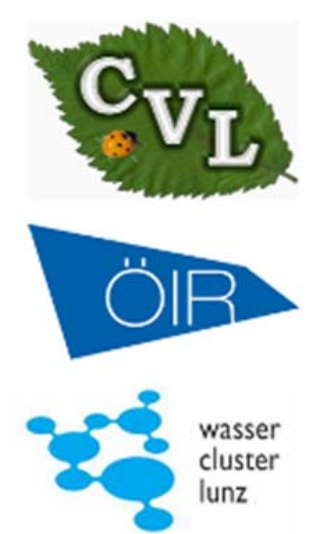

Exploring management options of a heavily used urban biosphere reserve confronted with new urban developments in its neighbourhood considering a restricted potential for ecosystem development

(Welche Perspektiven gibt es für die Lobau im Jahr 2020? Untersuchung von Managementoptionen für den stark besuchten Biosphärenpark aufgrund der Wiener Stadtentwicklung)

Arnberger, A., Deussner, R., Eder, R., Hein, T., Illedits, A., Kempter, I., Taczanowska, K., Nopp-Mayr, U., Preiner, S., Reiter, K., Stanzer, G., Wagner, I., Zsak, K.

Gefördert durch das „Man and Biosphere Programm“ der Österreichischen Akademie der Wissenschaften

April 2012

Online-ISBN: 978-3-7001-7288-8

DOI: 10.1553/Lobau2020 


\section{Projektpartner:}

Universität für Bodenkultur,

Institut für Landschaftsentwicklung, Erholungs- und Naturschutzplanung (ILEN); Dept. für Raum, Landschaft und Infrastruktur

arne.arnberger@boku.ac.at

Institut für Wildbiologie und Jagdwirtschaft (IWJ); Dept. für Integrative Biologie

iris.kempter@boku.ac.at

Universität Wien,

Fakultät für Lebenswissenschaften, Dept. für Naturschutzbiologie, Vegetations- und

Landschaftsökologie (CVL)

karl.reiter@univie.ac.at

WasserCluster Lunz - Biologische Station GmbH

thomas.hein@boku.ac.at

Österreichisches Institut für Raumplanung (ÖIR)

deussner@oir.at

\section{Ansprechpartner:}

Assoz. Prof. Dr. Arne Arnberger (ILEN): arne.arnberger@boku.ac.at

\section{Weitere Projektpartner:}

Forstamt der Stadt Wien, Magistratsabteilung 49, Nationalpark Forstverwaltung Lobau Nationalpark Donau-Auen GmbH

Alterra, Ing. Rene Jochem (Niederlande) 


\section{Inhaltsverzeichnis}

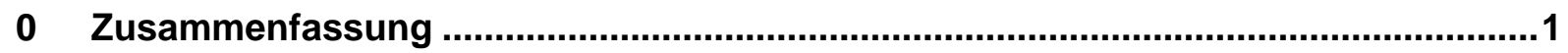

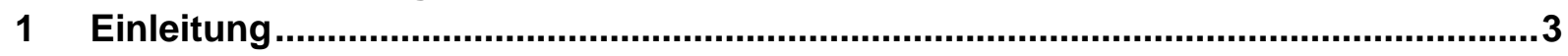

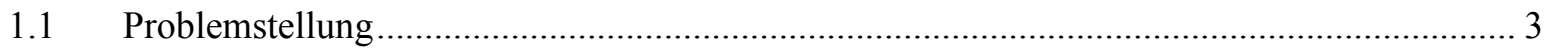

$1.2 \quad$ Projektziele

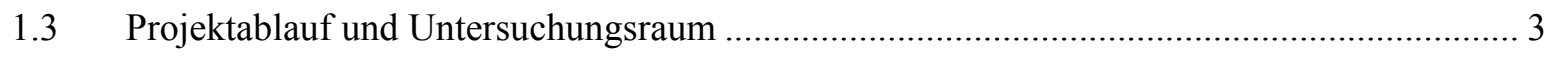

2 Prognose des Besucherdrucks auf die Lobau im Jahr 2025 aufgrund

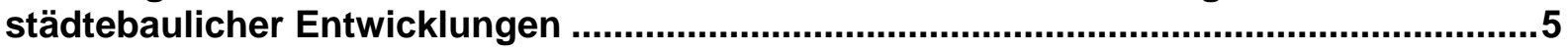

2.1 Grundlagen für die Prognose der Besuchshäufigkeiten ...................................................... 5

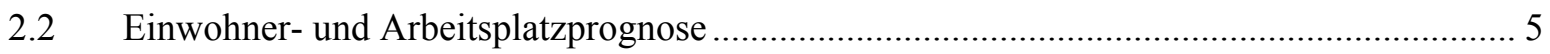

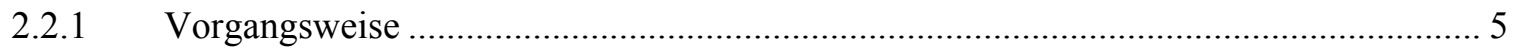

2.2.2 Masterplan ehemaliges Flugfeld Aspern (,Seestadt Aspern“) …..................................... 6

2.2.3 Einwohnerprognose übrige Bereiche ....................................................................... 7

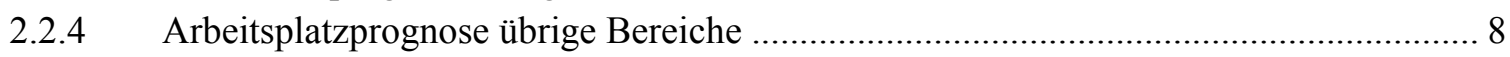

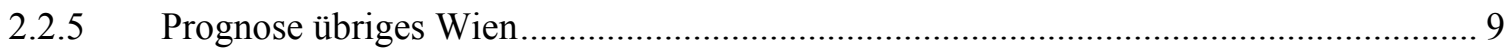

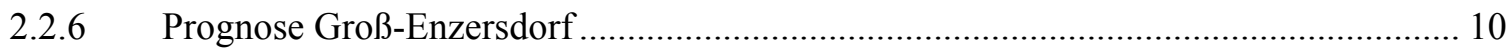

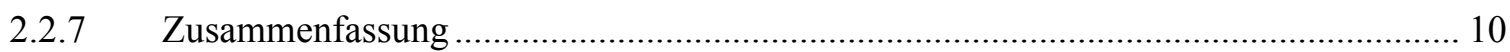

2.3 Prognostizierter Besucherdruck auf die Lobau im Jahr 2025 ............................................. 11

3 Szenarienentwicklung (Maßnahmenbündel) unter Einbezug von Stakeholdern ....13

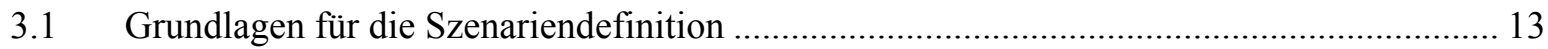

3.1.1 Maßnahmen aus gewässerökologischer Sicht in der Lobau ....................................... 13

3.1.2 Maßnahmen aus erholungsplanerischer Sicht im Lobau-Vorland................................ 13

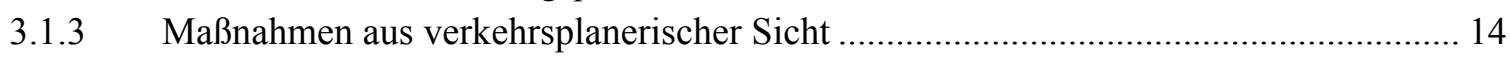

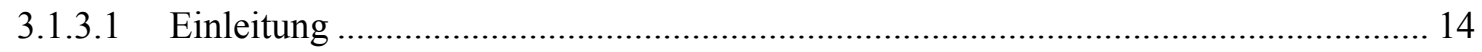

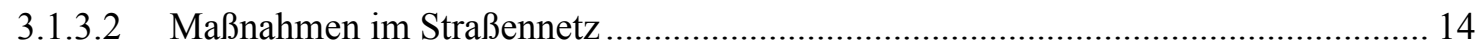

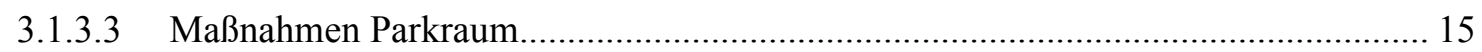

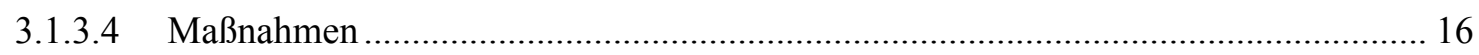

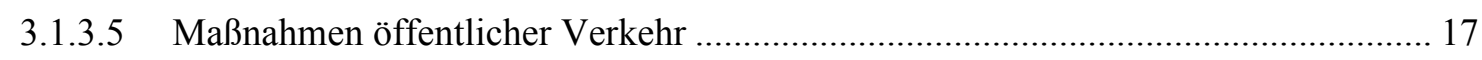

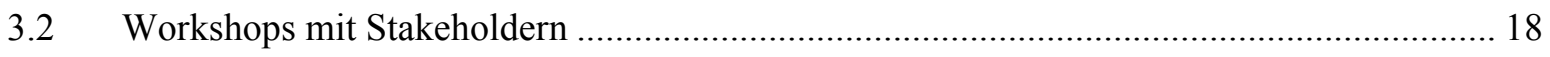

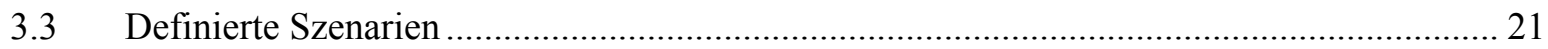

4 Bestimmung der Eingangsparameter als Basis für die Simulation des

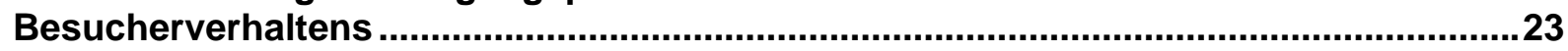

4.1 Modellierung des Besucherverhaltens anhand eines Wahlmodells..................................... 23

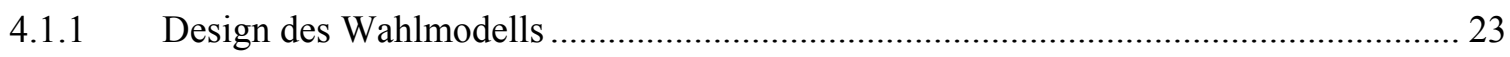

4.1.2 Erstellung des Erhebungsinstruments und des Wahlmodells .......................................26

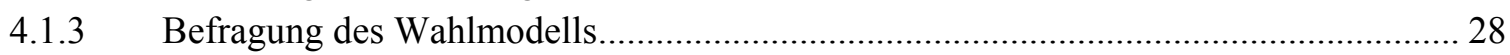

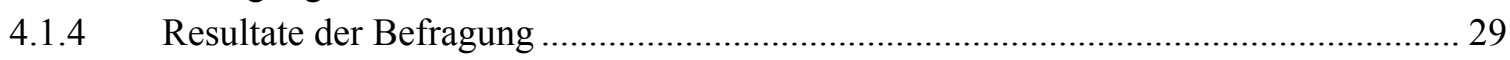

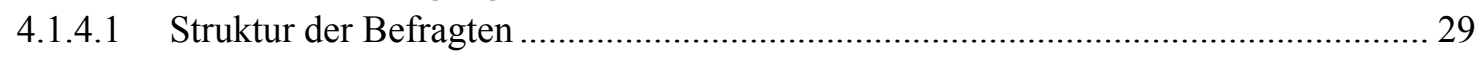

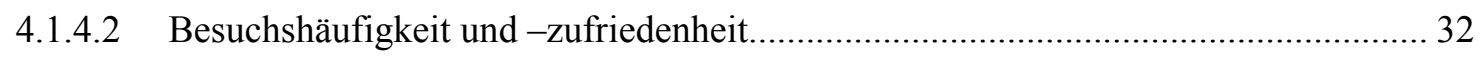

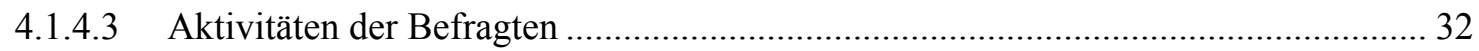

4.1.4.4 Auswertungen zu den Präferenzen der Erholungssuchenden auf Basis des

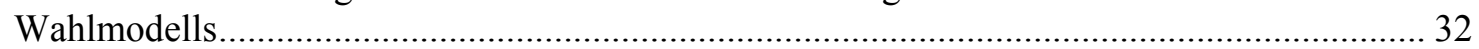

4.1.4.5 Heterogenität hinsichtlich Landschaftspräferenzen................................................... 34

4.2 Eingabeparameter zur Modellierung der räumlichen Verteilung der BesucherInnen in der

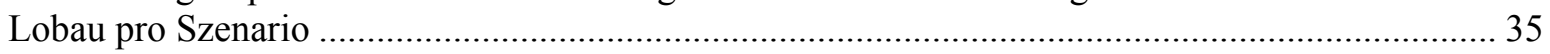

4.2.1 Agentenbasierte Modellierung des Besucherverhaltens - MASOOR Plattform........... 35

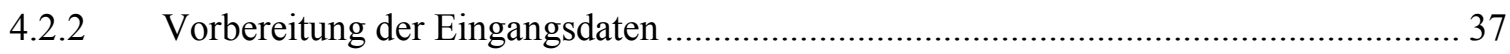

4.2.3 Definition der Eingabeparameter ........................................................................... 38 


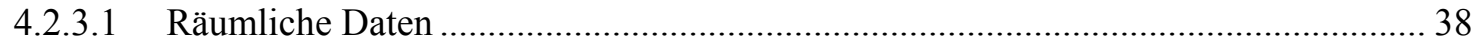

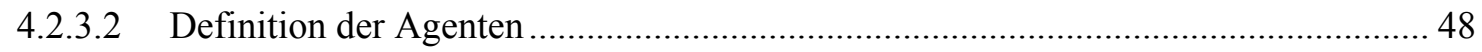

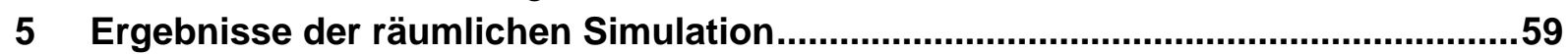

5.1 Räumliche Verteilung der BesucherInnen in der Lobau an einem Spitzenbesuchstag ......... 59

5.2 Räumliche Verteilung der BesucherInnen in der Lobau an einem Werktag ............................63 63

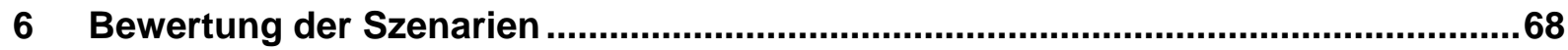

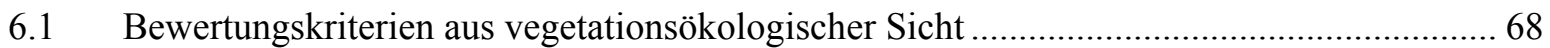

6.1.1 Bewertung des Einflusses einer steigenden Erholungsnutzung auf die Hemerobie...... 68

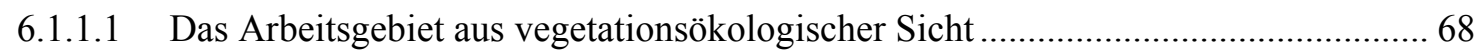

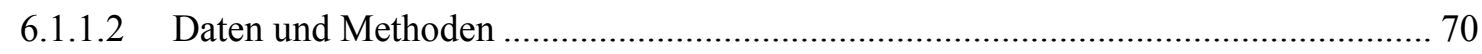

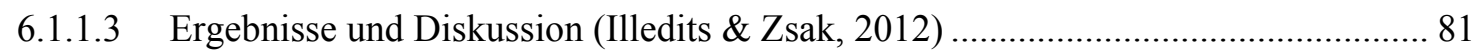

6.1.2 Bewertung des Einflusses einer steigenden Erholungsnutzung auf die Vegetationstypen

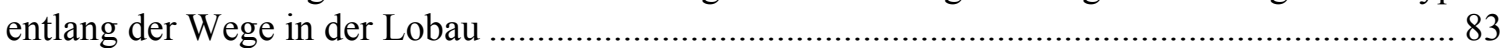

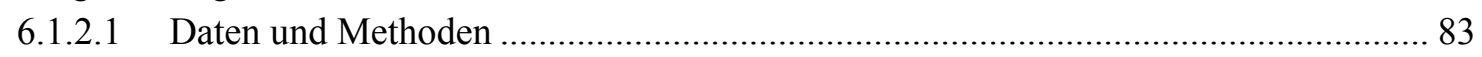

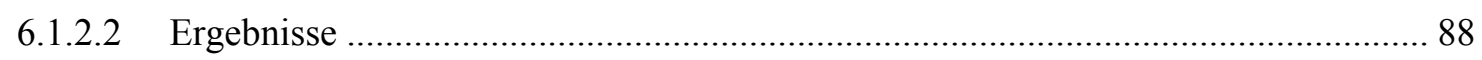

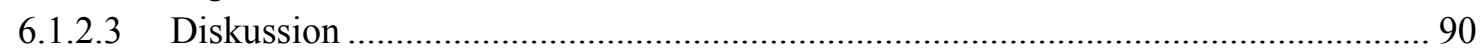

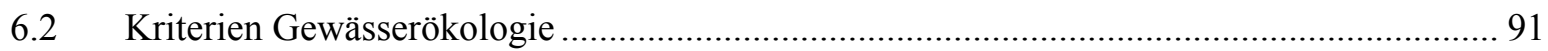

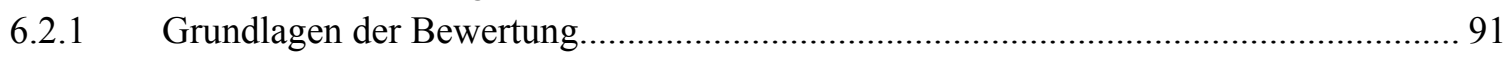

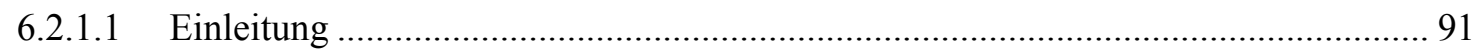

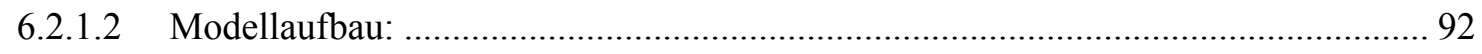

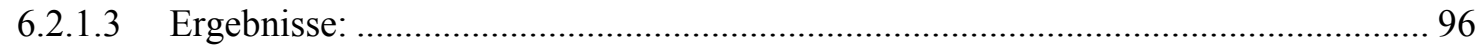

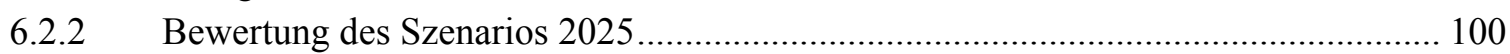

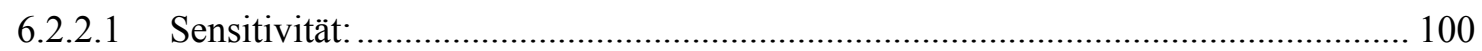

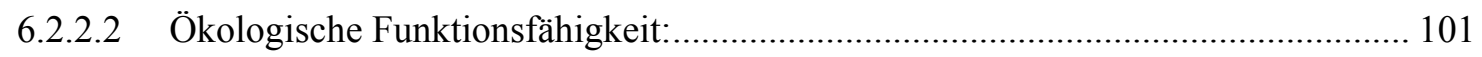

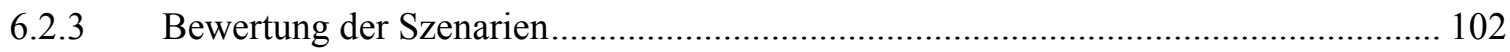

6.2.3.1 Gewässerszenario B - Sensitivität..................................................................... 102

6.2.3.2 Gewässerszenario B - ökologische Funktionsfähigkeit ....................................... 103

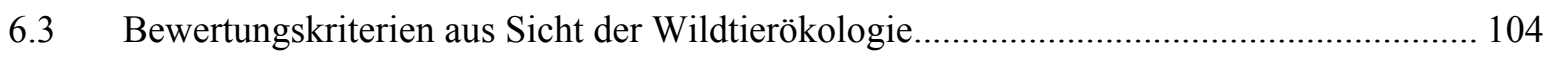

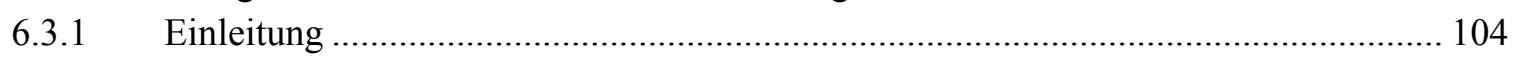

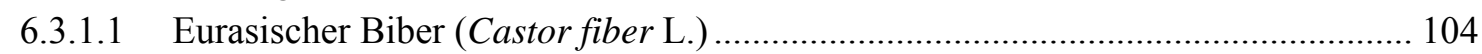

6.3.1.2 Reh- und Rotwild (Capreolus capreolus L. und Cervus elaphus L.)....................... 104

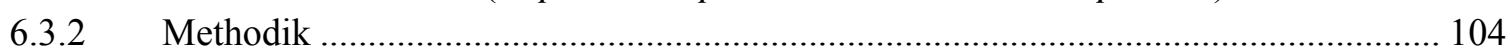

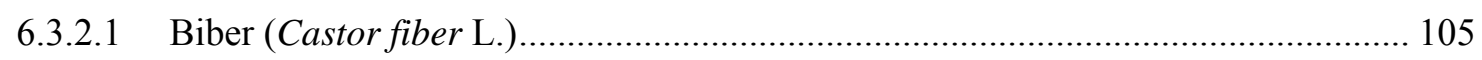

6.3.2.2 Reh- und Rotwild (Capreolus capreolus L. und Cervus elaphus L.)...................... 107

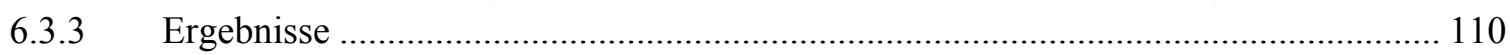

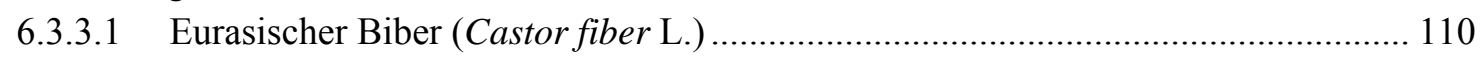

6.3.3.2 Reh- und Rotwild (Capreolus capreolus L. und Cervus elaphus L.) ........................ 112

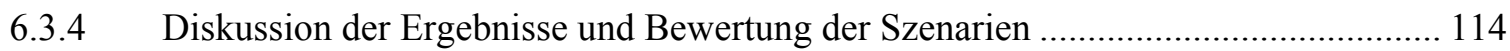

6.3.4.1 Eurasischer Biber (Castor fiber L.) .................................................................... 114

6.3.4.2 Reh- und Rotwild (Capreolus capreolus L. und Cervus elaphus L.)....................... 115

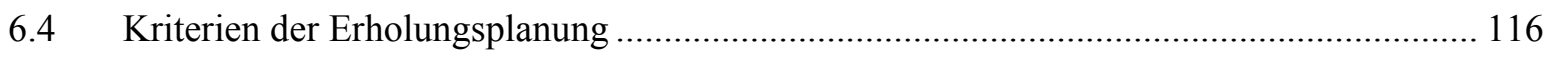

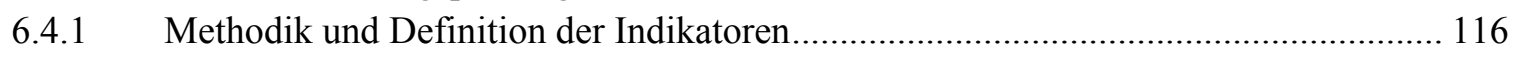

6.4.2 Auswirkungen des prognostizierten Besucherdrucks auf die Erholungsqualität.......... 118

6.4.3 Entlastung der Lobau durch die Pufferwirkung des Lobau-Vorlandes ........................ 119

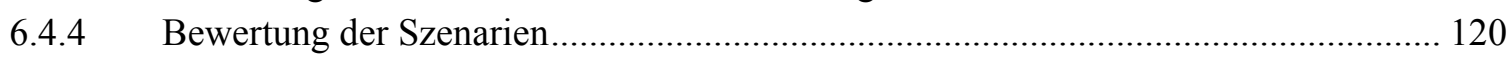

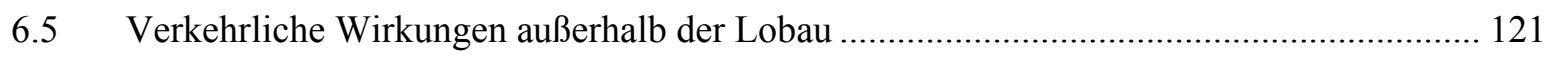

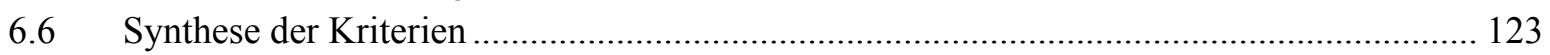

7 Diskussion und Vorschläge für Managementmaßnahmen ..................................129

7.1 Zusammenfassende Bewertungen der Szenarien ............................................................ 129 


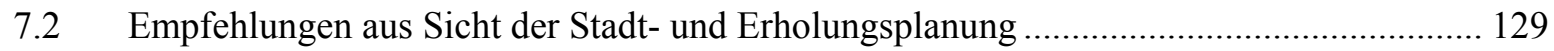

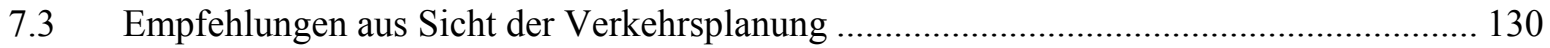

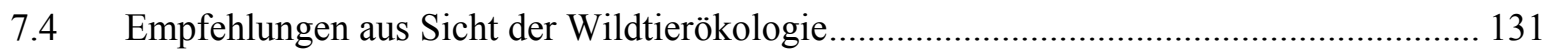

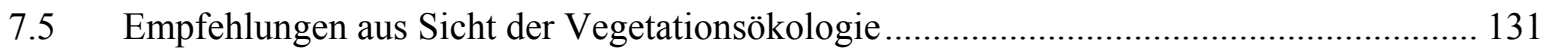

7.6 Empfehlungen aus Sicht der Gewässerökologie .......................................................... 132

7.7 Bedarf nach einem weiterführenden Biosphere Reserve Integrated Monitoring ................ 132

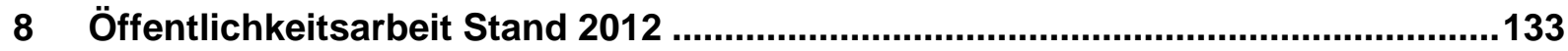

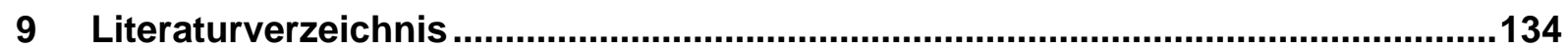

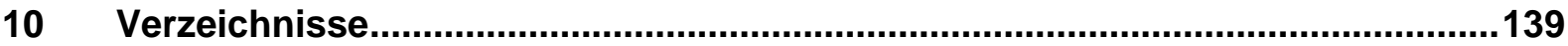

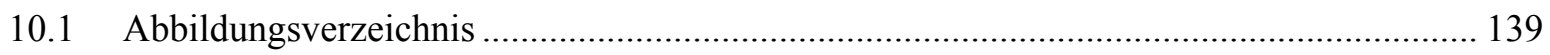

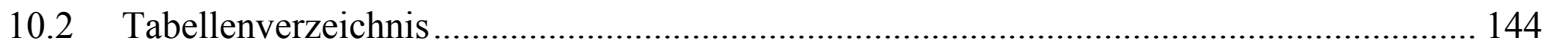




\section{Zusammenfassung}

Der Wiener UNESCO Biosphärenpark (BP) Untere Lobau ist ein durch Grundwasser und Rückstau gespeister Auwald und beherbergt ein äußerst vielfältiges Mosaik an aquatischen, halb-aquatischen und terrestrischen Habitaten. Gleichzeitig leben rund 150.000 Menschen in direkter Nachbarschaft zum BP. Große Siedlungsgebiete im Nahbereich und eine verbesserte Verkehrsanbindung bis 2020 werden den Erholungsdruck auf den schon jetzt stark besuchten BP verstärken. Das Management des BP benötigt daher Forschungsergebnisse über die Auswirkungen dieser städtebaulichen Entwicklungen auf die Untere Lobau und Entscheidungsgrundlagen wie auf den ansteigenden Besucherdruck reagiert werden soll.

Im Rahmen des vorliegenden Projektes wurde die Wirksamkeit verschiedener Maßnahmen innerhalb und außerhalb des BP, zur Entlastung der Oberen und Unteren Lobau hinsichtlich des zu erwartenden Besucherdrucks, untersucht. Besonderer Fokus lag dabei auf der Frage, inwieweit eine Ausgestaltung des Lobau-Vorlandes die Besuchsbelastung im BP reduzierten kann. Dazu wurden neue und miteinander kombinierte Forschungs- und Simulationsmethoden eingesetzt. Mehrere Planungs- und Managementszenarien wurden hinsichtlich der Aufnahmefähigkeit von Besuchermengen und Nutzergruppen getestet und verglichen.

Die Ziele des Projektes waren:

- Darlegung der Besuchsbelastung für die Lobau und den Biosphärenpark Untere Lobau in rund 15 Jahren,

- Aufzeigen von Lösungsmöglichkeiten zur Reduktion der Besuchsbelastung in der Lobau mit dem Schwerpunkt landschaftliche Ausgestaltung des Lobau-Vorlandes (Definition von Planungs- und Managementszenarien),

- Simulation von Planungs- und Managementszenarien hinsichtlich der Aufnahmefähigkeit von Besuchermengen im Lobau-Vorland zur Entlastung der Lobau,

- Bewertung der Planungs- und Managementszenarien hinsichtlich der Reduktion von Besuchermengen in der Lobau und

- Identifikation der nachhaltigsten Maßnahmen.

Als Basis zur Bemessung der Auswirkungen der Maßnahmenbündel v.a. in Hinblick auf die Besucherzahlreduktion in der Lobau wurde das Szenario „2025“ als „Laissez-faire -Szenario“ definiert. Dieses beschreibt eine Situation, wo keinerlei Maßnahmen getroffen werden und somit die prognostizierte Besucherzahlentwicklung, die eine Verdoppelung der Besuchszahlen gegenüber 1999 anzeigt, voll wirksam auf die Lobau wird.

Im Rahmen des Projektes wurden daher vier Basisszenarien definiert und simuliert, die aus Maßnahmen im Lobau-Vorland und in der Lobau bestanden. Die Maßnahmen im Lobau-Vorland unterteilten sich in eine lineare und flächige landschaftliche Ausgestaltung des Vorlandes. Unter der linearen Ausgestaltung ist die Anlage eines attraktiven Radwegenetzes tlw. auf bestehenden Straßen, die für den motorisierten Verkehr (AnwohnerInnen ausgenommen) zu sperren sind, zu verstehen. Dieses neue Radwegenetz soll v.a. ein Hineinfahren in die Lobau für die Radfahrenden weniger attraktiv machen. Einige Parkplätze werden von der Lobau weg verlegt, um den Besuchswiderstand zu erhöhen und die BesucherInnen zu zwingen, auch die neuen Wege des Vorlandes zu benutzen. Die flächige Ausgestaltung inkludiert das oben erwähnte Radwegenetz sowie das Parkraummanagement, eine Ausgestaltung des Lobau-Vorlandes mit einer Wald-Wiesen-Landschaft und ein attraktives Wanderwegenetz. Maßnahmen in der Lobau beinhalten entweder die derzeitige Gewässersituation oder eine Anbindung des Hauptgewässerzuges der Unteren Lobau an die Donau (Gewässerszenario).

Die Ergebnisse der agentenbasierten Simulationen zeigten, dass bei einer Attraktivierung des LobauVorlandes

- rund $30 \%$ weniger Besucherkilometer/Tag in der Lobau gegenüber dem „Laissez-faire Szenario“ (2025) zu verzeichnen sind, dies gilt sowohl für Werk- wie Spitzentage.

- dennoch eine 1,6-fache Erhöhung der Besuchsbelastung gegenüber dem Jahr 1999 gegeben ist. 
- die Pufferwirkung v.a. auf die Obere Lobau wirkt, die Untere Lobau hinsichtlich Besucherkilometer aber kaum entlastet wird.

Somit ist für die Untere Lobau eine Erhöhung der Besuchsbelastung gegenüber 1999 um das 2,4 bis 3,1-fache zu verzeichnen, jene der Oberen Lobau erhöht sich gegenüber 1999 um das 1,3 bis 1,5fache. Eine flächige Ausgestaltung des Lobau-Vorlandes mit einer Wald-Wiesen-Mosaiklandschaft kann die Besuchermenge in der Lobau nicht wesentlich mehr reduzieren als die lineare Ausgestaltung, ist aber aus stadtökologischer Sicht vorteilhaft. Das Gewässerszenario (Anbindung des Hauptgewässerzuges der Unteren Lobau an die Donau) hat nahezu keine Pufferwirkung im Hinblick auf die Besuchszahlen. Es ermöglicht allerdings eine starke Beruhigung von Bereichen der Unteren Lobau und bedeutet eine ökologische Aufwertung des Gebietes und eine langfristige positive Entwicklung vor allem der aquatischen und halb-aquatischen Lebensräume.

Trotz der vorgeschlagenen Maßnahmen wird die Lobau aufgrund der städtebaulichen Entwicklung noch mehr unter Druck kommen. Vor allem die Besucherzunahme an Werktagen ist kritisch, da diese eine hohe Eintrittshäufigkeit aufweisen. Problematisch ist das besonders für die Obere Lobau, da diese schon jetzt teilweise über ihre Tragfähigkeitsgrenze belastet ist (Arnberger et al., 2009). Die starke Erhöhung der Besuchsbelastung des bisher weniger intensiv genutzten BP Untere Lobau ist ebenfalls kritisch zu sehen. Neben der Zunahme an Besuchen war auch ein Ausweichverhalten von der Oberen in die Untere Lobau aufgrund der höheren Besuchermengen in der Oberen Lobau festzustellen. Dies könnte in der Unteren Lobau künftig ebenfalls zu einer Überschreitung der Tragfähigkeitsgrenzen an den Wochenenden führen. Maßnahmenbündel insbesondere für den 22. Wiener Gemeindebezirk sowie für die Gemeinde Groß-Enzersdorf sind daher in weiterer Folge zu definieren und zu simulieren. 


\section{Einleitung}

\subsection{Problemstellung}

Der Wiener UNESCO Biosphärenpark (BP) Untere Lobau ist ein durch Grundwasser und Rückstau gespeister Auwald und beherbergt ein äußerst vielfältiges Mosaik an aquatischen, halb-aquatischen und terrestrischen Habitaten. Gleichzeitig leben rund 150.000 Menschen in direkter Nachbarschaft zum BP. Große Siedlungsgebiete im Nahbereich und eine verbesserte Verkehrsanbindung bis 2025 werden den Erholungsdruck auf den schon jetzt stark besuchten BP verstärken. Das Management des BP benötigt daher Forschungsergebnisse über die Auswirkungen dieser städtebaulichen Entwicklungen auf den BP und Entscheidungsgrundlagen wie sie mit dem ansteigenden Besucherdruck umgehen kann.

\subsection{Projektziele}

Im Rahmen des Projektes wurde die Wirksamkeit verschiedener Maßnahmen innerhalb und außerhalb des BP zur Entlastung der Oberen und Unteren Lobau hinsichtlich des zu erwartenden Besucherdrucks untersucht. Planungs- und Managementszenarien wurden hinsichtlich der Aufnahmefähigkeit von Besuchermengen/Nutzergruppen gemeinsam mit Stakeholdern entwickelt, simuliert und verglichen. Die Ziele des Projektes waren:

- Darlegung der Besuchssituation für die Lobau in rund 15 Jahren

- Definition von Planungs- und Managementszenarien

- Test der entwickelten Szenarien hinsichtlich der Aufnahmefähigkeit von Besuchermengen im Lobau-Vorland zur Entlastung der Lobau

Folgende Prinzipien bei der Szenariendefinition wurden dabei angestrebt:

- Sicherung/Steigerung der ökologischen Funktionsfähigkeit der Lobau

- Sicherung/Steigerung der Erholungsqualität

- $\quad$ Stadt Wien als attraktiver Wohnort

- Konfliktvermeidung durch Ausgleichsmaßnahmen und Ersatzflächen

- Indirekte Besucherlenkung prioritär gegenüber direkter Besucherlenkung

- Nachhaltige Kombination von Maßnahmen in der Lobau und im Lobau-Vorland

\subsection{Projektablauf und Untersuchungsraum}

Der Ablauf des Projektes gliedert sich grob in fünf Arbeitsschritte (Abb. 1). Im ersten Schritt wurde die Anzahl künftiger Lobaubesuche aufgrund der Stadtentwicklung im Nahbereich der Lobau abgeschätzt. Dann wurden gemeinsam mit Stakeholdern mögliche Planungs- und Managementszenarien definiert. Die entwickelten Szenarien wurden anschließend hinsichtlich der Aufnahmefähigkeit von Besuchermengen im Lobau-Vorland zur Entlastung der Lobau simuliert. Die Ergebnisse der Simulationen als auch die Maßnahmen an sich wurden anschließend bewertet und Vorschläge für die Planung und das Management des Biosphärenparks und sein Umfeld unter Einbezug von Stakeholdern ausgearbeitet. 
Definition der Ausgangslage Lobau 2025 (Besuchermengenprognose)

Definition von möglichen Maßnahmen unter Einbezug von Stakeholdern

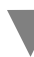

Simulation der Maßnahmen hinsichtlich der Reduktion des Besucherdrucks auf die Lobau inkl. Biosphärenpark Untere Lobau

Bewertung der Simulationen anhand von Kriterien durch Fachdisziplinen

Auswahl der nachhaltigsten Maßnahmen unter Einbezug von Stakeholdern und

Ableitung von Empfehlungen für das Management des Biosphärenparks

Abb. 1: Arbeitsschritte des Projektes

Der Untersuchungsraum umfasst drei räumliche Einheiten (Abb. 2):

- Die Lobau mit der Oberen Lobau und dem UNESCO Biosphärenpark Untere Lobau.

- Das Lobau-Vorland inkl. der angrenzenden Siedlungsbereiche Wien 22 und Groß-Enzersdorf.

- Die angrenzenden Stadtentwicklungsbereiche in Wien 22 mit Schwerpunkt Seestadt Aspern.

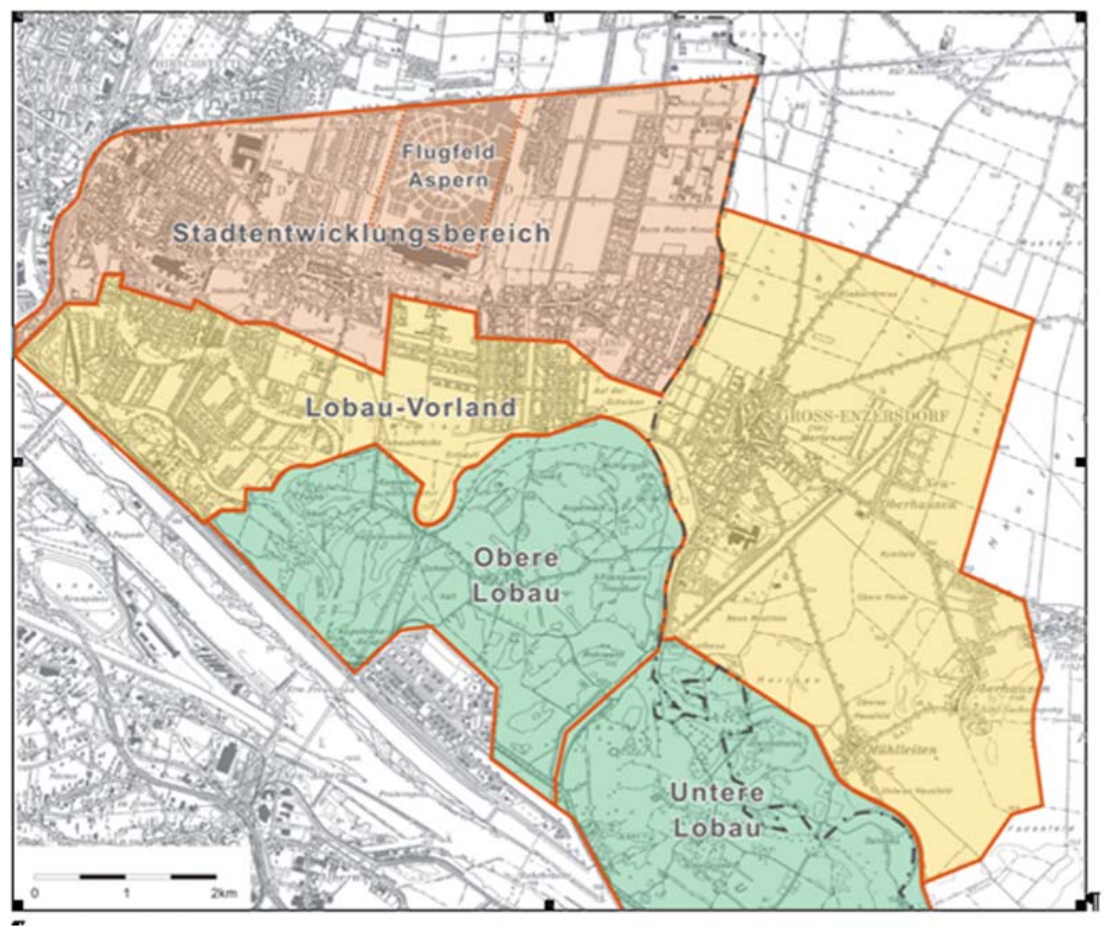

Abb. 2: Bearbeitungsgebiet Projekt Lobau 2020 


\section{Prognose des Besucherdrucks auf die Lobau im Jahr 2025 aufgrund städtebaulicher Entwicklungen}

\subsection{Grundlagen für die Prognose der Besuchshäufigkeiten}

Eine Grundlage der Prognose für den Zuwachs an Besuchen in die Lobau für das Jahr 2025 bildete die Siedlungsentwicklung im 22. Wiener Gemeindebezirk und in der Gde. Groß-Enzersdorf. Diese Informationen wurden über Gespräche mit den jeweiligen Planungsverantwortlichen erhoben und zusammengeführt.

Die zweite Grundlage waren die Ergebnisse einer Befragung, die 2006 an die Bewohner und Bewohnerinnen des 22. Bezirkes und jene der Gde. Groß-Enzersdorf gerichtet war (Arnberger et al., 2009). Diese postalische Befragung lief nach dem für postalische Befragungen üblichem Schema (Dillman, 1983) ab:

- Ankündigungsschreiben (erhielt jeder 2. zufällig ausgewählte Haushalt)

- $\quad$ 1. Fragebogenzusendung (Anfang Februar 2006)

- 1. Erinnerungsschreiben (Reminder in Form einer Postkarte) - Aussendung (Februar 2006)

- 2. Fragebogenzusendung (Ende Februar/Anfang März 2006)

- 2. Erinnerungsschreiben in Form einer Postkarte (erhielten Mitte März 2006 jene Haushalte, die kein Ankündigungsschreiben erhalten hatten)

Insgesamt 1216 BewohnerInnen wurden aus dem Einwohnerregister nach dem Zufallsprinzip für die Postbefragung ausgewählt. 56 Fragebögen kamen als nicht zustellbar zurück. Von den 1160 zugestellten Fragebögen wurden 611 per Nachnahme zurückgeschickt, davon waren 602 brauchbar. Die Rücklaufquote betrug somit $53 \%$ und kann als recht zufrieden stellend angesehen werden. Nahezu die Hälfte der Fragebögen kam aus der Gde. Groß-Enzersdorf. Drei Viertel der Antwortenden reagierten bereits auf die erste Fragebogenaussendung.

Ergebnis der Befragung war, dass $98 \%$ der Befragten die Lobau schon einmal besucht hatten. Die berichteten Besuchshäufigkeiten von jenen, die die Lobau besuchten, schwankten während des Jahres vor der Befragung zwischen einem und 2000 Besuchen, der Mittelwert lag bei 65 Besuchen, der Median bei 25 Besuchen. Allerdings unterschieden sich die Besuchshäufigkeiten zwischen den im 22. Bzk. Wohnenden zu jenen aus der Gde. Groß-Enzersdorf deutlich. Während der Mittelwert bei der ersten Gruppe bei 37,8 Besuchen im Jahr (Median 15 Besuche) lag, zeigten die Groß-Enzersdorfer eine deutlich höhere Besuchshäufigkeit mit 87,5 Besuchen im Durchschnitt (Median 40 Besuche). Berücksichtigt man die Einwohnerzahlen (22. Bzk., Gde. GE) so liegt der gewichtete Mittelwert bei ca. 40 durchschnittlichen Besuchen im Jahr.

Werden die Besuchshäufigkeiten unter Einbeziehung auch jener, die die Lobau bisher noch nicht besucht haben, berechnet, ergibt sich ein Mittelwert der im 22. Bzk. Wohnenden von 31,5 Besuchen im Jahr (Median 10 Besuche). Die Groß-Enzersdorfer hatten eine Besuchshäufigkeit mit 84,3 Besuchen im Durchschnitt (Median 40 Besuche).

\subsection{Einwohner- und Arbeitsplatzprognose}

\subsubsection{Vorgangsweise}

Die entscheidende Determinante für die Aufgabenstellung der Untersuchung ist die künftige Entwicklung der Anzahl der EinwohnerInnen und Arbeitsplätzen im Untersuchungsraum. Sie bestimmt im Wesentlichen nicht nur die Verkehrsnachfrage insgesamt, sondern auch die Verkehrsnachfrage im Freizeitverkehr, woraus der Bedarf an Erholungsflächen abgeleitet werden kann. Zur Fragestellung liegen im ÖIR Prognoseergebnisse vor, die im Rahmen der Netzanalyse der Wiener Linien auf Basis des Jahres 2005 erstellt wurden. Für den städtebaulichen Schwerpunkt, dem Stadtentwicklungsprojekt Flugfeld Aspern, wurde die Prognose nach Angaben des Masterplans ergänzt. 


\subsubsection{Masterplan ehemaliges Flugfeld Aspern („Seestadt Aspern“)}

Die im Februar 2006 gestartete Masterplanung Flugfeld Aspern legt die städtebauliche Entwicklung des mit 240 Hektar größten Stadtentwicklungsgebietes von Wien fest (Abb. 3). Geplant ist ein multifunktioneller Stadtteil, nun Seestadt Aspern genannt, mit den folgenden Schwerpunkten:

- Wohnen

- Verwaltungseinrichtungen, Büros, Dienstleistungen, Handel und Geschäfte

- Wissenschafts- und Bildungseinrichtungen mit überregionaler Bedeutung für Forschung \& Entwicklung sowie universitäre Ausbildung

- Gewerbe und Produktion

- $\quad$ Einrichtungen für Soziales, Freizeit, Sport, Erholung und Kultur

- großzügige Grünflächen (u.a. ein Park mit großzügiger Wasserfläche)

In der Endausbaustufe sind bis zu 18.000 Büro- und Dienstleistungsarbeitsplätze, 7.000 Arbeitsplätze in Produktion und Gewerbe sowie rund 8.500 Wohnungen vorgesehen. Die räumlich-funktionale Schwerpunktsetzung sieht folgendermaßen aus:

- Wohnen vor allem im Westen und Südwesten, teilweise auch im Norden und im Kerngebiet

- kommerzielles Zentrum mit Verwaltungseinrichtungen, Büros, Dienstleistungen, Handel und Geschäften im Norden rund um den Bahnhof (Knotenpunkt Öffentlicher Verkehr) und den Hauptplatz

- Universität/ Forschung \& Entwicklung im Süden und Südosten

- Schulen im Westen und Süden (1 AHS, 2 Hauptschulen, 2 Volksschulen, 1 Sonderschule)

- Gewerbe- und Produktionsflächen an den südlichen und östlichen Randlagen

- Park mit großzügiger Wasserfläche in der Mitte des Flugfelds für Sport-, Kultur- und Freizeitfunktionen

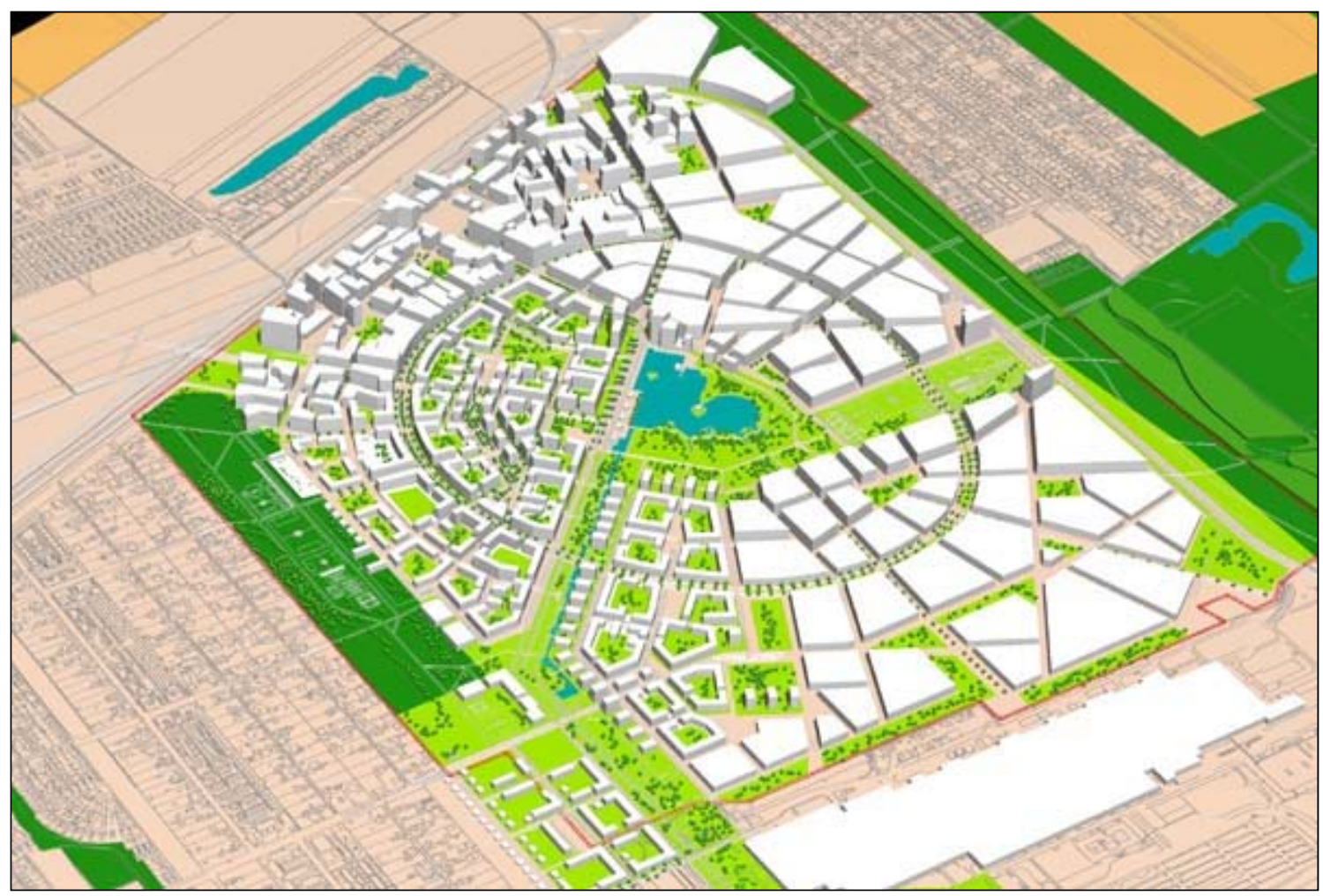

Abb. 3: 3D-Darstellung der geplanten Bebauung am Flugfeld Aspern. Quelle: Masterplan Flugfeld Aspern 
Tab. 1 gibt einen Überblick über die geplanten Entwicklungen am Flugfeld Aspern basierend auf den einzelnen Entwicklungsetappen.

\begin{tabular}{|l|l|l|l|}
\hline & $\begin{array}{c}\text { Fertig- } \\
\text { stellung }\end{array}$ & $\begin{array}{c}\text { Rahmenbedingungen vor } \\
\text { der Etappe }\end{array}$ & \multicolumn{1}{c|}{ Bebauung } \\
\hline Phase 0 & $2007-10$ & Neue Straße zur B3 Südost & $\begin{array}{l}\text { Im Osten bei Südost-Straße: Gewerbe, } \\
\text { Studentenwohnheim, Einrichtungen der Universität }\end{array}$ \\
\hline Phase 1 & $2010-13$ & $\begin{array}{l}\text { U2 bis Aspernstraße in Be- } \\
\text { trieb, U2 im Flugfeld in Bau; } \\
\text { Baubeginn A23 }\end{array}$ & $\begin{array}{l}\text {,gallisches Dorf" im Südwesten und entlang Ring bis } \\
\text { Gewerbegebiet im Südosten: vor allem Wohnen 1.000- } \\
1.500 \text { Wohneinheiten mit Infrastruktur (Schule) }\end{array}$ \\
\hline Phase 2 & $2013-19$ & $\begin{array}{l}\text { U2 bis Flugfeld in Betrieb, } \\
\text { A23 in Betrieb }\end{array}$ & $\begin{array}{l}\text { Hauptentwicklungsphase: ganze Südhälfte, Beginn der } \\
\text { Entwicklung im dichten Kerngebiet (U-Bahn-Stationen, } \\
\text { Hauptstraße) }\end{array}$ \\
\hline Phase 3 & ab 2020 & \multicolumn{2}{|l}{$\begin{array}{l}\text { Nachverdichtung, Fertigstellung Kerngebiet, } \\
\text { Gewerbegebiet im Nordosten }\end{array}$} \\
\hline
\end{tabular}

Tab. 1: Entwicklungsetappen Masterplan Flugfeld Aspern. Quelle: Projektteam Flugfeld Aspern

Aus den Erfordernissen der Netzentwicklungen wurden für die Untersuchung zwei Zeithorizonte definiert:

- 2015: Fertigstellung der U2-Nord bis zur Station Seestadt; Inbetriebnahme des Hauptbahnhof Wien;

- 2025: Eisenbahnschnellverkehr Wien - Bratislava sowie Regionalexpresszüge mit Halt in der neu zu errichtenden ÖBB-Station Aspern (vormals Flugfeld Nord).

Da die für die Untersuchung maßgeblichen Zeithorizonte 2015 bzw. 2025 nicht zur Gänze mit den Entwicklungsetappen im Masterplan Flugfeld Aspern übereinstimmten, wurden die Einwohner- und Arbeitsplatzzahlen gemäß den vier Entwicklungsetappen des Masterplans den Zeithorizonten 2015 und 2025 zugeordnet.

Für den Zeithorizont 2015 wurden die Phasen 0 und 1 zur Gänze berücksichtigt, die Phase 2 zur Hälfte. Für den Zeithorizont 2025 wurden die Phasen 0 bis 2 vollständig berücksichtigt, die Phase $3 \mathrm{zu}$ $50 \%$ bei den EinwohnerInnen bzw. zu $30 \%$ bei den Arbeitsplätzen. Die Zuordnung der Entwicklungsetappen zu den Zeithorizonten zeigt Tab. 2.

\begin{tabular}{|c|c|c|c|c|}
\hline Zeithorizont & $\begin{array}{c}\text { Phase 0 } \\
(2007-10)\end{array}$ & $\begin{array}{c}\text { Phase } 1 \\
(2010-13)\end{array}$ & $\begin{array}{c}\text { Phase } 2 \\
(2013-19)\end{array}$ & $\begin{array}{c}\text { Phase } 3 \\
\text { (ab 2020) }\end{array}$ \\
\hline 2015 & $100 \%$ & $100 \%$ & $\begin{array}{l}\text { EW } 50 \% \\
\text { APL } 50 \%\end{array}$ & $0 \%$ \\
\hline 2025 & $100 \%$ & $100 \%$ & $100 \%$ & $\begin{array}{l}\text { EW } 50 \% \text {, } \\
\text { APL } 30 \%\end{array}$ \\
\hline
\end{tabular}

Tab. 2: Zuordnungsprinzip Entwicklungsetappen - Zeithorizonte Netzmodell

Im Vergleich zum Vollausbau des Flugfelds Aspern wird somit bis 2025 mit rund $80 \%$ der EinwohnerInnen und $60 \%$ der Arbeitsplätze bezogen auf den Vollausbau gerechnet.

\subsubsection{Einwohnerprognose übrige Bereiche}

Wie bereits in anderen Untersuchungen gezeigt wurde (Netzanalyse Wiener Linien, ÖIR), wird die anwesende Bevölkerung Wiens als Grundlage der Einwohner- und der Verkehrsprognose herangezogen, weil dadurch die an einem durchschnittlichen Werktag - wenn Schultag - anwesende Bevölkerung erfasst wird. Mit der Wohnbevölkerung (Hauptwohnsitz) kann die werktägliche Verkehrsnachfrage nur unzureichend erfasst werden (tendenziell Untererfassung am Stadtwohnsitz; dies betrifft insbesondere auch die in Wien Studierenden). Die Einwohnerprognose erfolgt somit unter Hinzurechnung der Bevölkerung am Nebenwohnsitz. 
Die Grundlagen für die Einwohnerprognose waren:

- Bevölkerungsfortschreibung für Wien vom 31.3.2004 und 31.12.2005 nach Zählgebieten

- Planungen und Projekte, die dem Stadtentwicklungsplan Wien 2005 (STEP 05) zugrunde liegen

- die im Rahmen der Bearbeitungen zum Masterplan Flugfeld Aspern übernommenen Annahmen

- Berücksichtigung aktueller Entwicklungen (u.a. aufgrund ergänzender Gespräche mit der MA 18 und MA 21)

- Bevölkerungsvorausschätzung 2006-2050 der Statistik Austria für Wien, 2006

Die Ergebnisse der Einwohnerprognose 2004-2025 (Tab. 3) zeigen:

- einen deutlichen Anstieg der Einwohnerzahl Wiens

- starke Zuwächse im 22. Bezirk (Donaustadt wird mit über 200.000 EinwohnerInnen zum bevölkerungsreichsten Bezirk Wiens)

- eine nahezu Verdoppelung der EinwohnerInnen im Untersuchungsraum, wobei die 16.400 EinwohnerInnen im Flugfeld Aspern wesentlich dazu beitragen (weitere Projekte befinden sich in Aspern an der U2, im Hausfeld, am Lackenjöchl und Heidjöchl sowie in Eßling)

- Wohnbebauung im Flugfeld bis 2015:

- Wohnen vor allem im Südwesten mit 1.000 - 1.500 Wohnungen und sozialer Infrastruktur (z.B. Schule) sowie teilweise bereits im westlichen Bereich

- Studentenwohnheim im Süden

- Wohnbebauung im Flugfeld 2015 bis 2025:

- weitere Verdichtungen im Südwesten und Westen

- Wohnen auch im Norden und im Kerngebiet

\begin{tabular}{|l|r|r|r|r|}
\hline & \multicolumn{1}{|c|}{$\mathbf{2 0 0 4}$} & \multicolumn{1}{c|}{$\mathbf{2 0 1 5}$} & \multicolumn{1}{c|}{$\mathbf{2 0 2 5}$} & \multicolumn{1}{c|}{$\mathbf{2 0 0 4 - 2 5}$} \\
\hline Wien insgesamt & 1.627 .400 & 1.747 .500 & 1.819 .200 & +191.800 \\
\hline 22. Bezirk & 143.700 & 190.700 & 205.600 & +61.900 \\
\hline Flugfeld Aspern (nunmehr Seestadt & 0 & 9.700 & 16.400 & +16.400 \\
Aspern) & 40.000 & 59.000 & 63.000 & +23.000 \\
\hline Einzugsbereich & 40.000 & 68.700 & 79.400 & +39.400 \\
\hline Untersuchungsraum & & &
\end{tabular}

Tab. 3: Einwohnerprognose 2004-2025. Quelle: ÖIR, Verkehrsmodell Wien.

\subsubsection{Arbeitsplatzprognose übrige Bereiche}

Grundlage für die Arbeitsplatzprognose waren:

- die Arbeitsstättenzählung 2001 der Statistik Austria (nach Zählgebieten und ÖNACEAbschnitten);

- Planungen und Projekte, die dem Stadtentwicklungsplan Wien 2005 (STEP 05) zugrunde liegen;

- die im Rahmen der Bearbeitungen zum Masterplan Flugfeld Aspern übernommenen Annahmen;

- die Berücksichtigung aktueller Entwicklungen durch das ÖIR (u.a. aufgrund ergänzender Gespräche mit der MA 18 und MA 21);

- die Beschäftigungsprognose für Österreich 2000-2008 des WIFO, die aufgrund der unterschiedlichen Bedingungen für Wien vom ÖIR modifiziert wurde (z.B. stärkere Entwicklung des Dienstleistungssektors).

Die Ergebnisse der Arbeitsplatzprognose 2004-2025 (Tab. 4) zeigen:

- einen mäßigen, aber kontinuierlichen Anstieg der Arbeitsplätze in Wien;

- $\quad$ einen starken Anstieg im 22. Bezirk;

- eine Verdreifachung der Arbeitsplätze im Untersuchungsraum, die zum Großteil auf die über 16.000 neuen Arbeitsplätze am Flugfeld zurückzuführen ist;

- eine geringe Zunahme im weiteren Einzugsbereich; 
- Arbeitsplatzentwicklung im Flugfeld bis 2015:

- universitäre Einrichtungen sowie Forschung \& Entwicklung im Südosten (aus heutiger Sicht unsicher)

- Gewerbe und Produktion an den südlichen und östlichen Randlagen, Versorgungsund Dienstleistungsangebot an den U-Bahn Stationen

- Arbeitsplatzentwicklung im Flugfeld 2015 bis 2025:

- Verwaltungseinrichtungen, Büros, Dienstleistungen, Handel und Geschäfte im Norden rund um den Bahnhof (kommerzielles Zentrum)

- Gewerbe und Produktion an den Randlagen im Nordosten

\begin{tabular}{|l|r|r|r|r|}
\hline & \multicolumn{1}{|c|}{$\mathbf{2 0 0 4}$} & \multicolumn{1}{c|}{$\mathbf{2 0 1 5}$} & \multicolumn{1}{c|}{$\mathbf{2 0 2 5}$} & \multicolumn{1}{c|}{$\mathbf{2 0 0 4 - 2 5}$} \\
\hline Wien insgesamt & 831.700 & 869.900 & 900.000 & +68.300 \\
\hline 22. Bezirk & 48.900 & 63.600 & 74.500 & +25.600 \\
\hline Flugfeld Aspern (nunmehr Seestadt & 0 & 6.300 & 16.100 & +16.100 \\
Aspern) & 6.800 & 7.900 & 8.400 & +1.600 \\
\hline Einzugsbereich & 6.800 & 14.200 & 24.500 & +17.700 \\
\hline Untersuchungsraum & & &
\end{tabular}

Tab. 4: Arbeitsplatzprognose. Quelle: ÖIR, Verkehrsmodell Wien.

Abbildung Strukturdaten im und um das Flugfeld Aspern. Quelle: ÖIR, 2006. Sekundärnetz Flugfeld Aspern.Abb. 4 gibt eine Übersicht über die Verteilung von EinwohnerInnen, Arbeitsplätzen und Schulplätzen im und um das Flugfeld Aspern.

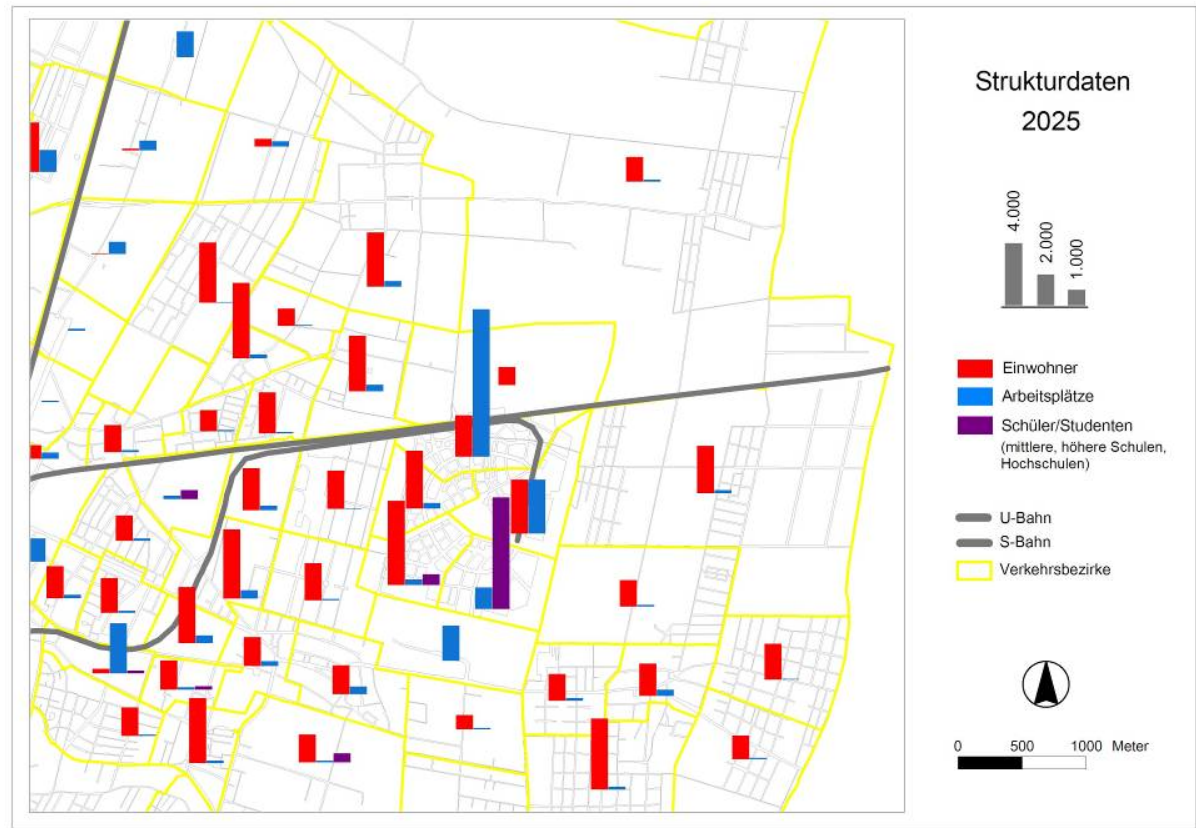

Abb. 4: Strukturdaten im und um das Flugfeld Aspern. Quelle: ÖIR, 2006. Sekundärnetz Flugfeld Aspern.

\subsubsection{Prognose übriges Wien}

Für die übrigen Bereiche Wiens wurde wie folgt vorgegangen. Grundlagen für die Einwohnerprognose für Wien 2015 und 2025 sind:

- als Ausgangsbasis die Bevölkerungsevidenz 31.3.2004 der Stadt Wien (nach Zählgebieten und Altersklassen);

- Planungen und Projekte, die dem Stadtentwicklungsplan 2005 (STEP 2005) der Wiener Stadtplanung (MA 18) zugrunde liegen;

- die Altersklassenanteile aus der Bevölkerungsvorausschätzung von Statistik Austria (2006), Hauptszenario 2006-2050. 
Darauf aufbauend wurden folgende Prognoseschritte gesetzt:

- Annahmen bezüglich Lückenfüllungen und Verdichtungen

- Berücksichtigung von spezifischen Strukturentwicklungsfaktoren (Abwanderungsfaktor, Bezirksgruppenfaktor, Siedlungstypenfaktor)

- $\quad$ ergänzende Gespräche mit der MA 21 (Stadtteilplanung)

- $\quad$ sowie aktuelle Informationen zu den Projekten in Medien und Internet.

Die Arbeitsplatzprognose für Wien 2015 sowie 2025 basiert auf folgenden Grundlagen:

- als Ausgangsbasis die Arbeitsstättenzählung 2001 (nach Zählgebieten und ÖNACEAbschnitten)

- die Entwicklung der Beschäftigtenstruktur (NACE-2) adaptiert nach Quellen des Wirtschaftsforschungsinstituts (Beschäftigungsprognose für Oberösterreich 2000-2008, 2002)

- Planungen und Projekte, die dem Stadtentwicklungsplan 2005 (STEP 2005) der Wiener Stadtplanung (MA 18) zugrunde liegen.

Weiters waren folgende Prognoseschritte notwendig:

- Hochrechnung der Arbeitsplätze nach Wirtschaftsabteilungen

- ergänzende Gespräche mit der MA 21 (Stadtteilplanung)

- $\quad$ sowie aktuelle Informationen zu den Projekten in Medien und Internet.

\subsubsection{Prognose Groß-Enzersdorf}

Für Groß-Enzersdorf (Bestand 2007: 9.000 EinwohnerInnen) werden nach einem Gespräch mit der Gemeinde (Bürgermeister und Planungsstadtrat) auf Grund der Kenntnis bestehender Siedlungsprojekte, des vorhandenen Baulands und der Impulse aus der Realisierung der S1 Lobauquerung bis 2020 rund 15.000 EinwohnerInnen erwartet. Die Werte für 2015 wurden interpoliert, die Abschätzung der weiteren Entwicklung bis 2025 geht von einer leicht abgeschwächten weiteren Siedlungsentwicklung aus.

\subsubsection{Zusammenfassung}

Aus den Planungen zum Flugfeld Aspern und den Planungen für Wien und Umland kann folgende Einteilung des Untersuchungsraumes abgeleitet werden:

- Lobau (Obere und Untere Lobau)

- Lobau-Vorland (keine signifikanten Stadterweiterungsvorhaben geplant). Die Gemeinde Groß-Enzersdorf wird auf Grund ihrer Nähe, mit Ausnahme der Lobau-fernen Teile, dem Lobau-Vorland zugerechnet.

- Stadtentwicklungsbereich

Im Jahr 2007 lebten rund 61.000 EinwohnerInnen im Einzugsbereich der Lobau. Nach den Prognosen werden die Einwohnerzahlen künftig kräftig zunehmen, bis 2015 auf rund 90.000 und bis 2025 auf rund 103.000 (Tab. 5). Dies bedeutet einen Zuwachs im 22. Bezirk von ca. 47.100 EinwohnerInnen, quasi die Errichtung einer Stadt, die nicht wesentlich unter der Größe St. Pölten liegt. Rund 42.000 EinwohnerInnen werden direkt im Lobau-Einzugsbereich wohnen. Für die Seestadt Aspern ist in der ersten Phase aus heutiger Sicht eine langsame Entwicklung wahrscheinlich, entsprechend wurden die Werte für 2015 nach unten korrigiert. Die Werte für 2025 wurden beibehalten; mit einer Vollerschließung lägen sie rund weitere 15.000 EinwohnerInnen höher (langfristige Perspektive). $\mathrm{Zu}$ beachten ist auch, dass im Lobau-Vorland eine nicht unbeträchtliche Dynamik um zusätzliche 10.000 EinwohnerInnen zu erwarten ist. 


\begin{tabular}{|c|c|c|c|c|c|}
\hline \multirow[b]{2}{*}{ EZB Wien } & \multicolumn{3}{|c|}{ EinwohnerInnen } & \multicolumn{2}{|c|}{ Entwicklung abs. } \\
\hline & 2007 & 2015 & 2025 & $2007-15$ & 2007-25 \\
\hline Lobau & 20 & 20 & 20 & 0 & 0 \\
\hline Lobau-Vorland Wien & 16.000 & 18.400 & 19.900 & 2.400 & 3.900 \\
\hline Stadtentwicklungsbereich & 37.700 & 60.100 & 70.000 & 21.400 & 32.300 \\
\hline davon Seestadt Aspern & 0 & 6.200 & 16.400 & 6.200 & 16.400 \\
\hline 22. Bezirk Rest & 104.800 & 109.700 & 115.700 & 4.900 & 10.900 \\
\hline Wien Rest & 1.522 .800 & 1.562 .500 & 1.613 .600 & 39.500 & 90.800 \\
\hline Wien insgesamt & 1.681 .300 & 1.749 .500 & 1.819 .200 & 68.200 & 137.900 \\
\hline Groß-Enzersdorf, Anteil Lobau-Vorland & 7.000 & 9.300 & 13.100 & 2.300 & 6.100 \\
\hline übriges Groß-Enzersdorf & 2.100 & 2.700 & 3.900 & 600 & 1.800 \\
\hline Groß-Enzersdorf insgesamt & 9.100 & 12.000 & 17.000 & 2.900 & 7.900 \\
\hline Lobau und Lobau-Vorland (inkl. Gr.-Enz.) & 23.000 & 27.700 & 33.000 & 4.700 & 10.000 \\
\hline Stadtentwicklungsbereich & 37.700 & 59.100 & 70.000 & 21.400 & 32.300 \\
\hline Einzugsbereich Lobau insgesamt & 60.700 & 86.800 & 103.000 & 26.100 & 42.300 \\
\hline
\end{tabular}

Tab. 5: Einwohnerprognose. Quelle: ÖIR auf Grundlage Netzanalyse Wiener Linien 2007 sowie eigener Erhebungen

Die Arbeitsplatzprognose (Tab. 6) zeigt ein ähnliches Bild wie die Einwohnerprognose:

\begin{tabular}{|l|r|r|r|r|r|}
\hline \multicolumn{1}{|c}{ EZB Wien } & \multicolumn{3}{c|}{ Arbeitsplätze } & \multicolumn{2}{c|}{ Entwicklung abs. } \\
\cline { 2 - 6 } \multicolumn{1}{|c|}{} & $\mathbf{2 0 0 7}$ & $\mathbf{2 0 1 5}$ & $\mathbf{2 0 2 5}$ & $\mathbf{2 0 0 7}-\mathbf{1 5}$ & \multicolumn{1}{c|}{$\mathbf{2 0 0 7 - 2 5}$} \\
\hline Lobau & 410 & 360 & 330 & -50 & -80 \\
Lobau-Vorland Wien & 1.100 & 1.100 & 1.170 & 0 & 70 \\
Stadtentwicklungsbereich & 12.200 & 17.200 & 30.300 & 5.000 & 18.100 \\
davon Seestadt Aspern & 0 & 3.300 & 16.100 & 3.300 & 16.100 \\
22. Bezirk Rest & 42.100 & 42.000 & 42.800 & -100 & 700 \\
Wien Rest & 786.400 & 809.300 & 825.500 & 22.900 & 39.100 \\
\hline Wien insgesamt & 842.200 & 870.000 & 900.100 & 27.800 & 57.900 \\
\hline \multicolumn{7}{|l|}{} \\
\hline Lobau und Lobau-Vorland* & 1.500 & 1.500 & 1.500 & -100 & 0 \\
Stadtentwicklungsbereich* & 12.200 & 17.200 & 30.300 & 5.000 & 18.100 \\
\hline Einzugsbereich Lobau insgesamt* & 13.700 & 18.700 & 31.800 & 4.900 & 18.100 \\
\hline
\end{tabular}

Tab. 6: Arbeitsplatzprognose. * Angaben ohne Groß-Enzersdorf

Für die Arbeitsplätze wird mit der verbesserten Erreichbarkeit (A23, U2), den Planungen (Seestadt Aspern) und dem Nachholbedarf (aktuell massives strukturelles Arbeitsplatzdefizit im Einzugsbereich) mit einer noch stärkeren Dynamik gerechnet. Dies betrifft zwar ausschließlich das Stadterweiterungsgebiet und nicht das Lobau-Vorland, wird aber ebenso höhere Besucherfrequenzen in der Lobau zur Folge haben. Bis 2015 wird im Wiener Einzugsbereich der Lobau mit einer Zunahme von rund 14.000 auf rund 19.000 Arbeitsplätze gerechnet, und bis 2025 mit einer verstärkten Dynamik auf insgesamt rund 32.000 Arbeitsplätze.

\subsection{Prognostizierter Besucherdruck auf die Lobau im Jahr 2025}

Basierend auf den Siedlungsentwicklungsplanungen und den Besuchshäufigkeiten der EinwohnerInnen wurden die Besuchszahlen für die Lobau für das Jahr 2025 prognostiziert. Dazu wurde nicht der Mittelwert der Besuchshäufigkeit pro EinwohnerIn, sondern der konservativere Median herangezogen (Tab. 7). Angenommen wurden für den 22. Bezirk ca. 47.100 neue EinwohnerInnen und für die Gemeinde Groß-Enzersdorf ca. 7.900 neue EinwohnerInnen. 
Damit ergibt sich eine prognostizierte Zunahme von rund 790.000 Besuchen im Jahr. Dies wäre mehr als eine Verdopplung der Besuchszahlen des Jahres 1998-1999 mit rund 600.000 Besuchen (Arnberger et al., 2000). Aus der Gemeinde Groß-Enzersdorf würde aufgrund der festgestellten sehr hohen Besuchsintensitäten $40 \%$ des Zuwachses stammen.

\begin{tabular}{|l|r|r|r|}
\hline \multicolumn{1}{|c|}{ Bereiche } & Neue EinwohnerInnen & $\begin{array}{c}\text { Besuchshäufigkeiten } \\
\text { (Median/pro Jahr) }\end{array}$ & $\begin{array}{c}\Sigma \\
\text { ¿ Besuche } \\
\text { pro Jahr }\end{array}$ \\
\hline Groß-Enzersdorf & 7.900 & 40 & 316.000 \\
\hline Wien XX11 & 47.100 & 10 & 471.000 \\
\hline Summe 2025 & 55.000 & & 787.000 \\
\hline
\end{tabular}

Tab. 7: Prognose der Besuchsintensitäten für die Lobau im Jahr 2025. 


\section{Szenarienentwicklung (Maßnahmenbündel) unter Einbezug von Stakeholdern}

\subsection{Grundlagen für die Szenariendefinition}

Nach der Prognose der Besucherzahlen für den Zeitraum um 2025 wurden Szenarien definiert, die die realistisch zu erwartenden Besucherströme reduzieren sollten. Damit verbunden sollte auch eine Aufwertung des Ökosystems Au aus gewässerökologischer Sicht sein.

\subsubsection{Maßnahmen aus gewässerökologischer Sicht in der Lobau}

Um eine ökologische Aufwertung der Lobau zu erreichen, wurde auf eine mögliche zukünftige Ausprägungsform der Unteren Lobau, die im Projekt Optima Lobau entwickelt wurde, zurückgegriffen. Das Szenario „Offen1b verlandet" basiert auf einer Anbindung des Hauptgewässerzuges der Unteren Lobau an die Donau, die zu einer deutlich erhöhten Gewässerdynamik und zur Förderung von auentypischen Standorten führt (Abb. 5). Die Anbindung resultiert neben einer veränderten Wasserführung (mehr Wasserflächen, durchströmte Bereiche) in einer geänderten Wasser- und Ufervegetation, sowie einer Intensivierung von Auf- und Abbauprozessen in den Gewässern. Das Wegenetz in der Unteren Lobau reduziert sich um ca. 4 km und einige der Wege werden als Stichwege geführt.

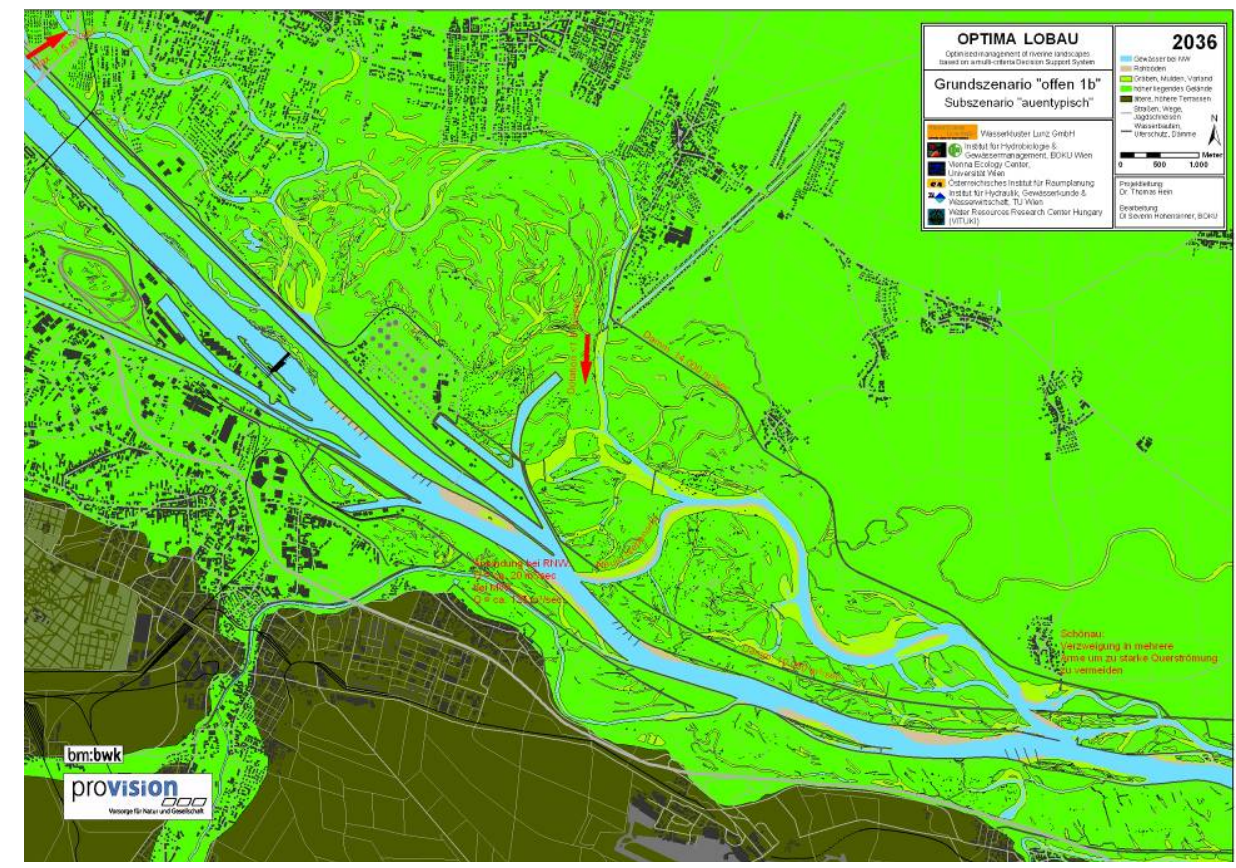

Abb. 5: Darstellung von Grundszenario „Offen 1b verlandet“ aus dem Projekt Optima Lobau.

\subsubsection{Maßnahmen aus erholungsplanerischer Sicht im Lobau-Vorland}

Die Maßnahmen der Erholungsplanung fokussierten auf das Lobau-Vorland. Ziel war, durch eine landschaftliche Ausgestaltung des Lobau-Vorlandes zu einem attraktiven Puffer-Erholungsgebiet die Besuchsbelastung in der Lobau zu reduzieren. Mögliche Ansatzpunkte für erholungsplanerische Maßnahmen waren die Etablierung einer attaktiven Erholungsinfrastruktur für bestimmte Freizeitaktivitäten in Quantität und Qualität, sowie die Schaffung eines attraktiven Umfeldes für Freizeitaktivitäten im Lobau-Vorland durch ein ansprechendes Landschaftsbild und Landschaftsstrukturen. Diese möglichen Maßnahmen mussten mit den Präferenzen der Erholungssuchenden verglichen werden, um die optimalen Maßnahmen hinsichtlich Erholungsinfrastruktur und Landschaftsbild setzen zu können. Dazu waren die Präferenzen für jeweils spezifische Freizeitaktivitäten zu erfassen (s. Kap. 4.1). Schließlich können Radfahrende andere Präferenzen für die Erholungsinfrastruktur bzw. das Landshcaftbild aufweisen als Hundeausführende. Weiters sollte die Wirksamkeit der Ausgestaltung des Lobau-Vorlandes mittels linear und flächig 
wirkender Maßnahmen der Landschaftsgestaltung hinsichtlich der Pufferkapazität überprüft werden. Als lineare Maßnahmen war z.B. die Anlage von neuen Rad- und Wanderwegen geplant, während flächig wirksame Maßnahmen eine Umwandlung des agrardominierten Lobau-Vorlandes in eine attraktive Erholungslandschaft vorsahen.

\subsubsection{Maßnahmen aus verkehrsplanerischer Sicht}

\subsubsection{Einleitung}

Am Beginn der Überlegungen zur Szenariendefinition standen die geplanten Verkehrsinfrastrukturvorhaben im Umfeld der Lobau und ihre erwartbaren Auswirkungen auf die Besuchesbelastung der Lobau. Aus der Besucherstromanalyse für den Wiener Anteil am Nationalpark Donau-Auen (Arnberger et al., 2000) geht hervor, dass

- $\quad 12 \%$ der Lobau-BesucherInnen zu Fuß,

- $\quad 33 \%$ mit dem Rad,

- $\quad$ nur knapp $6 \%$ mit öffentlichen Verkehrsmitteln, und

- die Mehrheit von knapp $50 \%$ mit dem PKW anreisen.

Bei den über größere Distanzen anreisenden BesucherInnen ist der PKW-Anteil deutlich höher. In der Zone 3 (Region ohne Groß-Enzersdorf und Wien ohne 22. Bezirk) beträgt er $66 \%$, und auch bei den BesucherInnen aus der Zone 2 (22. Bezirk außerhalb des Nahbereichs) liegt er mit $54 \%$ noch vergleichsweise hoch. Dies zeigt die dominante Stellung des PKW bei der Anreise in die Lobau. Es ist demnach zu erwarten, dass Maßnahmen, die die Attraktivität der PKW-Anreise betreffen, deutliche Auswirkungen auf das Ausmaß der Besucherströme haben. Relevante Maßnahmen betreffen daher die Attraktivität des Straßennetzes und die Verfügbarkeit von Parkraum an den Lobaueingängen.

\subsubsection{Maßnahmen im Straßennetz}

Die wichtigste Maßnahme im hochrangigen Straßennetz (Abb. 6) ist die geplante S1 Wiener Außenring Schnellstraße, die Lobauquerung Schwechat - Großenzersdorf. Da ihre Realisierung mit hoher Sicherheit feststeht, eignet sich diese Maßnahme kaum dazu, als Verkehrsszenario verwendet zu werden. Die Erreichbarkeitsanalysen (ÖIR 2007, Netzanalyse Wiener Linien) zeigen, dass die Lobauquerung zwar die Erreichbarkeit der Lobau aus dem südlichen Umland Wiens verbessern wird (Raum Schwechat - Himberg), aus Wien selbst aber nur wenig (ausgenommen die Bereiche entlang der S1-Abschnitt Vösendorf - Schwechat). Da die A23 als Hauptzugangsroute für die Wiener Bevölkerung an den besucherstarken Wochenenden in der Regel nicht überlastet ist, wird diese Maßnahme nur wenig neue Besucherströme für die Lobau anziehen können. Die zweite hochrangige Maßnahme, die Verlängerung der A23 Knoten Hirschstetten - Flugfeld - Stadtgrenze steht in direktem Zusammenhang mit der Realisierung, nunmehr als hochrangige Stadtstraße, wird in der Untersuchung vorausgesetzt; ferner ist auch sie für die Anreise zur Lobau von geringer Bedeutung. 


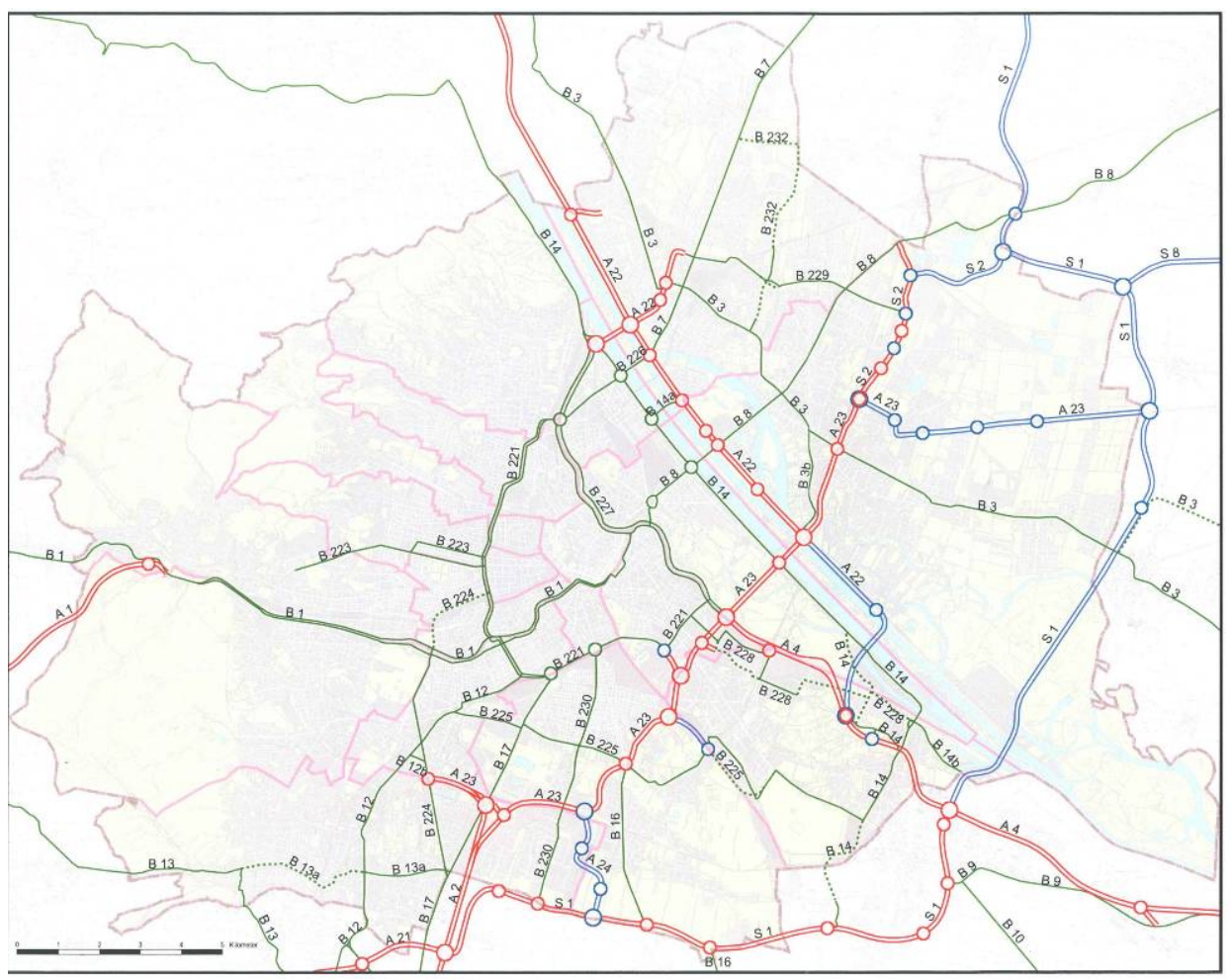

HAUPTSTRASSENNETZ B IN WIEN INKL. BUNDESSTRASSEN A UND S

$=$ Bundesstraben A bzw. $\mathrm{s}$

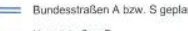

K

Kneten geplant

0

Anschiusstellen nepatart

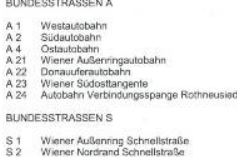

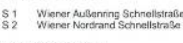

1 Wererer Stabe

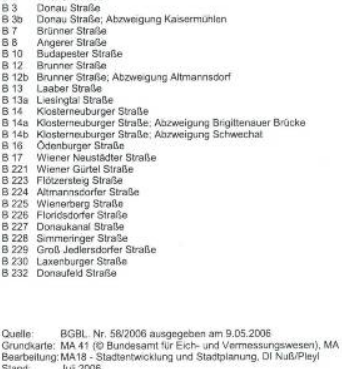

Abb. 6: Hauptstraßennetz B in Wien inkl. Bundesstraßen A und S. Quelle: MA 18

Die dritte hochrangige Maßnahme, die Verlängerung der A22 Knoten Kaisermühlen - A4 Knoten Simmeringer Haide mit Donauquerung beim Roten Hiasl ist derzeit nicht vor 2020 zur Realisierung vorgesehen, der Bedarf dafür soll erst später evaluiert werden (Aussage MA 18, 27.11.2008). Wesentlich ist jedoch, dass diese Maßnahme zwar die stadtseitige Zufahrt zur Lobau etwas verbessern wird, sie in Hinblick auf die Erreichbarkeit der Lobau aber nur einen bescheidenen Gewinn an Reisezeit und Leistungsfähigkeit bringen wird. Weiters sind auch im niederrangigen Netz keine wesentlichen Verbesserungen geplant, die für die Anreise zur Lobau nutzbar sind. Hier achtet die Wiener Stadtplanung (MA 18, 2006, Masterplan Flugfeld Aspern) darauf, dass die Attraktivität des Straßennetzes im sensiblen Lobau-Vorland nicht erhöht wird. Szenarien des Ausbaus des Straßennetzes sind demnach wenig geeignet, unterschiedliche Besucherströme in die Lobau abzubilden.

\subsubsection{Maßnahmen Parkraum}

Ausgangslage

Als wesentliche Stellschraube zur Beeinflussung der Lobau-Anreise bleibt daher die Parkraumsituation. Auch wenn davon ausgegangen wird, dass an den Lobaueingängen noch ausreichend Parkraum vorhanden ist, wird mit der massiven Erhöhung der EinwohnerInnen im LobauVorland eine Änderung der Parkraumsituation notwendig.

Derzeit werden beim Lobauzugang die folgenden Parkmöglichkeiten genutzt:

- entlang Biberhaufenweg, ca. 70 Stellplätze PKW

- Parkplatz Nationalparkzentrum, ca. 150 Stellplätze PKW, 10 - 15 Bus

- Parkplatz Saltenstraße, ca. 100 Stellplätze PKW

- Parkplatz Eßlinger Furt, ca. 20 Stellplätze PKW

- Parkplatz Groß-Enzersdorf, Uferhaus, ca. 100 Stellplätze PKW

- Parkplatz Panozzalacke, mehr als 100 Parkplätze PKW

Von den insgesamt 354 erfassten Stellplätzen an den Lobaueingängen (ohne Raffineriestraße und Panozzalacke) waren am Sonntag den 10.10.2010 352 Plätze (99\%) und am Sonntag den 22.05.11 256 Plätze belegt (72\%, Abb. 7). 


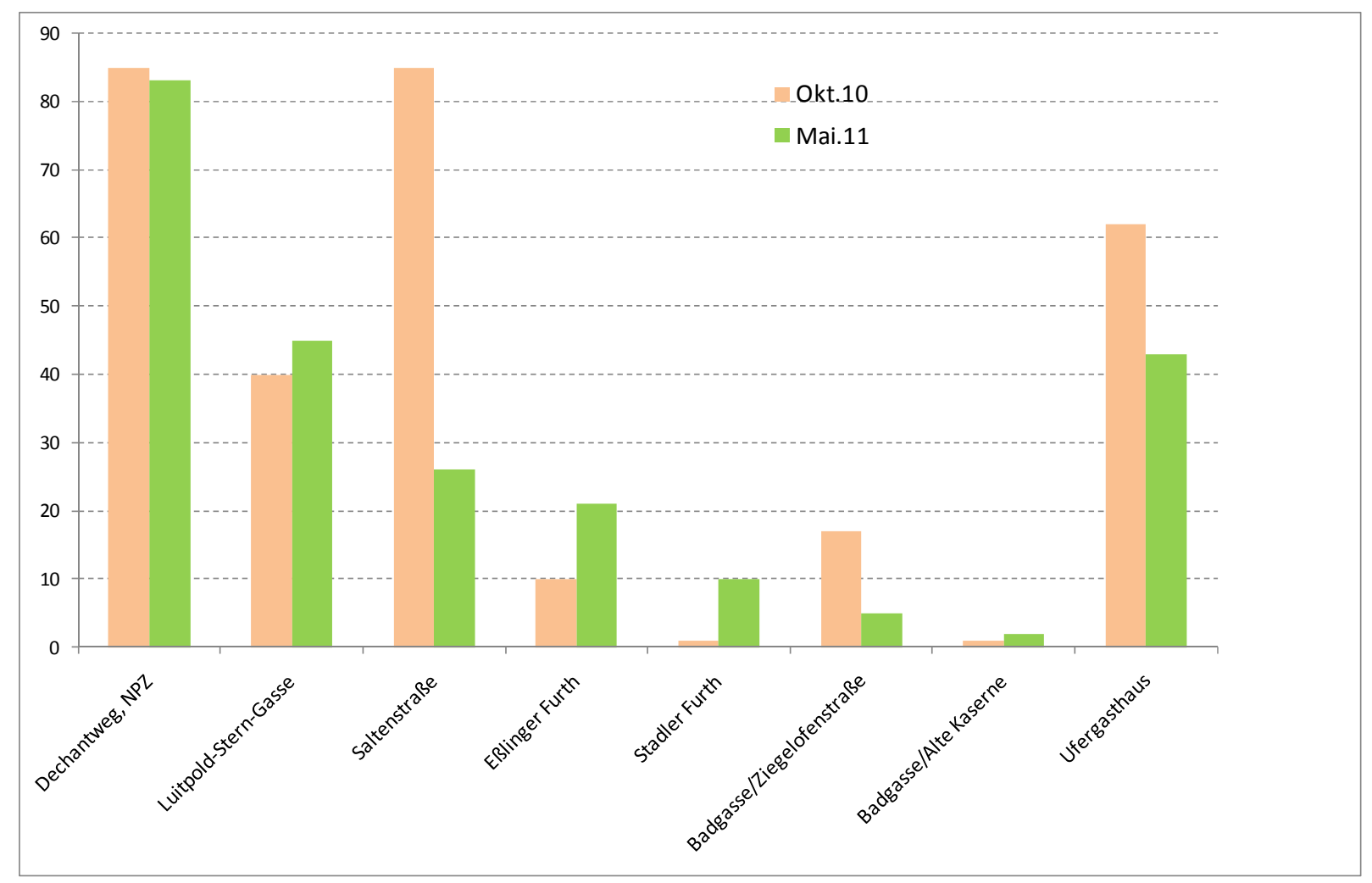

Abb. 7: Stellplatzerhebung Lobaueingänge. Anzahl der abgestellten PKW (11.00 - 12.30). Quelle: ÖIR.

Darüber hinaus stehen Stellplätze entlang der Raffinieriestraße (Zugang Donauinsel) zur Verfügung (mehr als 1.000 Stellplätze). Die Stellplätze in den Katastralgemeinden Mühlleiten sowie Schönau an der Donau sowie teilweise auch in den Siedlungsbereichen des Lobau-Vorlands in Wien und GroßEnzersdorf, deren Straßenquerschnitte meist eng sind, weisen ein schwer quantifizierbares Stellplatzpotenzial auf. Für den unmittelbaren, bequemen Zugang zum Nationalpark Lobau kann somit im Bestand von ca. 500 Stellplätzen ausgegangen werden. Unter Inkaufnahme von längeren Zugangswegen (Raffinieriestraße, Mühlleiten, Schönau), zeitaufwändigerer Parkplatzsuche bzw. möglichem illegalem Abstellen erhöht sich die Anzahl der für den Lobaubesuch verwendbaren Stellplätze auf mehr als 2.000. Möglichkeiten, die Attraktivität der Zufahrt zur Lobau per PKW zu verringern, ergeben sich damit am ehesten durch Zurücknahme und Verkleinerung der unmittelbar am Lobaueingang gelegenen Stellplätze.

\subsubsection{Maßnahmen}

Es zeigen sich somit zwei Möglichkeiten:

Szenario A: Der bestehende Parkraum wird nicht ausgeweitet bzw. sogar verringert. Denkbar sind Maßnahmen zur Parkraumbewirtschaftung an den Lobaueingängen oder die Verlegung der Parkplätze von den Lobaueingängen weg, ggf. wird Parken an den Zufahrtsstraßen untersagt oder bestehende Verbote werden ausgeweitet. Maßnahmen betreffen die Schließung des Schleichwegs Brockhausengasse - Grohmannstraße für PKW sowie die Parkplätze Nationalparkzentrum (Reduzierung der Stellflächen), Saltenstraße (Verlegung 200-300m nördlich) und Panozzalacke (Reduzierung der Stellflächen). In diesem Szenario ist sowohl eine Senkung des PKW-Anteils bei der Anreise zur Lobau als auch ein Rückgang der Gesamtzahl an BesucherInnen zu erwarten. Dieses Szenario bildet eine umweltorientierte Planung ab, wie sie als Ziel der Wiener Stadtplanung (Verringerung des Modal Split von derzeit $35 \%$ auf $25 \%$ MIV-Fahrten) im Masterplan Verkehr 2008 formuliert ist.

Szenario B: Das Parkraumangebot an den Lobaueingängen wird erweitert und an die zunehmende Verkehrsnachfrage angepasst. In diesem Szenario ist sowohl ein Anstieg des PKW-Anteils bei der Anreise zur Lobau als auch ein Anstieg der Gesamtzahl an BesucherInnen zu erwarten. Szenario B 
spiegelt die Praxis des Laissez-faire ab, wie sie bislang zumindest am Stadtrand häufig anzutreffen war.

\subsubsection{Maßnahmen öffentlicher Verkehr}

Auch wenn der ÖV bei den Lobau-BesucherInnen derzeit geringe Anteile und bei den aus dem Nahbereich in die Lobau anreisenden BesucherInnen kaum Anteile hat, könnte der öffentliche Verkehr künftig eine etwas stärkere Rolle im Zugang zur Lobau erhalten (Abb. 8). Einerseits durch die bereits 2010 erfolgte Verlängerung der U2 bis Aspernstraße, die die Anreise aus den inneren Bezirken Wiens im ÖV bereits deutlich verbessert hat, sowie durch deren weiterer Verlängerung bis zum Flugfeld Aspern (Inbetriebnahme geplant für 2013). Die Maßnahme ist integraler Bestandteil der Siedlungsentwicklung am Flugfeld, daher nicht szenariofähig.

Mit der U2 Verlängerung ist ein abgestimmtes Sekundärnetz mit zahlreichen Verbesserungen - neuen Linienführungen und Intervallverdichtungen - geplant. Bestehende Buslinien werden verstärkt an die U2 angebunden, wobei die Anbindungen in den ÖV-Knoten der U2-Stationen Stadlau, Donauspital, Aspernstraße, Hausfeldstraße und Flugfeld Nord erfolgen.

Hauptaugenmerk wird auf die verbesserte Erreichbarkeit in Richtung Stadt gelegt, die Verbesserungen zur Erreichbarkeit der Lobau sind gering. $\mathrm{Zu}$ nennen ist eine verbesserte Busanbindung zum Biberhaufenweg durch die Linie 93A (U2 Aspernstraße - Biberhaufenweg - U2 Kaisermühlen VIC; dichtere Intervalle). Weiters wird die entlang dem Lobau-Vorland verkehrende Linie 98A verdichtet, was die Erreichbarkeit der Eßlinger Furt aus Aspern verbessert.

Direkte Buslinien zwischen dem Flugfeld und der Lobau sind nach dem derzeitigen Stand der Planung nicht vorgesehen. Eine wirkungsvolle Maßnahme wäre daher eine eigene „Lobau-Linie“. Die Einrichtung einer solchen Linie wird in Szenario A angenommen (Flugfeld Nord S U - Flugfeldstraße - Grohmannstraße - Saltenstraße - Lobaueingang - Brockhausengasse - Asperner Heldenplatz, Anschluss an Straßenbahnlinie 25).

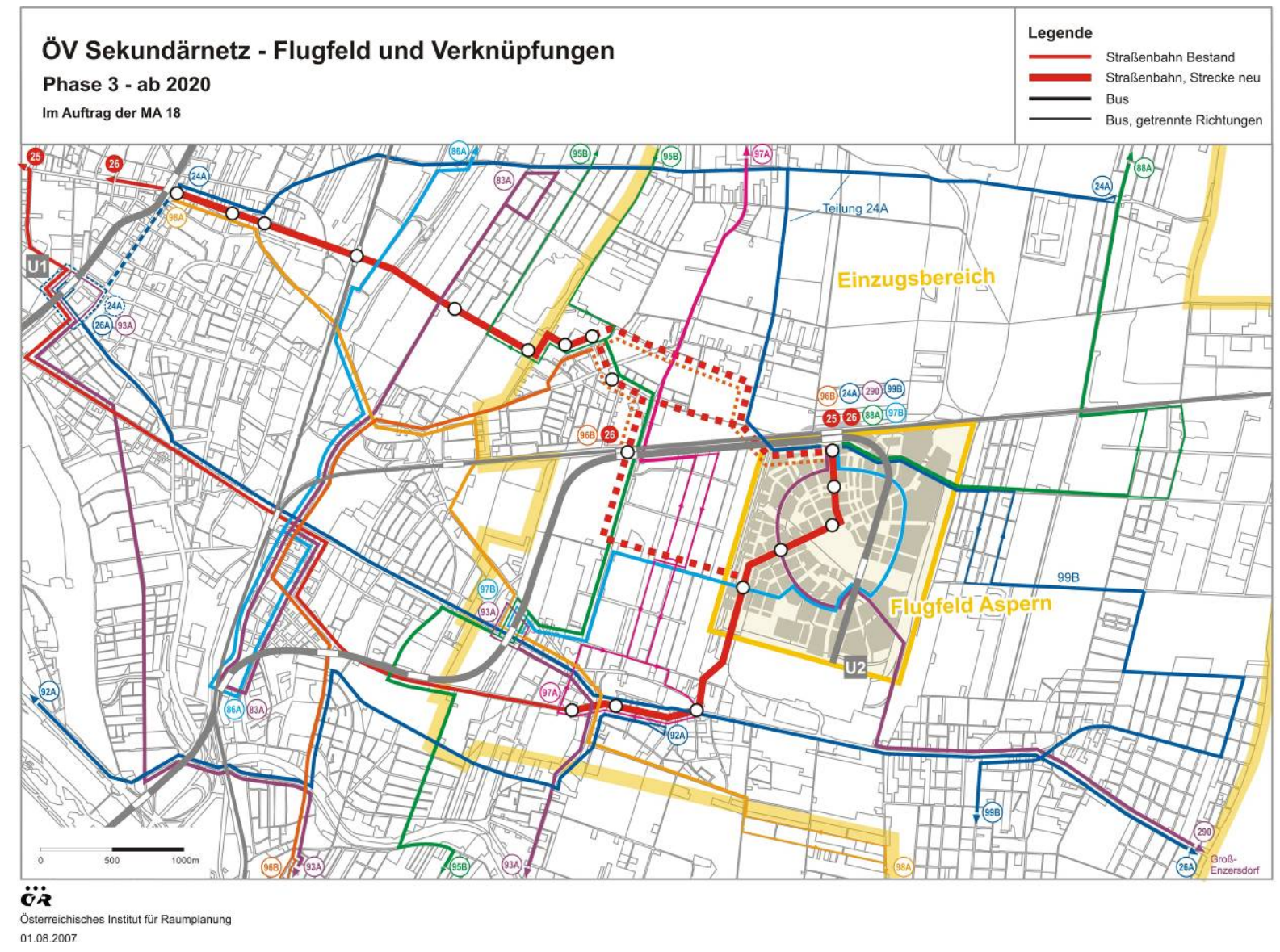

Abb. 8: Öffentliche Verkehrsanbindung Flugfeld Aspern. Quelle: ÖIR, 2006. Sekundärnetz Flugfeld. Auftrag der MA18. 


\subsection{Workshops mit Stakeholdern}

Gemeinsam mit Stakeholdern wurden auf Basis der ersten entwickelten Maßnahmen die Szenarien (Maßnahmenbündel) formuliert. Dazu wurden zwei Workshops veranstaltet. Der erste StakeholderWorkshop fand am 28.01.2009 und der zweite am 9. März 2010 statt (s. Abb. 9 und Abb. 10). VertreterInnen folgender Institutionen nahmen am Workshop teil: Nationalpark Donauauen GesmbH, MA 49 - Forstamt und Landwirtschaftsbetrieb der Stadt Wien - Forstverwaltung Lobau, Stadtteilplanung und Flächennutzung Süd-Nordost (MA 21B, Wien), Magistratsabteilung 22 Umweltschutz (MA 22, Wien), Planungsgemeinschaft Ost (PGO), Stadt Umland Management (SUM) und der Stadtentwicklung und Stadtplanung (MA 18, Wien). Aus terminlichen Gründen abgesagt hatten VertreterInnen der Gemeinde Groß-Enzersdorf.

Im Rahmen der Workshops wurden Szenarien für die Lobau im Jahr 2020/2025 entwickelt und Maßnahmen diskutiert, die den zu erwartenden steigenden Besucherdruck kompensieren bzw. verringern könnten. Einig waren sich die Stakeholder darin, dass die Attraktivität bzw. Erreichbarkeit der Lobau gesenkt werden sollte und als Ausgleich dazu die Qualität des Lobau-Vorlandes gehoben werden müsste. Der zweite Workshop diente der Verfeinerung der im Workshop von Jänner 2009 entwickelten Szenarien. So wurde eine mögliche Radroute um die Lobau planlich verortet (Abb. 11). Bisherige Forschungsergebnisse wurden präsentiert und diskutiert.

Im dritten Workshop im Mai 2011 wurden dann die Ergebnisse der Simulationen und mögliche Maßnahmen v.a. auf Stadtplanungsebene diskutiert. 


\title{
PROGRAMM
}

für Mittwoch, den 28. Jänner 2009

\author{
14.00 Uhr Begrüßung \\ Gregori Stanzer \\ 14.00 - 14.20Uhr Perspektive Lobau 2020 - Das Projekt \\ Arne Amberger, ILEN
}

14.20 - 15.20 Uhr Lobau 2020: Ohne Perspektive?

Was die Zukunft bringt ...

Statements zur Entwicklung von

- Siedlungen

- Verkehr

- Erholungsnutzung

- Ōkologie: Gewässer, Pflanzen (inkl. Forst-/Landwirtsch.). Tiere

Statements der Projektpartner und der Experten der Behörde

Diskussion im Plenum

15.20 - 16.45 Uhr Lobau 2020: Auf der Suche nach Perspektiven

Mögliche Maßnahmen ...

Impulsreferate mit anschlieBender Diskussion in

Arbeitsgruppen zu

- Siedlungs- und Verkehrsentwicklung

- Erholungsnutzung und Ökologie

Szenarien der Lobau 2020 ...

Diskussion im Plenum

$16.45-17.00 \mathrm{Uhr}$ Resūmee

Landkarte der Szenarien

Arne Amberger, ILEN

Moderation: Gregori Stanzer, ÖIR

Ort: $\quad$ ÖR, 1010 Wien, Franz-Josefs-Kai 27 (Konferenzzimmer)

Perspektive Lobau 2020

Seit Sommer 2008 wird im Rahmen des MAB-Projekts „Perspektive Lobau 2020" untersucht, wie wirksam verschiedene MaBnahmen im Umgang mit dem im Jahre 2020 zu erwartenden Besucherdruck auf den Biospharenpark Lobau sind. Die Auswirkungen verschiedener MaBnahmen innerhalb und auBerhalb des Biospharenparks werden in Bezug auf die Anwohner, auf die Erholungsqualitat for Erholungssuchende und auf die Okologie der Lobau betrachtet.

Das Projekt „Perspektive Lobau 2020" ist auch vor dem Hintergrund der neuen Seestadt Wiens - dem ehemaligen Flugfeld Aspern - einer verbesserten Verkehrsanbindung und einer dynamischen Entwicklung des Stadtgebietes in unmittel barer Nahe zur Lobau zu sehen.

Im Szenarienworkshop am 28. Janner wird die Entwicklung in und um die Lobau bis zum Jahre 2020 skjzziert, werden mogliche Maßnahmen für die Lobau im Umgang mit dieser Entwicklung diskutiert SchlieBlich werden jene Zukunfts-Szenarien ausgewahlt, welche im Rahmen des Projektes „Perspektive Lobau 2020" noch naher untersucht werden.

Abb. 9: Programm des 1. Stakeholder-Workshops 


\section{PROGRAMM}

für Dienstag, den 9. März 2010

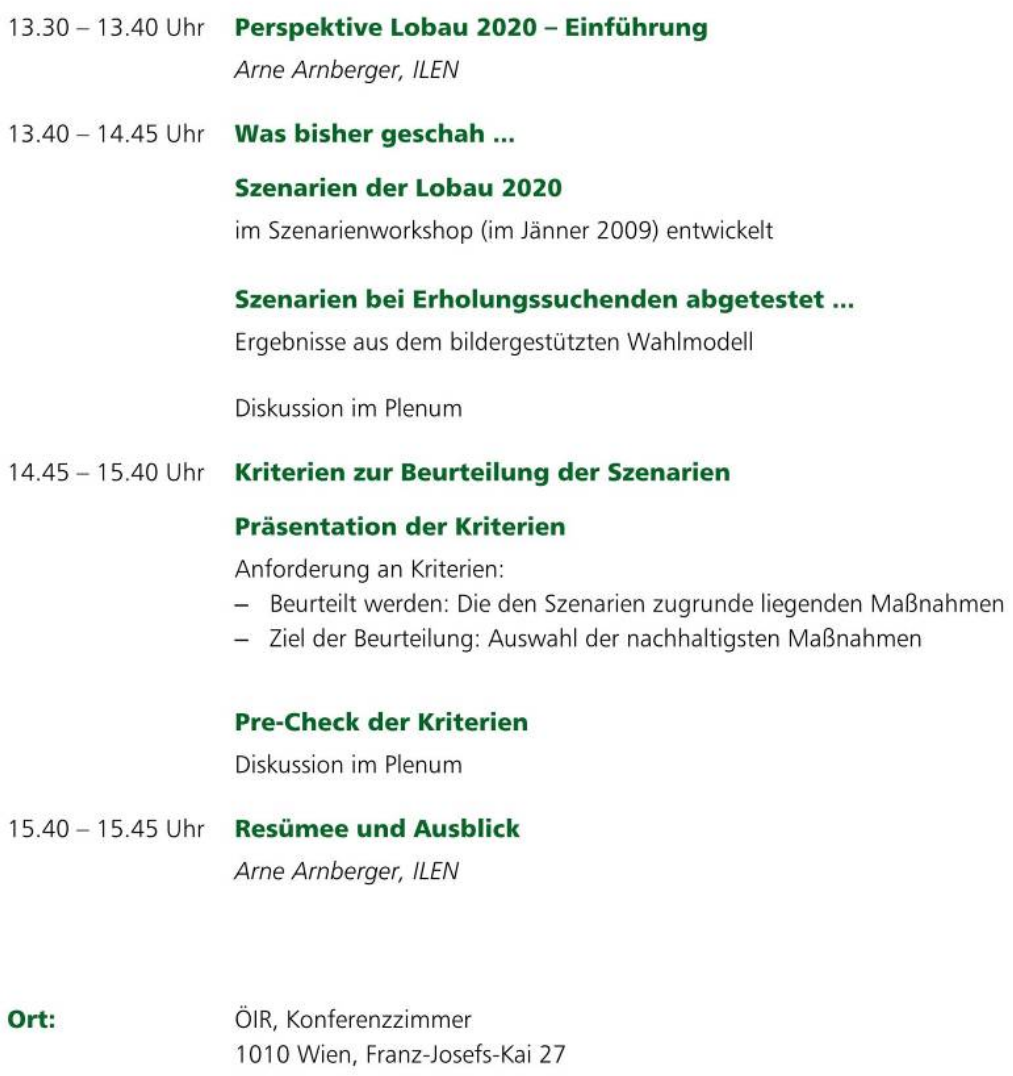

Das MAB-Projekt „Perspektive Lobau 2020" untersucht, wie wirksam verschiedene Maßnahmen im Umgang mit dem zu erwartenden Besucherdruck auf den Biosphärenpark Lobau sind. Dafür werden verschiedene Maßnahmen berücksichtigt und deren Wirksamkeit untersucht.

Im Stakeholderworkshop im März 2010 werden bisherige Ergebnisse vorgestellt: Die im Szenarienworkshop (Jänner 2009) entwickelten Szenarien werden präsentiert, die Ergebnisse aus dem bildergestützten Wahlmodell werden diskutiert. Mit diesem Wahlmodell wurde das Verhalten von Erholungssuchenden bei unterschiedlichen Szenarien abgefragt.

Schließlich werden jene Kriterien ausgewählt, mit denen es gelingen soll, die nachhaltigsten Maßnahmen auszuwählen.

Abb. 10: Programm des 2. Stakeholder-Workshops 


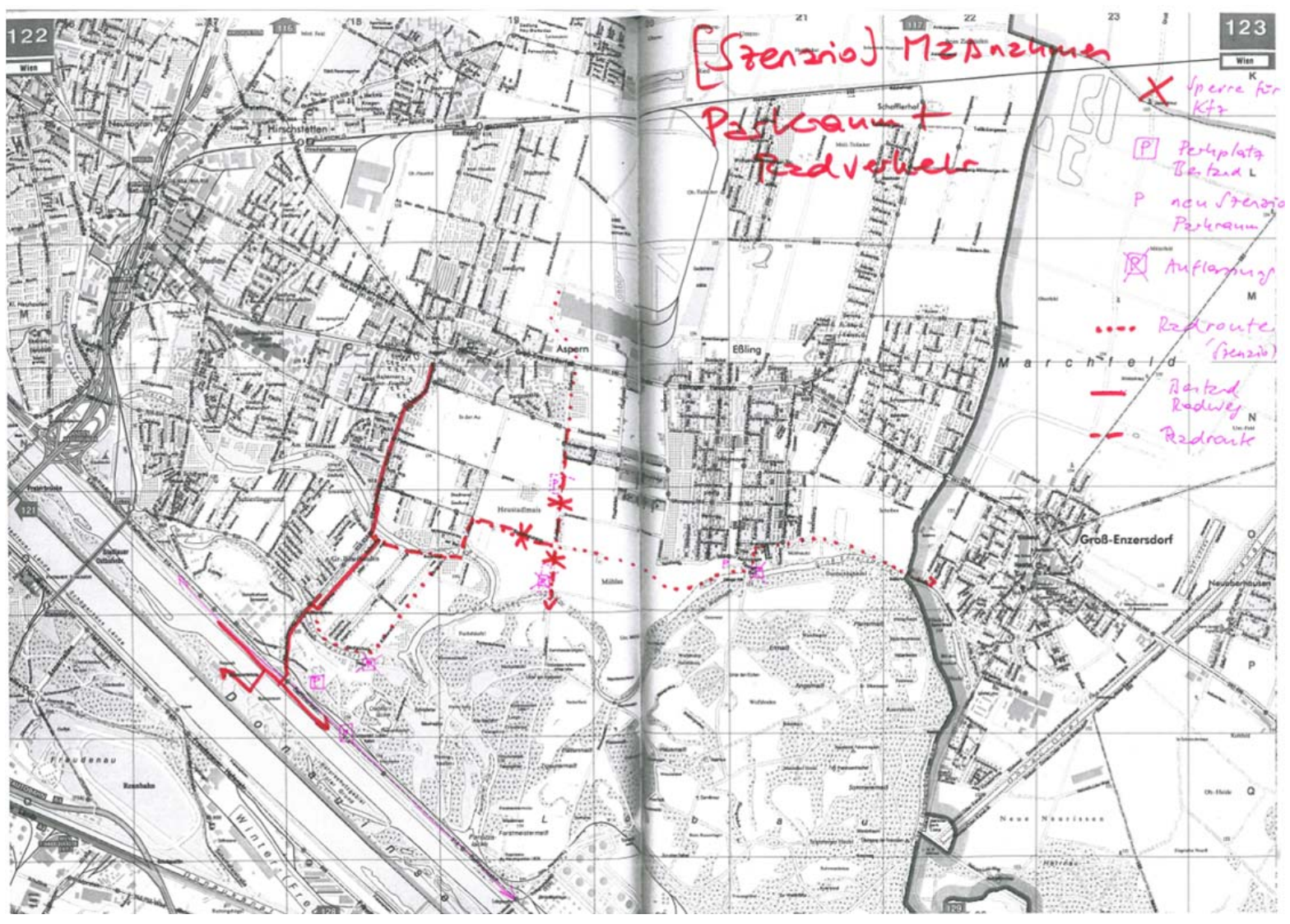

Abb. 11: Radwegkarte mit Vorschlägen zu Radwegführungen im Lobau-Vorland basierend auf dem 2. Stakeholder Workshop

\subsection{Definierte Szenarien}

Die nachfolgend angeführten Szenarien basieren auf den Ergebnissen der Stakeholderworkshops sowie den Projektpartnertreffen. Die Maßnahmen in der Lobau sollten v.a. auf eine Beeinflussung von RadfahrerInnen (größte Lobau-Nutzergruppe mit $47 \%$ ) und HundebesitzerInnen (Anteil knapp 10 \%; größter Einfluss auf den Naturraum, da Hunde nicht an der Leine gehalten werden) abzielen, aber auch eine ökologische Verbesserung (Gewässerszenario Offen $1 \mathrm{~b}$ verlandet aus dem Projekt Optima Lobau, Abb. 5) miteinbeziehen.

Die Maßnahmen im Lobau-Vorland hatten folgende Zielsetzungen:

- Ausgestaltung des Vorlandes mittels linear und flächig wirksamer Maßnahmen der Landschaftsgestaltung

- Attraktivitätssteigerung für die Aktivität Radfahren im Vorland (lineare Maßnahmen, Definition von neuen Radwegen im Lobau-Vorland, die um und nicht in die Lobau führen)

- Attraktivitätssteigerung des Vorlandes für Hundeausführende

- Parkraummanagement (Verlegung von Parkplätzen von der Lobau weg, Auflassung von Parkplätzen)

Im Folgenden wurden sechs Basisszenarien definiert und simuliert. Als Basis zur Bemessung der Auswirkungen der Maßnahmenbündel v.a. in Hinblick auf die Besucherzahlreduktion in der Lobau wurde das Szenario „2025“ als „Laissez-faire -Szenario“ definiert. Dieses beschreibt eine Situation, wo keinerlei Maßnahmen getroffen werden und somit die prognostizierte Besucherzahlentwicklung voll wirksam auf die Lobau wird. Ein weiteres Szenario inkludierte die Anbindung des Hauptgewässerzuges der Unteren Lobau an die Donau, die zu einer deutlich erhöhten Gewässerdynamik und zur Förderung von auentypischen Standorten führen wird.

Die Definition der weiteren vier Basisszenarien gliederte sich in Maßnahmen im Lobau-Vorland und in der Lobau. Die Maßnahmen im Lobau-Vorland untergliederten sich in eine lineare und flächige Ausgestaltung des Vorlandes. Jene in der Lobau beinhalteten entweder die derzeitige 
Gewässersituation oder eine Anbindung des Hauptgewässerzuges der Unteren Lobau an die Donau (Gewässerszenario 1b).

Die vier Basisszenarien enthielten ein ganzes Bündel an Maßnahmen (Tab. 8). In die Formulierung der Szenarien flossen auch Ergebnisse der BesucherInnenbefragung hinsichtlich ihrer Landschafts- und Erholungsinfrastrukturpräferenzen ein (s. Kap. 4.1).

Die Bezeichnung „Lobau A/B“ beinhaltet keine (A) bzw. eine Anbindung (B) der Unteren Lobau an die Donau. Der Begriff „Vorland A“ beschreibt eine lineare Ausgestaltung des Lobau-Vorlandes. Darunter ist die Anlage eines Radwegenetzes tlw. auf bestehenden Straßen, die für den motorisierten Verkehr (außer AnwohnerInnen) zu sperren sind, zu verstehen. Diese Wege sind breit, geschottert oder asphaltiert und von einer Allee gesäumt. Dieses neue Radwegenetz soll v.a. ein Hineinfahren in die Lobau für die Radfahrenden weniger attraktiv machen. Vielmehr sollten sie motiviert werden, um die Lobau zum Erholungsgebiet Neue Donau zu fahren. Einige Parkplätze werden von der Lobau weg verlegt, um den Besuchswiderstand zu erhöhen und um die BesucherInnen zu zwingen, auch die neuen Wege des Vorlandes zu benutzen (Eingänge Saltenstraße, Esslinger Furt). Der Parkplatz LuitpoltsternGasse wird aufgelassen.

Der Begriff „Vorland B“ beschreibt eine flächige Ausgestaltung des Lobau-Vorlandes mit einer WaldWiesen-Landschaft sowie das in Vorland A beschriebene Radwegenetz, welches zusätzlich durch ein Wanderwegenetz mit schmäleren Wegen, die von Alleen gesäumt sind, ergänzt wird. Damit sollte ein ähnlich engmaschiges Wegegenetz wie in der Lobau angeboten werden. Das Parkraummanagement entspricht jenem für das „Vorland A“.

\begin{tabular}{|l|c|c|c|c|c|c|}
\hline \multicolumn{1}{|c|}{ Maßnahmen } & $\mathbf{2 0 2 5}$ & $\begin{array}{c}\mathbf{2 0 2 5} \text { mit } \\
\text { 1b }\end{array}$ & $\begin{array}{c}\text { Lobau A } \\
\text { Vorland } \\
\text { A1 }\end{array}$ & $\begin{array}{c}\text { Lobau B } \\
\text { Vorland A }\end{array}$ & $\begin{array}{c}\text { Lobau A } \\
\text { Vorland } \\
\text { B2 }\end{array}$ & $\begin{array}{c}\text { Lobau B } \\
\text { Vorland B }\end{array}$ \\
\hline Besucheranzahl 2025 & $\mathrm{X}$ & $\mathrm{X}$ & $\mathrm{X}$ & $\mathrm{X}$ & $\mathrm{X}$ & $\mathrm{X}$ \\
\hline Gewässerszenario 1b & & $\mathrm{X}$ & & $\mathrm{X}$ & & $\mathrm{X}$ \\
\hline $\begin{array}{l}\text { Neue breite Wege mit } \\
\text { Alleen im Vorland für } \\
\text { RadfahrerInnen }\end{array}$ & & & $\mathrm{X}$ & $\mathrm{X}$ & $\mathrm{X}$ & $\mathrm{X}$ \\
\hline $\begin{array}{l}\text { Parkraummanagement } \\
\text { an den Eingängen } \\
\text { Saltenstraße, Esslinger } \\
\text { Furt, Luitpoltstern- } \\
\text { Gasse }\end{array}$ & & & $\mathrm{X}$ & $\mathrm{X}$ & $\mathrm{X}$ & $\mathrm{X}$ \\
\hline $\begin{array}{l}\text { Neues Wegenetz mit } \\
\text { schmalen Wegen und } \\
\text { Alleen im Vorland für } \\
\text { FußgängerInnen }\end{array}$ & & & & & $\mathrm{X}$ & $\mathrm{X}$ \\
\hline $\begin{array}{l}\text { Flächige Ausgestaltung } \\
\text { (Wald/Wiese) des } \\
\begin{array}{l}\text { Vorlandes im Bereich } \\
\text { Saltenstraße }\end{array}\end{array}$ & & & & & $\mathrm{X}$ & $\mathrm{X}$ \\
\hline
\end{tabular}

Tab. 8: Definierte Szenarien als Maßnahmenbündel (1) 2025 fokussiert auf Radfahrende; (2) fokussiert auf Radfahrende/HundebesitzerInnen

Zur Bewertung der Szenarien wurden Kriterien aus den Bereichen der Erholungsplanung, Stadt- und Verkehrsentwicklung und der Ökologie von aquatischen und terrestrischen Systemen sowie der Wildtierökologie definiert. Die Kriterien wurden flächenbasiert auf Rasterbasis für die gesamte Lobau dargestellt. Mit Hilfe der Kriterien konnten die Szenarien detailliert bewertet (Risikoanalyse) und verglichen werden. 


\section{Bestimmung der Eingangsparameter als Basis für die Simulation des Besucherverhaltens}

\subsection{Modellierung des Besucherverhaltens anhand eines Wahlmodells}

Ziel des Wahlmodells war es, die Attraktivität der Lobau zur Bestandssituation des Lobau-Vorlandes, als auch zu möglichen Szenarien der Ausgestaltung des Vorlandes in Relation zu setzen. Damit konnte erfasst werden, inwieweit eine bestimmte Ausgestaltung des Lobau-Vorlandes ähnlich attraktiv oder sogar attraktiver als die Lobau selbst ist. Anhand eines visuellen Wahlmodells (Arnberger \& Eder, 2009; Arnberger et al., 2010; Louviere et al., 2000; Reichhart \& Arnberger, 2010) wurden wichtige Eingangsparameter für die agenten-basierte Simulation erfasst. Mittels dieser agenten-basierten Simulation können räumliches Entscheidungsverhalten in Abhängigkeit von sozialen, physischen und management-bezogenen Faktoren simuliert, und die Pufferkapazität möglicher Maßnahmenszenarien berechnet werden. Gleichzeitig zeigt die Simulation die räumliche Verteilung der erholungssuchenden Agenten auf. Dies lässt Rückschlüsse auf Belastungssituationen für den Naturraum zu.

\subsubsection{Design des Wahlmodells}

Für das Design des Wahlmodells waren folglich zwei Ebenen zu berücksichtigen (Tab. 9). Eine Ebene betraf die Bestandssituation, da sowohl die wichtigsten Charakteristika der Lobau als auch die des Lobau-Vorlandes darzustellen waren. Die zweite Ebene betraf die verschiedenen Gestaltungs- und Managementszenarien, die untersucht werden sollten. Diese beziehen sich wiederum sowohl auf die Lobau als auch auf das Lobau-Vorland.

\begin{tabular}{|l|l|l|}
\hline \multicolumn{1}{|c|}{ Räumliche Ebene } & \multicolumn{2}{|c|}{ Bestandes - Maßnahmen-Ebene } \\
\hline Lobau & Bestand & Szenarien: \\
& Landschaft & Gewässerdynamik; \\
& Gewässer & Wege (Anzahl, Länge, Stich- \\
& Wege ... & bzw. Rundwege) ... \\
\hline Lobau-Vorland & Bestand & Szenarien: \\
& Landschaft & Landschaft \\
& Wege ... & Wege \\
& & Verkehr ... \\
\hline
\end{tabular}

Tab. 9: Ebenen des Wahlmodells

Die Gestaltungs- und Managementszenarien betrafen:

- Ökologische Maßnahmen

- Erholungsbezogene Maßnahmen (Erholungsinfrastruktur, Landschaftsästhetik)

- Verkehrsbezogene Maßnahmen

Zur Erfassung der Präferenzen der BesucherInnen hinsichtlich Erholungsinfrastruktur, Landschaft und Besuchermengen wurde ein Design erstellt, welches elf Variablen mit verschiedenen Abstufungen enthielt (Tab. 10). Diese Variablen beschrieben physische, managementbezogene und soziale Parameter, die wiederum sowohl den Bestand als auch die Gestaltungs- und Managementszenarien beschrieben. Diese können in die Ebenen

(a) Erholungsweg (Breite, Belag, Anzahl Wegnutzer am Weg, Anzahl Hunde am Weg, Wegebegleitgrün...),

(b) Landschaft (entlang des Weges, wie Wald, Wiesen, Heißländen, Gewässertypen) und

(c) Attraktionen (z.B. Wildbadeplatz) und Lenkung (Beschilderung) untergliedert werden. 
a) Erholungsweg:

Der Weg stellt eine zentrale Ebene des Wahlmodells dar. Diese Ebene enthält sowohl soziale wie auch physische Parameter. Die drei sozialen Parameter beschreiben die Anzahl der BesucherInnen, die am Weg dargestellt sind, die Art der Aktivitäten, denen die Erholungssuchenden nachgehen, und das Besucherverhalten. Das Besucherverhalten wurde anhand von Hunden, die an oder nicht an der Leine geführt wurden, erfasst. Mit der Menge der BesucherInnen am Weg werden crowding-Effekte untersucht. Vor allem zu viele Wegnutzende können ein Grund für Erholungssuchende sein, einen Weg nicht aufzusuchen. Da Radfahrende und zu Fußgehende, inklusive der Hundausführenden, knapp $97 \%$ der Erholungsbesuche ausmachen (Arnberger et al., 2000), wurden nur diese Nutzergruppen hineingenommen. Hundeausführende verursachen knapp $10 \%$ aller Besuche. Ungefähr 50.000 Hunde werden im Jahr in die Lobau hineingeführt, wobei nur ein Drittel bis zur Hälfte der Hunde angeleint ist.

Die drei physischen Parameter beschreiben Wegbreite, Wegebelag sowie Begleitbepflanzung am Wegrand (z.B. Baumallee). Damit wurden die wichtigsten vorkommenden Wegetypen der Lobau als auch jene des Lobau-Vorlandes dargestellt. Gerade für jene Szenarien, die die Radfahrenden betreffen (Szenarien 1 und 3), kann damit die Attraktivität eines linearen Elementes simuliert werden.

b) Landschaft:

Diese Ebene beschreibt die umgebende Landschaft des Erholungsweges. Hier werden Landschaftstypen dargestellt, die in der Lobau bzw. im Lobau-Vorland vorkommen. Oftmals finden sich in der Lobau links und rechts des Weges unterschiedliche Landschaftstypen. Daher wurden auch Kombinationsmöglichkeiten verschiedener Landschaftstypen in das Design aufgenommen, beispielsweise Wald mit Wiese oder Heißlände mit Wiese. Im Laufe der letzten Jahre wurden einige Ackerflächen in der Lobau auf- und der natürlichen Sukzession überlassen. Künftig sollen - nach Aussage des Gebietsmanagements - alle noch bestehenden Ackerflächen in der Lobau aufgelassen werden. Folglich werden Sukzessionsflächen relativ häufig in der Lobau anzutreffen sein. Sie wurden daher in das Wahlmdell aufgenommen. Als weiterer Landschaftsparameter wurde der Ausblick in Gehrichtung definiert. Damit konnte der vor den BesucherInnen liegende Horizont modelliert werden, da das Horizonterlebnis gerade im Lobau-Vorland aufgrund der Offenheit der Agrarlandschaft eine wichtige Rolle darstellt.

\section{c) Attraktionen und Lenkung}

Neben dem Weg und seiner umgebenden Landschaft spielen Ziele/Attraktionen entlang des Erholungsweges sowie indirekte Lenkungsmaßnahmen eine Rolle bei der Wegewahl. Gewässer wurden als Attraktion definiert. Gewässer in der Lobau, zumeist kleinere und größere Altarme, sind hauptsächlich als punktuelle Ausblicke erlebbar und wurden daher bildlich im Wahlmodell dargestellt. Um den Zusammenhang zwischen Gewässerdynamik, Landschaftswandel und Landschaftspräferenzen aufzuzeigen (s. Kapitel 4.1), wurden die Fotos zweier Altarmtypen mit unterschiedlichen Graden an Schilfbewuchs dargestellt. Lenkungsmaßnahmen wurden sowohl über die Wegebeschilderung, als auch durch die Bereitstellung von Parkplätzen bzw. Haltestellen des ÖPNVs simuliert. 


\begin{tabular}{|c|c|}
\hline Parameter & Parameterabstufungen \\
\hline \multicolumn{2}{|l|}{ A) Ebene Weg } \\
\hline 1) Wegbreite & $\begin{array}{l}\text { 1.1) } 0,8 \mathrm{~m} \\
\text { 1.2) } 2 \mathrm{~m} \\
\text { 1.3) } 3 \mathrm{~m} \\
\text { 1.4) } 4 \mathrm{~m}\end{array}$ \\
\hline 2) Wegbelag & $\begin{array}{l}\text { 2.1) Asphalt } \\
\text { 2.2) Schotter } \\
\text { 2.3) Erde mit grünem Mittelstreifen } \\
\text { 2.4) Erde mit grünem Mittelstreifen und Pfützen }\end{array}$ \\
\hline 3) Wegpflanzung & $\begin{array}{l}\text { 3.1) keine Wegbegleitbepflanzung } \\
\text { 3.2) Baumreihe } \\
\text { 3.3) Allee } \\
\text { 3.4) Einzelbaum am Weg }\end{array}$ \\
\hline 4) Besuchermenge & $\begin{array}{l}\text { 4.1) } 1 \text { Person } \\
\text { 4.2) } 4 \text { Personen } \\
\text { 4.3) } 8 \text { Personen } \\
\text { 4.4) } 16 \text { Personen }\end{array}$ \\
\hline 5) Nutzergruppen & $\begin{array}{l}\text { 5.1) } 100 \% \text { FußgängerInnen (Rad verboten) } \\
\text { 5.2) } 75 \% \text { FußgängerInnen, } 25 \% \text { RadfahrerInnen } \\
\text { 5.3) } 50 \% \text { RadfahrerInnen, } 50 \% \text { FußgängerInnen } \\
\text { 5.4) } 75 \% \text { RadfahrerInnen, } 25 \% \text { FußgängerInnen }\end{array}$ \\
\hline 6) Hunde & $\begin{array}{l}\text { 6.1) keine Hunde } \\
\text { 6.2) } 2 \text { Hunde an der Leine } \\
\text { 6.3) } 2 \text { Hunde nicht an der Leine } \\
\text { 6.4) } 1 \text { Hund nicht an der Leine }\end{array}$ \\
\hline \multicolumn{2}{|l|}{ B) Ebene Landschaft } \\
\hline 7) Landschaftstypus & $\begin{array}{l}\text { 7.1) Wald } \\
\text { 7.2) Wiese } \\
\text { 7.3) Heißlände } \\
\text { 7.4) Acker } \\
\text { 7.5) Acker \& Wiese } \\
\text { 7.6) Wald \& Heißlände } \\
\text { 7.7) Wiese \& Wald } \\
\text { 7.8) Sukzessionsfläche \& Wald }\end{array}$ \\
\hline 8) Hintergrund - Horizont & $\begin{array}{l}\text { 8.1) Waldkulisse ohne Strommast } \\
\text { 8.2) Waldkulisse mit Strommast }\end{array}$ \\
\hline \multicolumn{2}{|c|}{ C) Ebene Attraktionen und Lenkung } \\
\hline 9) Gewässer - Attraktionen & $\begin{array}{l}\text { 9.1) Kein Ausblick auf Gewässer } \\
\text { 9.2) Altarm klein mit } 0 \% \text { Schilfbedeckung } \\
\text { 9.3) Altarm klein mit } 50 \% \text { Schilf-Bedeckung } \\
\text { 9.4) Altarm groß mit } 0 \% \text { Schilf-Bedeckung } \\
\text { 9.5) Altarm groß mit } 50 \% \text { Schilf-Bedeckung } \\
\text { 9.6) Altarm groß mit } 100 \% \text { Schilf-Bedeckung }\end{array}$ \\
\hline 10) Infrastruktur-Attraktion & $\begin{array}{l}\text { 10.1) keine Attraktionen am Weg } \\
\text { 10.2) Große Hundezone mit Sitzmöglichkeiten } \\
\text { 10.3) Wildbadeplatz mit Liegewiese oder Wildfreigehege } \\
\text { 10.4) Gasthaus mit Gastgarten }\end{array}$ \\
\hline 11) Anreise & $\begin{array}{l}\text { 11.1) Kein Parkplatz für PKW, keine ÖV-Hst am Wegbeginn/ende } \\
\text { 11.2) Wegebeschilderung an Kreuzungspunkten (Bilder) } \\
\text { 11.3) Parkplatz für PKW } \\
\text { 11.4) ÖV-Haltestelle }\end{array}$ \\
\hline
\end{tabular}

Tab. 10: Design des Wahlmodells 


\subsubsection{Erstellung des Erhebungsinstruments und des Wahlmodells}

Für das Wahlmodell wurden 256 Szenarien generiert. Grundlage für die Szenariengenerierung bildete ein orthogonales fractional factorial Design (Louviere et al., 2000). Da digital kalibrierte Bilder zum Einsatz kommen sollten (Arnberger \& Haider, 2007), wurden ausgehend von einem Bild alle anderen Szenarien in dieses Bild mittels Bildmanipulationssoftware „hineingesetzt“. Als Basis für die Bildererstellung wurde eine ausführliche Fotodokumentation über die in der Lobau und im LobauVorland vorkommenden Landschafts- und Wegetypen erstellt. Der Kamerawinkel blieb bei den einzelnen Aufnahmen immer gleich. Die Darstellung der Szenarien erfolgte aus der Perspektive eines/r Wegenutzers/in. Auch der Schattenwurf wurde berücksichtigt.

Als Hintergrundbild wurde ein $2 \mathrm{~m}$ breiter und $150 \mathrm{~m}$ langer Schotterweg in der Oberen Lobau ausgewählt, der auf eine Waldkulisse zuläuft (siehe Abb. 12). Damit die Größenverhältnisse der dargestellten Personen auf dem Weg auch der Realität entsprechen, wurde eine Fotoserie erstellt, die Menschen in definierten Abständen zeigt. Weiters wurden viele Wegnutzende fotografiert, um „Dummies“ für die Darstellung der sozialen Faktoren zu haben. Anschließend wurde ein Aufstellungsplan erstellt, der definiert, in welchen Abständen die Personen auf den Weg zu stellen sind (Abb. 13).

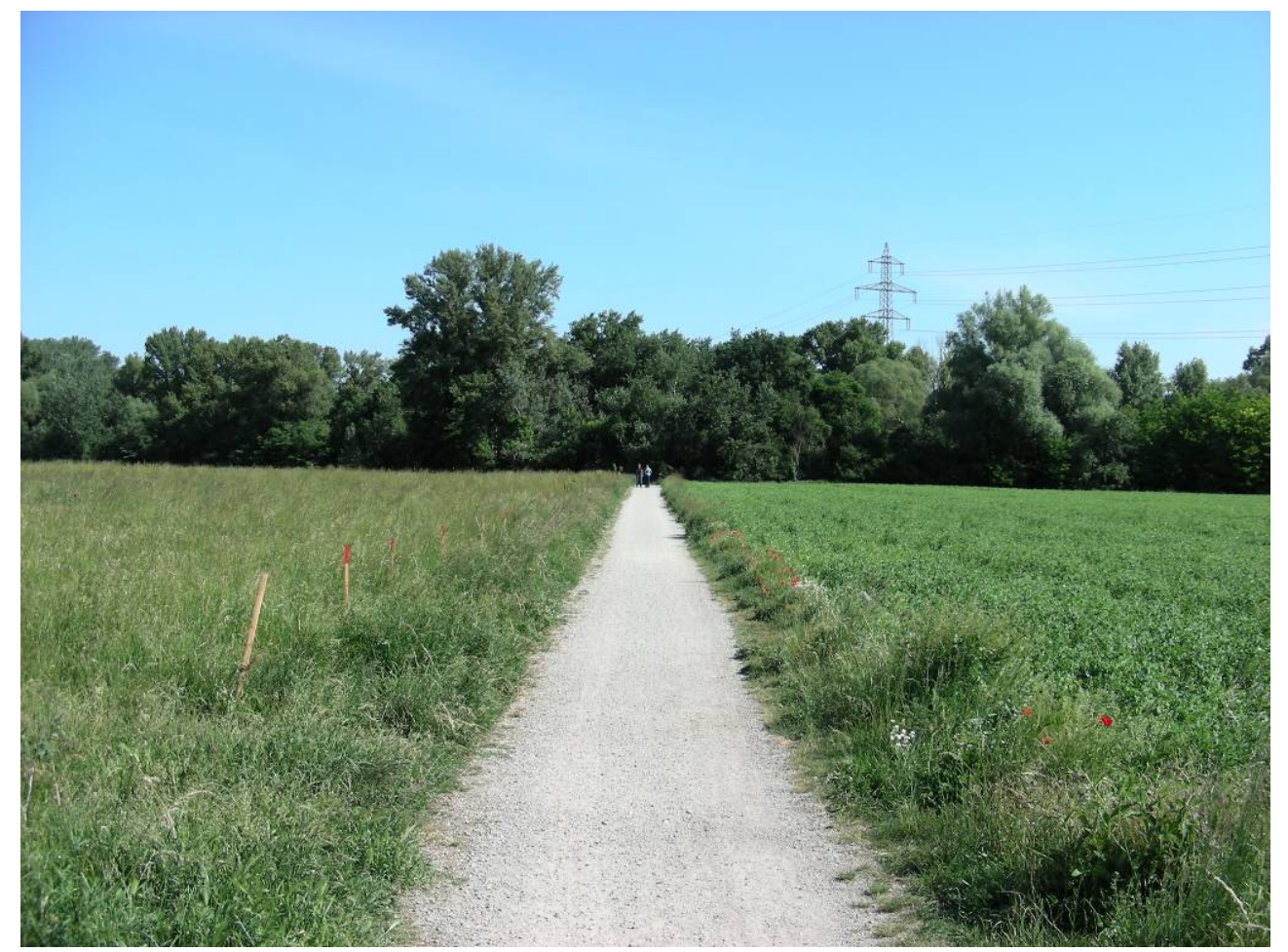

Abb. 12: Ausgewählter Weg für das Wahlmodell 


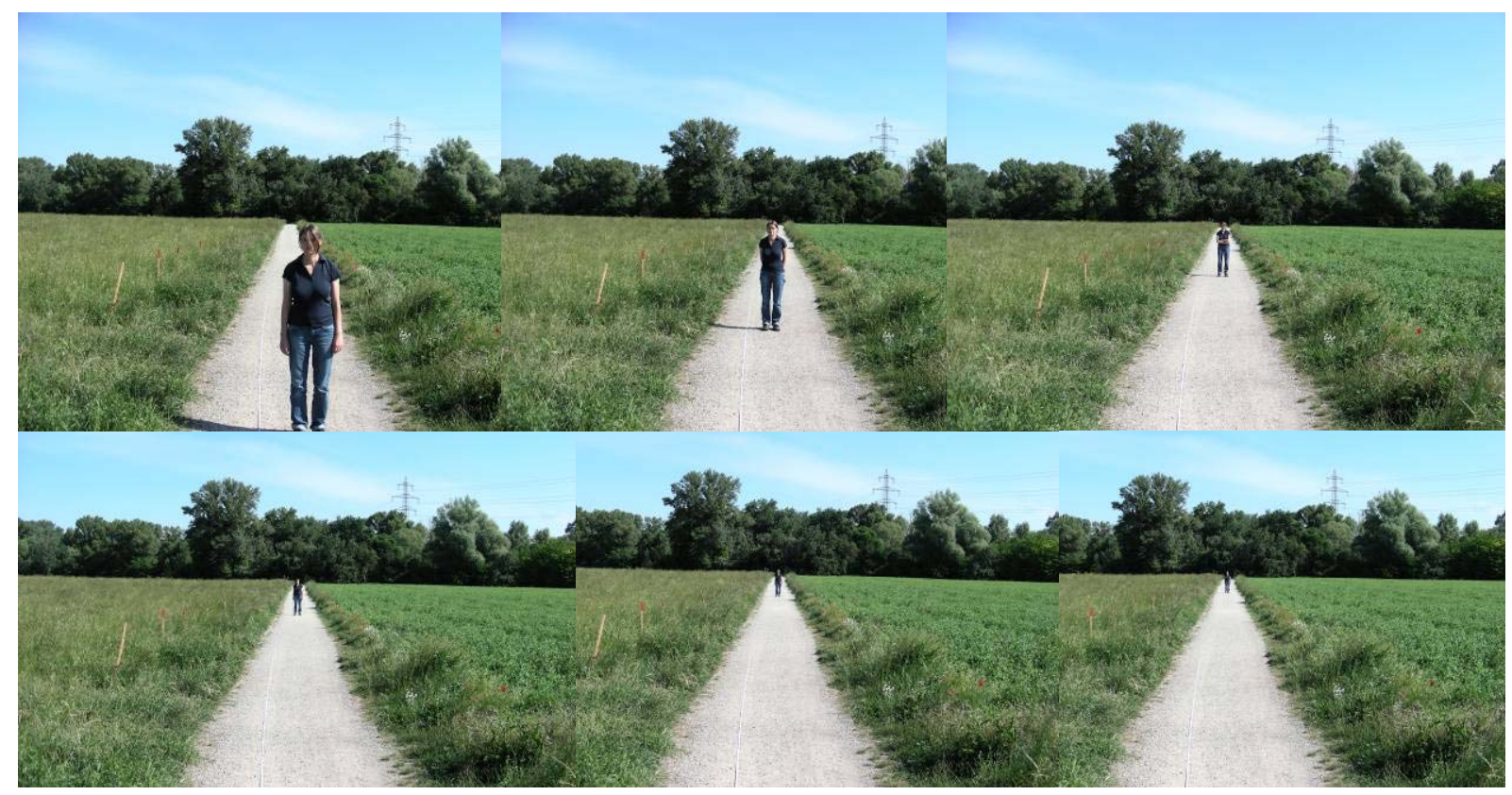

Abb. 13: Fotoserien zur Größenskalierung der Personen in den Bildern

Ein Szenario bestand aus je einem Wegbild, in welches neun Attribute eingearbeitet waren, einem Gewässerbild und ein bis zwei textlichen Attributen (Abb. 14 und Abb. 15). Jeweils vier Szenarien (=Wahlset) wurden auf einem A3 Blatt arrangiert. Jede/r Befragte bekam vier Wahlsets vorgelegt und sollte dabei jeweils die für sie/ihn am meisten und die am wenigsten bevorzugte Wegesituation auswählen (=Wegepräferenzen). Die Befragten konnten auch angeben, auf welchen Wegabschnitten sie überhaupt nicht gehen würden.

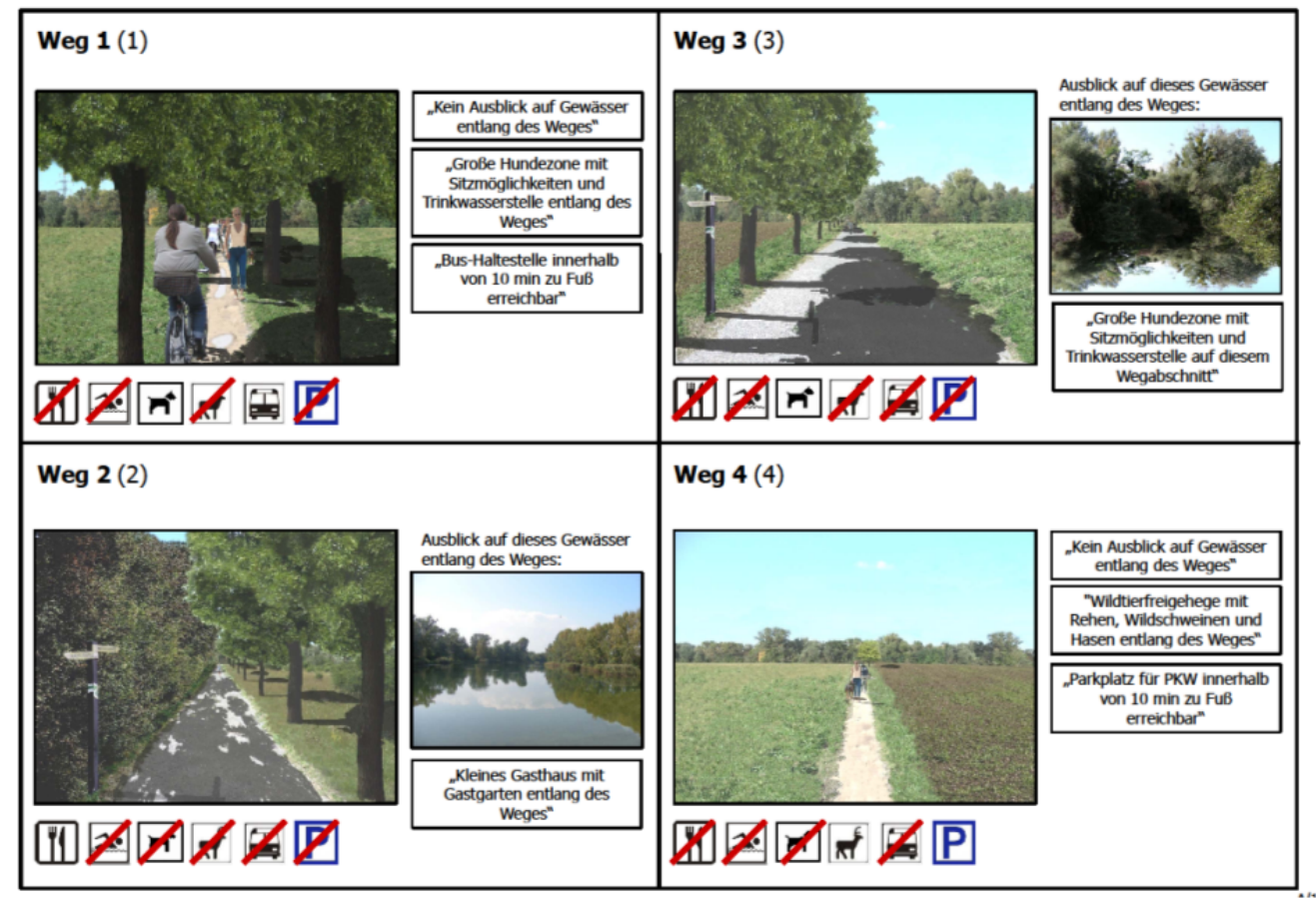

Abb. 14: Beispiel für ein Wahlset mit vier Szenarien aus dem Wahlmodell 


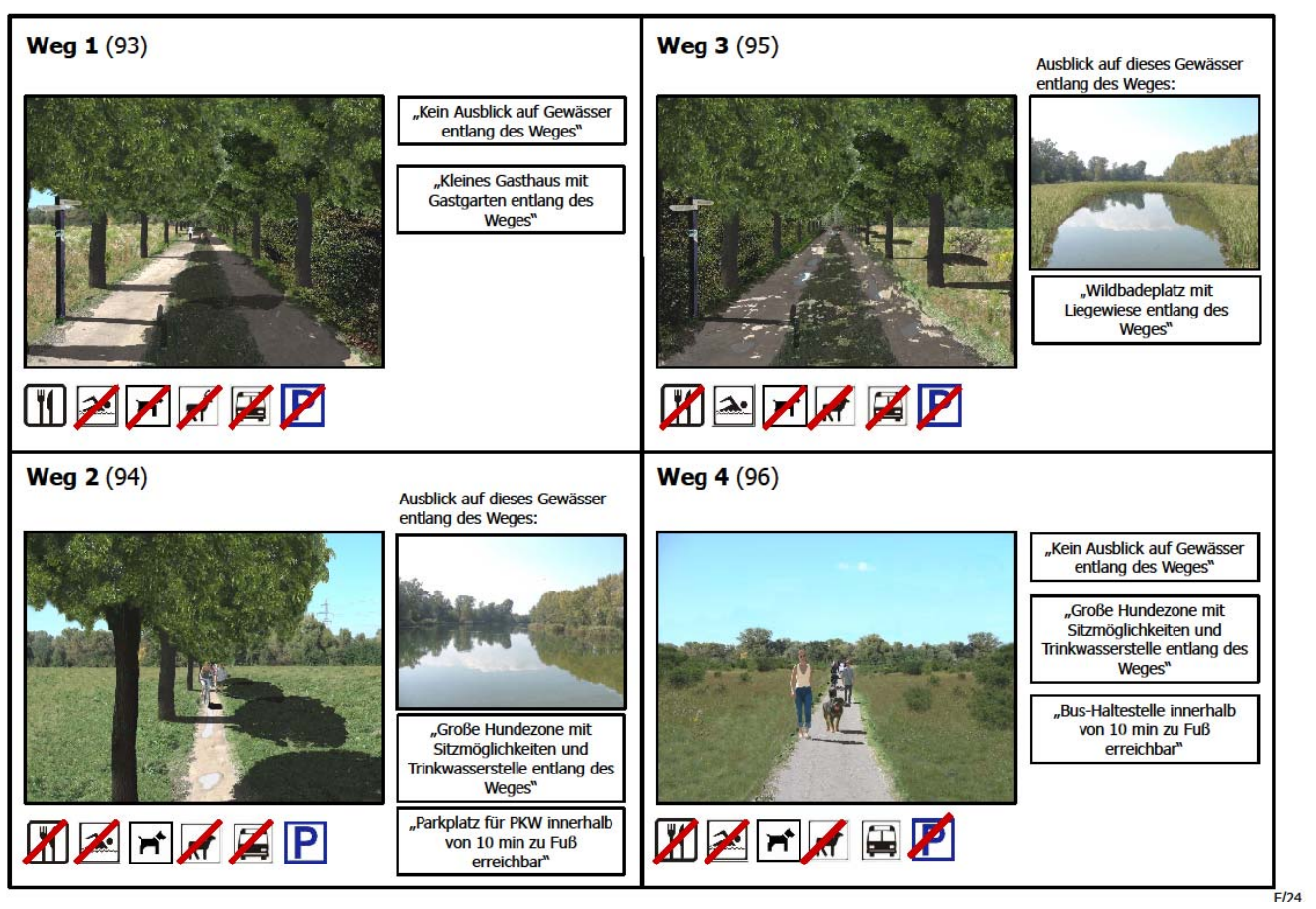

Abb. 15: Beispiel für ein Wahlset mit vier Szenarien aus dem Wahlmodell

Der Fragebogen enthielt neben den Fragen zu den Landschaftspräferenzen noch folgende Fragen bzw. sozialpsychologische Konzepte:

- Profil der Befragten

- $\quad$ Aktivitäten (v.a. Baden, Radfahren und Hund ausführen)

- Erfahrungsgrad mit dem Gebiet und den ausgeübten Aktivitäten

- Wahrnehmung der Besuchermenge und weitere Indikatoren zur Qualität des Erholungserlebnisses

- Gebietseignung für Freizeitaktivitäten

- Ortsbindung

\subsubsection{Befragung des Wahlmodells}

Im zweiten Projektjahr wurden im Zeitraum von Juli 2009 bis September 2009549 Personen bzgl. ihrer Erholungspräferenzen befragt. Die Befragungen wurden an neun Erhebungstagen (5 Werktage und 4 Sonntage) als persönliche Interviews mittels standardisiertem Fragebogen an den fünf LobauEingängen - Dechantweg, Saltenstraße, Eßlinger Furt, Uferhaus und Mühlleiten - durchgeführt. Die Interviews erfolgten den gesamten Tag. Die Verweigerungsrate lag über alle NutzerInnengruppen hinweg bei $57 \%$, wobei vor allem die JoggerInnen mit $71 \%$ eine hohe Verweigerungsrate aufwiesen.

Sowohl Präferenzen hinsichtlich der dargestellten Wegeszenarien, als auch die Bereitschaft diese Wege für bestimmte Freizeitaktivitäten wie Rad fahren oder Hund ausführen zu benutzen, wurden erfasst. Damit wurde die relative Wichtigkeit der in den Bildern dargestellten Attribute im Hinblick auf die einzelnen Aktivitäten bestimmt. Die Daten der Befragung wurden in einer Access-Datenbank erfasst und mit den Programmen SPSS und Latent Gold Choice 4 (Vermunt \& Magidson, 2003) ausgewertet. 


\subsubsection{Resultate der Befragung}

\subsubsection{Struktur der Befragten}

Der Anteil von befragten Männern war mit $64 \%$ etwas höher als jener von Frauen, das Durchschnittsalter lag bei 52 Jahren. $82 \%$ der befragten BesucherInnen wohnten in Wien, davon stammten $60 \%$ aus Wien XXII. $13 \%$ kamen aus der Gemeinde Groß-Enzersdorf (Abb. 16 und Abb. 17). Nur 9 Befragte waren aus Ausland. Knapp die Hälfte nutzte das Rad zur Anreise (49\%), $38 \%$ den PKW, $10 \%$ kamen zu Fuß und $4 \%$ nutzten den ÖV. Die meisten der Befragten waren mit dem Rad unterwegs (53\%). Weitere Nutzergruppen waren FußgängerInnen ohne Hund $(22 \%)$, FußgängerInnen mit Hund (12\%), JoggerInnen (6\%) und Nordic WalkerInnen (5\%).

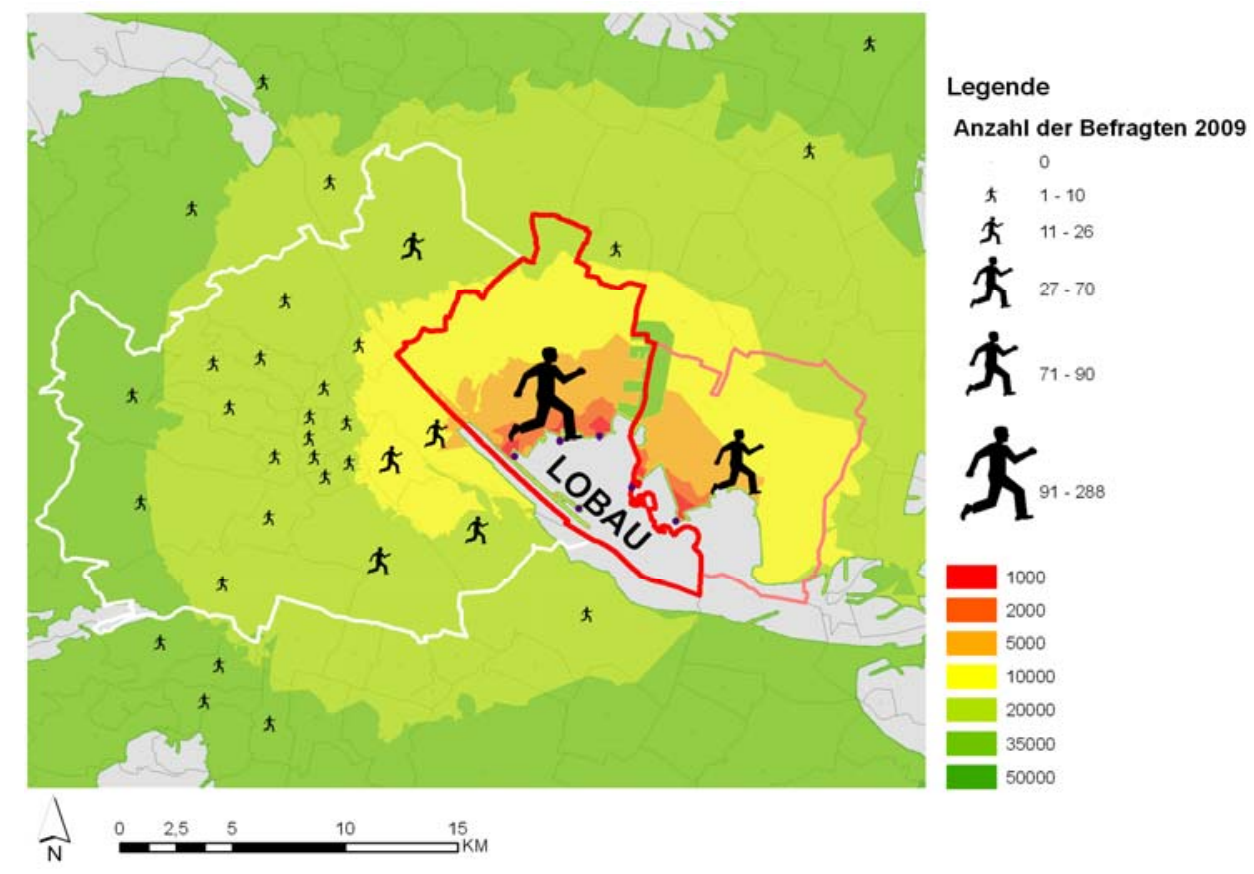

Abb. 16: Anzahl der Befragten pro Bezirk/Gemeinde

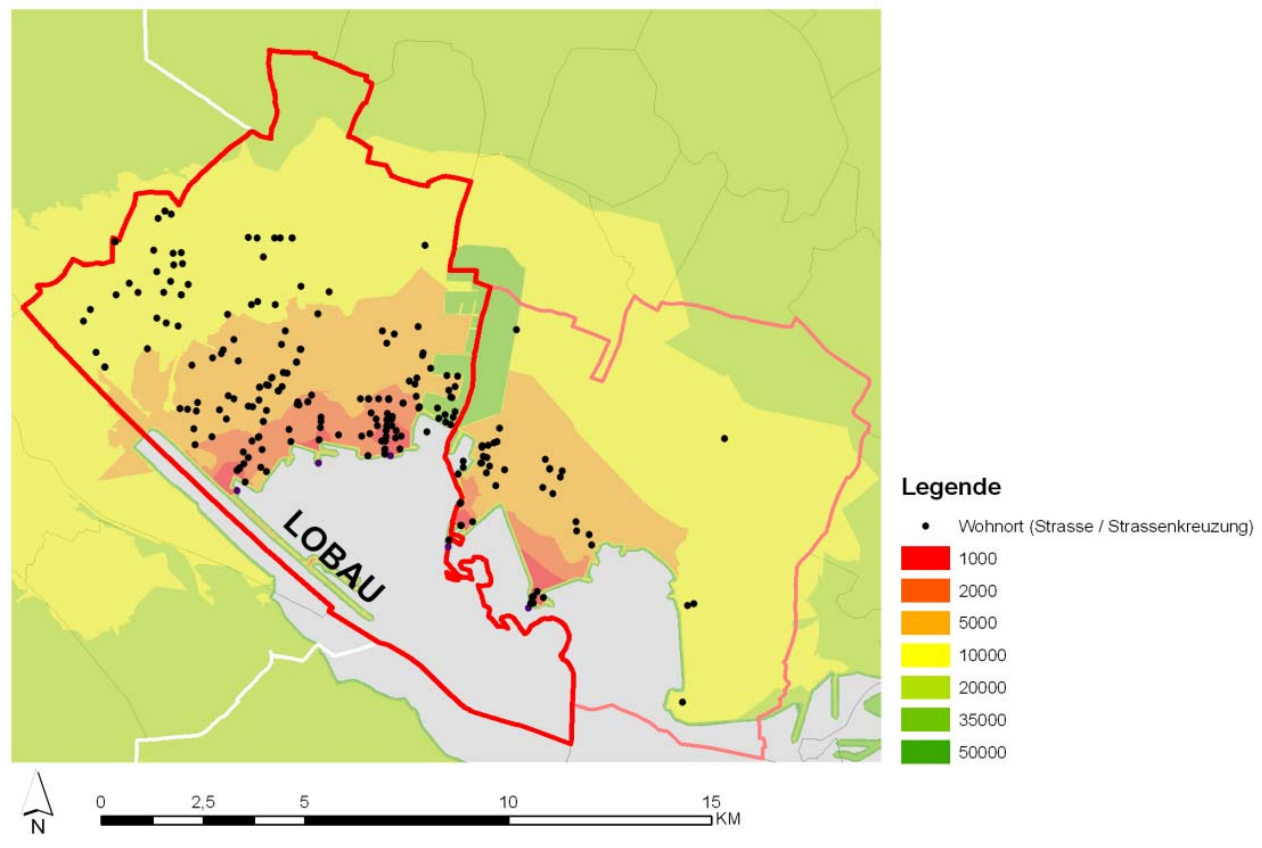

Abb. 17: Lage der Wohnorte der Befragten im 22. Bezirk und der Gemeinde Groß-Enzersdorf 
Um auf den Modal Split der künftigen EinwohnerInnen (z.B. Seestadt Aspern) rückschliessen zu können, wurden jene BesucherInnen, die zwischen 2 und $4 \mathrm{~km}$ von den Wiener Eingängen entfernt wohnten, hinsichtlich ihrer Anreisemittelwahl genauer analysiert. Befragte, die aus diesem Wohnbereich stammten ( 2 bis $4 \mathrm{~km}$ von den Eingängen Saltenstraße oder Esslingerfurt), kamen mit dem PKW oder mit dem Fahrrad zur Lobau (Abb. 18 bis Abb. 21).
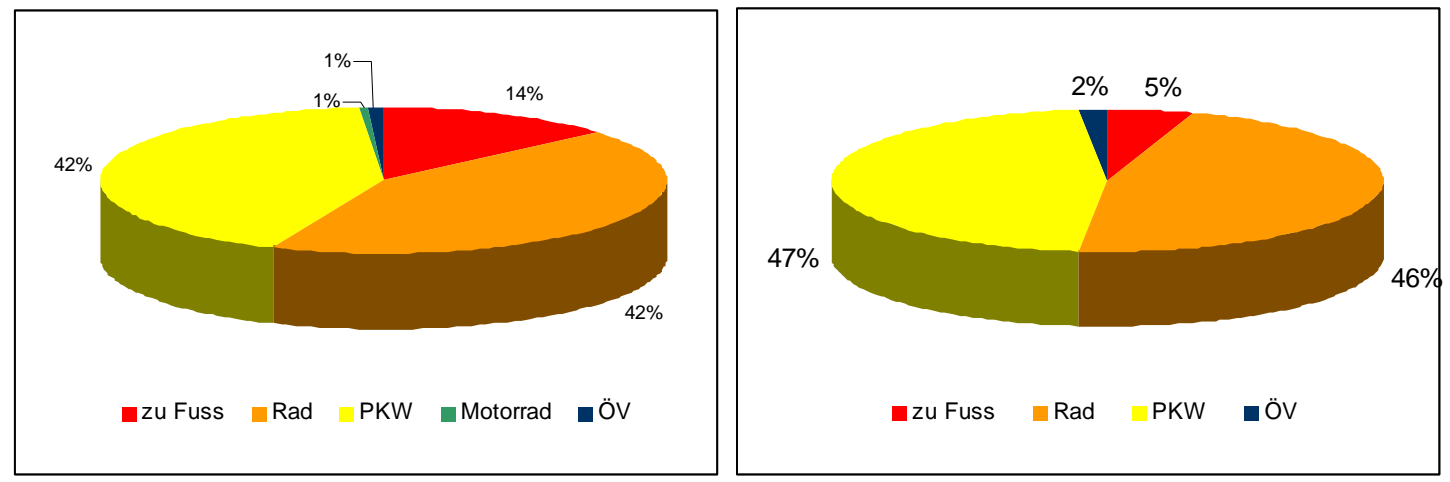

a) alle Wohnorte $(\mathrm{N}=140)$

b) Wohnorte in der Entfernungszone 2-4 km ( $\mathrm{N}=60)$

Abb. 18: Verteilung der Befragten nach Anreisearten (Eingänge Saltenstrasse und Esslingerfurt)
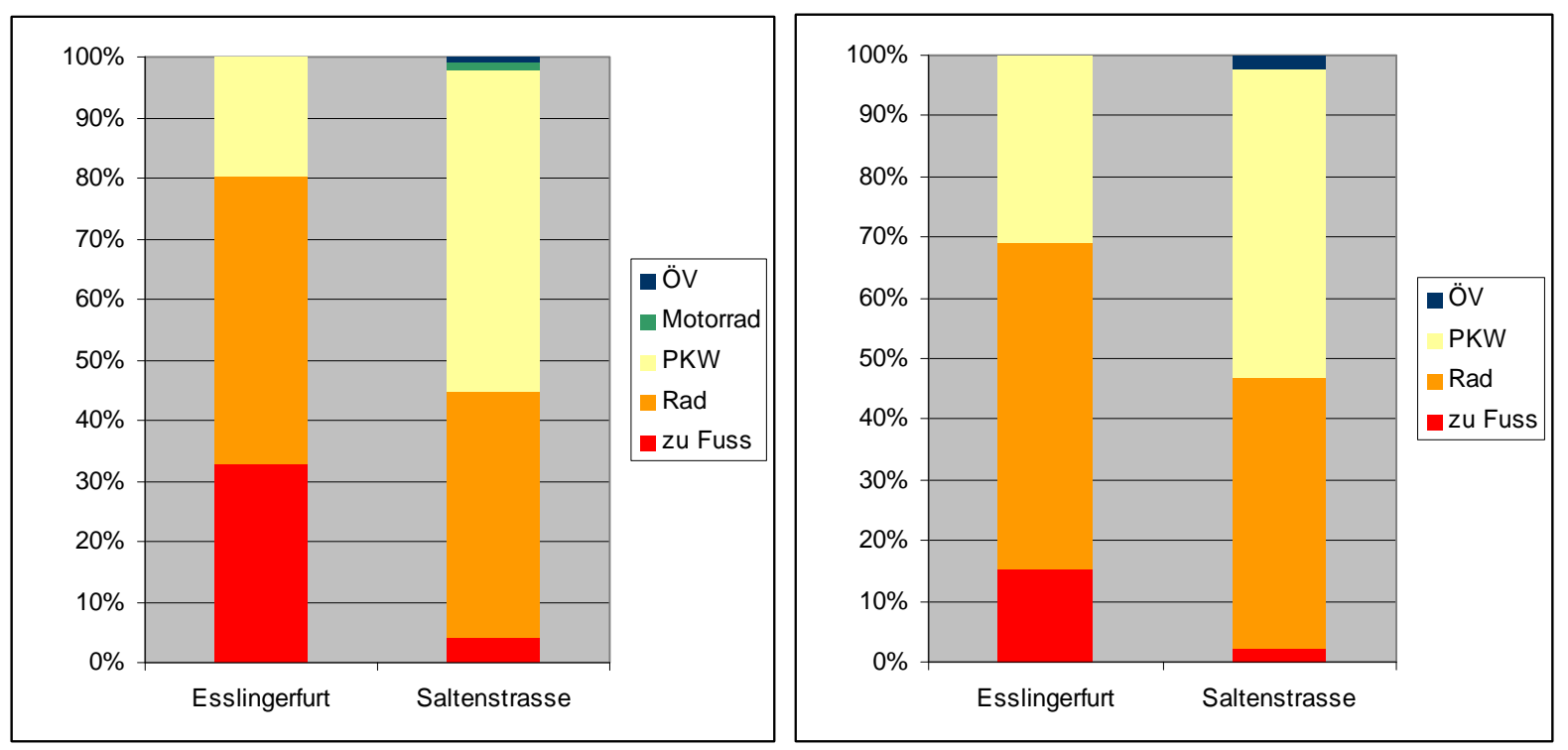

a) alle Wohnorte $(\mathrm{N}=140)$

b) Wohnorte in der Entfernungszone $2-4 \mathrm{~km}(\mathrm{~N}=60)$

Abb. 19: Verteilung der Befragten nach Anreisearten - Vergleich der Eingänge Saltenstrasse und Esslingerfurt 


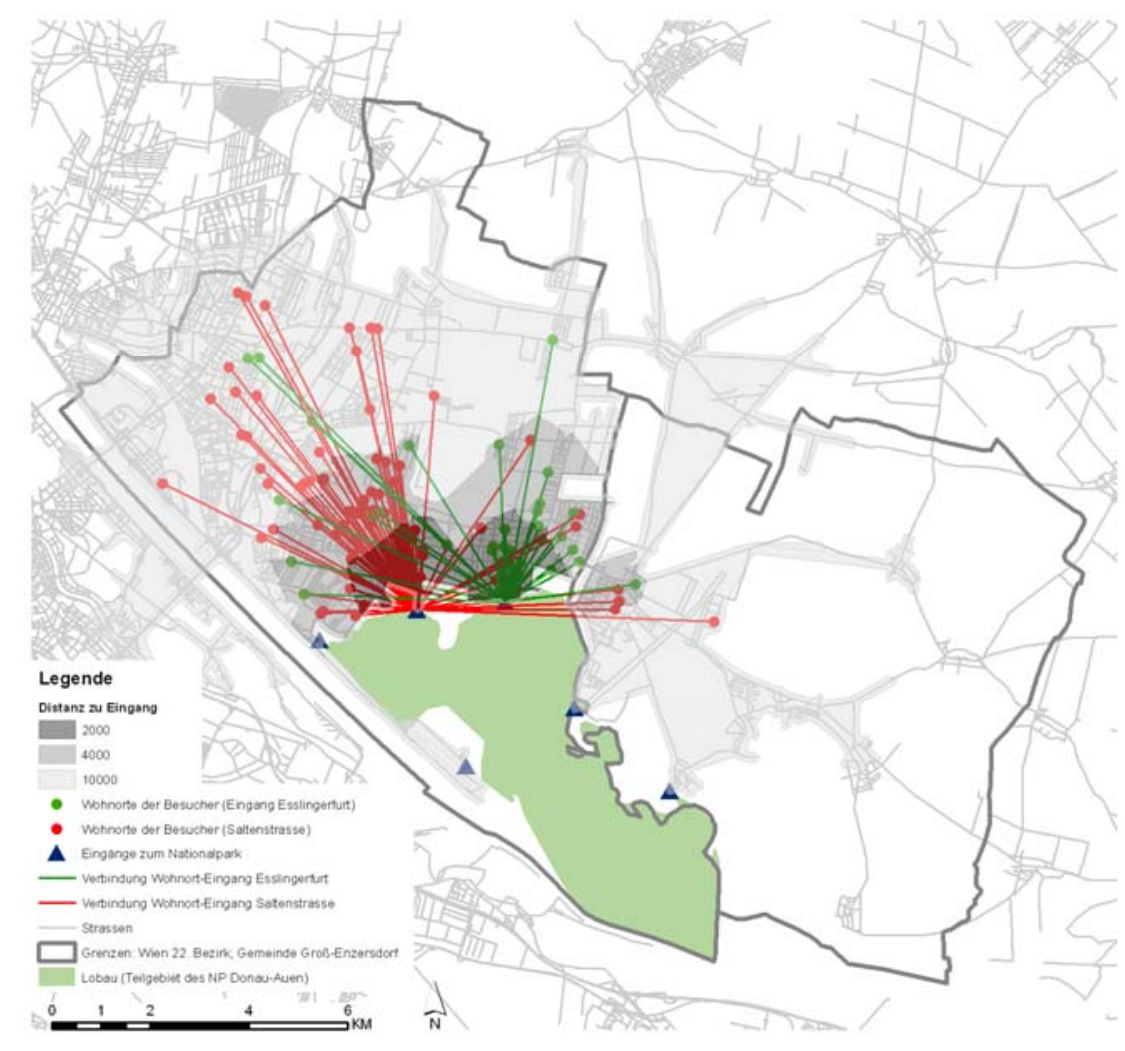

Abb. 20: Erreichbarkeit der NP-Eingänge Saltenstrasse und Esslingerfurt (Graustufen). Darstellung der Quell-Ziel-Beziehungen (Wohnort-Eingang) für die Eingänge Saltenstrasse und Esslingerfurt

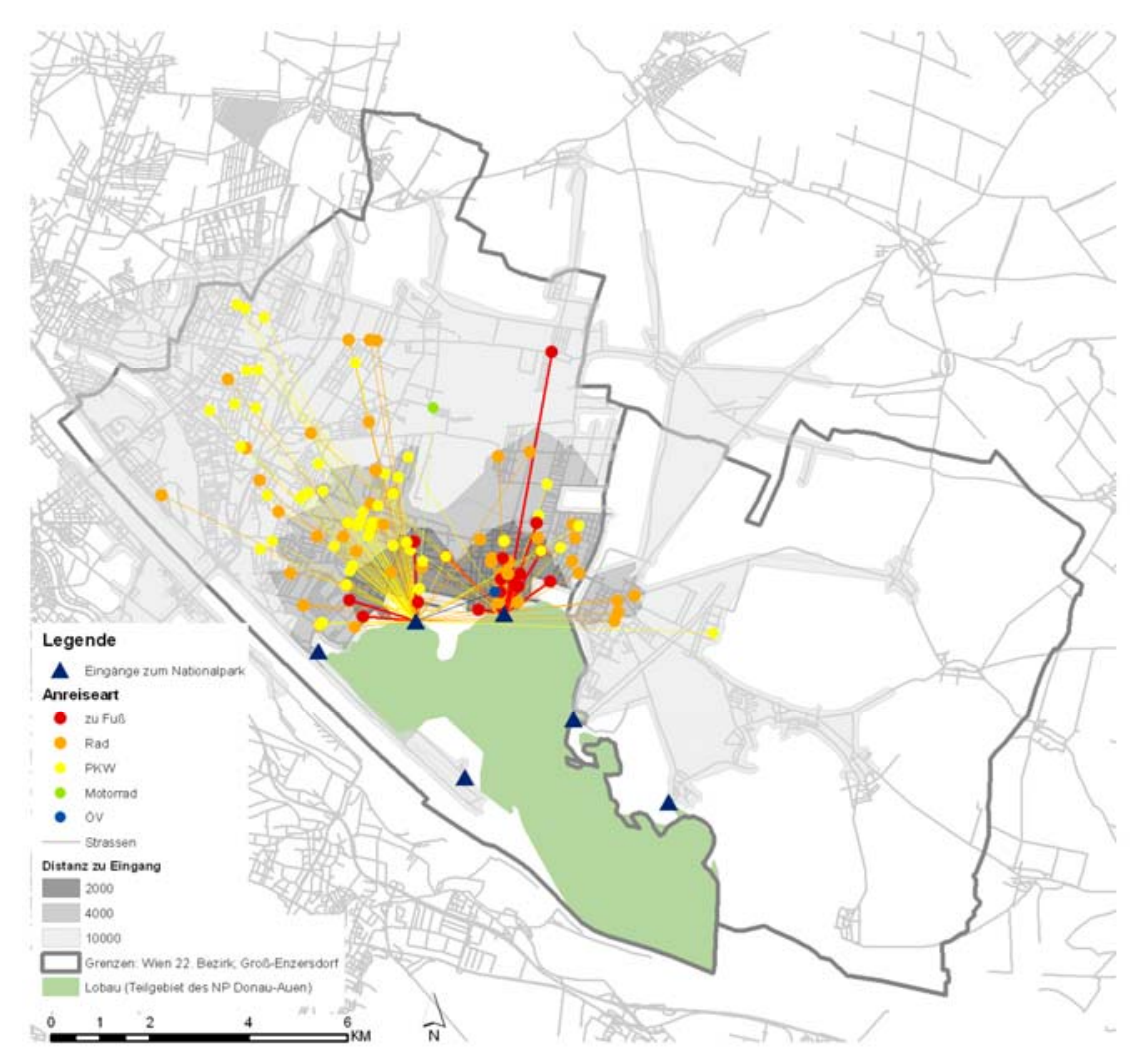

Abb. 21: Erreichbarkeit der NP-Eingänge Saltenstrasse und Esslingerfurt (Graustufen). Darstellung der Quell-Ziel-Beziehungen (Wohnort-Eingang) gruppiert nach Anreiseart 


\subsubsection{Besuchshäufigkeit und-zufriedenheit}

In den zurückliegenden zwölf Monaten hatten die Befragten die Lobau durchschnittlich 88-mal besucht. $30 \%$ der Befragten empfanden die Besuchermenge in der Lobau an den Sonntagen zu hoch, während jene an den Wochentagen als angenehm eingestuft wurde. Die BesucherInnen bewerteten sowohl die Lobau selbst, als auch das Besuchserlebnis in der Lobau als sehr positiv: Für den Zufriedenheitsgrad mit der Lobau ergab sich die Note 1,4 (auf einer Skala von 1 bis 5), für das Besuchserlebnis 1,8 (auf einer Skala von 1 bis 10), wobei 1 ein ausgezeichnetes Besuchserlebnis anzeigt.

\subsubsection{Aktivitäten der Befragten}

Die von den Befragten am häufigsten ausgeübten Aktivitäten in der Lobau in den zurückliegenden zwölf Monaten vor der Befragung waren Radfahren, Wandern und den Hund ausführen (Tab. 11).

\begin{tabular}{|l|c|}
\hline \multicolumn{1}{|c|}{ Aktivitäten } & Mittelwerte \\
\hline Radfahren & 32,9 \\
\hline Wandern & 28,4 \\
\hline Hund ausführen & 25,6 \\
\hline Joggen & 9,7 \\
\hline Nordic Walken & 7,2 \\
\hline Mountainbiken & 3,3 \\
\hline Baden & 5,2 \\
\hline Sonstige & 1,4 \\
\hline
\end{tabular}

Tab. 11: Häufigkeit der ausgeübten Aktivitäten in der Lobau.

\subsubsection{Auswertungen zu den Präferenzen der Erholungssuchenden auf Basis des Wahlmodells}

Alle elf der dargestellten Attribute waren signifikant. Die umgebende Landschaft (Heißlände, Wald, Acker, Wiese,...) und die Personenanzahl (1 bis 16 Personen) am Bild hatten mit $17 \%$ den größten Einfluss auf die Entscheidung. Heißländen und die Kombination von Wiese und Wald wurden von den Befragten am meisten präferiert. Abgelehnt wurden Äcker und auch die Kombination von Äcker und Wiese (Abb. 22). Positiv beurteilt wurden eine Person und vier Personen am Weg, negativ hingegen Gruppen mit acht oder 16 Personen (Abb. 23).

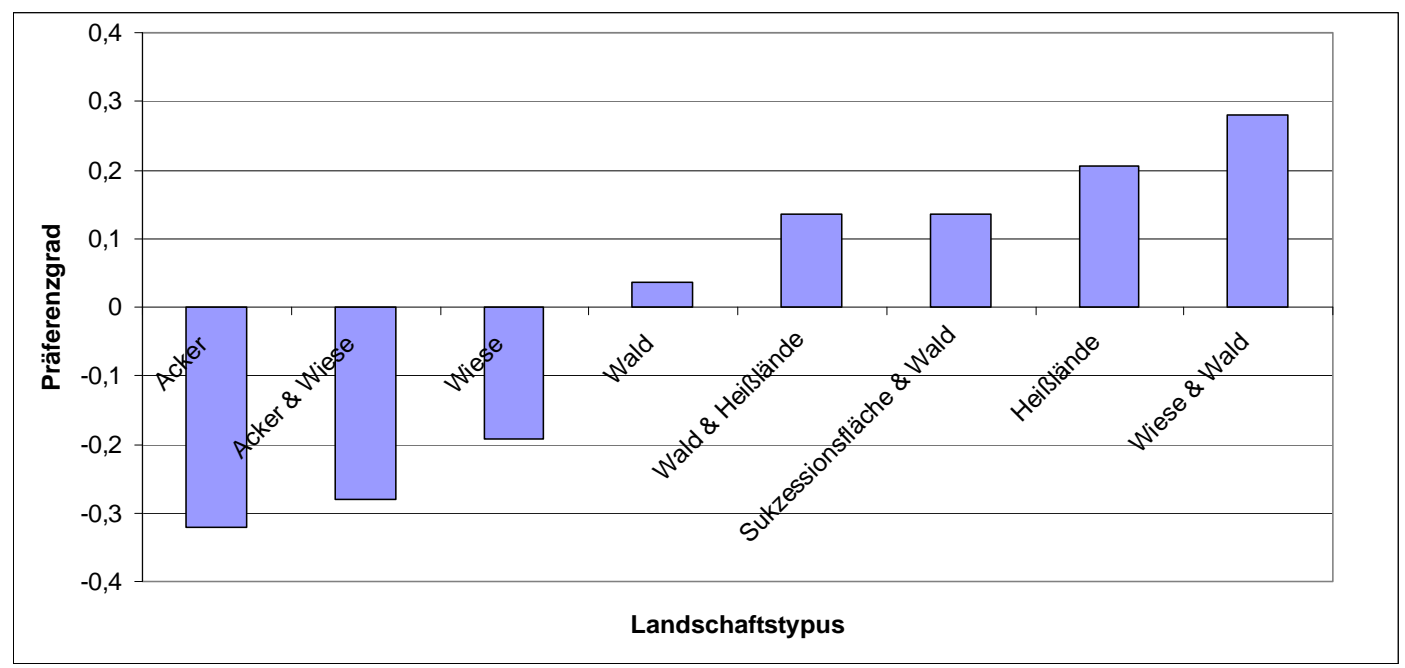

Abb. 22: Präferenzgrad für die im Wahlmodell dargestellten Landschaftstypen ( $N=520)$. Je positiver der Wert, desto höher der Präferenzgrad. 


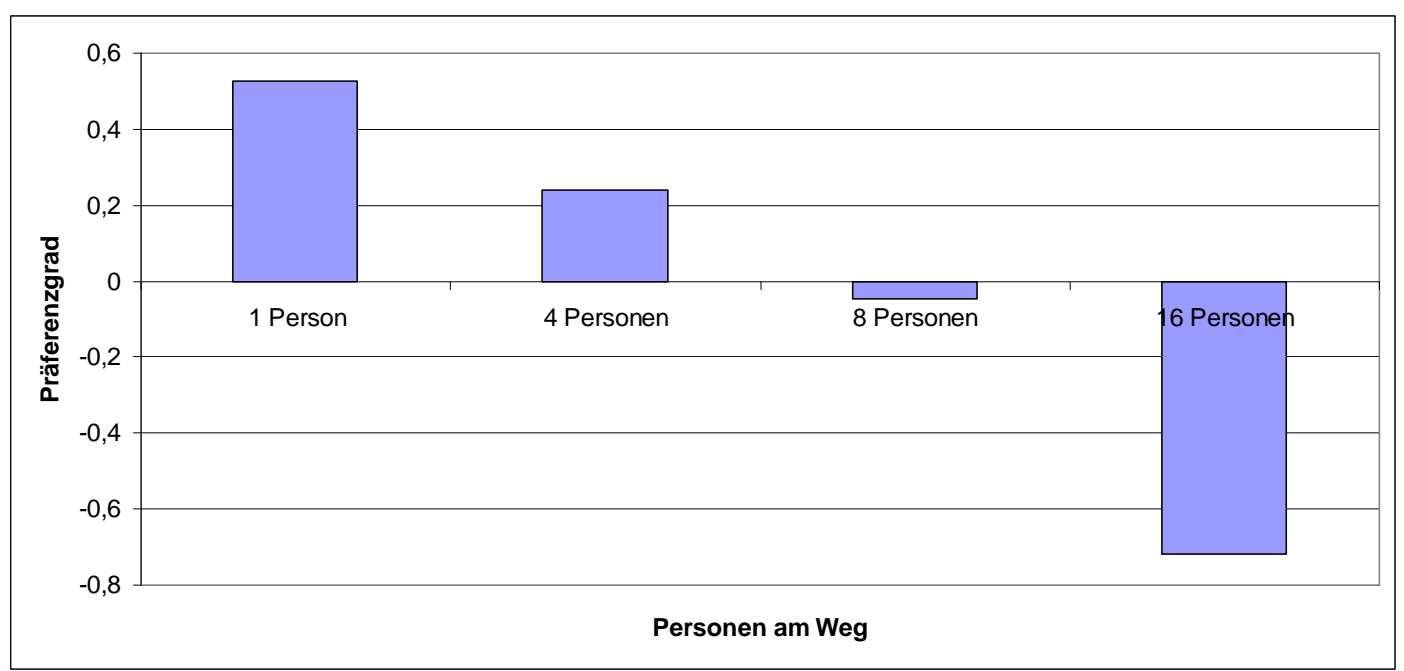

Abb. 23: Präferenzgrad für die im Wahlmodell dargestellte Besucheranzahl $(\mathrm{N}=520)$. Je positiver der Wert, desto höher der Präferenzgrad.

Auch die Art des Gewässers mit $16 \%$, die Gestaltung des Horizontes (Waldkulisse mit oder ohne Strommast, Siedlung) mit $15 \%$ und das Wegbegeleitgrün mit $14 \%$ waren wichtige Faktoren bei der Entscheidung der Befragten. Besonders negativ beurteilt wurden Wege, die keinen Ausblick auf Gewässer boten. Die Befragten präferierten sowohl große, als auch kleine Altarme mit $50 \%$ Schilfbedeckung. Ebenfalls gewünscht waren große Altarme ohne Schilfbedeckung (Abb. 24). Das Angebot an Erholungsinfrastruktur beeinflusste mit $6 \%$ die Entscheidung. Die NutzerInnenzusammensetzung (FußgängerInnen, RadfahrerInnen) und der Anteil an mitgeführten Hunden (mit und ohne Leine) fielen mit $3 \%$ etwas weniger ins Gewicht.

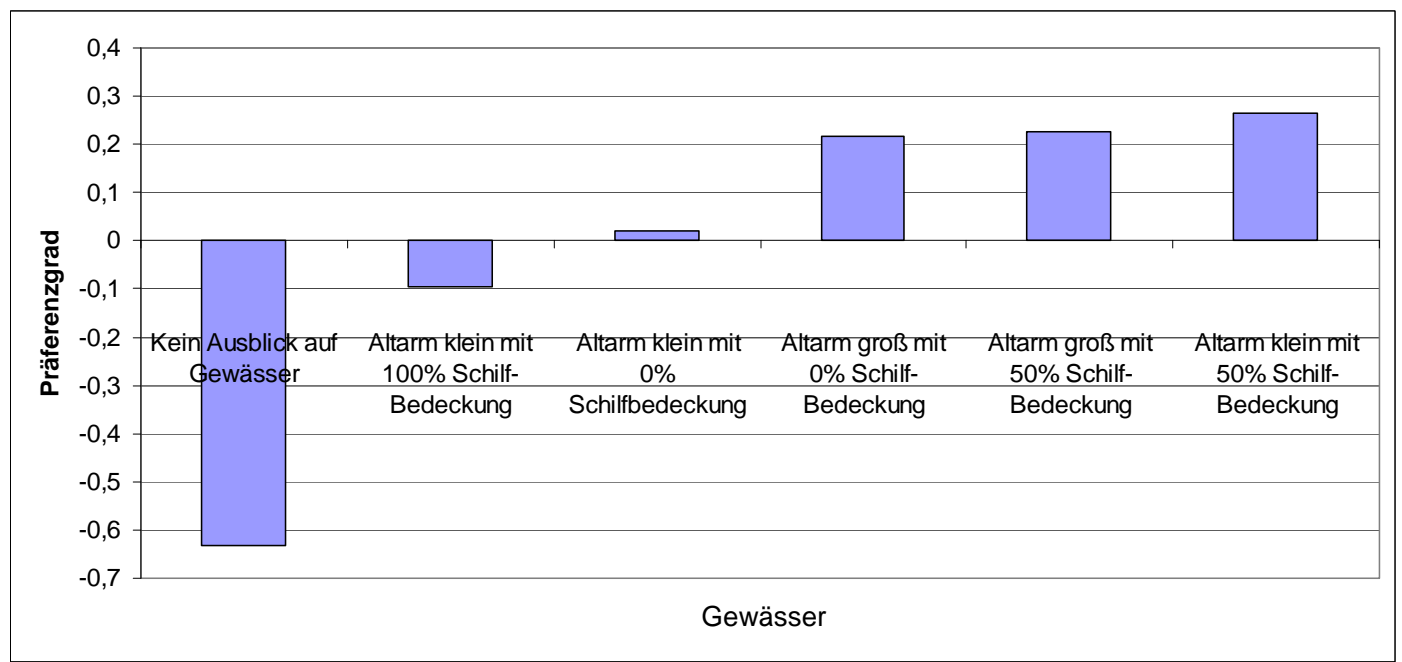

Abb. 24: Präferenzgrad für den im Wahlmodell dargestellten Gewässertypus ( $N=520)$. Je positiver der Wert, desto höher der Präferenzgrad.

Die Relevanz bestimmter Erholungsinfrastruktureinrichtungen oder Landschaftselemente hinsichtlich der Wegewahl der Befragten war auch von der Freizeitaktivität abhängig (Tab. 12). Für die Aktivität Radfahren sind alle Variablen von Bedeutung. Als besonders wichtig wurden die Personenanzahl auf dem Weg und die Wegbreite angesehen. Im Gegensatz dazu sind für die Hundeausführenden nur die drei Attribute - Landschaft, Personenanzahl und Hintergrundkulisse - von Relevanz. Über alle Aktivitäten hinweg gesehen, weist die Anzahl der Personen auf dem Weg die höchste relative Wichtigkeit auf. 


\begin{tabular}{|l|r|r|r|r|r|}
\hline \multicolumn{1}{|c|}{ Attribute } & Radfahren & $\begin{array}{c}\text { Spazieren } \\
\text { gehen }\end{array}$ & $\begin{array}{c}\text { Nordic } \\
\text { Walken }\end{array}$ & $\begin{array}{c}\text { Hund } \\
\text { ausführen }\end{array}$ & Joggen \\
\hline Landschaft & $11 \%$ & $14 \%$ & $14 \%$ & $22 \%$ & $23 \%$ \\
\hline Personenanzahl & $26 \%$ & $26 \%$ & $28 \%$ & $27 \%$ & $13 \%$ \\
\hline Gewässer & $5 \%$ & $12 \%$ & $10 \%$ & & $13 \%$ \\
\hline Hintergrundkulisse & $11 \%$ & $14 \%$ & $15 \%$ & & \\
\hline Wegbegleitgrün: & $5 \%$ & $9 \%$ & $9 \%$ & & $7 \%$ \\
\hline Erholungsinfrastruktur & $8 \%$ & $9 \%$ & & & $7 \%$ \\
\hline Wegebelag & $5 \%$ & $6 \%$ & & & \\
\hline Wegebreite & $16 \%$ & & & & \\
\hline Nutzertyp & $4 \%$ & & & & \\
\hline Hunde & $4 \%$ & & & & \\
\hline Anreise & $4 \%$ & & & \\
\hline
\end{tabular}

Tab. 12: Relative Wichtigkeit der dargestellten Attribute auf die einzelnen Aktivitäten $(N=520)$

\subsubsection{Heterogenität hinsichtlich Landschaftspräferenzen}

Um einer möglichen Heterogenität der Befragten hinsichtlich ihrer Landschaftspräferenzen Rechnung tragen zu können, wurden die Wahlentscheidungen nach latenten Klassen untersucht. Diese Methode erlaubt das Identifizieren und Quantifizieren von Klassen innerhalb der Befragten aufgrund ihrer mehrfachen Wahlentscheidungen (Kemperman \& Timmermans, 2006). Latent-class Wahlmodelle stellen somit eine Erweiterung der diskreten Wahlmodelle wie auch der mixed-logit Modelle dar. Latent-class Modelle wurden bisher in verschiedensten Bereichen eingesetzt, z.B. Transportwesen (Hensher et al., 2005) oder Tourismus und Erholung (Kemperman \& Timmermans, 2006; Scarpa \& Thiene, 2005). Anwendungen im Bereich städtischer Erholungsgebiete finden sich bei Arnberger und Eder (2009), Arnberger et al. (2010) oder Reichhart und Arnberger (2010). Das derzeit wohl am häufigsten eingesetzte Kriterium zur Bestimmung der richtigen Anzahl an Klassen ist das Bayesian Information Criterion (BIC). Je niedriger der Wert, desto besser die Klassenlösung. Die Schätzung erfolgte mit dem Programm Latent Gold Choice 4.0 (Vermunt \& Magidson, 2003). Ein Signifikanzniveau von $\mathrm{p}<.05$ wurde festgelegt.

Nach latenten Klassen wurde hinsichtlich der Eignung für verschiedene Aktivitäten gesucht. Allerdings zeigte sich nur bei der Aktivität Radfahren eine Heterogenität der Befragten. Hier ergaben sich zwei Klassen von nahezu gleicher Segmentgröße. Segment 1 präferiert eher Offenland, mittelbreite Wege, ein paar Menschen am Weg, Hunde, kein Gasthaus und keine Siedlungen am Horizont. Segment 2 bevorzugt eine Wald-Wiesen-Kombination, breite Wege, keine Menschen und Hunde am Weg und das Vorhandensein eines Gasthauses. 


\subsection{Eingabeparameter zur Modellierung der räumlichen Verteilung der BesucherInnen in der Lobau pro Szenario}

Anhand der MASOOR-Simulationssoftware von ALTERRA (Jochem et al., 2008) wurde die Verteilung der Besucherströme simuliert. Dazu wurde ein Wegenetz- und Landschaftsdatenset für die Lobau erstellt. Die Definition der Agenten basierte auf den in der Befragung erhobenen Wegepräferenzen (s. Kap. 4.1) sowie weiteren, über vorangegangene Erhebungen erfasste Eigenschaften der LobaubesucherInnen (Taczanowska, 2009; Taczanowska et al., 2008a, b).

\subsubsection{Agentenbasierte Modellierung des Besucherverhaltens - MASOOR Plattform}

Die agentenbasierte Modellierung ist eine individuenbasierte Methode der computergestützten Modellbildung und Simulation. Die Methode hat ihre Wurzeln in den diversen Bereichen künstlicher Intelligenz (Wooldridge, 2005). Ein Agent wird als ein System in einer Umgebung z.B. in einem sozialen oder physischen Raum bezeichnet, der in der Lage ist diese Umgebung wahrzunehmen und dort zu agieren (Franklin \& Graesser, 1997). Gimblett et al. (1996) führten diesen Ansatz in den Erholungsplanungsbereich ein und legten die methodischen Grundlagen für die Kopplung von agenten-basierten Modellierungstechniken und Geographischen Informationssystemen fest (Gimblett, 2002). Dieses Konzept wurde erfolgreich in den folgenden Simulationsplattformen umgesetzt: RBSim (Gimblett et al., 2002; Itami, 2002), AlpSim (Cavens et al. 2004; Gloor et al., 2003), MASOOR (Jochem et al., 2008; Jochem \& van der Greft, 2005) und Kvintus (Skov-Petersen, 2009).

In Rahmen des Forschungsprojektes „Perspektive Lobau 2020“ wurde die MASOOR-Plattform (Abb. 25 ) verwendet $($ MASOOR $=$ Multi-agent Simulation of Outdoor Recreation), um das räumliche Verhalten der BesucherInnen in der Lobau unter Berücksichtigung verschiedener Planungs- und Management-Maßnahmen zu simulieren. MASOOR wurde von Alterra und der Wageningen Universität entwickelt (Jochem et al., 2008). Die MASOOR-Plattform befindet sich in der Entwicklungsphase und ist im Moment lediglich für Forschungszwecke erhältlich.

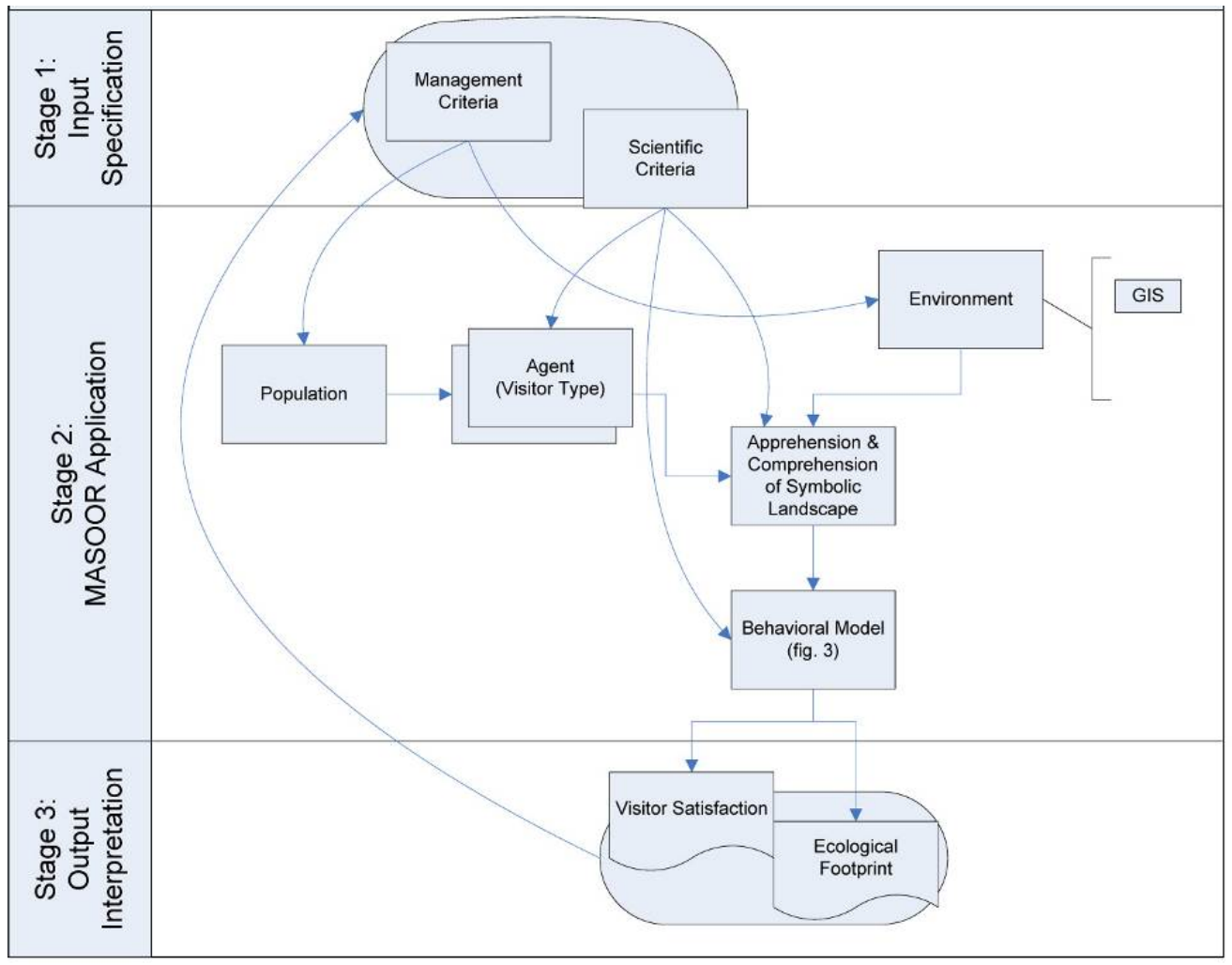

Abb. 25: Komponente eines MASOOR-Models (Jochem et al. 2008) 
Die Software bietet große Flexibilität bei der Festlegung der Eingangsparameter. Dadurch ist eine Anpassung an individuelle Projektziele möglich. Jede MASOOR-Simulation erfolgt in drei Hauptschritten:

- Spezifikation der Eingangsparameter

- Anwendung der MASOOR-Plattform

- Interpretation der Ergebnisse

Im ersten Schritt werden allgemeine Eingangsparameter definiert. In dieser Arbeitsphase geht es vor allem um die Konkretisierung der räumlichen und sozialen Aspekte der Simulationsszenarien:

- Dokumentation bestehender Erholungsinfrastruktur und Landschaftscharakteristika bzw. Festlegung der geplanten Änderungen im Testgebiet (z.B. Verlauf und Eigenschaften von Wegen, Landschaftsbild, Lage der Eingänge)

- Bestimmung relevanter Besuchergruppen und der Determinanten ihres räumlichen Verhaltens

- Festlegung der Besucherzahlen

Die gewünschten Eingangsparameter werden dann in der MASOOR-Applikation formalisiert. Tab. 13 stellt eine Zusammenfassung der Inputdaten eines MASOOR-Models dar.

\begin{tabular}{|c|c|c|c|}
\hline \multicolumn{2}{|c|}{ MASOOR-Eingangsdaten } & \multirow{3}{*}{$\begin{array}{l}\text { Basiselement } \\
\text { Wegabschnitt }\end{array}$} & \multirow{2}{*}{\begin{tabular}{l}
\multicolumn{1}{c}{ Eigenschaften } \\
Topologische \& Geometrische \\
Eigenschaften
\end{tabular}} \\
\hline Räumliche Daten & Wegenetz & & \\
\hline & & & Thematische Eigenschaften \\
\hline & & Kreuzungspunkt & Topologische Eigenschaften \\
\hline & Eingänge & Punkt & Topologische Eigenschaften \\
\hline \multirow{4}{*}{$\begin{array}{l}\text { Soziodemographische und } \\
\text { psychographische Daten }\end{array}$} & \multirow{4}{*}{$\begin{array}{l}\text { Population der } \\
\text { Agenten }\end{array}$} & \multirow[t]{4}{*}{ Agententyp } & Präferenzen (räumlich) \\
\hline & & & Präferenzen (sozial) \\
\hline & & & Aufenthaltsdauer \\
\hline & & & Geschwindigkeit \\
\hline
\end{tabular}

Tab. 13: Inputdaten eines MASOOR-Models

In einem MASOOR Modell sind zwei Haupttypen von Inputdaten zu unterscheiden:

- räumliche Daten

- $\quad$ soziodemographische und psychographische Daten

Die räumlichen Daten werden in einem Geographischen Informationssystem (ArcGIS) als Vektordaten formalisiert. Ein Erholungsgebiet wird anhand des attributisierten Wegenetzes (Shapefile; Polylinie) und der Eingänge (Shapefile; Punkt) abgebildet. In den Wegabschnitten eines Wegenetzes können gewünschte Charakteristika des Erholungsraums in Form von thematischen Attributen gespeichert werden (z.B. Wegbelag, -breite, Landschaft etc.). Die Eingangspunkte enthalten keine zusätzlichen thematischen Attribute und dienen lediglich als Start- bzw. Endpunkte der von den Agenten zurückgelegten Routen.

Die Agenten können sich ausschließlich innerhalb des Wegenetzes bewegen. Die Routenwahl erfolgt während der Tour (,en route“ Entscheidungen). Die Entscheidungen werden von den Agenten an den Kreuzungen getroffen. Jede Kreuzungssituation wird von den Agenten anhand der vordefinierten Bewegungskriterien evaluiert und der optimale nachkommende Wegabschnitt wird ausgewählt.

Die Population der virtuellen Erholungssuchenden kann aus verschiedenen Agententypen bestehen. Die Agenten, die zu einem Agententypus gehören, haben gemeinsame räumliche und / oder soziale Präferenzen. Die Wegabschnittpräferenzen eines Agententyps können von zahlreichen Algorithmen beeinflusst werden. Der Modellierer entscheidet über den Einsatz der Algorithmen in vier nachfolgenden Bewegungsphasen eines Agenten. 
Abb. 26 stellt alle Bewegungsphasen dar. Tab. 14 fasst die möglichen Bewegungsdeterminanten zusammen.

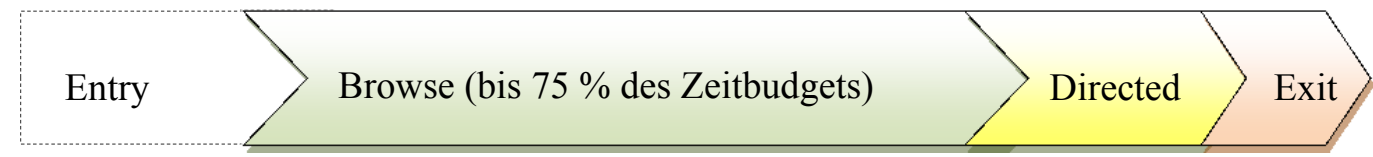

Abb. 26: Bewegungsphasen eines MASOOR-Agenten: 1) Entry Phase - in der Bewegungsphase werden bestimmte Attraktionen / Zielpunkte besucht; diese Phase ist jedoch optional, manche Agententypen fangen den Besuch eines Erholungsgebietes direkt in der zweiten Bewegungsphase an, ohne konkrete Ziele besucht zu haben; 2) Browse Phase Bewegung ohne vordefinierte Ziele, lokale Entscheidungen auf Basis vordefinierter Bewegungskriterien. Die Phase dauert solange, bis $75 \%$ der geplanten Besuchszeit erreicht werden; 3) Directed Phase - der Agent orientiert sich langsam Richtung Ausgang; 4) Exit Phase - die letzte Bewegungsphase (die letzten $10 \%$ der vordefinierten Zeit), die zum Verlassen des Erholungsgebietes führt.

\begin{tabular}{|l|l|}
\hline \multicolumn{1}{|c|}{ MASOOR Algorithmus } & \multicolumn{1}{c|}{ Beschreibung } \\
\hline "Global heading" & Navigation in Richtung gewünschter Destinationen (Attraktionen). \\
\hline "Path type" & $\begin{array}{l}\text { Präferenzen für bestimmte Wegtypen (basierend auf den thematischen } \\
\text { Eigenschaften eines Wegabschnittes, z.B. Wegbelag, Breite etc.). }\end{array}$ \\
\hline "Homing direction" & $\begin{array}{l}\text { Navigation in Richtung Ausgang. Die Wegabschnitte, die Richtung } \\
\text { Ausgangspunkt führen, erhalten höhere Präferenzwerte; besonders wenn } \\
\text { sich die Agenten in der letzten Bewegungsphase (Exit) befinden. }\end{array}$ \\
\hline "Chunking direction" & $\begin{array}{l}\text { Präferenz für jene Wegabschnitte, die eine Kontinuität der momentanen } \\
\text { Bewegungsrichtung ermöglichen. }\end{array}$ \\
\hline "Path segment history" & $\begin{array}{l}\text { Präferenz für jene Wegabschnitte, die während der Route noch nicht } \\
\text { besucht wurden. Dieser Algorithmus wird meist dann verwendet, wenn } \\
\text { Rundwege typisch für das Testgebiet sind. }\end{array}$ \\
\hline "Shortest distance" & $\begin{array}{l}\text { Basisalgorithmus der Navigationsstudien. Der Agent wählt den } \\
\text { Wegabschnitt, der am nächsten zu dem Ziel liegt; meistens wird der } \\
\text { Algorithmus bei der Navigation in Richtung Ausgang verwendet. }\end{array}$ \\
\hline "Crowding" & $\begin{array}{l}\text { Dieses Algorithmus überprüft wie viele andere BesucherInnen (Agenten) } \\
\text { sich momentan auf den angrenzenden Wegabschnitten befinden. Die } \\
\text { Wegabschnitte mit höheren Besucherzahlen erhalten niedrigere } \\
\text { Präferenzwerte. }\end{array}$ \\
\hline
\end{tabular}

Tab. 14: Zusammenfassung der Kriterien, die die Routenwahl (Wegsegmentwahl) eines Agenten beeinflussen können (Jochem et al. 2008)

Darüber hinaus werden die Aufenthaltsdauer sowie die Fortbeweguungsgeschwindigkeiten für jeden Agententypus in der MASOOR-Datenbank festgelegt. Die Definition der Agentenpopulation erfolgt ebenfalls in der MASOOR-Datenbank. In dem Fall wird die Anzahl der Agenten, sowie die prozentuelle Einteilung der Agententypen für jeden Eingang definiert. Anschließend werden alle räumlichen Daten in die MASOOR-Plattform importiert und mit den sozialen Daten integriert. Dabei ist es wichtig, dass die Schlüsselfelder der GIS-Daten mit jenen der MASOOR-Datenbank übereinstimmen. Die Ergebnisse einer MASOOR Simulation liegen in tabellarischer Form vor und können anhand einer ArcGIS-Software kartographisch dargestellt werden.

\subsubsection{Vorbereitung der Eingangsdaten}

Für den MASOOR-Simulationsvorgang wurden 14 verschiedene Varianten ausgewählt (Tab. 15). Die Simulationsvarianten umfassen Szenario „“" (Besuchermenge des Jahres 1999) und die sechs Managementszenarien für das Jahr 2025, jeweils unter Berücksichtigung der Besucherzahlen an einem Spitzen- sowie an einem Werktag. 


\begin{tabular}{|l|l|c|c|}
\hline \multirow{2}{*}{ Nummer } & \multicolumn{1}{|c|}{ Szenario } & \multicolumn{2}{|c|}{ Besucherzahl } \\
\cline { 3 - 4 } & & Jahr & Spitzentag / Werktag \\
\hline 01 & Lobau A & 1999 & Spitzentag \\
\hline 02 & Lobau A & 1999 & Werktag \\
\hline 03 & Lobau A & 2025 & Spitzentag \\
\hline 04 & Lobau A & 2025 & Werktag \\
\hline 05 & Lobau B & 2025 & Spitzentag \\
\hline 06 & Lobau B & 2025 & Werktag \\
\hline 07 & Lobau A + Vorland A & 2025 & Spitzentag \\
\hline 08 & Lobau A + Vorland A & 2025 & Werktag \\
\hline 09 & Lobau A + Vorland B & 2025 & Spitzentag \\
\hline 10 & Lobau A + Vorland B & 2025 & Werktag \\
\hline 11 & Lobau B + Vorland A & 2025 & Spitzentag \\
\hline 12 & Lobau B + Vorland A & 2025 & Werktag \\
\hline 13 & Lobau B + Vorland B & 2025 & Spitzentag \\
\hline 14 & Lobau B + Vorland B & 2025 & Werktag \\
\hline
\end{tabular}

Tab. 15: Aufstellung der simulierten Managementszenarien (Lobau A = Lobau ohne Änderung; Lobau $B=$ Lobau (GewässerSzenario „OPTIMA Lobau 1b“); Vorland A = lineare Ausgestaltung des Lobau-Vorlandes; Vorland B = flächige Ausgestaltung des Lobau-Vorlandes)

Grundlage für die angenommenen Besucherzahlen der zwölf Lobau-Eingänge für das Szenario „,““ war die Erhebungskampagne in der Lobau aus dem Jahr 1999 (Arnberger et al., 2000; Brandenburg, 2001). Eine detaillierte Prognose des Besucheraufkommens für einen Spitzentag sowie einen Werktag im Jahr 2025 wurde im Rahmen dieses Forschungsprojektes erstellt (s. Kap. 2). Die Bestimmung relevanter Besuchergruppen (Agententypen) und den Determinanten ihres räumlichen Verhaltens erfolgte in erster Linie anhand des dargestellten Wahlmodels (s. Kap. 4.1). Die Erfahrungen aus den bisherigen Forschungsarbeiten waren bei der Formalisierung dieser Parameter innerhalb der MASOOR Plattform sehr hilfreich (Taczanowska, 2009; Taczanowska et al. 2008a, b). Sonstige Eingangsdaten wie z.B. Fortbewegungsgeschwindigkeiten, Routenlängen, Dauer des Lobaubesuches stammten aus anderen Studien (Taczanowska et al., 2008b; Arnberger et al., 2009; van Marwijk, 2009).

\subsubsection{Definition der Eingabeparameter}

\subsubsection{Räumliche Daten}

Die räumlichen Daten wurden in einem Geographischen Informationssystem (ArcGIS 9.3) aufbereitet. Im ersten Schritt wurden das Wegenetz (Shapefile; Polylinie) und die Eingänge (Shapefile; Punkt) für die sechs verschiedenen räumlichen Szenarien digitalisiert. Die Anzahl der Wegabschnitte in einem Wegenetz reichte von 306 (Lobau A) bis 349 Abschnitte (Lobau B + Vorland B). Die Gesamtlänge aller Wege lag zwischen 113,8 km (Lobau A) und 125,9 km (Lobau A + Vorland B).

Für die Szenarien Lobau A und Lobau B wurden jeweils zwölf Eingangspunkte definiert (Dechantweg, Luitpold-Stern-Gasse, Saltenstraße, Eßlinger Furt, Stadler Furt, Egerer Brücke, Kasernenbrücke, Uferhaus, Mühlleiten, Hubertusdamm, Panozzalacke, Bahnhof Lobau). Für die Szenarien, die Managementmaßnahmen im Lobau-Vorland vorsahen, wurden elf Eingänge definiert (Dechantweg, Saltenstraße, Eßlinger Furt, Stadler Furt, Egerer Brücke, Kasernenbrücke, Uferhaus, Mühlleiten, Hubertusdamm, Panozzalacke, Bahnhof Lobau). Abb. 27 bis Abb. 29 veranschaulichen die sechs Varianten des Wegenetzes und die dazugehörigen Eingänge. 


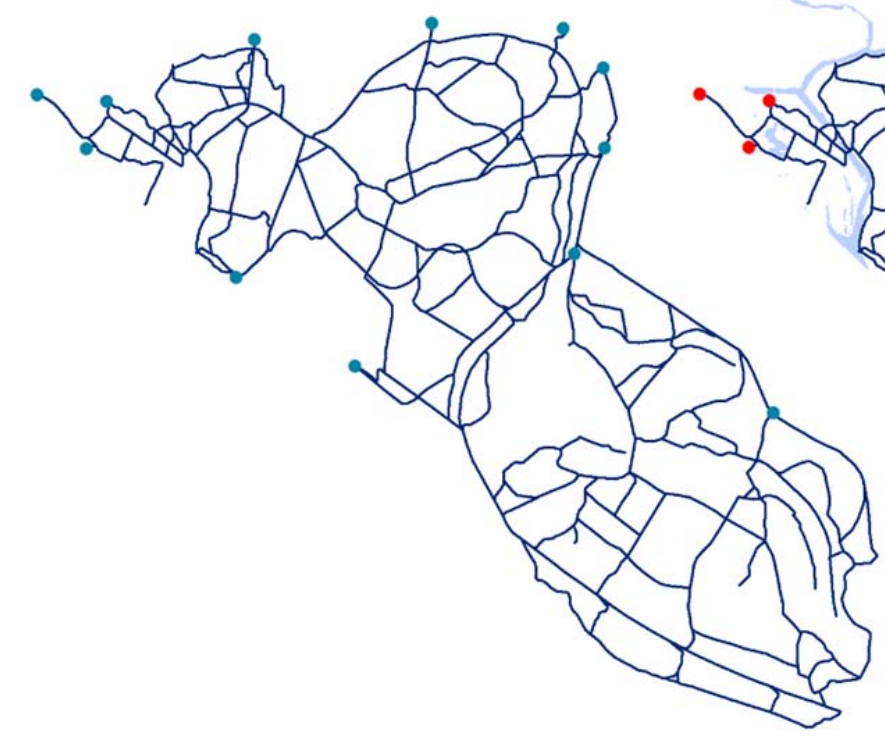

A)

Abb. 27: Inputdaten für die Simulation der Besucherströme in der Lobau. A) Wegenetz: Variante „Lobau A“ und 12 Eingänge; B) Wegenetz: Variante „Lobau B“ und 12 Eingänge

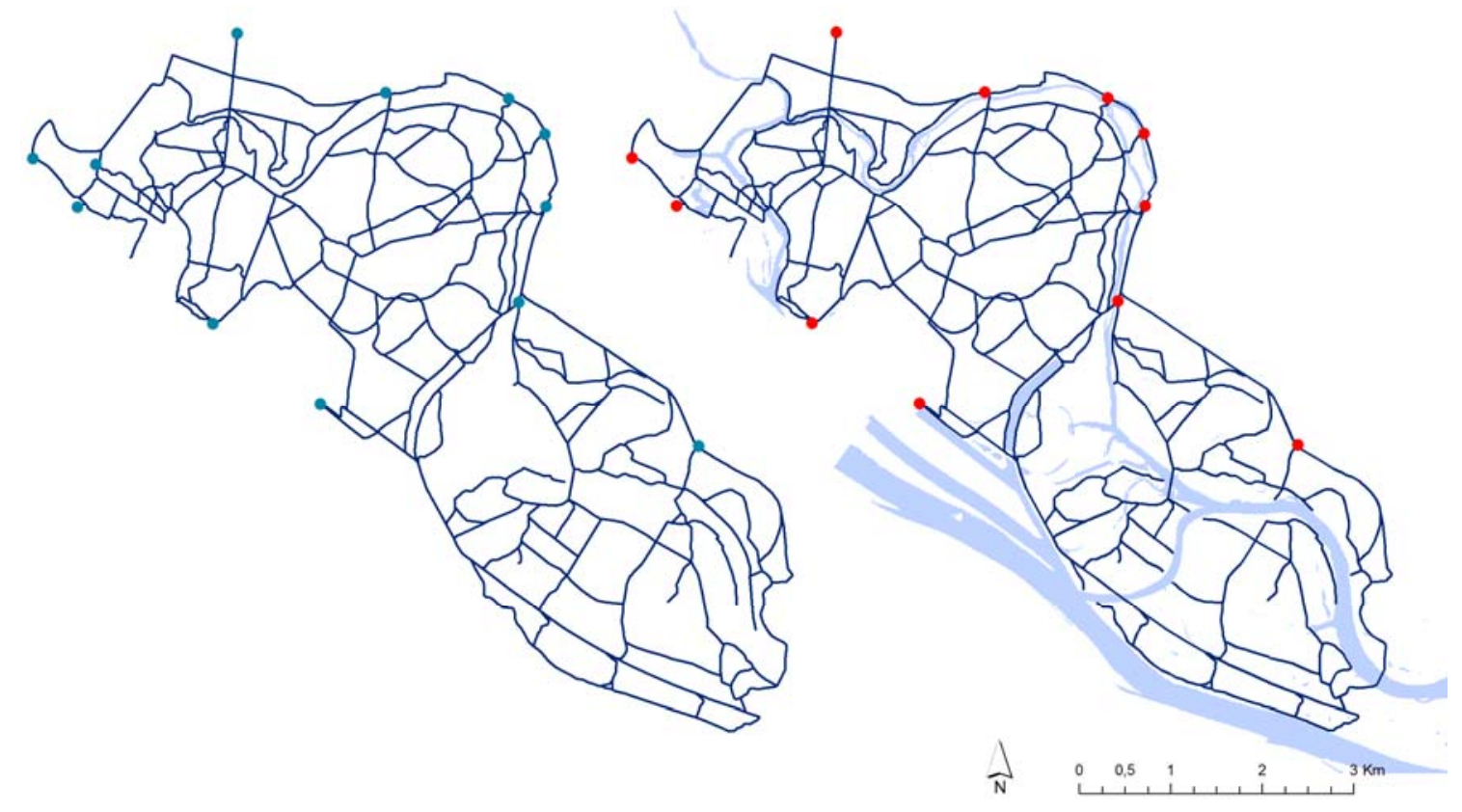

A)

Abb. 28: Inputdaten für die Simulation der Besucherströme in der Lobau. A) Wegenetz: Variante "Lobau A + Vorland A“ und 11 Eingänge; B) Wegenetz: Variante „Lobau B + Vorland A“ und 11 Eingänge 


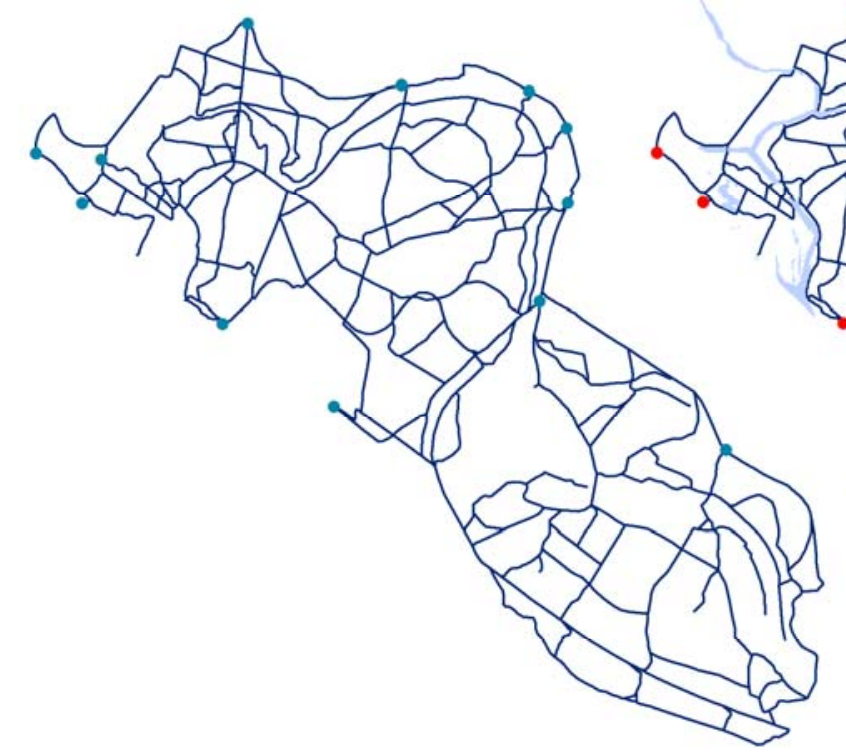

A)

Abb. 29: Inputdaten für die Simulation der Besucherströme in der Lobau. A) Wegenetz: Variante "Lobau A + Vorland A“ und 11 Eingänge; B) Wegenetz: Variante „Lobau B + Vorland A“ und 11 Eingänge

Im folgenden Schritt wurde eine Liste der relevanten Attribute und deren Ausprägungen für die Wegabschnitte festgelegt (Tab. 16). Dabei wurde darauf geachtet, dass die thematischen Attribute möglichst mit den abgefragten Attributen des Wahlmodels übereinstimmen (z.B. Wegbreite, s. Kapitel 4.1). Damit konnten die Präferenzen der Befragten hinsichtlich Landschaft und Erholungsinfrastruktur bestmöglich mit den realen bzw. hypothetischen Gegebenheiten, abgebildet in der digitalen, detaillierten Lobaukarte, im MASOOR-Model verknüpft werden. Im ArcGIS wurden jedem Wegabschnitt die entsprechenden Attribute zugewiesen. Anschließend wurden alle räumlichen und thematischen Daten kartographisch dargestellt. Abb. 30 bis Abb. 36 veranschaulichen die Eigenschaften des Wegenetzes für die Simulationsvarianten „Lobau A“ und „Lobau B“. Weitere Abbildungen (Abb. 37 bis Abb. 43) legen das Hauptaugenmerk auf die Ausgestaltung des LobauVorlands. 


\begin{tabular}{|c|c|}
\hline Attribute der Wegabschnitte & Attributkategorien / Werte \\
\hline \multicolumn{2}{|l|}{ A) Ebene Weg } \\
\hline Wegbreite & $\begin{array}{l}\text { 1) } 0,8 \mathrm{~m} \\
\text { 2) } 2 \mathrm{~m} \\
\text { 3) } 3 \mathrm{~m} \\
\text { 4) } 4 \mathrm{~m}\end{array}$ \\
\hline Wegbelag & $\begin{array}{l}\text { 1) Asphalt } \\
\text { 2) Schotter } \\
\text { 3) Erde }\end{array}$ \\
\hline Wegpflanzung & $\begin{array}{l}\text { 1) keine Wegbegleitbepflanzung } \\
\text { 2) Baumreihe } \\
\text { 3) Allee }\end{array}$ \\
\hline \multicolumn{2}{|l|}{ B) Ebene Landschaft } \\
\hline Landschaftstypus & $\begin{array}{l}\text { 1) Wald } \\
\text { 2) Wiese } \\
\text { 3) Heißlände } \\
\text { 4) Acker } \\
\text { 5) Acker \& Wiese } \\
\text { 6) Wald \& Heißlände } \\
\text { 7) Wiese \& Wald } \\
\text { 8) Sukzessionsfläche \& Wald } \\
\text { 9) Wald \& Wasser } \\
\text { 10) Wasser } \\
\text { 11) Brachen \& Wald } \\
\text { 12) Siedlungen } \\
\text { 13) Siedlungen \& Wald } \\
\text { 14) Brachen }\end{array}$ \\
\hline Hintergrund - Horizont & $\begin{array}{l}\text { 0) Waldkulisse in der Nähe }(<150 \mathrm{~m}) \\
\text { 3) Waldkulisse in größerer Entfernung (ca. } 300 \mathrm{~m}) \\
\text { 4) Siedlung mit Strommast }\end{array}$ \\
\hline \multicolumn{2}{|c|}{ C) Ebene Attraktionen und Lenkung } \\
\hline Gewässer - Attraktionen & $\begin{array}{l}\text { 0) Kein Ausblick auf Gewässer } \\
\text { 1) Ausblick auf Gewässer }\end{array}$ \\
\hline Infrastruktur-Attraktion & $\begin{array}{l}\text { 1) Keine zusätzliche Infrastruktur } \\
\text { 3) Wildbadeplatz mit Liegewiese } \\
\text { 4) Gasthaus mit Gastgarten }\end{array}$ \\
\hline \multicolumn{2}{|l|}{ D) Sonstiges } \\
\hline Path_ID * & ID-Nummer (Schlüsselfeld) \\
\hline Länge & Wert (in Meter) \\
\hline
\end{tabular}

Tab. 16: Liste der thematischen Attribute des Wegenetzes. 


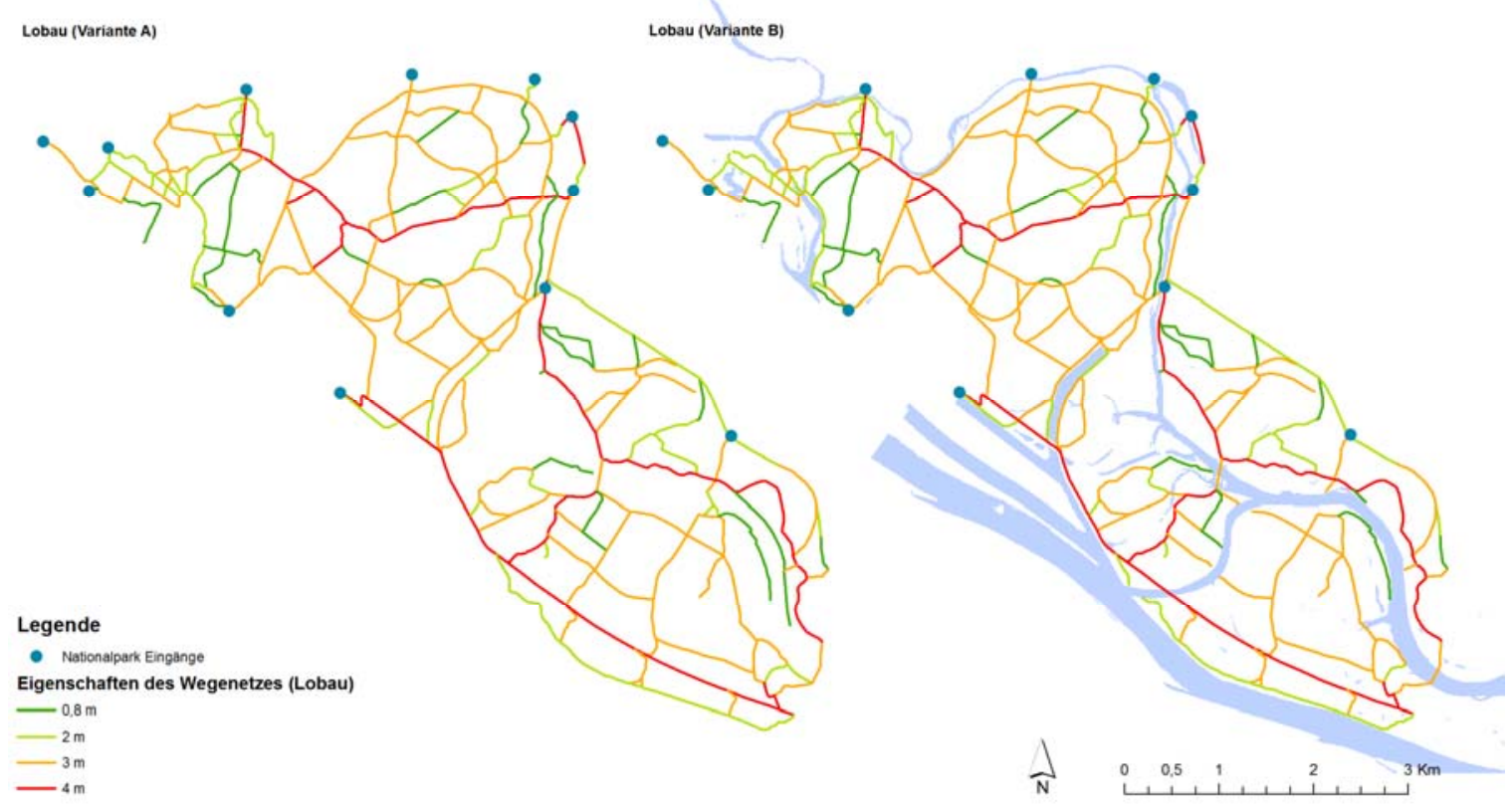

Abb. 30: Thematische Eigenschaften des Lobau-Wegenetzes: Wegbreite

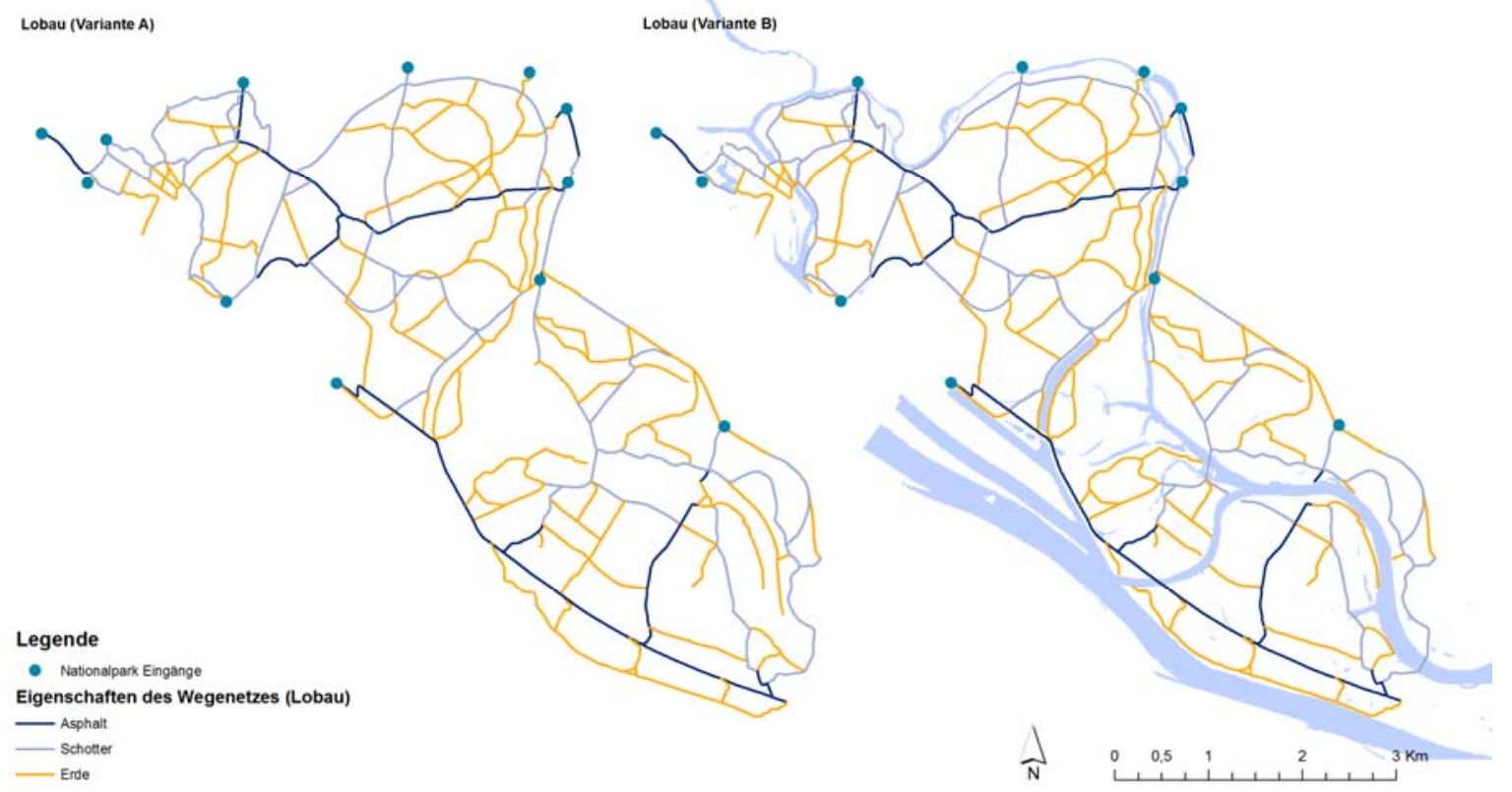

Abb. 31: Thematische Eigenschaften des Lobau-Wegenetzes: Wegbelag 


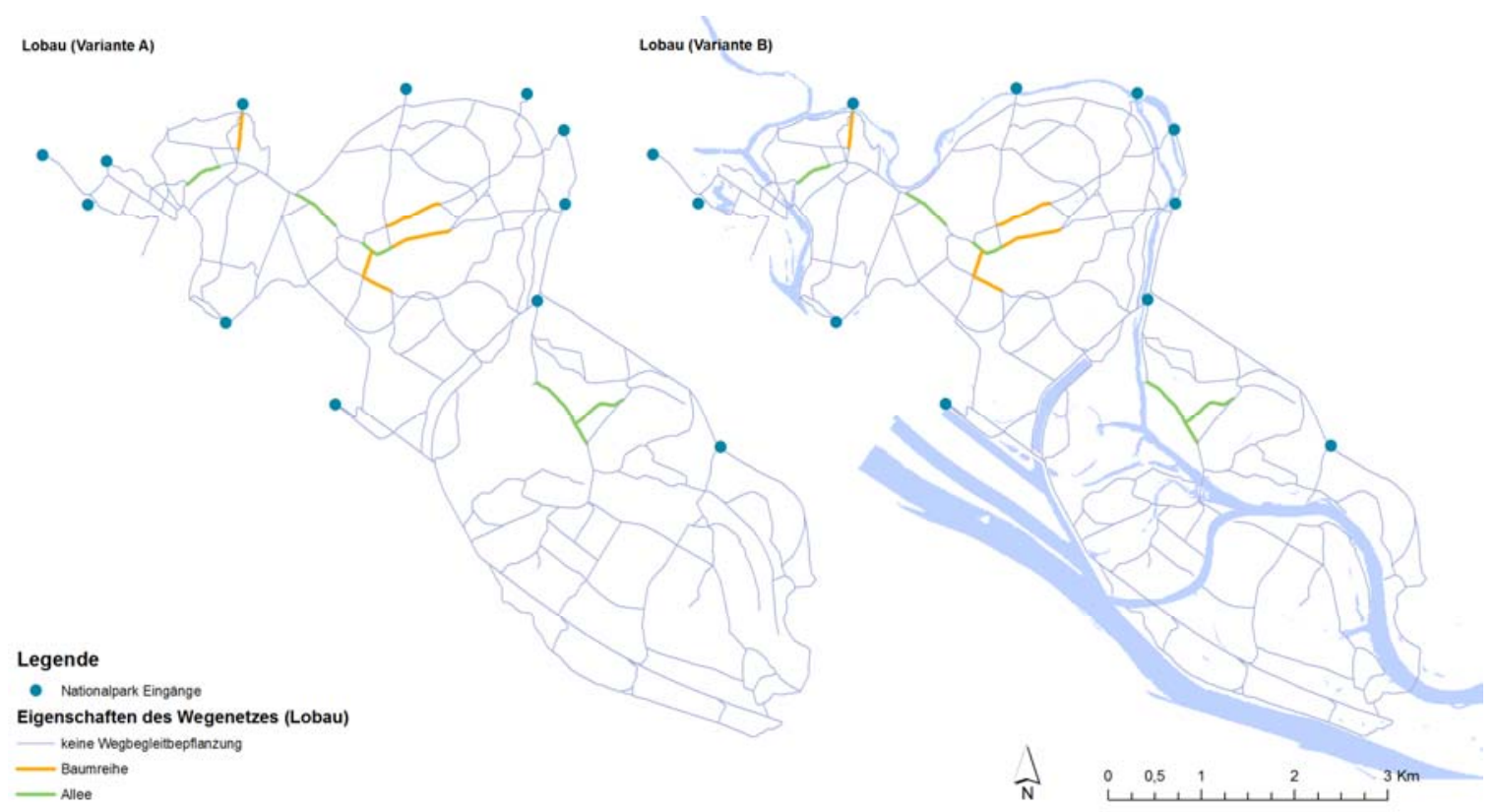

Abb. 32: Thematische Eigenschaften des Lobau-Wegenetzes: Wegbegleitbepflanzung

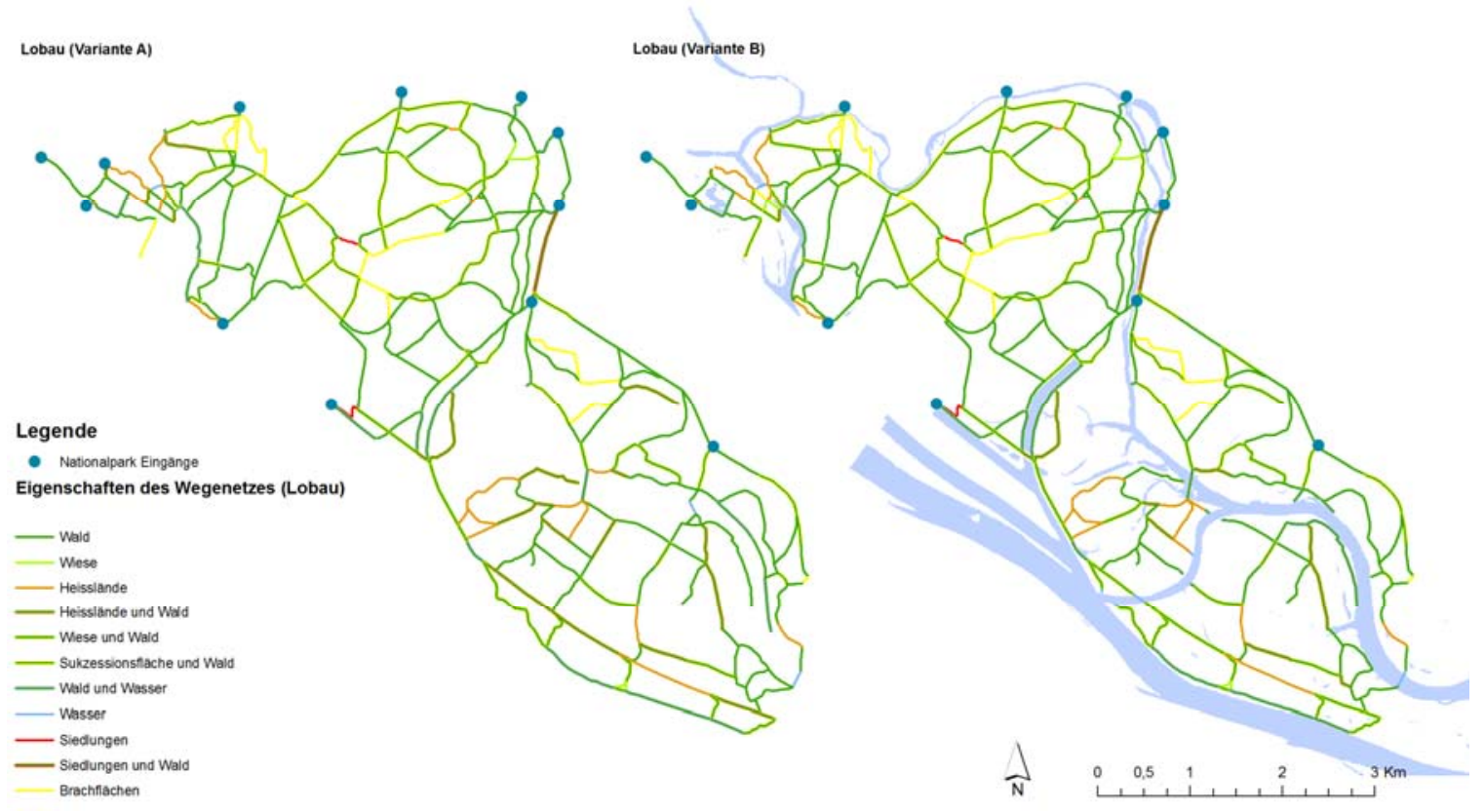

Abb. 33: Thematische Eigenschaften des Lobau-Wegenetzes: Landschaftstypus 


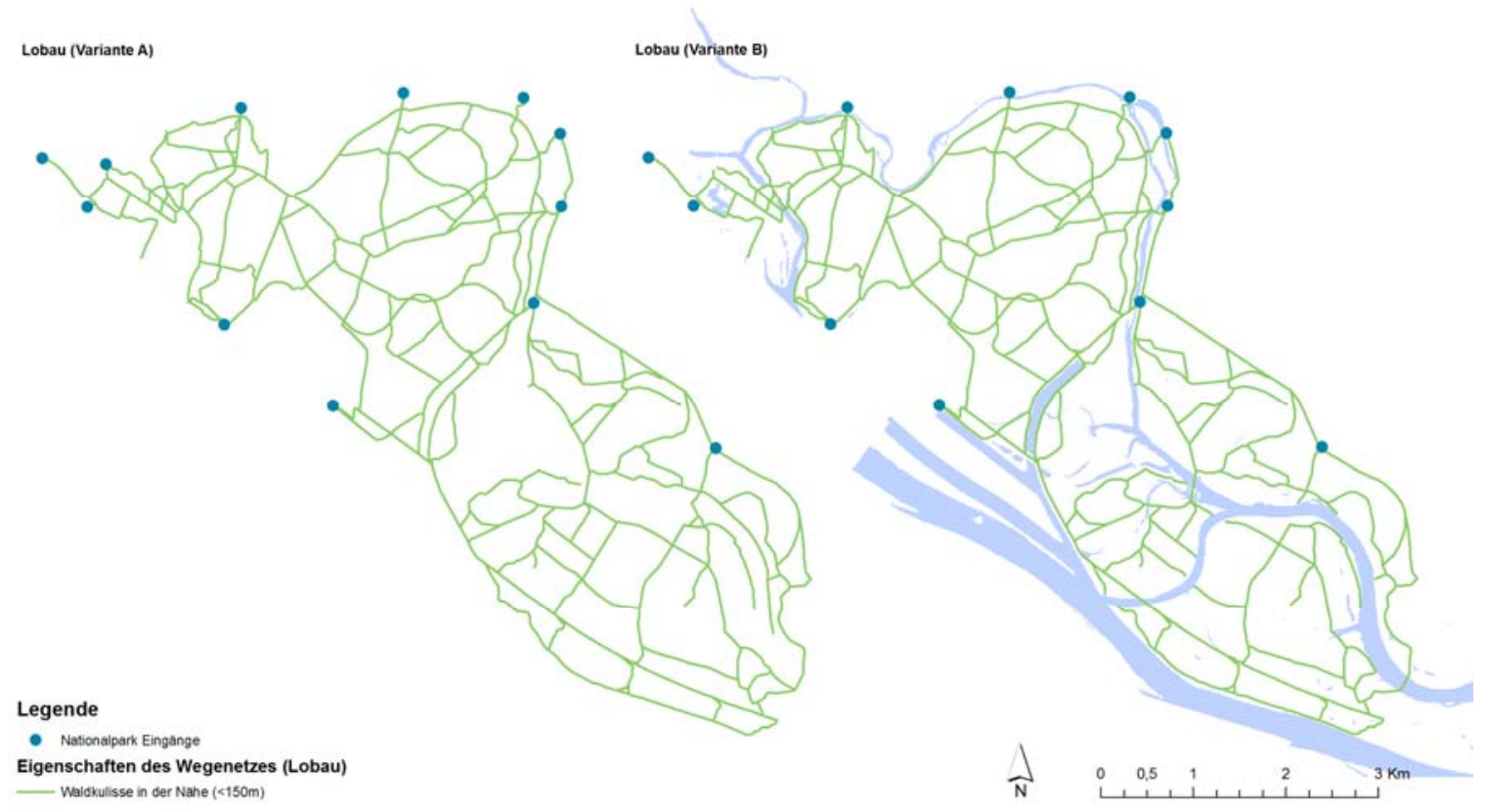

Abb. 34: Thematische Eigenschaften des Lobau-Wegenetzes: Hintergrund / Horizont

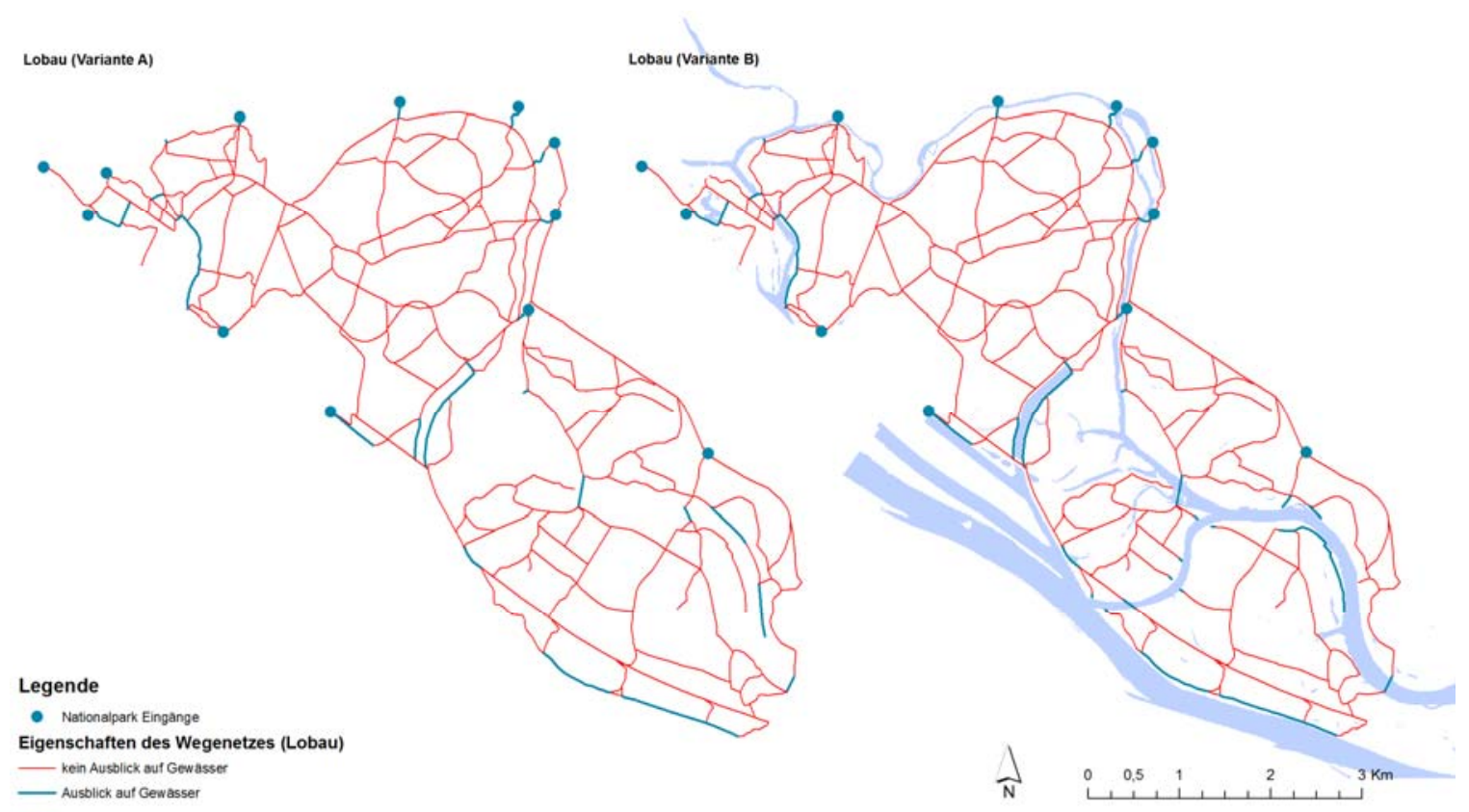

Abb. 35: Thematische Eigenschaften des Lobau-Wegenetzes: Ausblick auf Gewässer 


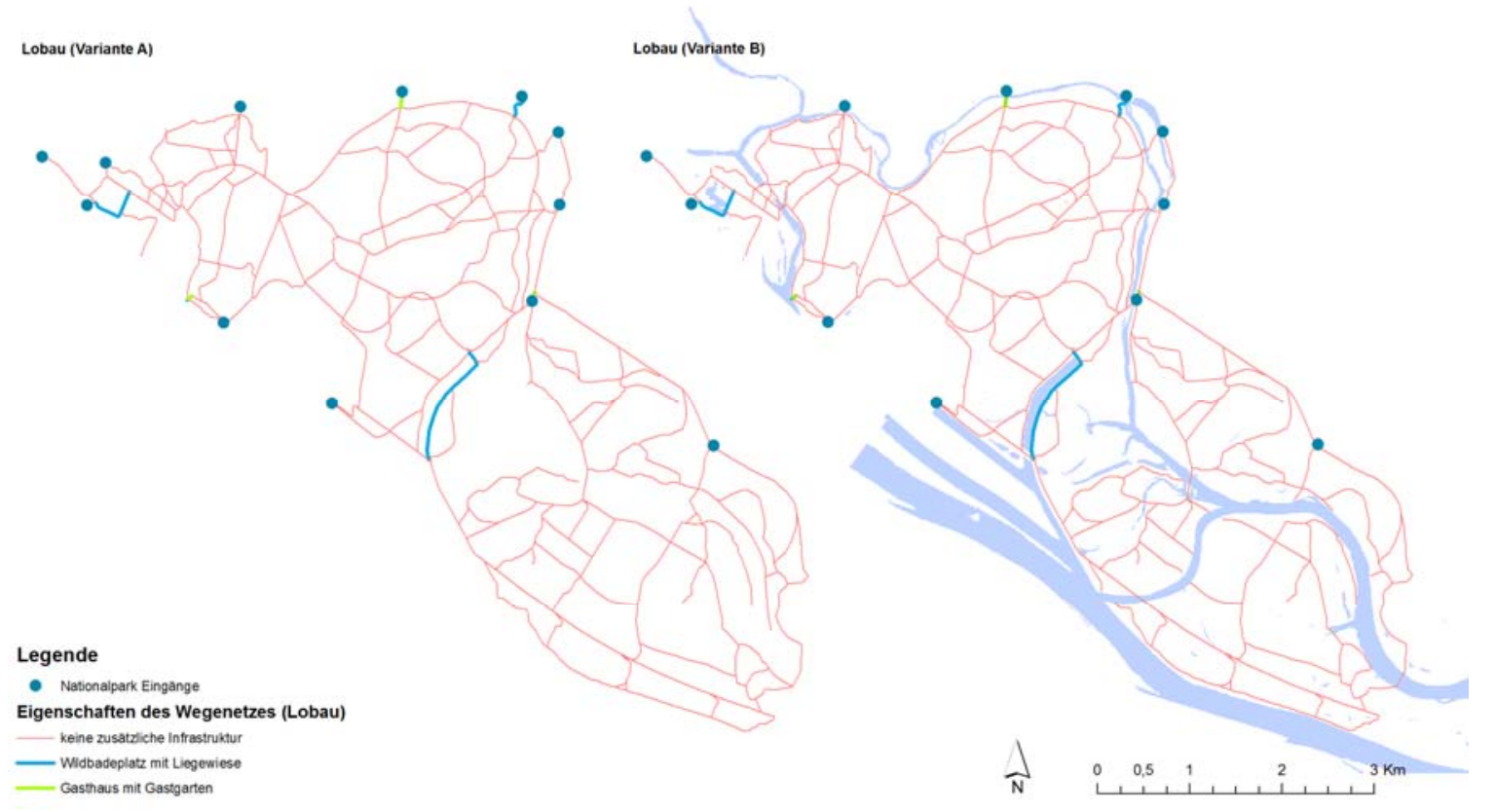

Abb. 36: Thematische Eigenschaften des Lobau-Wegenetzes: Infrastruktur

Die Ausgestaltung des Lobau-Vorlandes wurde in ArcGIS folgenderweise formalisiert: der Verlauf der geplanten Rad- und Wanderwege wurde digitalisiert; in Variante A (lineare Variante) wurden vor allem die bestehenden Landschaftskategorien wie Acker-Wiese berücksichtigt. In Variante B (flächige Variante) wurde eine abwechslungsreiche Landschaft, die aus Wiesen und Wälder besteht definiert. Abb. 37 bis 43 zeigen das Wegenetz und dessen Eigenschaften für beide Entwicklungsszenarien. 
Lobau Vorland (Variante A)

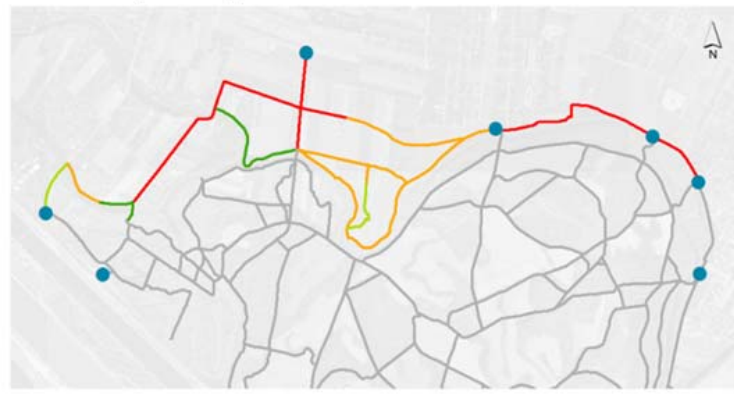

Lobau Vorland (Variante B)

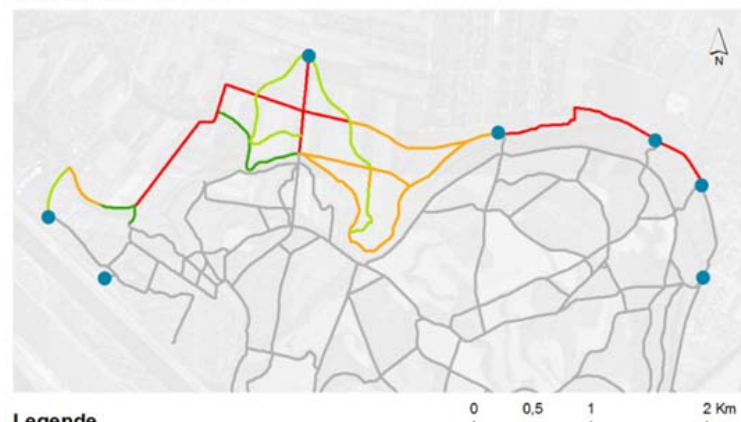

Legende

- Nationalpark Eingange

- Wegenetz innerhalb der Lobau

Eigenschaften des Wegenetzes im Lobau-Vorland

$-0.8 \mathrm{~m}$

$-2 \mathrm{~m}$

$3 \mathrm{~m}$

Abb. 37: Thematische Eigenschaften des Wegenetzes im Bereich des Lobau-Vorlands: Wegbreite

Lobau Vorland (Variante A

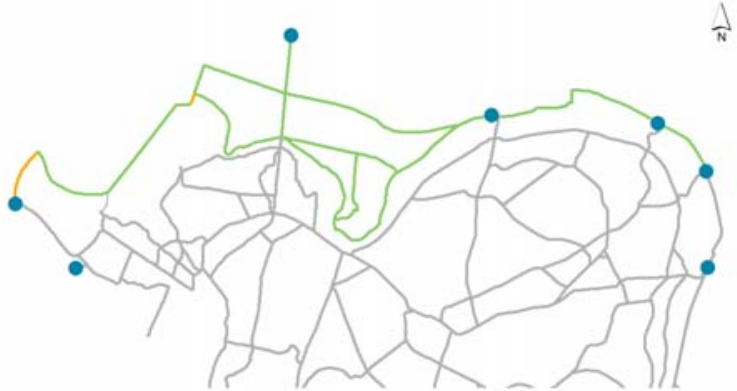

Lobau Vorland (Variante B)

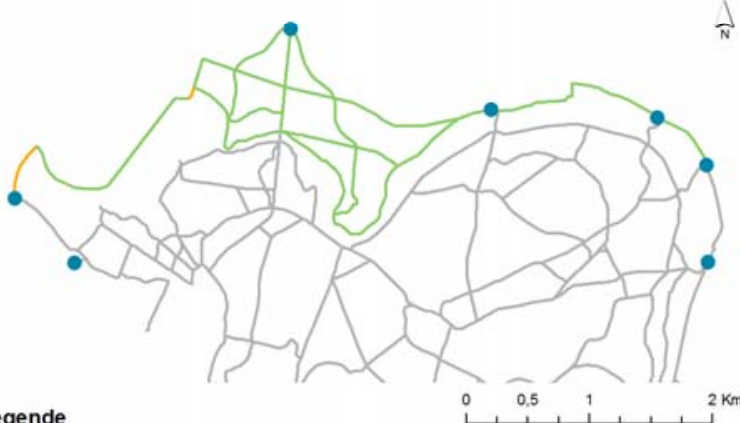

Legende $2 \mathrm{Km}$

- Nationalpark Eingange

- Wegenetz innerhalb der Lobau

Eigenschaften des Wegenetzes im Lobau-Vorland

- Baumreihe

Abb. 39: Thematische Eigenschaften des Wegenetzes im Bereich des Lobau-Vorlands: Wegbegleitbepflanzung
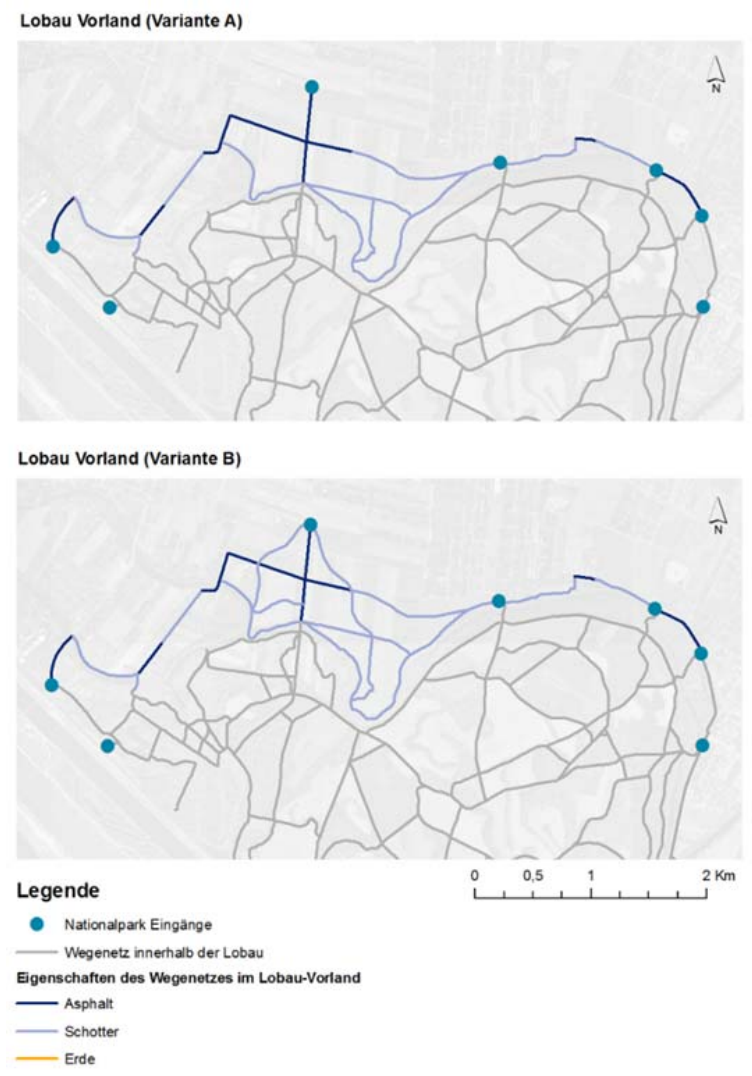

Abb. 38: Thematische Eigenschaften des Wegenetzes im Bereich des Lobau-Vorlands: Wegbelag

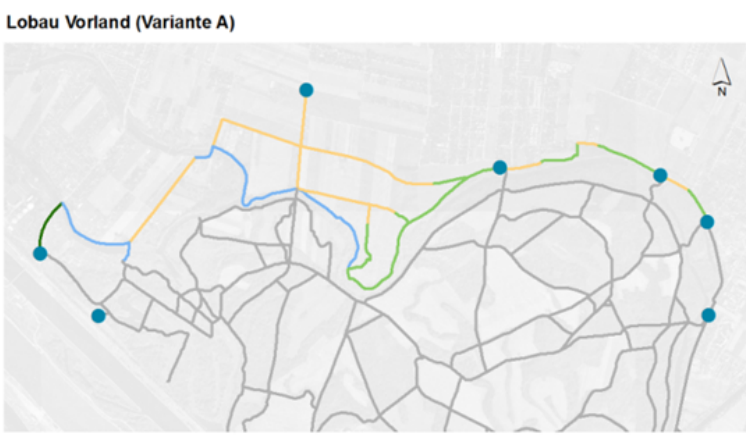

Lobau Vorland (Variante B)

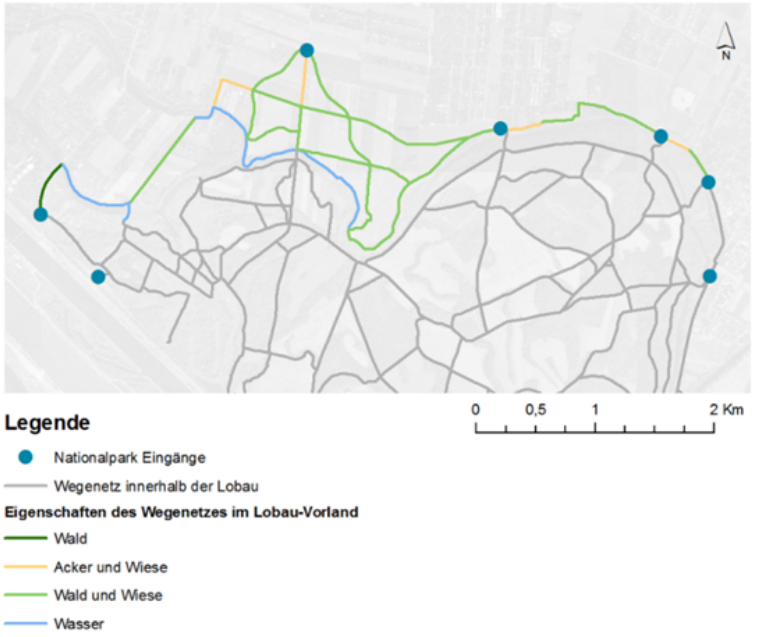

Abb. 40: Thematische Eigenschaften des Wegenetzes im Bereich des Lobau-Vorlands: Landschaftstypus 
Lobau Vorland (Variante A)

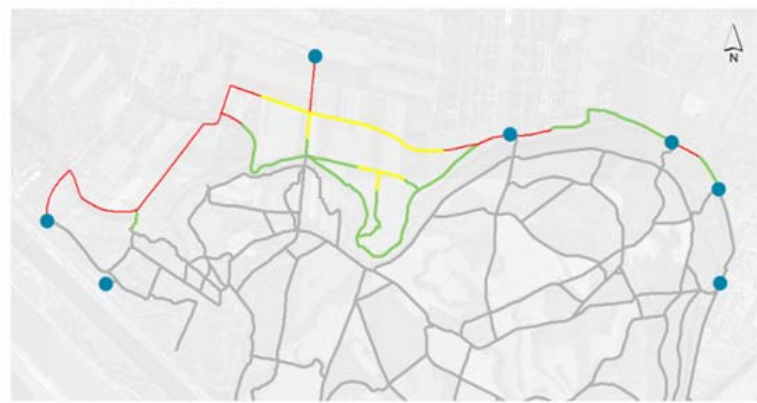

Lobau Vorland (Variante B)

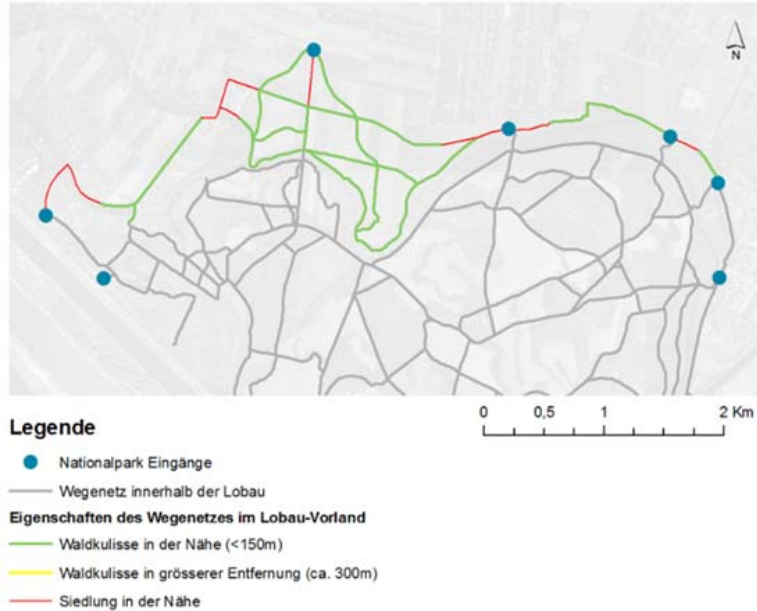

Abb. 41: Thematische Eigenschaften des Wegenetzes im Bereich des Lobau-Vorlands: Hintergrund / Horizont

Lobau Vorland (Variante A)

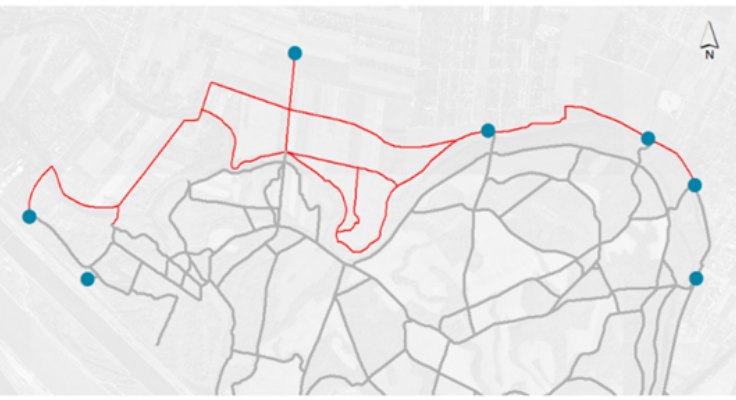

Lobau Vorland (Variante B)

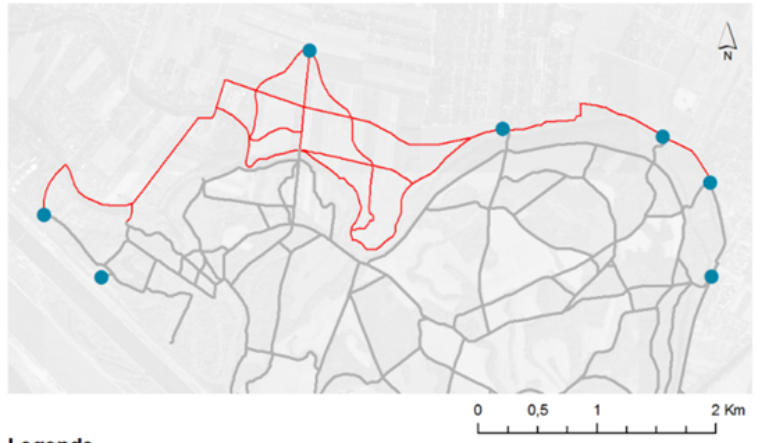

Legende

- Nationalpark Eingănge

Wegenetz innerhalb der Lobau

Eigenschaften des Wegenetzes im Lobau-Vorlan

- keine zusatzliche Infrastruktur

Abb. 43: Thematische Eigenschaften des Wegenetzes im Bereich des Lobau-Vorlands: Infrastruktur
Lobau Vorland (Variante A)

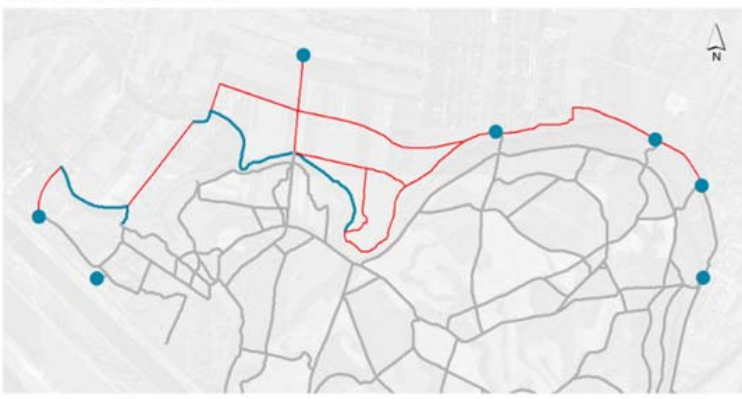

Lobau Vorland (Variante B)

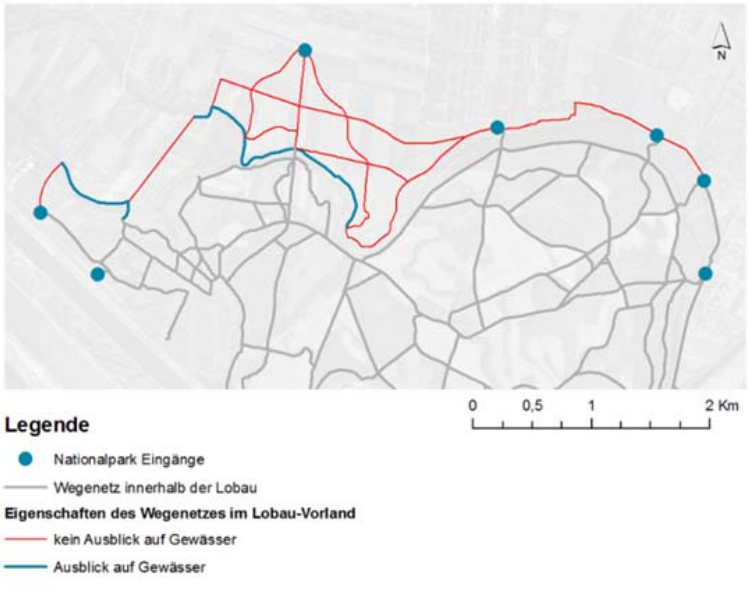

Abb. 42: Thematische Eigenschaften des Wegenetzes im Bereich des Lobau-Vorlands: Ausblick auf Gewässer 


\subsubsection{Definition der Agenten}

Folgende Arbeitsschritte waren für die Definition der Agentenpopulation erforderlich:

- Festlegung der Agententypen

- Bestimmung der prozentuellen Verteilung der Agententypen

- Definition der Bewegungsparameter / Präferenzen einzelner Agententypen

- Definition der Aufenthaltsdauer und der Geschwindigkeiten der einzelnen Agententypen (normale Verteilung der genannten Parameter)

- Bestimmung der Anzahl der hineingehenden Agenten (pro Eingang).

Festlegung der Agententypen und ihre prozentuelle Verteilung:

Die Resultate des Wahlmodels (s. Kap. 4.1) dienten als Grundlage für die Definition der Agententypen. Für die Simulationszwecke wurden die LobaubesucherInnen in sechs Gruppen eingeteilt: zu Fußgehende, Hundebesitzende, Nordic Walkende, Joggende, und zwei Typen von Radfahrenden, basierend auf den Ergebnissen der latent-class-Analyse. Es wurde angenommen, dass die Verteilung der Besuchertypen (Agententypen) gleich für alle Eingänge ist. Die beobachteten Unterschiede in der Besucherverteilung an einem Spitzentag und an einem Werktag wurden berücksichtigt. Die in Tab. 17 angeführten Anteile wurden als Eingabeparameter für die Simulation des Szenarios „0“ sowie für alle Szenarien für das Jahr 2025 verwendet.

\begin{tabular}{|l|l|r|r|}
\hline \multicolumn{2}{|c|}{ Agententyp } & $\begin{array}{c}\text { Verteilung an einem } \\
\text { Spitzentag [\%] }\end{array}$ & $\begin{array}{c}\text { Verteilung an einem } \\
\text { Werktag [\%] }\end{array}$ \\
\hline Typ 1 & FußgängerInnen & 23 & 24 \\
\hline Typ 2 & HundebesitzerInnen & 13 & 17 \\
\hline Typ 3 & Nordic WalkerInnen & 5 & 5 \\
\hline Typ 4 & JoggerInnen & 6 & 6 \\
\hline Typ 5 & RadfahrerInnen (Typ A) & 27 & 26 \\
\hline Typ 6 & RadfahrerInnen (Typ B) & 26 & 22 \\
\hline & Gesamt & 100 & 100 \\
\hline
\end{tabular}

Tab. 17: Prozentuelle Verteilung der Agententypen an einem Spitzentag und an einem Werktag.

Definition der Bewegungsparameter einzelner Agententypen

Die Festlegung der Bewegungsparameter der Agenten erfolgte nach einem gemeinsamen Schema für alle Agententypen. Für alle Typen wurden gleiche Bewegungsphasen festgelegt. Jede Route fing direkt in der Phase „Browse“ an und ging schrittweise in die Phasen „Directed“ und „Exit" über (Abb. 44). Die Eingangsphase „Entry“ wurde im MASOOR Model nicht verwendet, da es keine klaren Destinationen (Anziehungspunkte) in der Lobau gibt, die den kürzesten Weg zu einem bestimmten Ziel erfordern würden. Bisherige Studien in der Lobau haben gezeigt, dass die meisten Routen ohne konkreten Destinationsort zurückgelegt werden (Schrom-Feiertag et al., 2009). Die durchgeführten Routenanalysen im Testgebiet weisen zusätzlich darauf hin, dass die meisten BesucherInnen ihre Route am selben Ort beginnen und beenden, z.B. bei einem Parkplatz (Taczanowska, 2009). Diese empirischen Erkenntnisse wurden für die Definition der Eingabeparameter für die Simulation verwendet. In den dargestellten Simulationsvorgängen wurden keine „Traverse“-Routen berücksichtigt.

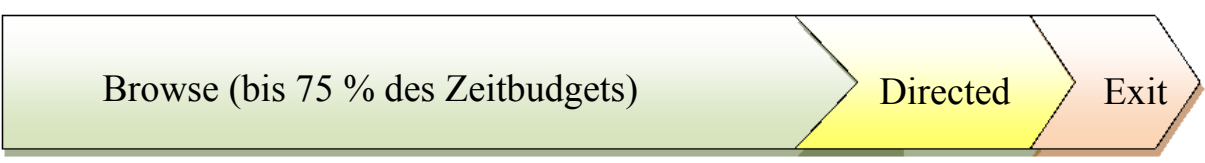

Abb. 44: Bewegungsphasen eines MASOOR-Agenten

Jede der drei definierten Bewegungsphasen unterscheidet sich hinsichtlich der Anwendung der Bewegungsalgorithmen (Tab. 18). Das Verhalten der Agenten in der „Browse“-Phase (75\% der Routendauer) hängt vor allem von den thematischen Eigenschaften des Wegenetzes ab, die mit den 
Wahlmodelergebnissen abgestimmt wurden. Darüber hinaus sind die Agenten in dieser Bewegungsphase auf die Anwesenheit anderer Erholungssuchenden empfindlich. Sie ändern ungern ihre Gehrichtung und bevorzugen noch nicht besuchte Wegabschnitte. In den zwei letzten Bewegungsphasen verlieren die Wegtyppräferenzen an Bedeutung. Dafür werden die kürzesten Wege in Richtung Ausgang bevorzugt.

\begin{tabular}{|l|c|c|c|c|}
\hline Agenten Bewegungsphase: & 1) ENTRY & 2) BROWSE & 3) DIRECTED & 4) EXIT \\
\hline MASOOR Algorithmus: & & & & \\
\hline U-turn & 0 & 1 & 0 & 0 \\
\hline Local heading & 0 & 1 & 0 & 0 \\
\hline Path type & 0 & 4 & 2 & 0 \\
\hline Path history & 0 & 1 & 0 & 0 \\
\hline Node history & 0 & 0 & 0 & 0 \\
\hline Global heading & 0 & 0 & 2 & 2 \\
\hline Shortest distance & 0 & 0 & 0 & 0 \\
\hline Path crowding & 0 & 2 & 1 & 0 \\
\hline In time & 0 & 0 & & \\
\hline
\end{tabular}

Tab. 18: Relative Wichtigkeit der MASOOR Bewegungsparameter für jede Agentenbewegungsphase (je höher ein Wert in der Tabelle, desto stärker ist der Einfluss eines Algorithmus; „0“ = Algorithmus wurde nicht verwendet).

Die Unterschiede zwischen den Agententypen liegen hauptsächlich in der Definition der Präferenzen für die einzelnen Wegetypen. Die Präferenzen basieren auf den Ergebnissen des Wahlmodells und wurden für jeden Wegtyp und Agententyp berechnet. Tab. 19 fasst die detaillierten Präferenzwerte für jeden Agententyp zusammen. In der MASOOR Plattform kann nur ein Attribut als Basis für die Wegtyppräferenzen ausgewählt werden. Um die Ergebnisse des Wahlmodels mit den heterogenen Umwelteigenschaften berücksichtigen zu können, war es notwendig, eine neue Variable zu erstellen, die alle erforderliche Parameter beinhaltet. Die unten dargelegte Gleichung (Abb. 45) erläutert die Zusammenstellung der Werte und erklärt die Bedeutung des neuen Wegtypattributs.

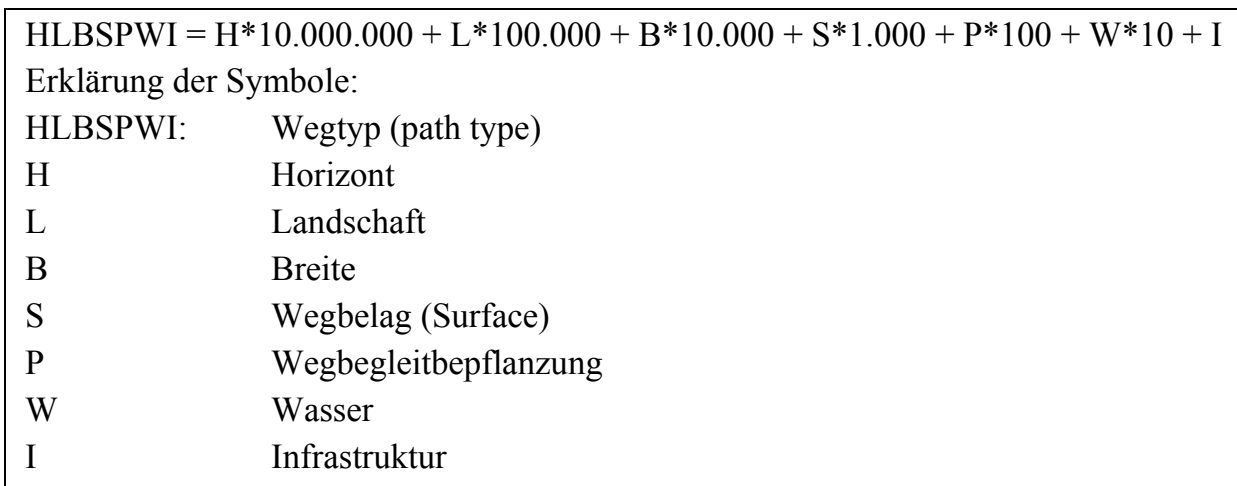

Beispiel: HLBSPWI Wert „113001“ definiert einen Wegabschnitt mit folgenden Eigenschaften: Horizont (0): Waldkulisse in der Nähe / Landschaft (1): Wald / Breite (1): 0,8 m / Belag (3): Erde / Wegbegleitbepflanzung (0): keine / Wasser (0): kein Ausblick auf Gewässer / Infrastruktur (1): keine zusätzliche Infrastruktur.

Abb. 45: Berechnung des neuen Wegtyp-Attributs. Zusammenstellung mehrerer Eigenschaften in eine kategorische Variable. 


\begin{tabular}{|l|c|c|c|c|c|c|}
\hline $\begin{array}{c}\text { Wegtyp } \\
\text { (HLBSPWI) }\end{array}$ & $\begin{array}{c}\text { VALUE_Ty } \\
\text { p1 }\end{array}$ & $\begin{array}{c}\text { VALUE_ } \\
\text { Typ2 }\end{array}$ & $\begin{array}{c}\text { VALUE_ } \\
\text { Typ3 }\end{array}$ & $\begin{array}{c}\text { VALUE_ } \\
\text { Typ4 }\end{array}$ & $\begin{array}{c}\text { VALUE_ } \\
\text { Typ5 }\end{array}$ & $\begin{array}{c}\text { VALUE_ } \\
\text { Typ6 }\end{array}$ \\
\hline 113001 & 0.46 & 0.15 & 0.14 & 0.38 & 0.00 & 0.00 \\
\hline 122001 & 0.24 & 0.15 & 0.14 & 0.15 & 2.28 & 0.49 \\
\hline 122004 & 0.45 & 0.15 & 0.14 & 0.21 & 2.28 & 0.56 \\
\hline 123001 & 0.46 & 0.15 & 0.14 & 0.15 & 2.28 & 0.41 \\
\hline 131001 & 0.00 & 0.15 & 0.14 & 0.63 & 1.58 & 1.06 \\
\hline 132001 & 0.24 & 0.15 & 0.14 & 0.63 & 1.58 & 1.11 \\
\hline 132013 & 1.28 & 0.15 & 0.92 & 1.18 & 3.70 & 1.63 \\
\hline 133001 & 0.46 & 0.15 & 0.14 & 0.63 & 1.58 & 1.03 \\
\hline
\end{tabular}

Tab. 19: MASOOR Inputparameter - Beispiele für Wegtyppräferenzen nach Agententypen

Die folgenden Karten (Abb. 46 bis Abb. 51) veranschaulichen einige Beispiele der Wegtyppräferenzen für verschiedene Agententypen. Die Präferenzen basieren auf den Ergebnissen des Wahlmodells.

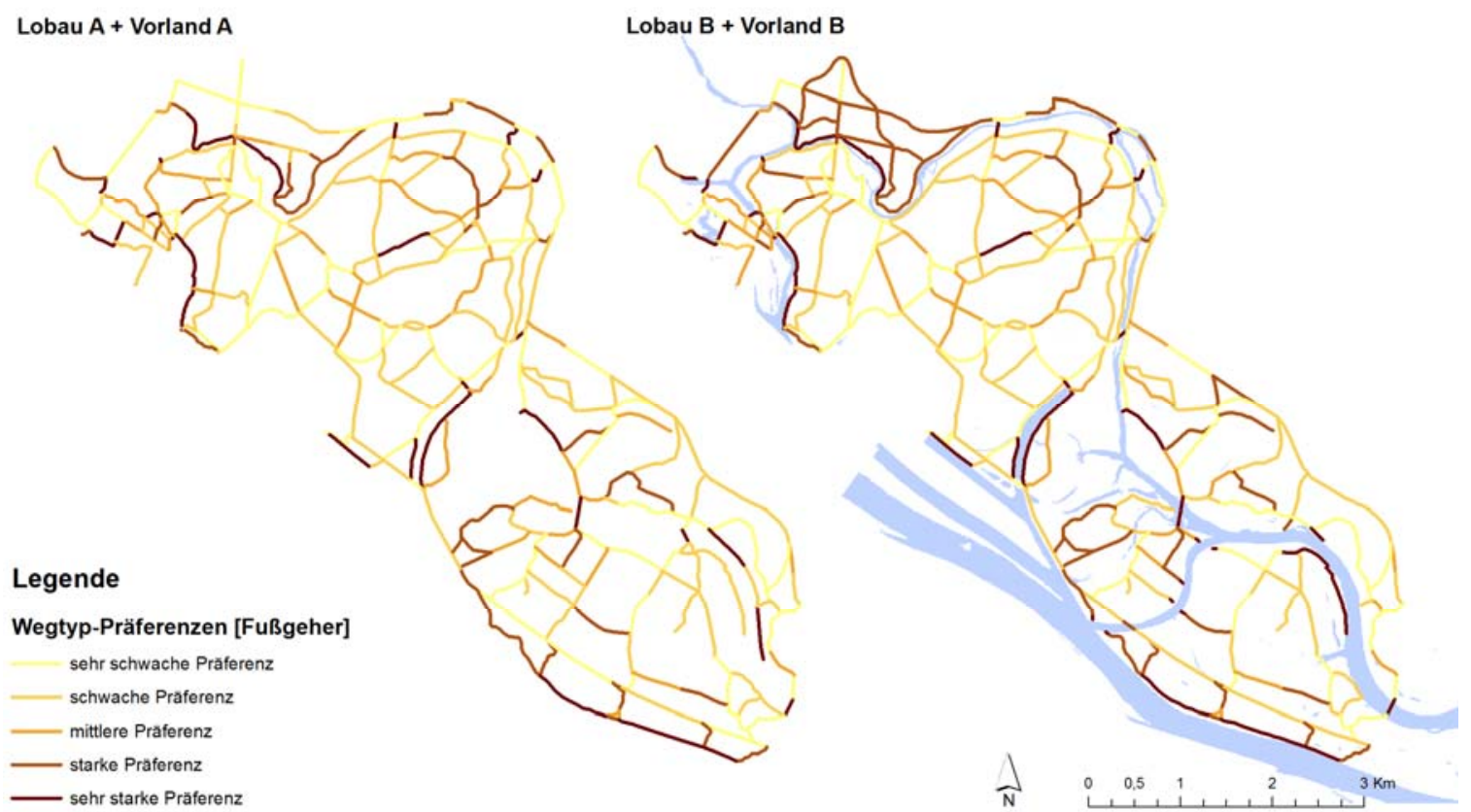

Abb. 46: Die relativen Wegtyppräferenzen der Agenten (Typ 01 - Fußgeher) für verschiedene Managementszenarien innerhalb der Lobau (Variante A und B) und im Bereich des Lobau-Vorlands (Variante A und B). Es ist ersichtlich, dass die FußgängerInnen die flächige Ausgestaltungsvariante des Vorlandes (Wald und Wiesen) bevorzugen. 


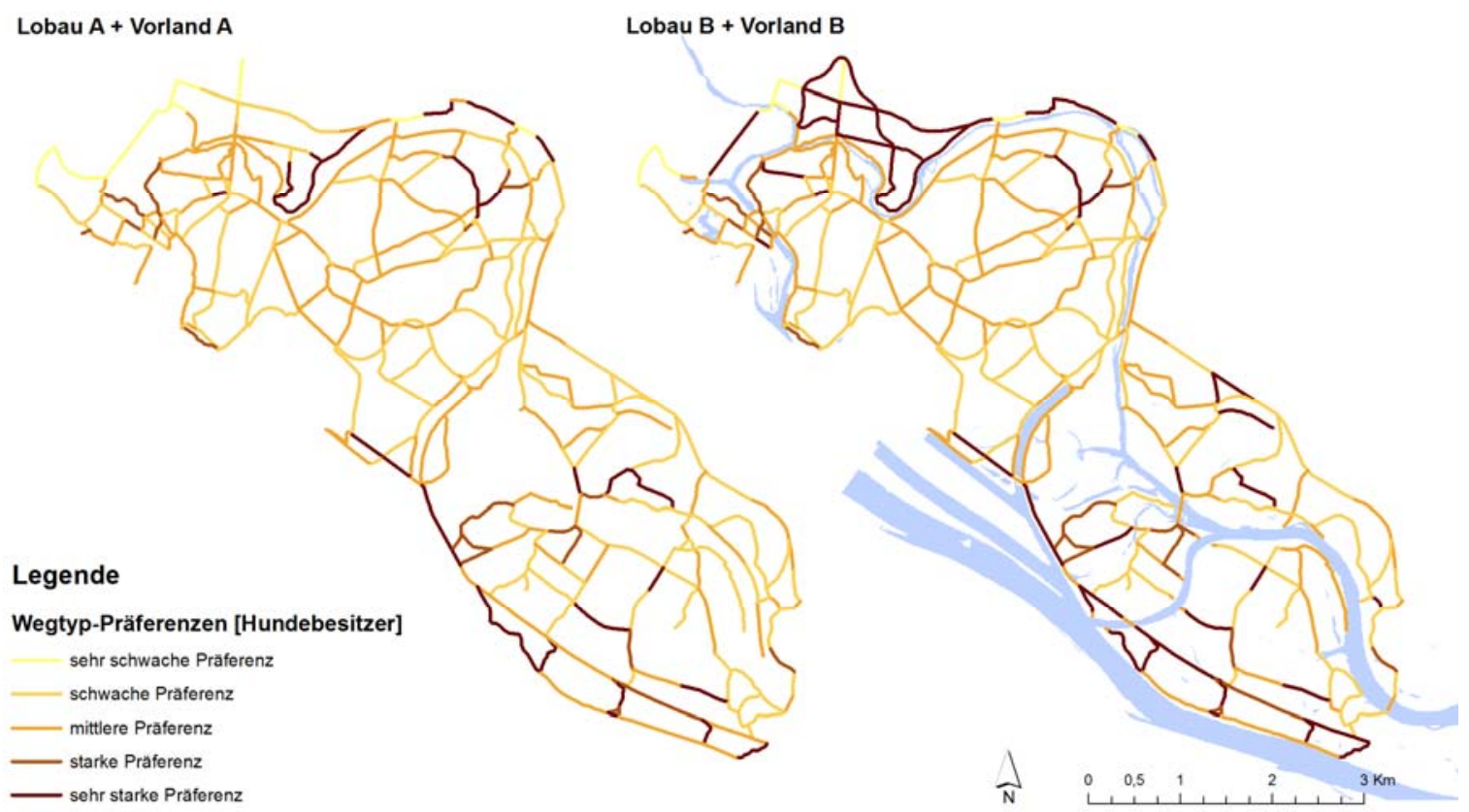

Abb. 47: Die relativen Wegtyppräferenzen der Agenten (Typ 02 - Hundebesitzer) für verschiedene Managementszenarien innerhalb der Lobau (Variante A und B) und im Bereich des Lobau-Vorlands (Variante A und B).

Lobau A + Vorland A

\section{Legende}

Wegtyp-Präferenzen [Nordic Walker] sehr schwache Praferenz

schwache Praferenz

mittlere Praferenz

- starke Präferenz

- sehr starke Praferenz
Lobau B + Vorland B

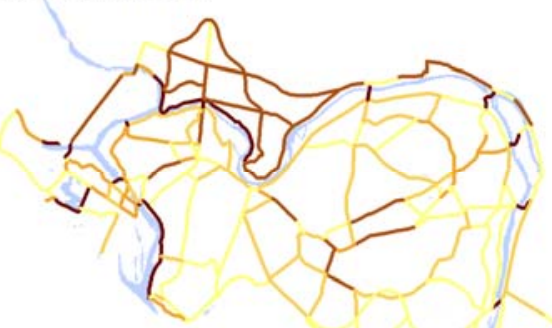

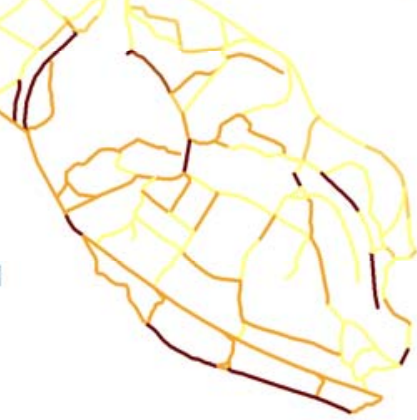

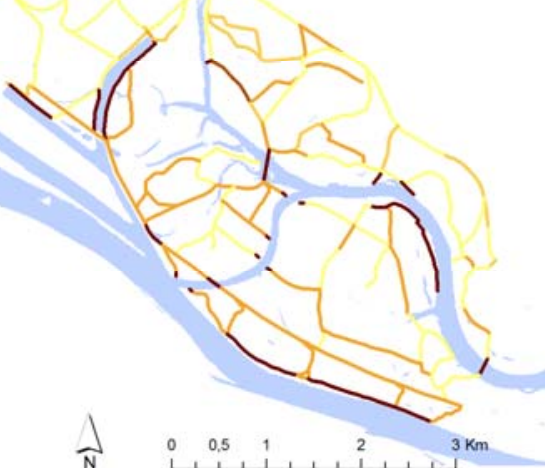

Abb. 48: Die relativen Wegtyppräferenzen der Agenten (Typ 03- Nordic WalkerInnen) für verschiedene Managementszenarien innerhalb der Lobau (Variante A und B) und im Bereich des Lobau-Vorlands (Variante A und B). 
Lobau A + Vorland A

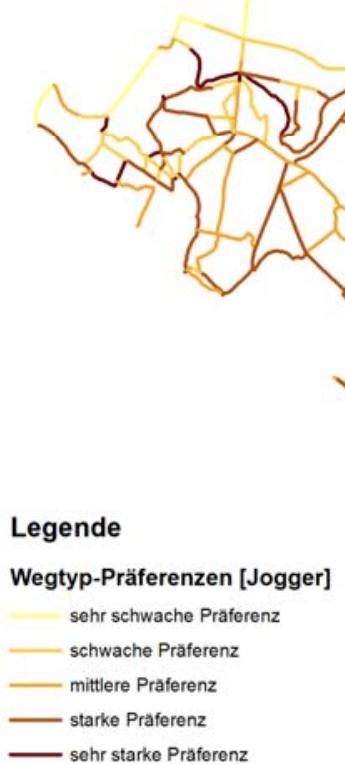

Lobau B + Vorland B

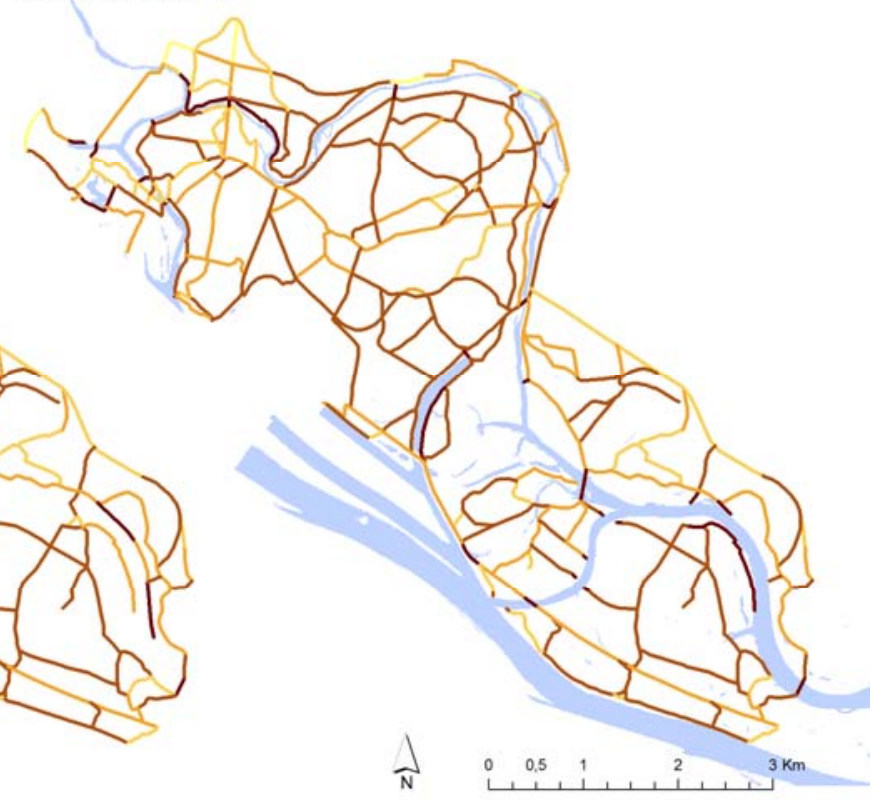

Abb. 49: Die relativen Wegtyppräferenzen der Agenten (Typ 04 - JoggerInnen) für verschiedene Managementszenarien innerhalb der Lobau (Variante A und B) und im Bereich des Lobau-Vorlands (Variante A und B).

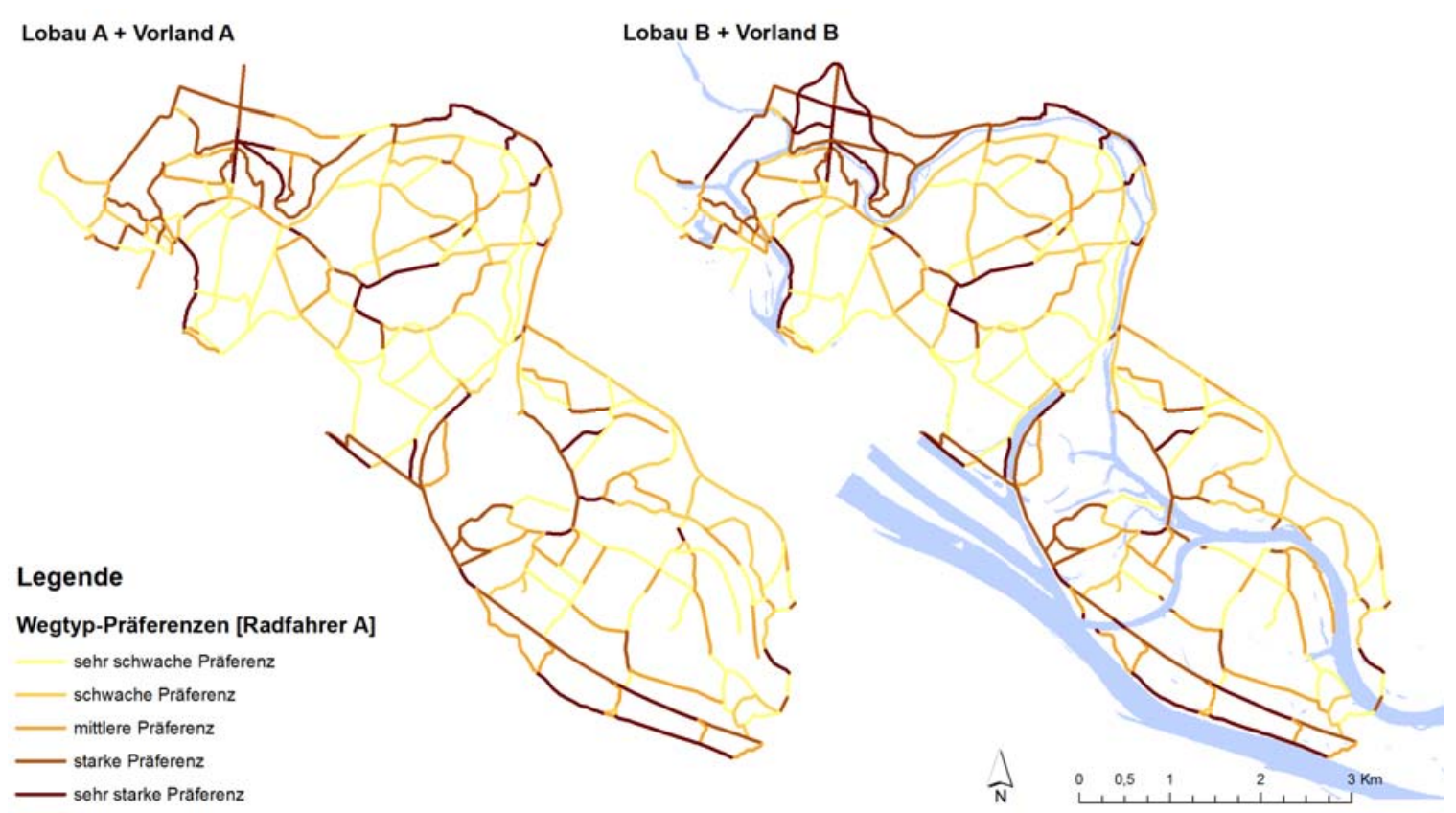

Abb. 50: Die relativen Wegtyppräferenzen der Agenten (Typ 05- Radfahrerlnnen A) für verschiedene Managementszenarien innerhalb der Lobau (Variante A und B) und im Bereich des Lobau-Vorlands (Variante A und B). 


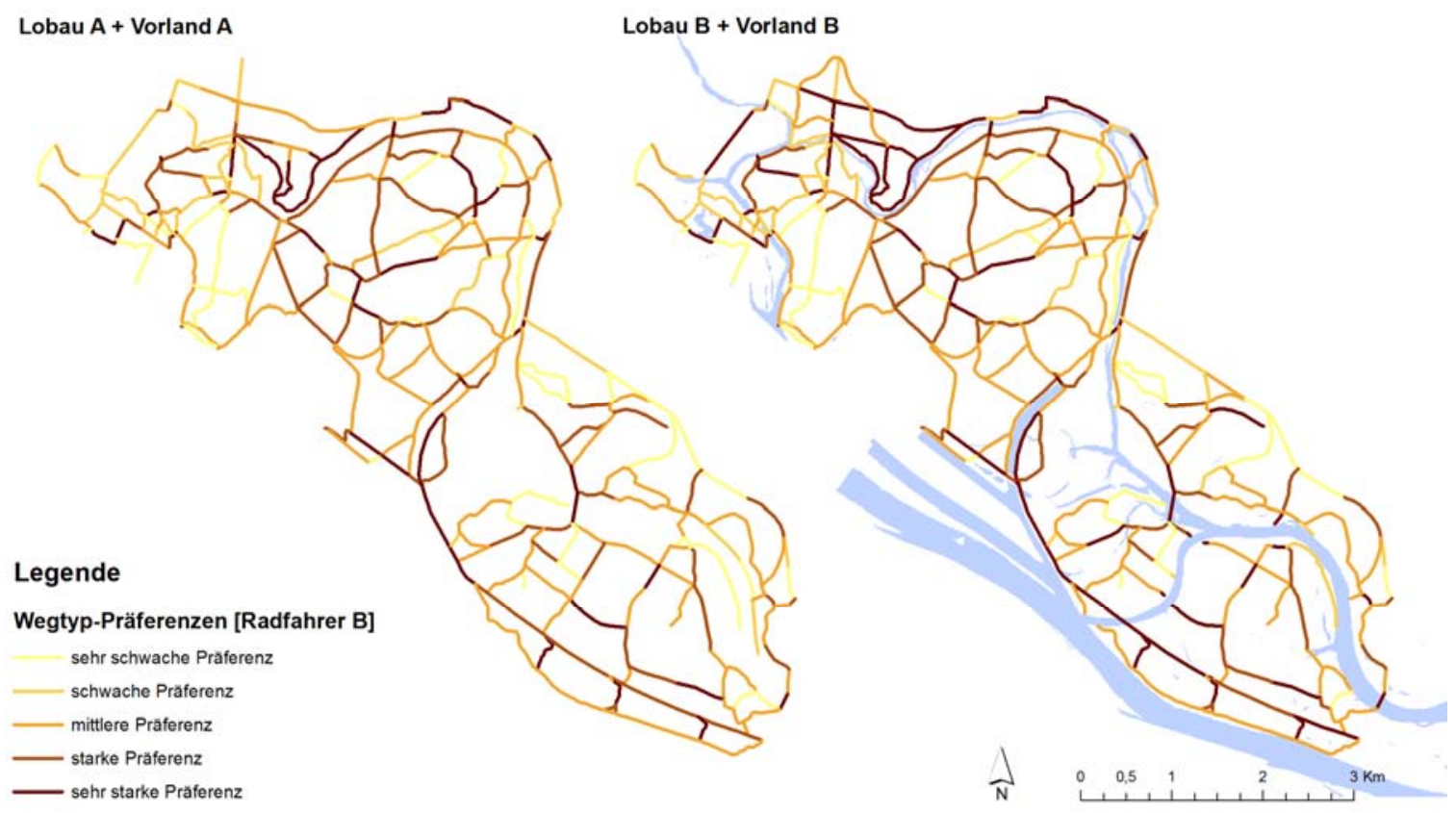

Abb. 51: Die relativen Wegtyppräferenzen der Agenten (Typ 06 - RadfahrerInnen B) für verschiedene Managementszenarien innerhalb der Lobau (Variante A und B) und im Bereich des Lobau-Vorlands (Variante A und B).

Definition der Aufenthaltsdauer und der Geschwindigkeiten der einzelnen Agententypen

Die Aufenthaltsdauer (Zeitbudget) und die Geschwindigkeit der Fortbewegung wurde für jeden Agententypen für die Simulation definiert (Tab. 20). Diese Daten wurden aus den Routenbefragungen in der Lobau gewonnen. RadfahrerInnen legten weitere Stecken in der Lobau zurück als beispielsweise FußgängerInnen.

\begin{tabular}{|l|l|r|r|r|r|r|r|}
\hline \multirow{2}{*}{} & \multicolumn{2}{c|}{ Routenlängen } & \multicolumn{2}{c|}{ Eingabeparameter für die Simulation } \\
\cline { 2 - 8 } & \multicolumn{2}{|c|}{ Länge (m) } & Geschwindigkeit (m/h) & \multicolumn{2}{c|}{ Dauer (Minuten) } \\
\hline \multicolumn{2}{|c|}{ Agententyp } & Mittelwert & St.Abw. & Mittelwert & \multicolumn{1}{c|}{ St.Abw. } & \multicolumn{1}{c|}{ Mittelwert } & St.Abw. \\
\hline Typ 01 & Fußgeher & 4653 & 2927 & 3500 & 500 & 80 & 59 \\
\hline Typ 02 & Hundebesitzer & 4264 & 2510 & 3500 & 500 & 73 & 50 \\
\hline Typ 03 & Nordic Walker & 6145 & 2352 & 4500 & 600 & 82 & 37 \\
\hline Typ 04 & Jogger & 6659 & 3679 & 7000 & 800 & 57 & 37 \\
\hline Typ 05 & Radfahrer (A) & 12185 & 7214 & 13000 & 1000 & 56 & 39 \\
\hline Typ 06 & Radfahrer (B) & 14566 & 9138 & 15000 & 1000 & 58 & 43 \\
\hline
\end{tabular}

Tab. 20: Wegelängen und Fortbewegungsgeschwindigkeit der LobaubesucherInnen als Eingabeparameter für die Simulation.

Anzahl der hineingehenden BesucherInnen (Agenten)

Um eine detaillierte Prognose der Besucherzahlen für einen Spitzentag im Jahr 2025 und für die 12 Lobaueingänge erstellen zu können, wurden folgende Daten verwendet:

- Beobachtetes Besucheraufkommen auf Basis der Besuchererhebungskampagne von 1999 (Arnberger et al., 2000; Brandenburg, 2001)

- Ergebnisse der Erreichbarkeitsanalyse (Wahl der Eingänge von jenen BesucherInnen, die in einer Entfernung von 2-4 km von der Lobau wohnen)

- Ergebnisse der Prognose über das Besucheraufkommen für das Jahr 2025 (Gesamtzahl)

Die Erfassung der Zuordnung der Eingangswahl und Besuchshäufigkeit in Abhängigkeit vom Wohnort war erforderlich, um vorhersagen zu können, welche Eingänge aufgrund der städtebaulichen Entwicklung künftig wie häufig frequentiert werden (Abb. 52). Dazu wurde insbesondere der Einzugsbereich innerhalb von 2 bis $4 \mathrm{~km}$ Entfernung von den Eingängen herangezogen, da hier ein Großteil der neuen EinwohnerInnen wohnen wird (z.B. Seestadt Aspern). 


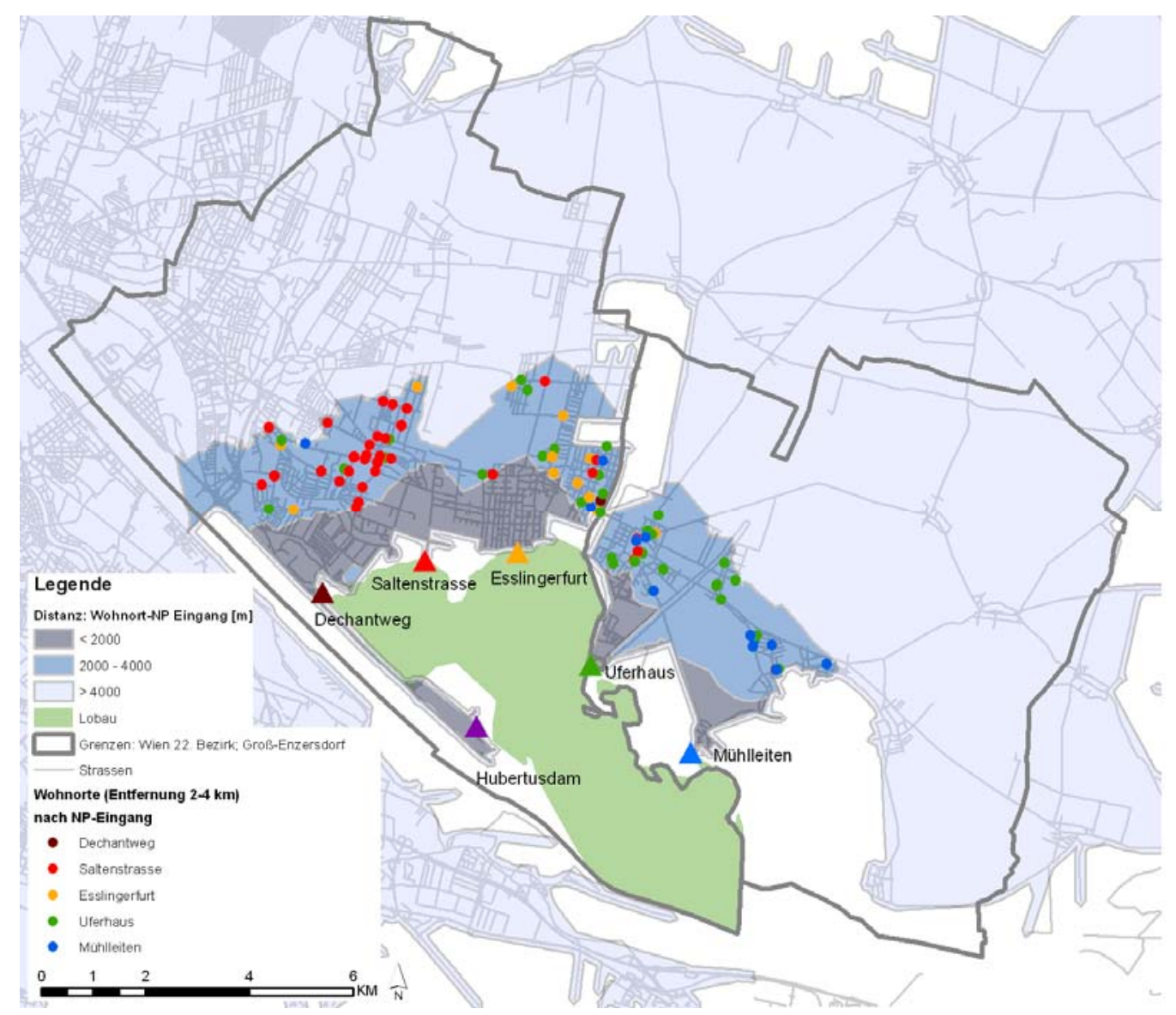

Abb. 52: Ergebnisse der Erreichbarkeitsanalyse für die Haupteingänge in die Lobau unter besonderer Berücksichtigung der Entfernungszone 2-4 km. (Datenbasis: Befragung „Perspektive LOBAU 2020“); Unterschiedliche Farben der Punktdaten ermöglichen eine Zuordnung der Wohnorte und der, von den BesucherInnen, benutzten Eingänge.

Die Abb. 53 stellt die Anteile der benutzten Eingänge jeweils für die BesucherInnen aus Wien und aus der Gemeinde Gross-Enzersdorf dar, die in einer Entfernung von 2 bis 4 km von einem Lobaueingang wohnen. $51 \%$ der Befragten aus Wien nutzten den Eingang Saltenstraße, während BesucherInnnen aus Groß-Enzersdorf v.a. den Eingang Uferhaus benützten, um in die Lobau zu gelangen.
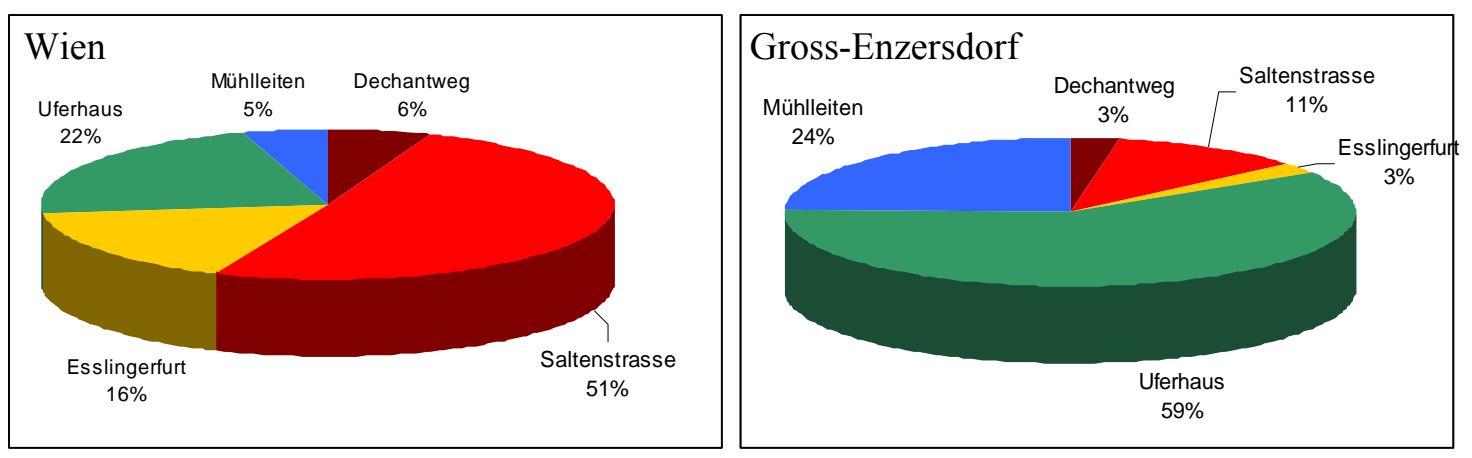

Abb. 53: Ergebnisse der Erreichbarkeitsanalyse für die Lobau-Haupteingänge unter besonderer Berücksichtigung der Entfernungszone 2-4 km. Wahl der Eingänge für die BesucherInnen aus Wien sowie aus der Gemeinde Gross-Enzersdorf. 
Aufgrund der Prognose der Besuchermenge für 2025 wurde diese auf die Untersuchungstage umgerechnet (Tab. 21).

\begin{tabular}{|c|l|r|r|}
\hline Jahr & \multicolumn{1}{|c|}{ Datenherkunft / Zeitabschnitt } & $\begin{array}{c}\text { Anzahl der } \\
\text { hineingehenden } \\
\text { BesucherInnen }\end{array}$ & \multicolumn{1}{c|}{$\begin{array}{c}\text { Anteil der } \\
\text { BesucherInnen }\end{array}$} \\
\hline 1999 & Erhebungskampagne [pro Jahr] & 600.000 & $100 \%$ \\
\hline 1999 & Spitzentag (März; Sonntag) & 6.424 & $1,1 \%$ \\
\hline 1999 & Werktag (Mittelwert 18.03; 29.07; Donnerstag) & 1.719 & $0,3 \%$ \\
\hline 2025 & Prognose: neue Besuche (Wien) [pro Jahr] & 471.000 & $79 \%$ \\
\hline 2025 & Prognose: neue Besuche (G-Enz) [pro Jahr] & 316.000 & $53 \%$ \\
\hline 2025 & Prognose [pro Jahr] & 1.387 .000 & $231 \%$ \\
\hline 2025 & Prognose [Spitzentag] & 14.850 & $1,1 \%$ \\
\hline 2025 & Prognose [Werktag] & 3.974 & $0,3 \%$ \\
\hline
\end{tabular}

Tab. 21: Besucheraufkommen im Jahr 1999 (Arnberger et al., 2000) und Prognose des Gesamtbesucheraufkommens je Besuchstag für 2025.

In einem zweiten Schritt wurde der Besuchermengenzuwachs auf die einzelnen Lobaueingänge verteilt (Tab. 22). Basis für diese Berechnung bildete die Zuordnung der Eingangswahl nach Wohnort. Dabei wurde auch berücksichtigt, dass EinwohnerInnen aus Wien 22 Eingänge bei Groß-Enzersdorf benutzten, bzw. EinwohnerInnen aus Groß-Enzersdorf die Eingänge bei Aspern und Eßling nutzten. Es wurde weiters angenommen, dass die südlichen Eingänge keine nennenswerten Besucherzahlenzuwächse erfahren werden, da die städtebauliche Entwicklung nördlich der Lobau stattfindet. 


\begin{tabular}{|c|c|c|c|c|c|c|}
\hline Spitzentag & $\begin{array}{c}\text { Erhebung: } \\
1999\end{array}$ & $\begin{array}{c}* * \text { Prognose: } \\
\text { neue Besuche } \\
\text { aus Wien }\end{array}$ & $\begin{array}{l}\text { Prognose: } \\
\text { neue } \\
\text { Besuche } \\
\text { aus Wien }\end{array}$ & $\begin{array}{c}* * \text { Prognose: } \\
\text { neue } \\
\text { Besuche aus } \\
\text { Groß-Enz. }\end{array}$ & $\begin{array}{l}\text { Prognose: } \\
\text { neue } \\
\text { Besuche aus } \\
\text { Groß-Enz. }\end{array}$ & $\begin{array}{c}* * * \text { Prog- } \\
\text { nose: } \\
\text { Gesamt } \\
2025\end{array}$ \\
\hline Mühlleiten & 552 & $5 \%$ & 252 & $24 \%$ & 812 & 1.616 \\
\hline Eßlinger Furt & 1.472 & $16 \%$ & 807 & $3 \%$ & 101 & 2.380 \\
\hline Dechantweg & 566 & $6 \%$ & 303 & $3 \%$ & 101 & 970 \\
\hline Uferhaus * & 838 & $12 \%$ & 586 & $31 \%$ & 1.055 & 2.479 \\
\hline Saltenstraße & 602 & $51 \%$ & 2.572 & $11 \%$ & 372 & 3.546 \\
\hline Hubertusdamm & 576 & - & - & - & - & 576 \\
\hline Bahnhof Lobau & 206 & - & - & - & - & 206 \\
\hline Egerer Brücke * & 330 & $5 \%$ & 230 & $12 \%$ & 414 & 974 \\
\hline Kasernenbrücke* & 318 & $4 \%$ & 226 & $12 \%$ & 406 & 950 \\
\hline Luitpold Stern & 340 & - & - & - & - & 340 \\
\hline Panozzalacke & 528 & - & - & - & - & 528 \\
\hline \multirow[t]{2}{*}{ Stadler Furt * } & 96 & $1 \%$ & 68 & $4 \%$ & 122 & 286 \\
\hline & 6.424 & $100 \%$ & 5.043 & $100 \%$ & 3383 & 14.850 \\
\hline \multicolumn{7}{|l|}{ Werktag } \\
\hline Mühlleiten & 30 & $5 \%$ & 67 & $24 \%$ & 217 & 315 \\
\hline Eßlinger Furt & 189 & $16 \%$ & 216 & $3 \%$ & 27 & 432 \\
\hline Dechantweg & 187 & $6 \%$ & 81 & $3 \%$ & 27 & 295 \\
\hline Uferhaus * & 161 & $12 \%$ & 120 & $31 \%$ & 215 & 496 \\
\hline Saltenstraße & 189 & $51 \%$ & 688 & $11 \%$ & 100 & 977 \\
\hline Hubertusdamm & 243 & - & - & - & - & 243 \\
\hline Bahnhof Lobau & 228 & - & - & - & - & 228 \\
\hline Egerer Brücke * & 77 & $5 \%$ & 57 & $12 \%$ & 103 & 237 \\
\hline Kasernenbrücke* & 111 & $4 \%$ & 83 & $12 \%$ & 149 & 343 \\
\hline Luitpold Stern & 153 & - & - & - & - & 153 \\
\hline Panozzalacke & 101 & - & - & - & - & 101 \\
\hline \multirow{2}{*}{ Stadler Furt * } & 50 & $1 \%$ & 37 & $4 \%$ & 66 & 153 \\
\hline & 1.719 & $100 \%$ & 1.349 & $100 \%$ & 905 & 3.974 \\
\hline \multicolumn{7}{|c|}{$\begin{array}{l}\text { *Uferhaus }+ \text { Egerer Br }+ \text { Kasernenbr }+ \text { Stadler Furt }=22 \% \text { neue Besuche aus Wien; } 59 \% \text { neue Besuche aus } \\
\text { Groß-Enzersdorf. } \\
\text { **Anteile der neuen Besuche pro Eingang wurden der Erreichbarkeitsanalyse entnommen } \\
\text { ***Prognose (gesamt) für } 2025=\text { Erhebung } 1999+\text { neue Besuche aus Wien + neue Besuche aus Gross- } \\
\text { Enzersdorf }\end{array}$} \\
\hline
\end{tabular}

Tab. 22: Besucheraufkommen an einem Spitzen- und einem Werktag: beobachtete Besucherzahlen im Jahr 1999 und die Prognose für 2025 für zwölf Lobaueingänge.

Abb. 54 und Abb. 55 veranschaulichen den Vergleich der beobachteten Besucherzahlen (1999) und der Prognose für das Jahr 2025. In der Karte sowie im Balkendiagramm ist ersichtlich, dass die stärksten Zunahmen an BesucherInnen in der Saltenstrasse und im Eingangsbereich Uferhaus zu erwarten sind. Die südlichen Eingänge bleiben praktisch unverändert. 


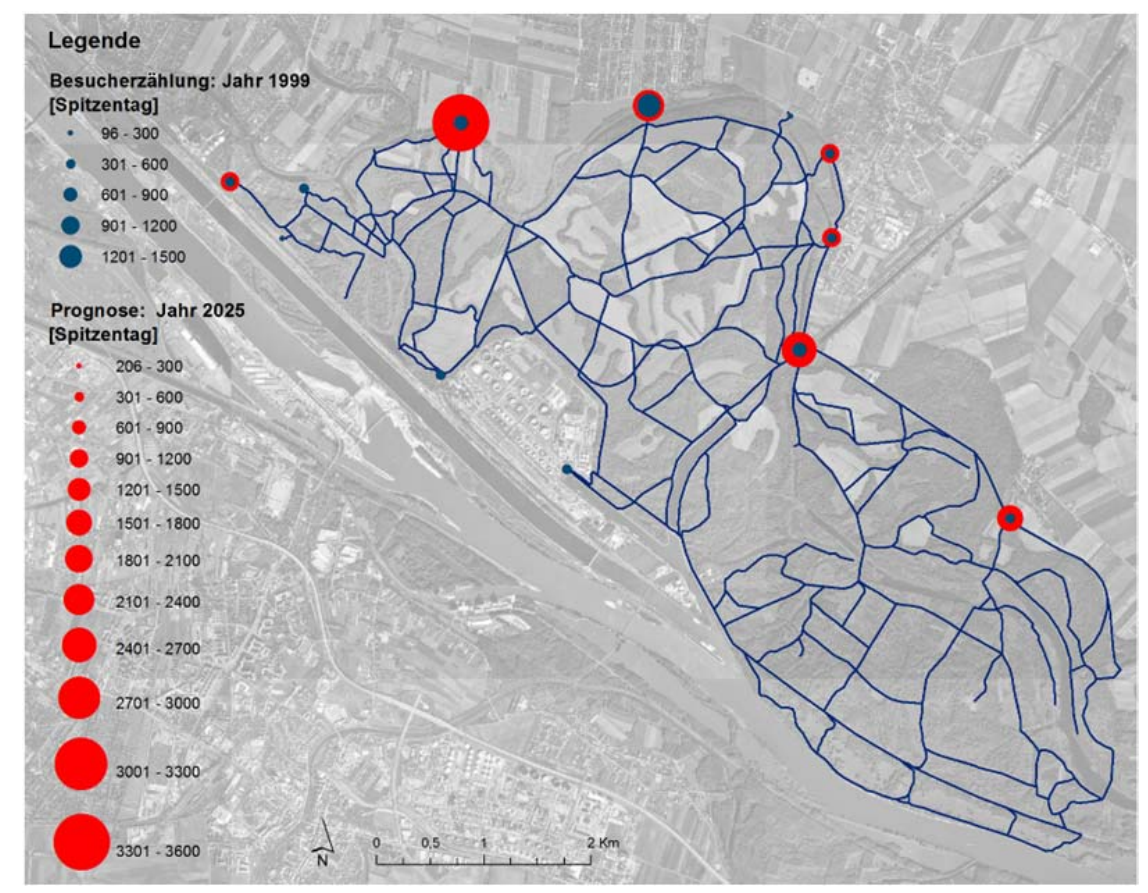

A)

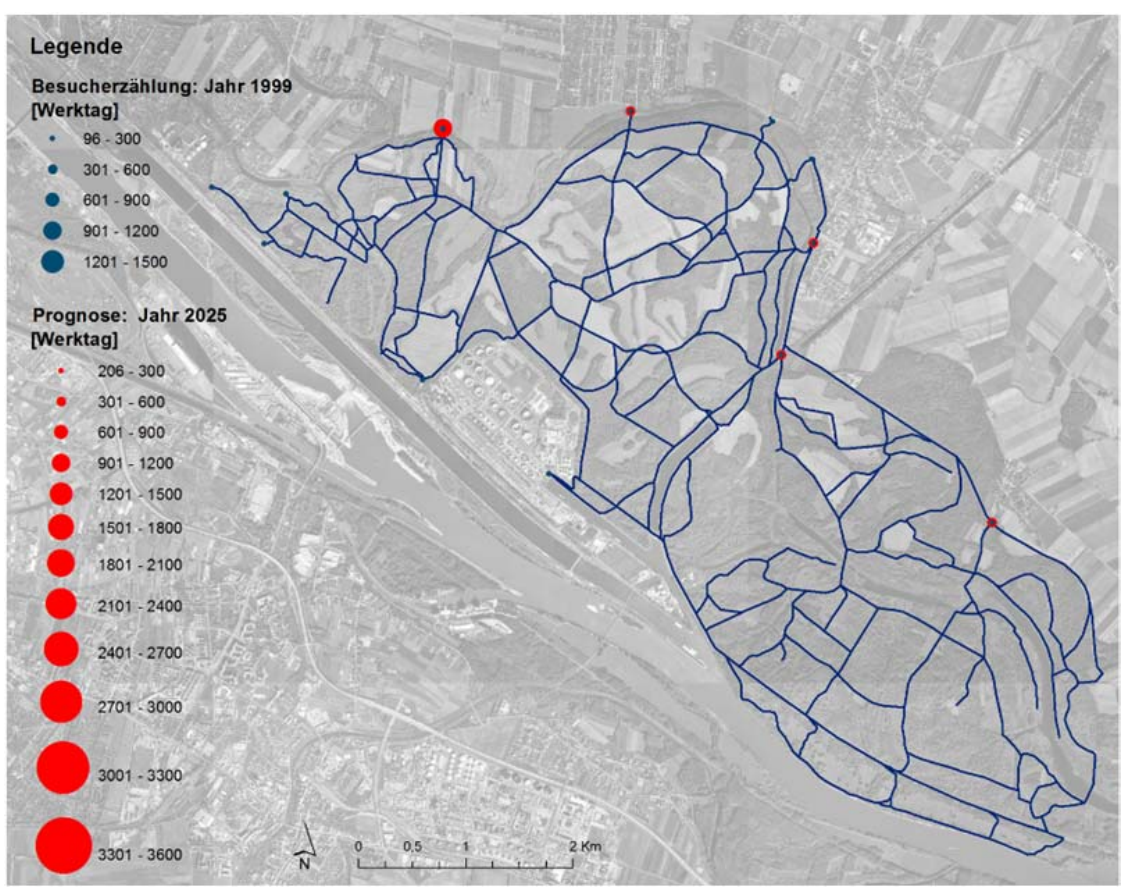

B)

Abb. 54: Vergleich der beobachteten Besucherzahlen (1999) und der Prognose für das Jahr 2025 (kartographische Darstellung); A) Besucherzahlen an einem Spitzentag; B) Besucherzahlen an einem Werktag 


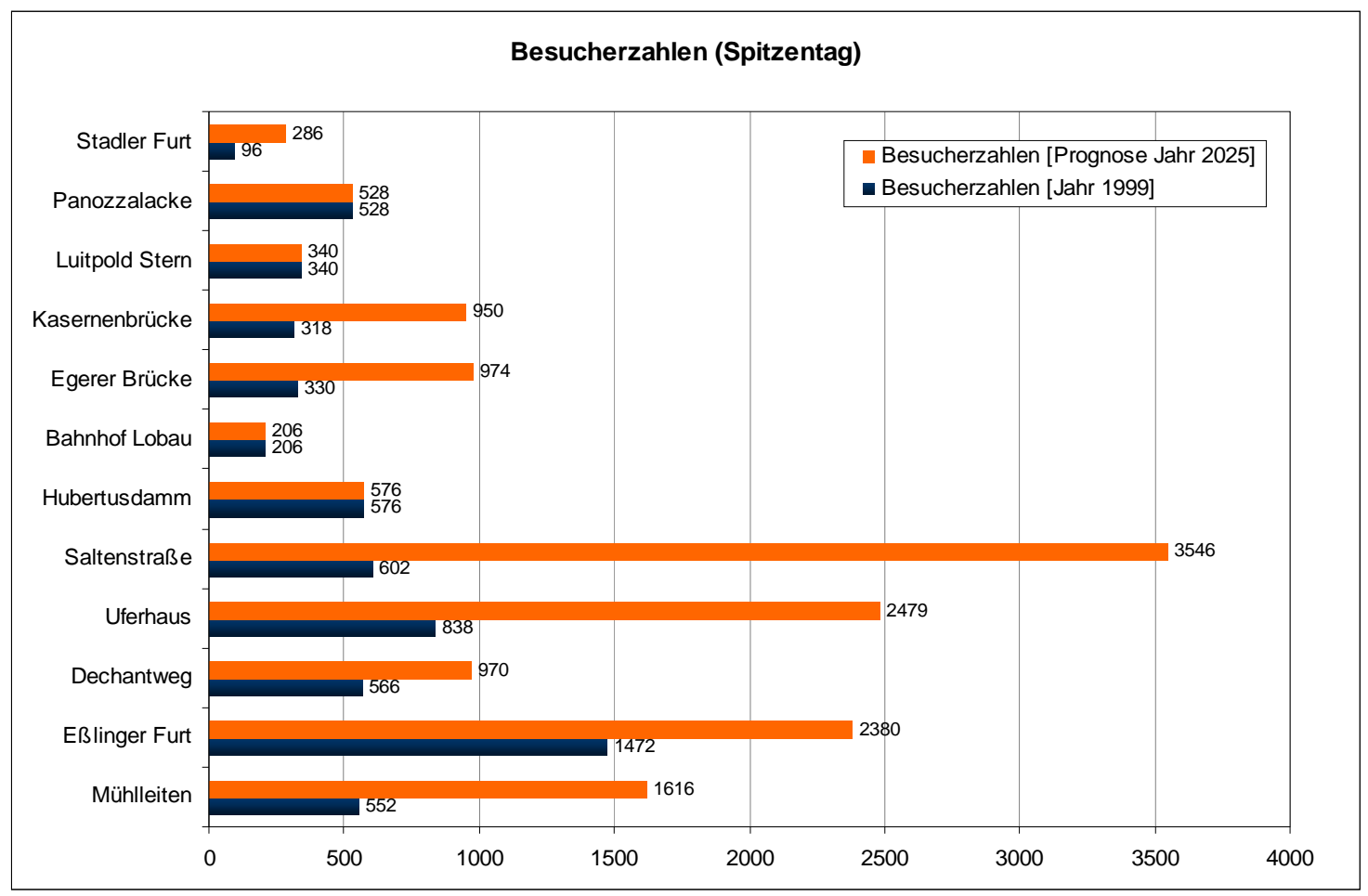

A)

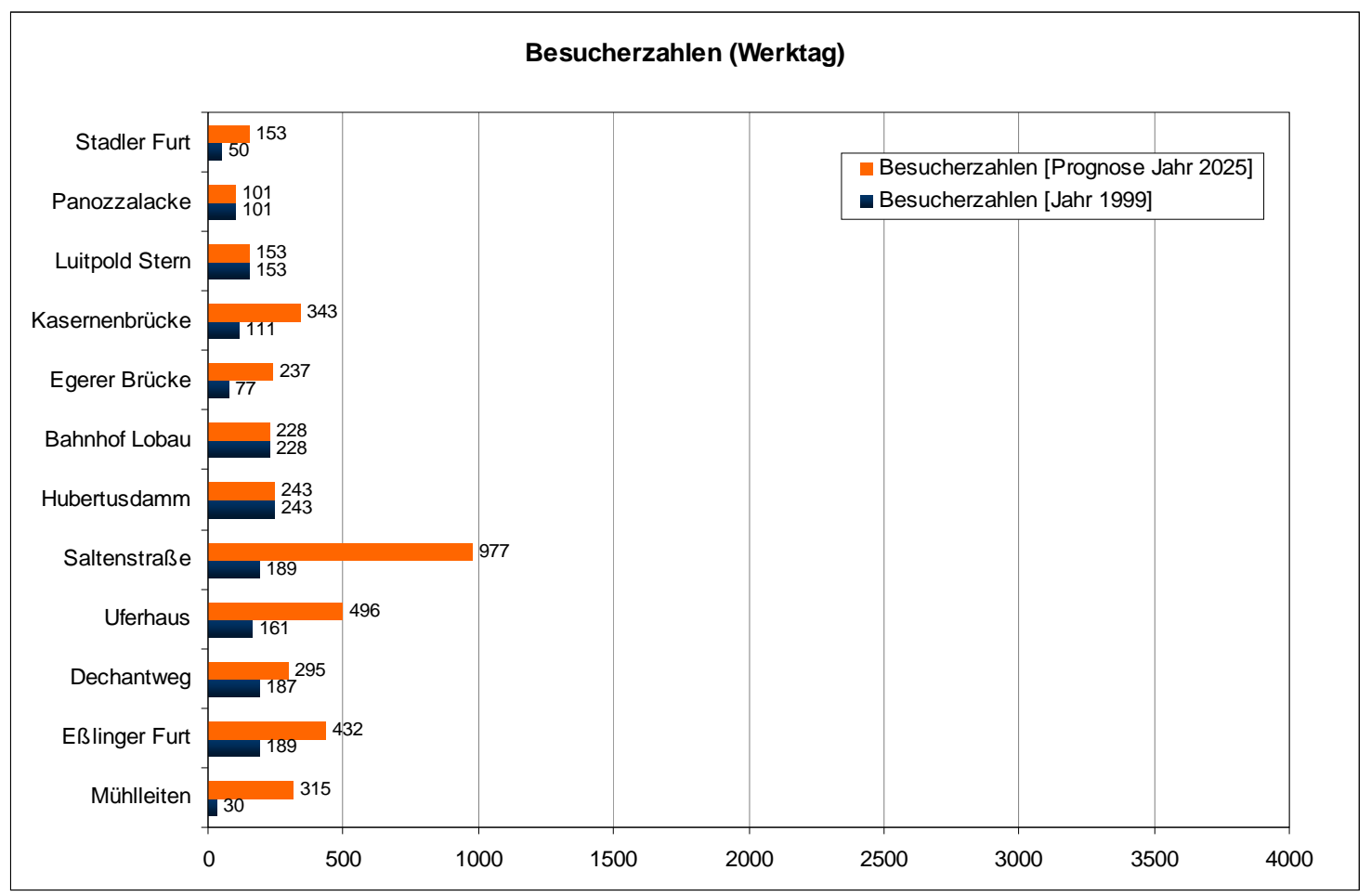

B)

Abb. 55: Vergleich der beobachteten Besucherzahlen (1999) und der Prognose für das Jahr 2025 (Balkendiagramm). Inputdaten für die MASOOR Simulation. A) Besucherzahlen an einem Spitzentag; B) Besucherzahlen an einem Werktag 


\section{Ergebnisse der räumlichen Simulation}

Für jedes Szenario wurden jeweils 5 Simulationsgänge $=$ „5 runs“ durchgeführt. Tab. 23 gibt einen Überblick über die simulierten Managementszenarien. Die Ergebnisse der MASOOR Simulation wurden in ein GIS-System integriert, um die Visualisierung der räumlichen Verteilung der BesucherInnen zu ermöglichen. Erste Vergleiche mit behavioralen Daten (erfasste Routen der Befragten am Befragungstag anhand einer Karte) zeigten dabei eine gute Übereinstimmung. Die Simulationen erfolgten für einen Spitzenbesuchstag und für einen durchschnittlichen Werktag (basierend auf den Erhebungen von 1999; Arnberger et al., 2000). Die Aussagen beziehen sich immer auf die Vegetationsperiode. Die Resultate der Simulationen wurden nachfolgend kartografisch dargestellt und zeigen die räumliche Verteilung der BesucherInnen in der Lobau (Abb. 56 bis Abb. 69).

\begin{tabular}{|c|l|c|c|}
\hline \multirow{2}{*}{ Nummer } & \multirow{2}{*}{ Szenario } & \multicolumn{2}{|c|}{ Besucherzahl } \\
\cline { 3 - 4 } & & Jahr & Spitzentag/Werktag \\
\hline 01 & Lobau A & 1999 & Spitzentag \\
\hline 02 & Lobau A & 1999 & Werktag \\
\hline 03 & Lobau A & 2025 & Spitzentag \\
\hline 04 & Lobau A & 2025 & Werktag \\
\hline 05 & Lobau B & 2025 & Spitzentag \\
\hline 06 & Lobau B & 2025 & Werktag \\
\hline 07 & Lobau A + Vorland A & 2025 & Spitzentag \\
\hline 08 & Lobau A + Vorland A & 2025 & Werktag \\
\hline 09 & Lobau A + Vorland B & 2025 & Spitzentag \\
\hline 10 & Lobau A + Vorland B & 2025 & Werktag \\
\hline 11 & Lobau B + Vorland A & 2025 & Spitzentag \\
\hline 12 & Lobau B + Vorland A & 2025 & Werktag \\
\hline 13 & Lobau B + Vorland B & 2025 & Spitzentag \\
\hline 14 & Lobau B + Vorland B & 2025 & Werktag \\
\hline
\end{tabular}

Tab. 23: Aufstellung der simulierten Managementszenarien: Lobau A = Lobau ohne Änderung; Lobau B = Lobau (GewässerSzenario „OPTIMA Lobau 1b"); Vorland A = lineare Ausgestaltung des Lobau-Vorlandes; Vorland $B=$ flächige Ausgestaltung des Lobau-Vorlandes; Lobau A bedeutet ohne, Lobau B mit Gewässerszenario 1b.

Vorland A beschreibt eine lineare Ausgestaltung des Lobau-Vorlandes. Darunter ist die Anlage eines Radwegenetzes (z.T. auf best. Straßen, die für den motorisierten Verkehr (außer AnwohnerInnen) zu sperren sind) zu verstehen. Diese Wege sind breit, geschottert oder asphaltiert und von einer Allee gesäumt. Dieses neue Radwegenetz soll v.a. ein Hineinfahren in die Lobau für die Radfahrenden weniger attraktiv machen. Vielmehr sollten sie motiviert werden, um die Lobau herumfahren. Einige Parkplätze werden von der Lobau weg verlegt, um den Besuchswiderstand zu erhöhen und um die Besucherlnnen zu zwingen, auch die neuen Wege des Vorlandes zu benutzen.

Vorland B beschreibt eine flächige Ausgestaltung des Lobau-Vorlandes mit einer Wald-Wiesen-Landschaft sowie das in Vorland A beschriebene Radwegenetz, welches zusätzlich durch ein Wanderwegenetz mit schmäleren Wegen, die von Alleen gesäumt sind, ergänzt wird.

\subsection{Räumliche Verteilung der BesucherInnen in der Lobau an einem Spitzenbesuchstag}

Die räumliche Verteilung der BesucherInnen an einem Spitzenbesuchstag wurde zwischen jener des Jahres 1999 mit jener des Laissez-faire -Szenarios, bei dem keine Maßnahmen getroffen wurden, und jenen der vier Szenarien verglichen. Gegenüber 1999 werden sich die Besucherströme 2025 in der gesamten Lobau erhöhen (Abb. 56 bis Abb. 62). Vor allem im Bereich der Saltenstraße sind dann sehr hohe Besuchermengen zu verzeichnen. Wird die Untere Lobau an die Donau angebunden (Gewässerszenario 1b), so wird in den nun schlechter erreichbaren Bereichen die Besuchermenge reduziert. Die im Lobau-Vorland projektierten Wege werden stark genutzt. 


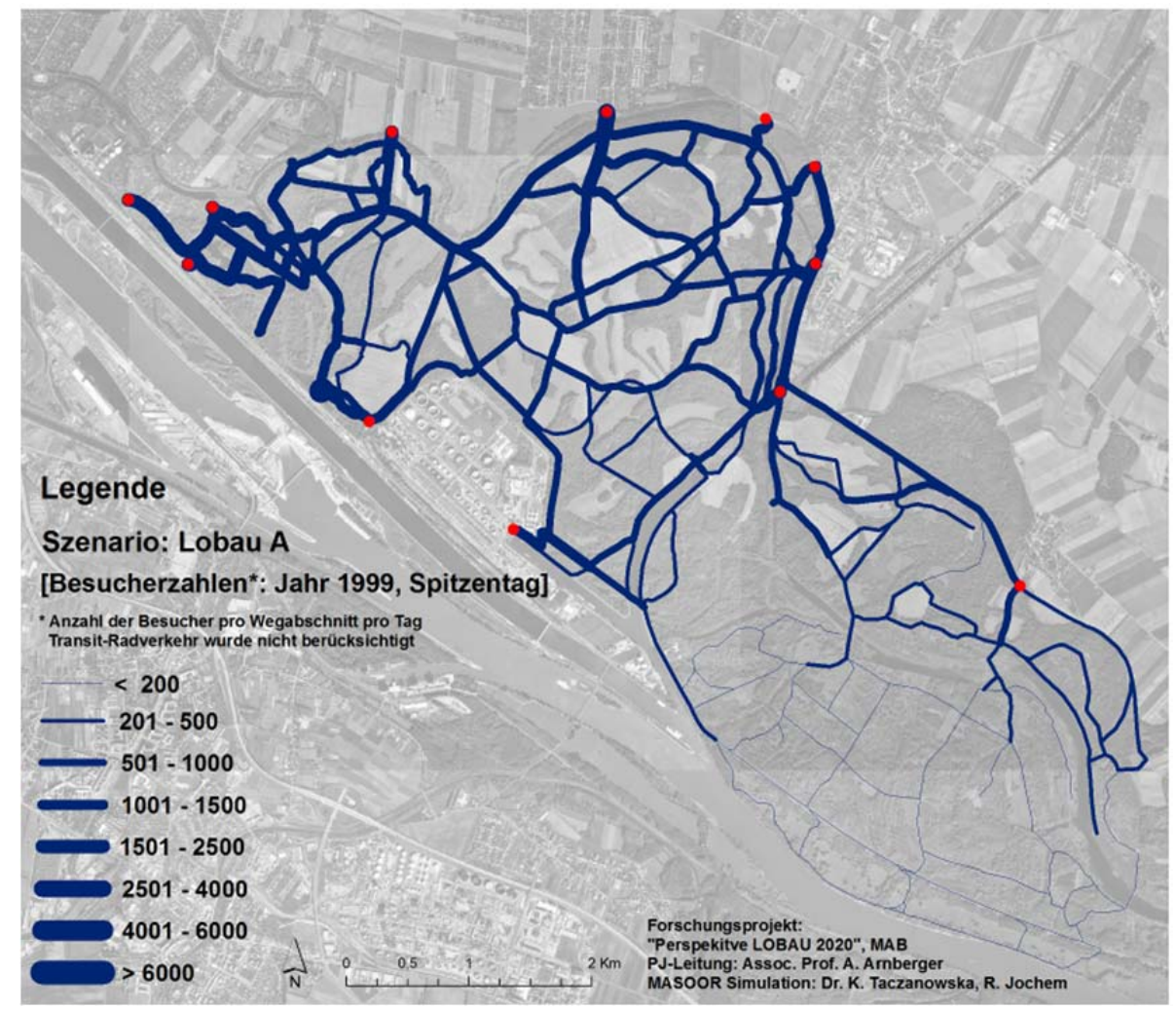

Abb. 56: Ergebnisse der MASOOR Simulation. Räumliche Verteilung der Besucherlnnen in der Lobau an einem Spitzenbesuchstag für das Jahr 1999 - Szenario-Nr. 01 (Bestand).

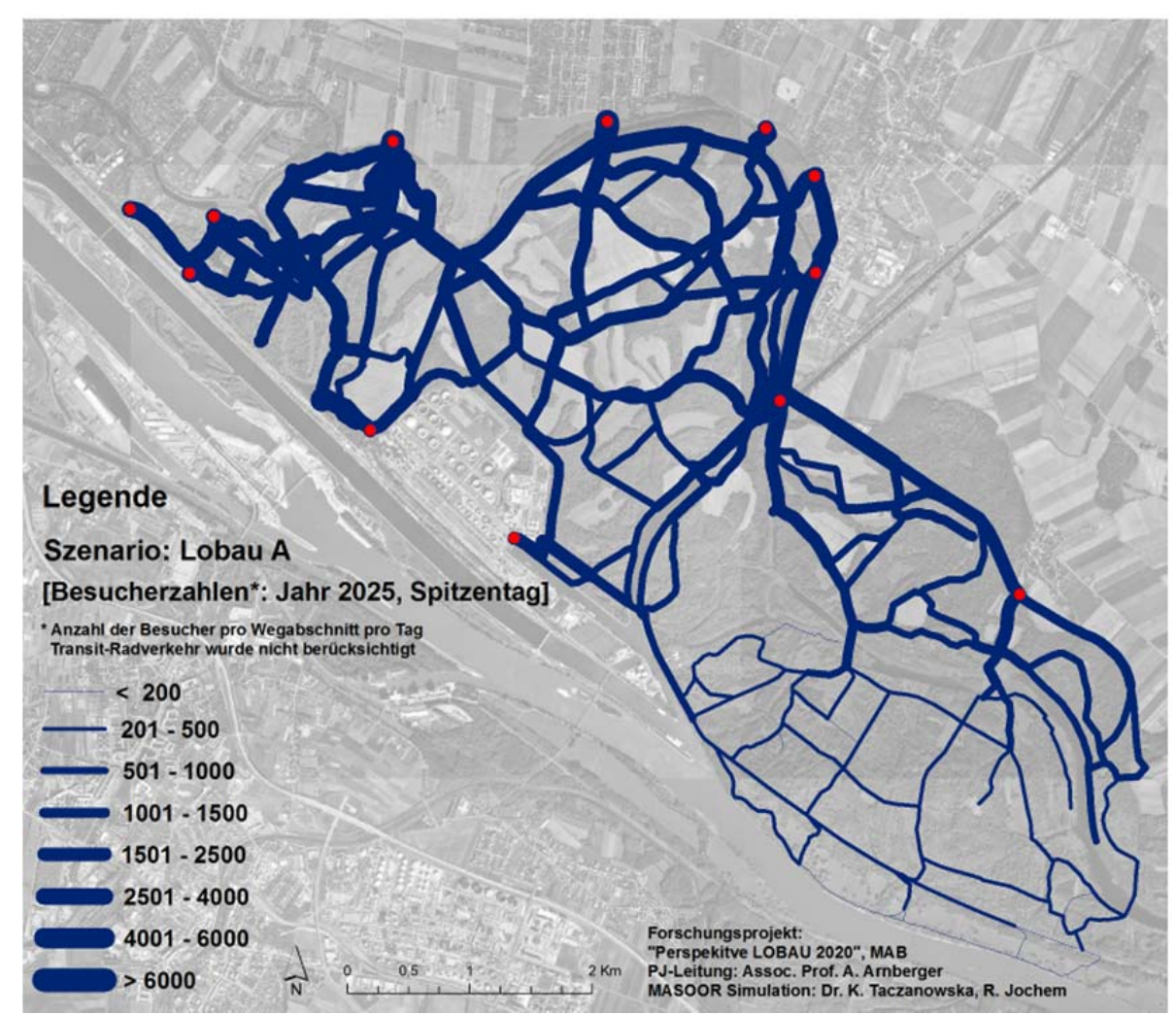

Abb. 57: Ergebnisse der MASOOR Simulation. Räumliche Verteilung der Besucherlnnen in der Lobau an einem Spitzenbesuchstag für das Jahr 2025 - Szenario-Nr. 03 („Laissez-faire -Szenario“; keine Maßnahmen getroffen). 


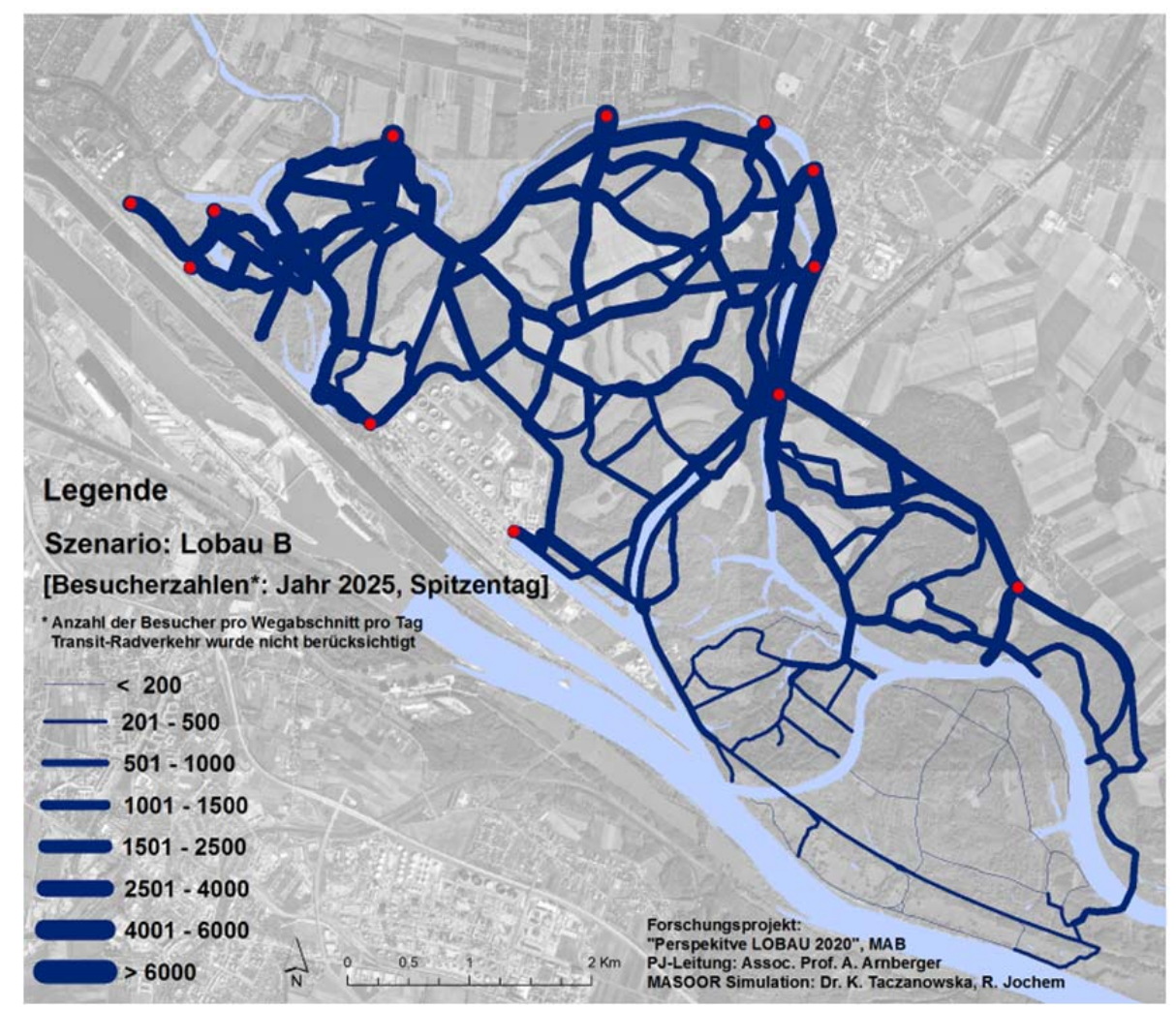

Abb. 58: Ergebnisse der MASOOR Simulation. Räumliche Verteilung der Besucherlnnen in der Lobau an einem Spitzenbesuchstag für das Jahr 2025 - Szenario-Nr. 05 (Gewässerszenario 1b, sonst keine Maßnahmen getroffen).

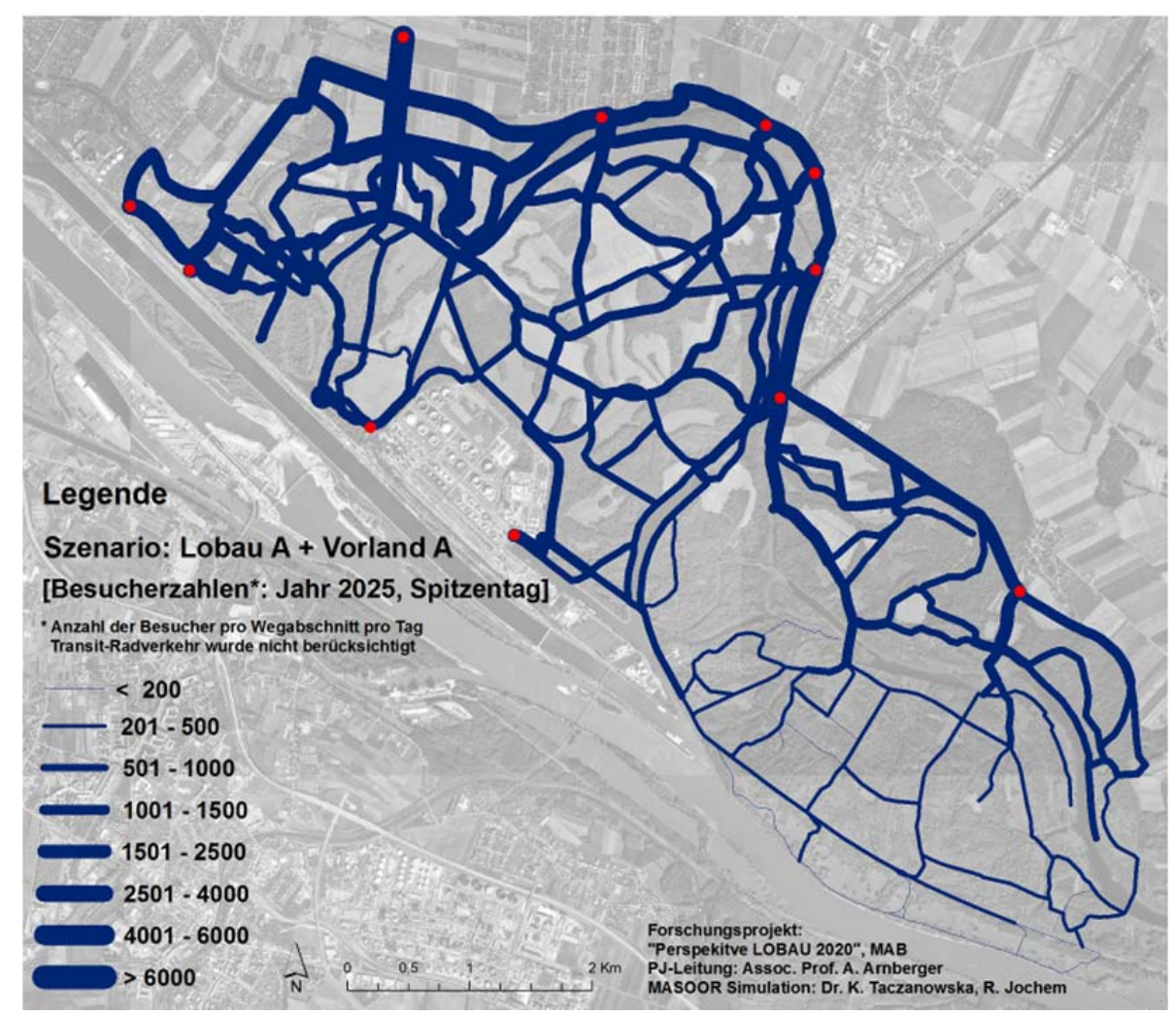

Abb. 59: Ergebnisse der MASOOR Simulation. Räumliche Verteilung der BesucherInnen in der Lobau an einem Spitzenbesuchstag für das Jahr 2025 - Szenario-Nr. 07 (lineare Ausgestaltung des Lobau-Vorlandes mit neuen Radwegen mit Alleen, Parkraumbewirtschaftung, kein Gewässerszenario 1b). 


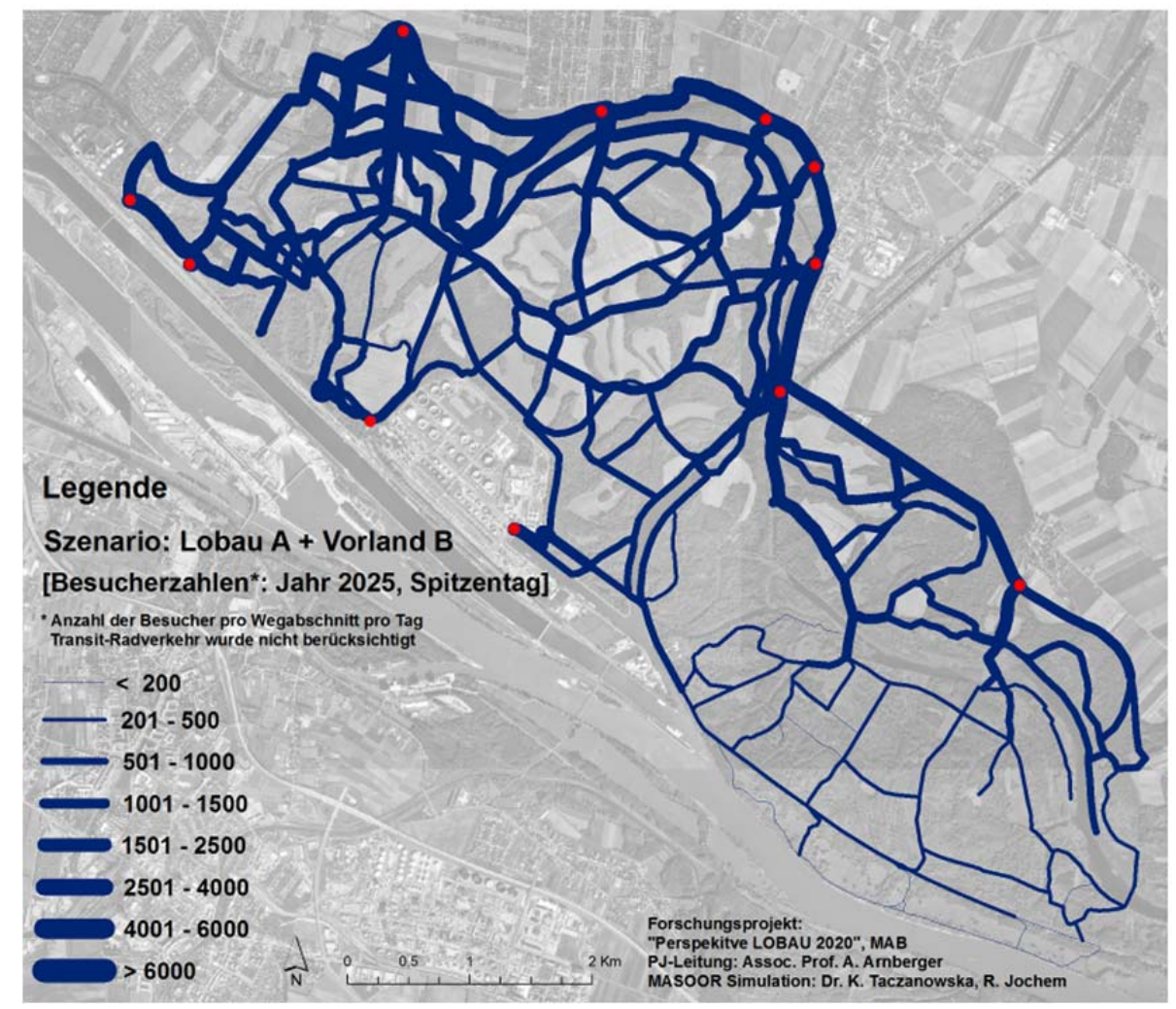

Abb. 60: Ergebnisse der MASOOR Simulation. Räumliche Verteilung der Besucherlnnen in der Lobau an einem Spitzenbesuchstag für das Jahr 2025 - Szenario-Nr. 09 (flächige Ausgestaltung des Lobau-Vorlandes mit neuen Radwegen mit Alleen, Parkraumbewirtschaftung, kein Gewässerszenario 1b).

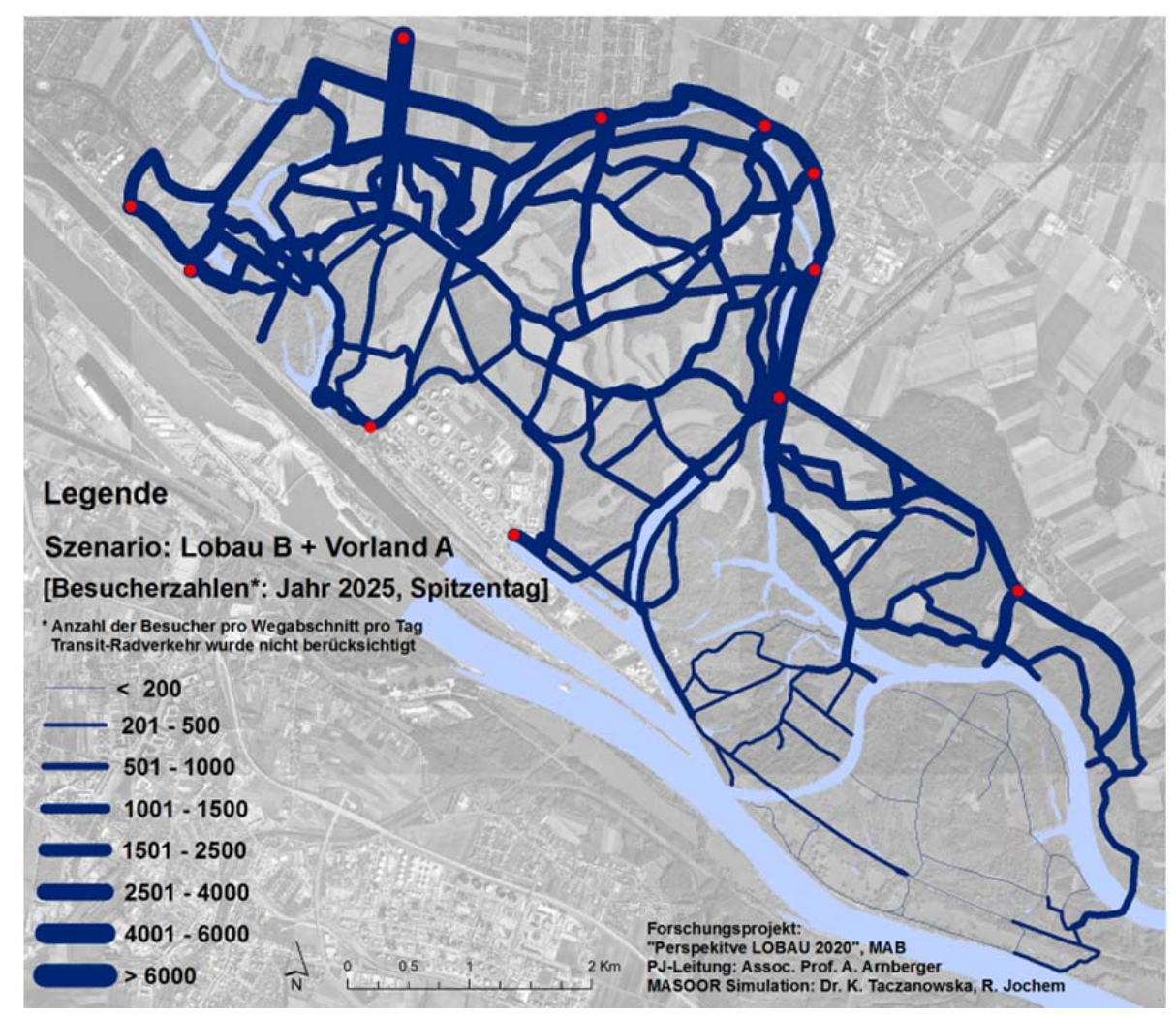

Abb. 61: Ergebnisse der MASOOR Simulation. Räumliche Verteilung der Besucherlnnen in der Lobau an einem Spitzenbesuchstag für das Jahr 2025 - Szenario-Nr. 11 (lineare Ausgestaltung des Lobau-Vorlandes mit neuen Radwegen mit Alleen, Parkraumbewirtschaftung, Gewässerszenario 1b). 


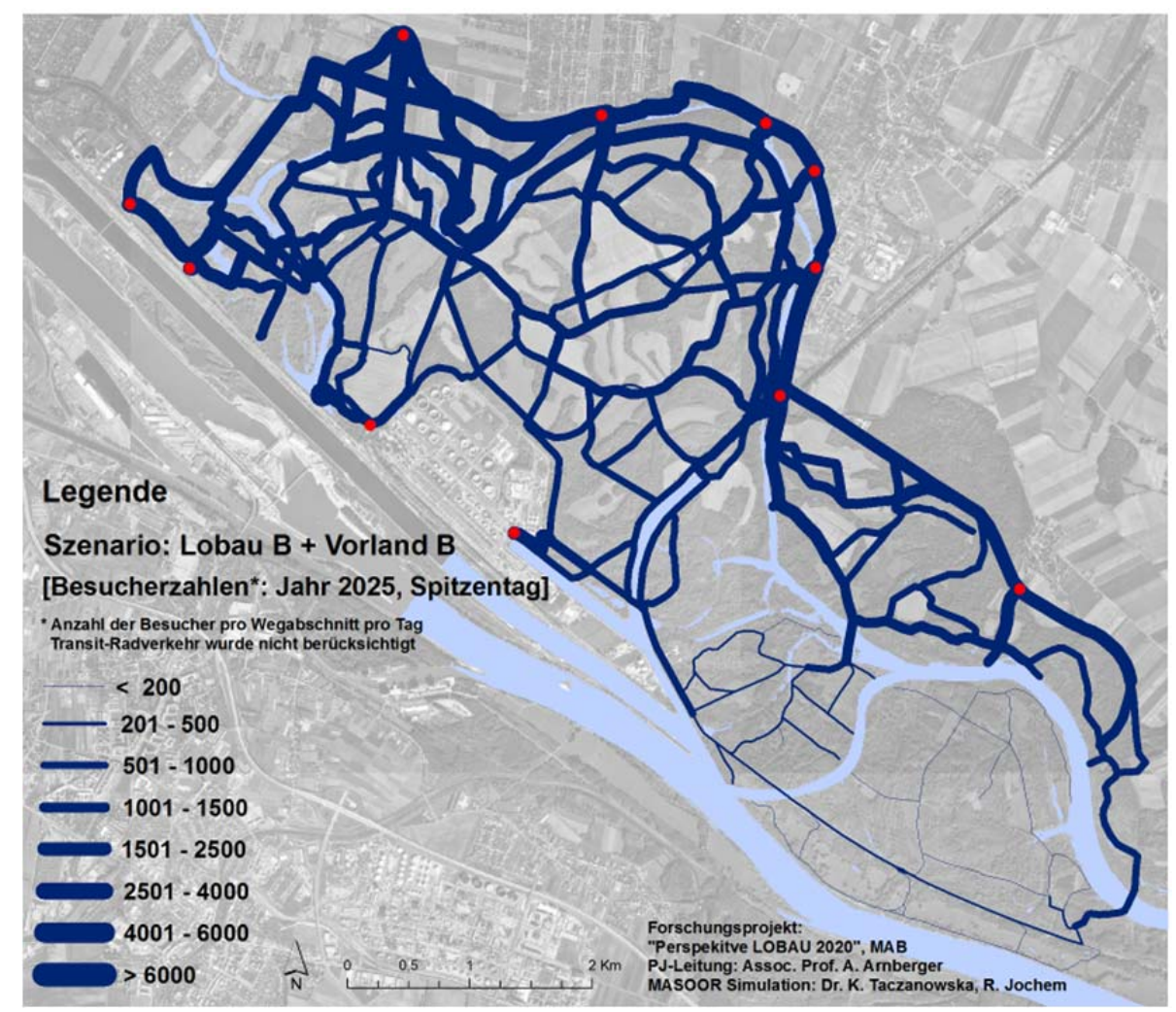

Abb. 62: Ergebnisse der MASOOR Simulation. Räumliche Verteilung der Besucherlnnen in der Lobau an einem Spitzenbesuchstag für das Jahr 2025 - Szenario-Nr. 13 (flächige Ausgestaltung des Lobau-Vorlandes mit neuen Radwegen mit Alleen, Parkraumbewirtschaftung, Gewässerszenario 1b).

\subsection{Räumliche Verteilung der BesucherInnen in der Lobau an einem Werktag}

Ähnlich zu den Ergebnissen der Besucherverteilung an einem Spitzentag zeigen sich die Ergebnisse für die Werktage (Abb. 63 bis Abb. 69). Erhöhte Besucherströme sind nun auch in den zentralen Bereichen der Oberen und Unteren Lobau zu verzeichnen. Die Auswirkungen des Gewässerszenarios $1 \mathrm{~b}$ auf die Verteilung der Besucherströme scheinen nicht so effektiv zu sein wie an dem Spitzentag, da hier kaum erhöhte Besucherströme feststellbar waren. Die im Lobau-Vorland projektierten Wege werden auch an Werktagen stark genutzt. 


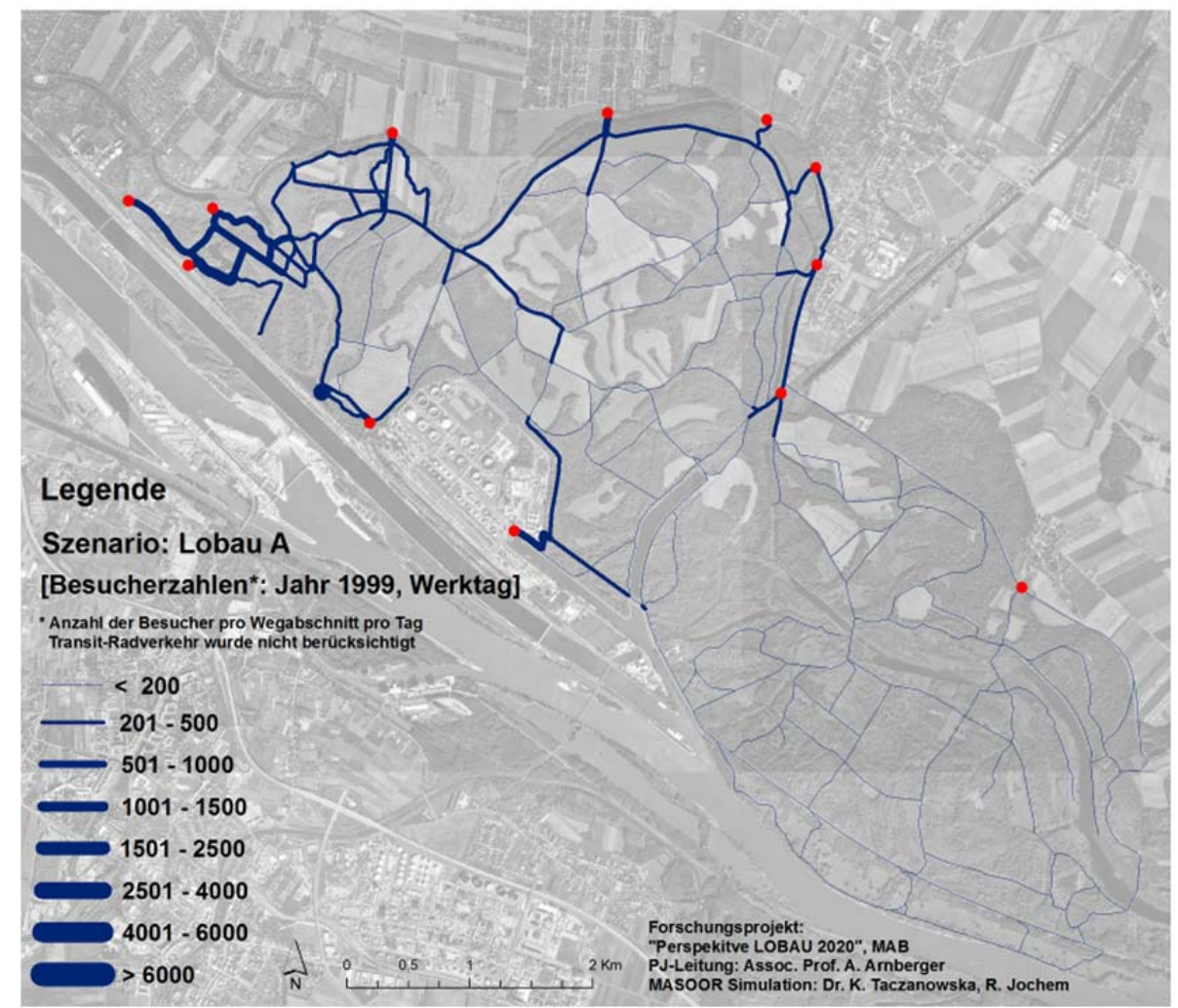

Abb. 63: Ergebnisse der MASOOR Simulation. Räumliche Verteilung der Besucherlnnen in der Lobau an einem durchschnittlichen Werktag für das Jahr 1999 - Szenario-Nr. 02 (Bestand)

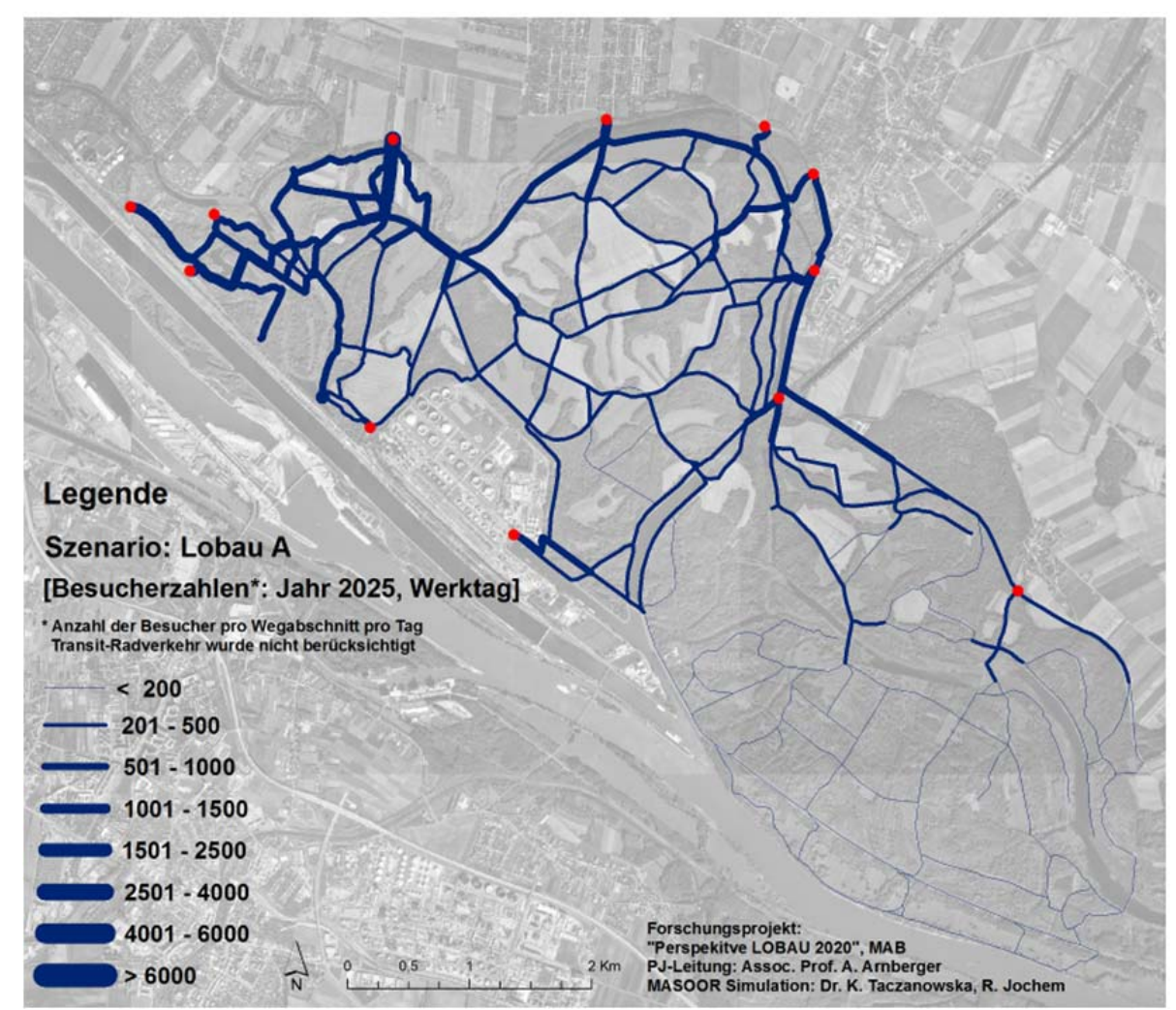

Abb. 64: Ergebnisse der MASOOR Simulation. Räumliche Verteilung der Besucherlnnen in der Lobau an einem durchschnittlichen Werktag für das Jahr 2025 - Szenario-Nr. 04 („Laissez-faire -Szenario“; keine Maßnahmen getroffen). 


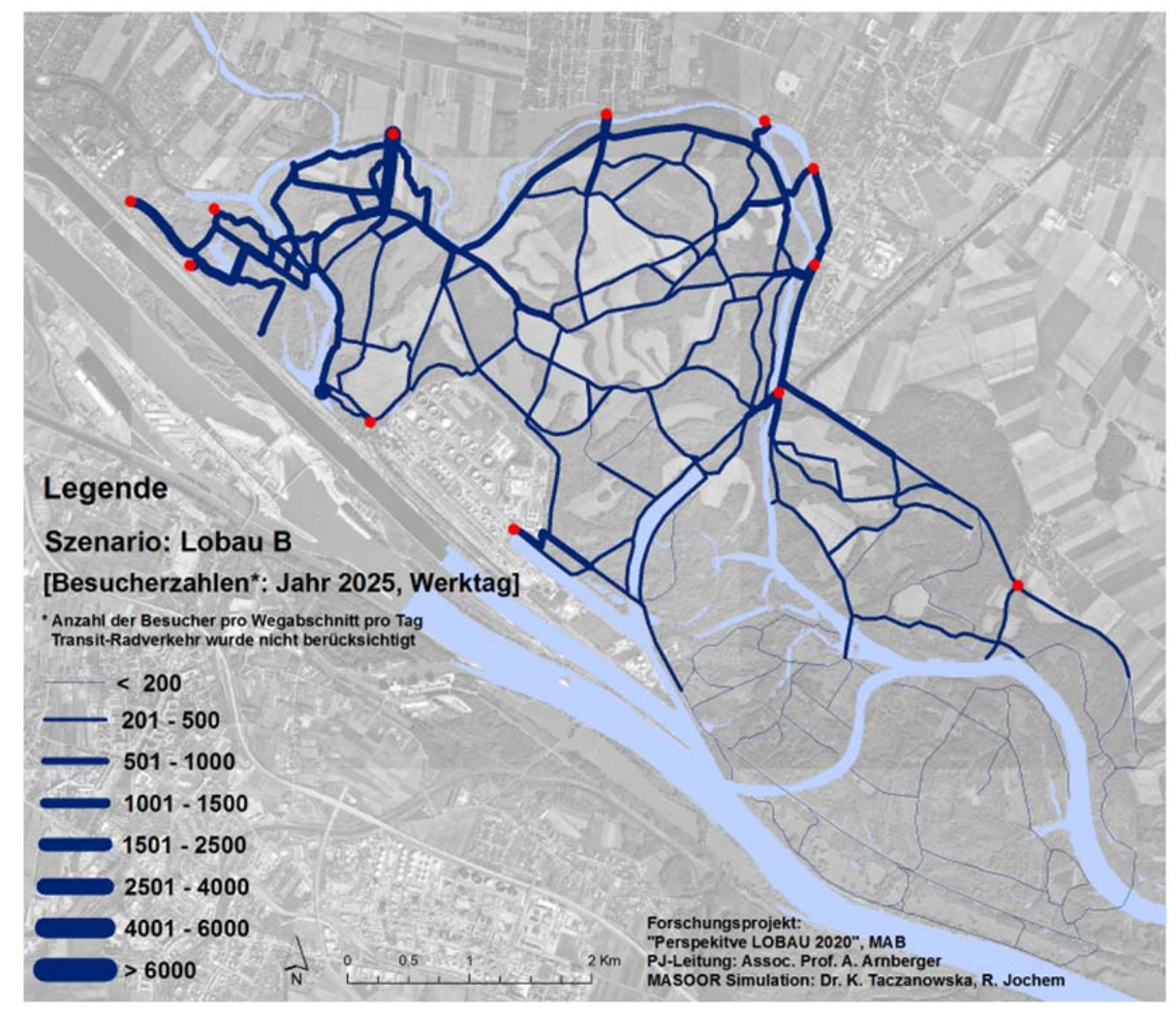

Abb. 65: Ergebnisse der MASOOR Simulation. Räumliche Verteilung der Besucherlnnen in der Lobau an einem durchschnittlichen Werktag für das Jahr 2025 - Szenario-Nr. 06 (Gewässerszenario 1b, sonst keine Maßnahmen).

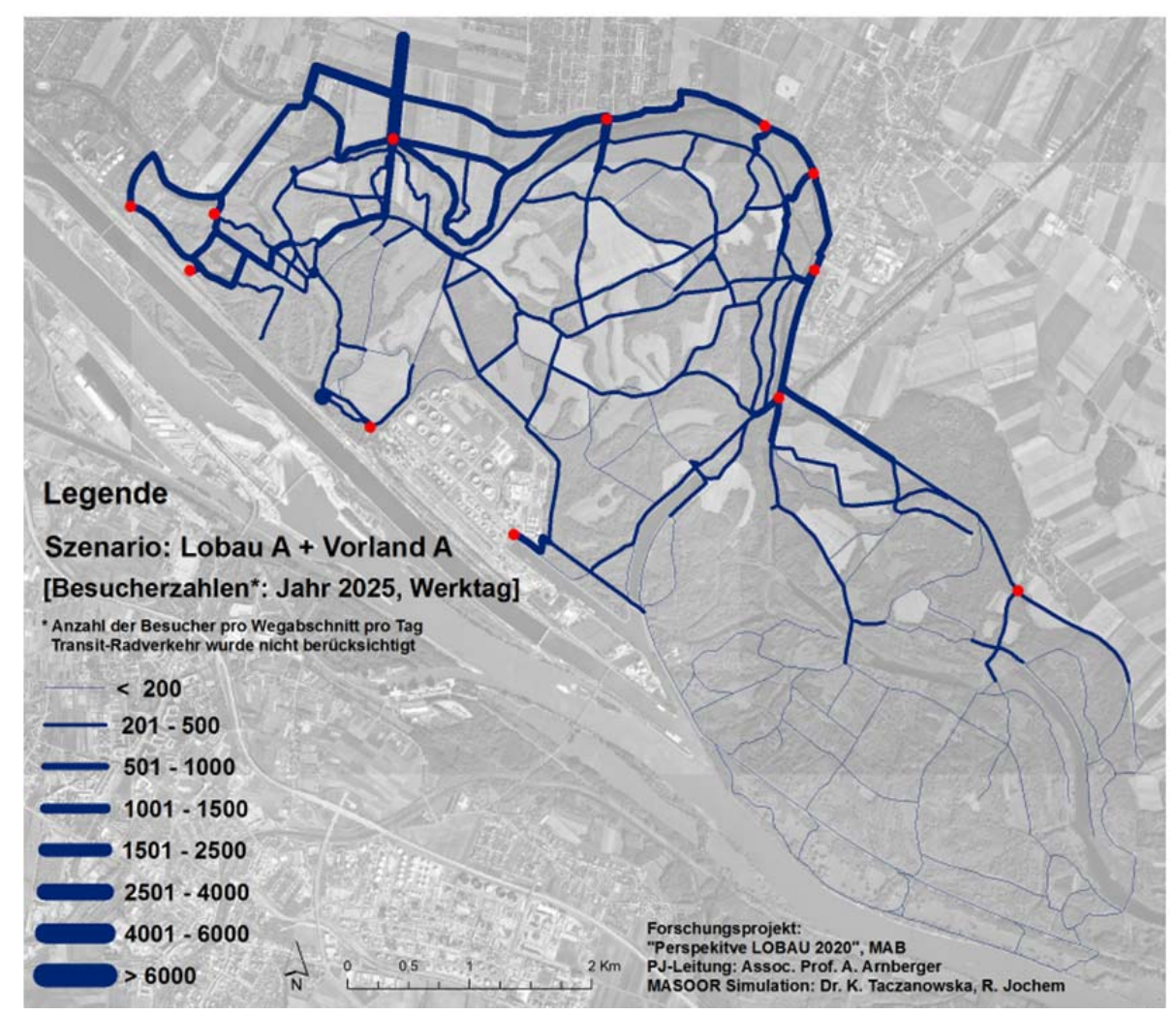

Abb. 66: Ergebnisse der MASOOR Simulation. Räumliche Verteilung der BesucherInnen in der Lobau an einem durchschnittlichen Werktag für das Jahr 2025 - Szenario-Nr. 08 (lineare Ausgestaltung des Lobau-Vorlandes mit neuen Radwegen mit Alleen, Parkraumbewirtschaftung, kein Gewässerszenario 1b). 


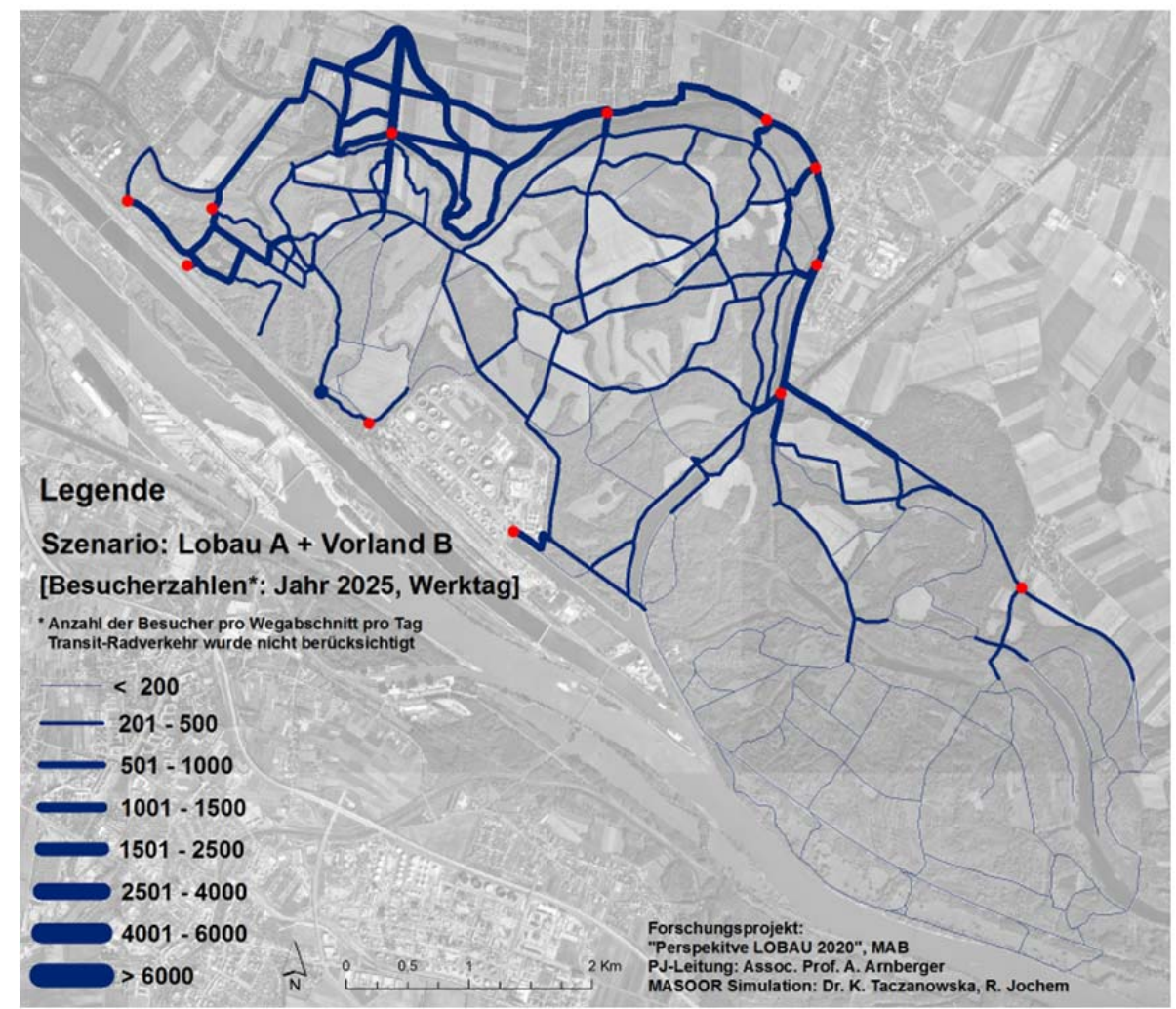

Abb. 67: Ergebnisse der MASOOR Simulation. Räumliche Verteilung der Besucherlnnen in der Lobau an einem durchschnittlichen Werktag für das Jahr 2025 - Szenario-Nr. 10 (flächige Ausgestaltung des Lobau-Vorlandes mit neuen Radwegen mit Alleen, Parkraumbewirtschaftung, kein Gewässerszenario 1b).

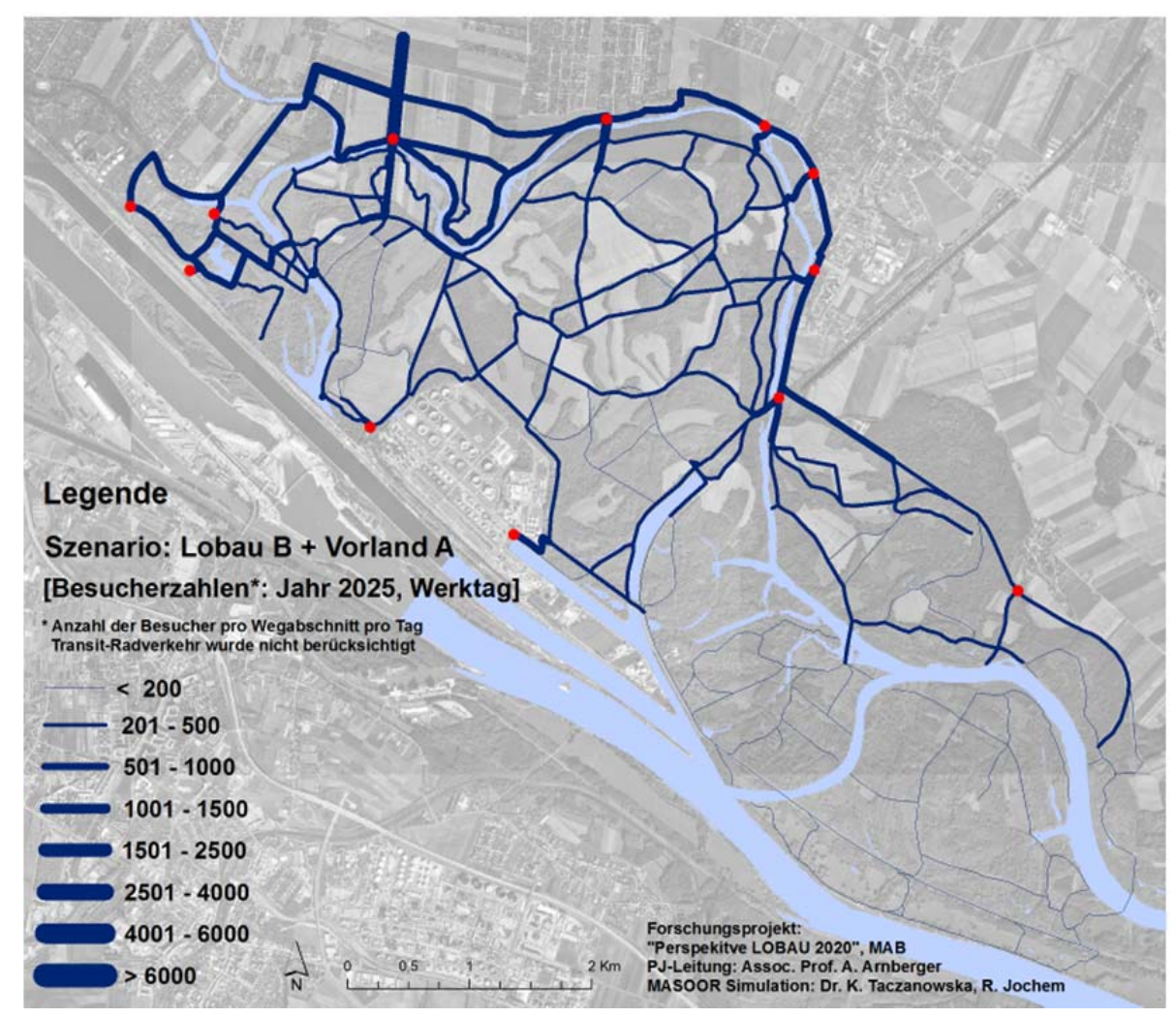

Abb. 68: Ergebnisse der MASOOR Simulation. Räumliche Verteilung der Besucherlnnen in der Lobau an einem durchschnittlichen Werktag für das Jahr 2025 - Szenario-Nr. 12 (lineare Ausgestaltung des Lobau-Vorlandes mit neuen Radwegen mit Alleen, Parkraumbewirtschaftung, Gewässerszenario 1b). 


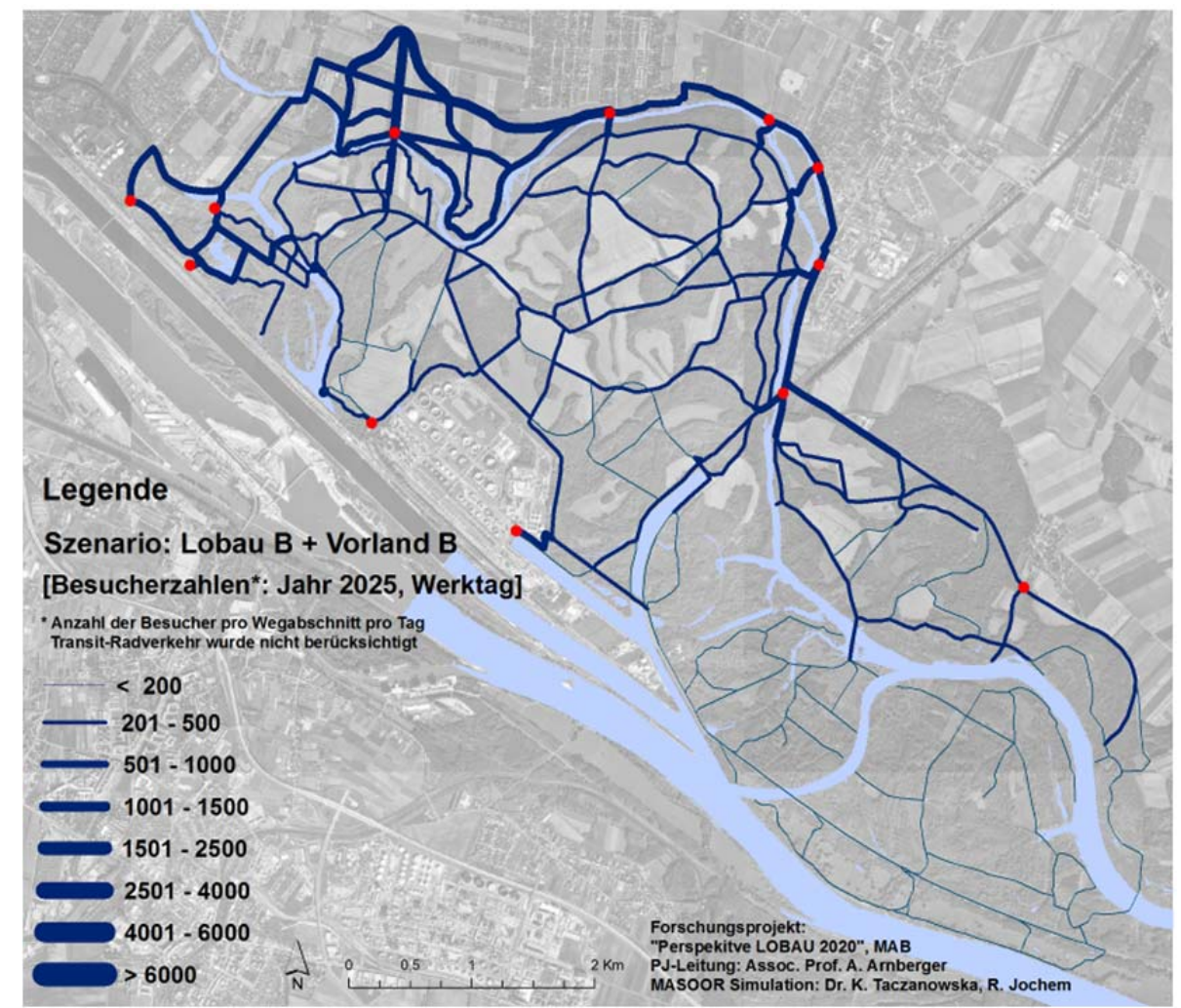

Abb. 69: Ergebnisse der MASOOR Simulation. Räumliche Verteilung der Besucherlnnen in der Lobau an einem durchschnittlichen Werktag für das Jahr 2025 - Szenario-Nr. 14 (flächige Ausgestaltung des Lobau-Vorlandes mit neuen Radwegen mit Alleen, Parkraumbewirtschaftung, Gewässerszenario 1b). 


\section{Bewertung der Szenarien}

Als Basis für die Bewertung der modellierten Szenarien wurden Kriterien und Indikatoren definiert. Diese stammen aus den Bereichen der Erholungsplanung, Stadt- und Verkehrsplanung, Wildtierökologie und der Ökologie von aquatischen und terrestrischen Systemen. Die Kriterien wurden aus den Auswertungen und Modellierungen der einzelnen Arbeitsgruppen abgeleitet und werden flächenbasiert dargestellt. Mit Hilfe der Kriterien konnten die Auswirkungen der einzelnen Szenarien in Hinblick auf Erholungssuchende, Stadt- und Verkehrsplanung und den Naturraum bewertet (Risikoanalyse) und verglichen werden.

Jeder Fachbereich definierte als Basis für die rasterbasierte Risikoanalyse Indikatoren zur Bewertung der modellierten Szenarien, um die nachhaltigsten Maßnahmenszenarien zu identifizieren. Die rasterbasierte Risikoanalyse wurde nur auf die Lobau und nicht auf das Lobau-Vorland angewendet. Sie basierte auf einem Basis-Raster von 25 x $25 \mathrm{~m}\left(625 \mathrm{~m}^{2}\right)$. Zirka 37.000 Rasterzellen wurden damit bewertet. Anhand der Indikatoren wurde die Sensibilität des Schutzgebietes anhand einer 5-stufigen Skala erfasst. Die Beeinträchtigungsintensität setzte sich aus der Eintrittswahrscheinlichkeit (Spitzentag 1 Mal, Werktage ca. 150 Mal im Jahr in der wärmeren Jahreszeit) und der Nutzungsintensität bezogen auf die prognostizierten Besucherströme des Jahres 2025 zusammen. Damit wurde das Risiko der Beeinträchtigung je Raster bewertet. Allerdings konnte nicht jeder Fachbereich die rasterbasierte Risikoanalyse für jeden Indikator anwenden.

\subsection{Bewertungskriterien aus vegetationsökologischer Sicht}

Aus vegetationsökologischer Sicht sind es zwei Faktoren, die als wesentlich für die Beurteilung des Einflusses der BesucherInnen auf die Flora und Fauna des Untersuchungsgebietes gelten - dies sind einerseits die Vegetationstypen auf synökologischer Basis („Pflanzengesellschaften“) entlang der Wege und andererseits die Darstellung des Kultureinflusses auf die Waldökosysteme.

\subsubsection{Bewertung des Einflusses einer steigenden Erholungsnutzung auf die Hemerobie}

Zur Ausarbeitung effizienter Schutzstrategien für Waldökosysteme braucht es umfassende Informationen. In diesem Zusammenhang spielen die Bewertung der Wälder hinsichtlich der Intensität der menschlichen Nutzungskultur bzw. das Erfassen des Maßes der Veränderung der natürlichen Waldökosysteme eine wesentliche Rolle. Diese Maß der menschlichen Nutzungskultur wird mit dem Begriff der Hemerobie bezeichnet (Reiter et al. 2005).

Das Hemerobiekonzept beruht auf einem aktualistischen Ansatz was bedeutet, dass die heutigen Standortsverhältnisse als Ausgangsbasis zur Analyse des menschlichen Einflusses auf ein Ökosystem herangezogen werden. Bezugsgröße der Hemerobiebewertung ist die ,potentiell natürliche Waldgesellschaft“. Darunter versteht man jene Artenzusammensetzung, welche sich unter den gegenwärtigen Umweltbedingungen und Standortsverhältnissen ausbilden würde, wenn der Mensch nicht weiter lenkend eingreift und wenn die Vegetation Zeit fände, sich bis zu ihrem Endzustand zu entwickeln. Erhebungsbasis im Gelände sind eindeutig messbare und nachvollziehbare Einzelkriterien wie z.B. „Naturnähe der Baumartenkombination“, „Naturnähe der Bodenvegetation“, „Menge und Qualität des Totholzes“, „Intensität der Nutzungen“ etc. (Grabherr et al., 1998).

Der Begriff der „Hemerobie“ leitet sich vom griechischen „hemeros“ (gezähmt, kultiviert) und „bios“ (Leben) ab (vgl. Sukopp 1969). Damit liefert dieser Begriff grundsätzlich keine Aussage über die Naturnähe, sondern über das Ausmaß des menschlichen Kultureinflusses in Ökosystemen. Für die Hemerobiebewertung ist ein aktualistischer Ansatz, der bisherige Veränderungen des Standortes mit einbezieht, notwendig. Als Bewertungsgrundlage und Nullpunkt für die Studie ist die potentiell natürliche Waldgesellschaft (PNWG), also jene Schluss- oder Klimaxvegetation, die sich aus dem heutigen Standortspotential entwickeln würde, als Soll-Größe heranzuziehen (vgl. Grabherr et al., 1998). Als PNWG bezeichnet man nach Tüxen (1956) jene Vegetation, „,...] die sich heute nach dem Aufhören des menschlichen Einflusses auf irreversibel veränderten Standorten einstellen würde.“

\subsubsection{Das Arbeitsgebiet aus vegetationsökologischer Sicht}

Einher gehend mit dem lateralen Gradienten von Habitattypen (ausgedehnte Schotterflächen bis hin zu stabilen, höher gelegenen Aubereichen) erfolgt die Differenzierung der Vegetation in 
gewässergebundene Vegetation, Pionier- und Folgegesellschaften der dynamischen Au, sowie in die Endgesellschaften der struktur- und artenreichen Hartholzmischwälder der stabilen Au. Als Sonderstandorte gelten die Heißländen, die durch ihren großen Flurabstand und die Wasserdurchläßigkeit des Schotterkörpers eine trockene, steppenartige Vegetation aufweisen. Viele der typischen Gesellschaften der Au sind auf wiederkehrende Umlagerungsvorgänge angewiesen.

Flußauen als Ökosysteme im hydrologischen und dynamischen Regime von Fließgewässern, sind in Mitteleuropa vor allem von unterschiedliche struktur- und artenreichen Auenwaldtypen und deren Anfangsstadien geprägt. Die ökologischen Rahmenbedingungen sind im allgemeinen hochanstehender, aber mit dem Flusswasserstand in Zusammenspiel oszillierender Grundwasserstand, sowie periodische Überschwemmungen mit anschließendem Trockenfallen, die zur Entwicklung einer primär hydrologisch geprägten Vegetation führen. Neben der durch Makrophyten geprägten Gewässervegetation und der Ufervegetation sind es vor allem die beiden Waldtypen - die dynamische (weiche) und die stabile (harte) $\mathrm{Au}$, die dieses Ökosystem prägen.

Die dynamische $\mathrm{Au}$ ist eine von Hochwässern regelmäßig umgelagerte flussnahe Aue mit dynamischen Weichholzauenwäldern, die von breiten Flussarmen durchzogen und von hochaufgeschütteten Grobsediment-Anlandungen durchsetzt sind. Dieser gegenüber steht die stabile $\mathrm{Au}$, die sich als eine von episodischen Hochwässern durchpulste flussfernere Aue mit altholzreichen Hartholzauwäldern definiert, die von Saumgängen durchzogen und von verlandenden Altwässern durchsetzt sind. Als Funktion unterschiedlicher Wasserstände bei ebenso unterschiedlicher Schleppkraft vollzieht sich die Standortsdynamik in hohem Maße in der dynamischen, in geringerem Maße in der stabilen Au. Als Sonderstandorte gelten die Heißländen, die durch ihren großen Flurabstand und die Wasserdurchläßigkeit des Schotterkörpers eine trockene, steppenartige Vegetation aufweisen

Mit der Erklärung der Unteren Lobau zum Vollnaturschutzgebiet (Lobauverordnung, LGBl. für Wien Nr. 32/1978) wurde im Projektgebiet ab 1978 weitgehend naturnahe Forstwirtschaft betrieben. Die früheren Bewirtschaftungsformen haben jedoch die Bestandesstruktur des Auwaldes auf großen Flächen stark verändert und so dominieren Hybridpappeln in weiten Bereichen den Bestand. Auch andere neophytischen Baumarten wurden gefördert (z.B. Robinie, Eschenahorn, Götterbaum). Diese Arten breiten sich sehr stark aus und stellen eine Gefahr für den einheimischen Artenbestand dar.

$\mathrm{Zu}$ den häufigsten Baumarten in der Lobau zählen neben der Hybridpappel noch die Esche, Silberpappel, Schwarzpappel. Baumarten der Harten Au wie z.B. Bergahorn, Robinie, Stieleiche, Linden, Ulmen und Walnuss haben zur Zeit einen etwas höheren Anteil in den Naturzonen mit Managementmaßnahmen. Die Altersklassenverteilung der Bäume zeigt ein durchschnittliches Alter von 40 Jahren.

Trotz seit Jahrhunderten andauernden Regulierungsmaßnahmen hat sich in der Unteren Lobau noch vieles in seiner ursprünglichen, geschlossenen und vielfältigen Form erhalten. Der Artenreichtum dieser Auwälder bewirkt, dass dieses Gebiet aus naturschutzfachlicher Sicht nicht hoch genug eingeschätzt werden kann. So verwundert es nicht, dass hier Nationalpark, Biosphärenreservat, Naturschutzgebiet, Ramsar-Gebiet und Natura 2000 Gebiet als Naturschutzstrategien dieses einzigartige Gebiet vor dem menschlichen Zugriff schützen.

Die Nominierung des Untersuchungsgebietes als Natura2000 Gebiet ergab sich auf Grund der Ausstattung mit folgenden Lebensraumtypen: zeitweilig Vegetation trockenfallender Ufer (FFH - Typ 3132), oligi- bis mesotrophe kalkhaltige Gewässer mit benthischer Vegetation aus Armleuchteralgen (FFH - Typ 3140), natürlich eutrophe Seen mit einer Vegetation des Magnopotamions oder Hydrocharitions (FFH - Typ 3150), alpine Flüsse mit Ufergehölze von Salix eleagnos (FFH - Typ 3240), Unterwasservegetation in Fliessgewässern der Submantstufe und der Ebene (FFH - Typ 3260), naturnahe Kalk-Trockenrasen und deren Verbuschungsstadien (FFH - Typ 6210), magere Flachland Mähwiesen (FFH - Typ 91E0), Auwälder mit Erle und Esche und Hartholzauwälder (FFH - Typ 91F0). Neben diesen FFH Lebensraumtypen gibt es zahlreiche Arten im Untersuchungsgebiet, die nach der FFH - Richtlinie (z.B. Biber, Sumpfschildkröte, Zingel, Kriech-Sellerie) bzw. nach der Vogelschutzrichtlinie (z.B. Schwarzmilan, Fischadler, Eisvogel) geschützt sind.

Aus vegetationsökologischer Sicht sind es zwei Faktoren, die als wesentlich für die Beurteilung des Einflusses der BesucherInnen auf die Flora und Fauna des Untersuchungsgebietes gelten - dies sind einerseits die Vegetationstypen auf synökologischer Basis („Pflanzengesellschaften“) entlang der Wege und andererseits die Darstellung des Kultureinflusses auf die Waldökosysteme. Im zweiten 
Projektjahr wurde von der Gruppe Vegetationsökologie der Faktor Vegetationstyp bearbeitet. Neben der Verwendung bestehender Datengrundlagen aus dem Projekt OptimaLobau wurde die gestellte Aufgabe auch durch Freilanderhebungen des Projektteams, durch Diplomarbeiten und einer Lehrveranstaltung erfüllt. Ziel der Erhebungen war es, einen Überblick über die aktuellen Vegetationsverhältnisse der Lobau zu geben und die B-Diversität dieser Aulandschaft im Bereich der Wege abzubilden. Mittels Vegetationsaufnahmen auf objektiv ausgewählten Flächen wurden Informationen $\mathrm{zu}$ den verschiedenen Vegetationstypen gesammelt, die auch zur Evaluierung der Modelle der agentenbasierten Analyse dienen (Aichhorn \& Paukovits 2010).

\subsubsection{Daten und Methoden}

Für das Bewertungsverfahren des Einflusses der Besucherfrequenz auf die Vegetation wurden flächendeckende Vegetationsmodelle überarbeitet, Karten zur Potentiellen Waldvegetation erstellt, flächendeckende Hemerobiemodelle (Kultureinfluss) zum Wald des Untersuchungsgebietes interpoliert und die aus den agentenbasierten Simulationen gewonnenen Ergebnissen zu den Besucherfrequenzen von 1999 und 2025. Die dem Bewertungsverfahren zugrunde liegende Hypothese lautet: „Bereiche hoher Hemerobie reagieren weniger sensibel auf Besucherdruck“

\section{Das Vegetationsmodell}

Für mitteleuropäische Auenökosysteme und deren terrestrische Komponenten ist diese Wissensbasis durch zahlreiche wissenschaftliche Untersuchungen weitgehend gegeben. Der schwierige Versuch, das vorhandene Wissen nicht nur systematisch zusammenzustellen, sondern auch zu operationalisieren und damit zu einer Ausgangsbasis für prediktive Modellbildung und Eingriffsbeurteilung zu machen, wurde bislang allerdings selten unternommen. Der Grund liegt in der Tatsache begründet, dass ökologische, geomorphologische und hydraulische Prozesse und die darauf aufbauenden Prozesse extrem komplex bzw. dynamisch sind. So ändern sich die Standortsbedingungen der Auenpflanzen, die meist an Störungsregime angepasst sind, ständig. Für die Erstellung solcher Modelle kann sicherlich das Projekt INFORM (Fuchs et al. 2003, Hettrich \& Rosenzweig 2002) als leitend gelten. Im gegenständlichen Projekt wird der Versuch unternommen im Falle der Vegetation ein conditional Vegetationsmodell im Gegensatz zu einem physiological - Vegetationsmodell zu erstellen (Zsuffa 2000).

Das Modell unseres Projektes orientiert sich am Duel's Rhine Matrix Modell - RMM (vergl. Zuffa, 2000) und besteht aus fünf Dimensionen:

- Statische Vegetationskarte auf Basis von Fernerkundungsdaten

- Geomorphologie, erstellt auf Basis eines digitalen Geländemodells

- Entfernung der einzelnen Landschaftselemente vom offen Wasser

- Flurabstand

- Bodenreife

Abgebildet werden die Daten in einer Matrix aus 5 x $5 \mathrm{~m}$ (Raster - Grid). Im Prinzip wird jede der dem Modell zugrundeliegende Dimension (Ausnahme statisches Vegetationsmodell) in Abhängigkeit vom jeweiligen Szenarium in das dynamisch zu bezeichnende Modell eingebracht. Aus dem Modell werden in der Folge die Ausdehnung der dynamischen $\mathrm{Au}$, der stabilen $\mathrm{Au}$, der Ufervegetation und der Pioniervegetationsanteile im Untersuchungsgebiet für jedes der Szenarien dokumentiert und als Indikatoren in die MCDA eingeführt.

Die Erstellung einer flächendeckenden Vegetationskarte

Da das Untersuchungsgebiet kaiserliches Jagdgebiet war und bis zum Beginn des 20. Jahrhunderts auch relativ unzugänglich war, sind Belege der historischen Vegetationsbedeckung nur im geringen Umfang vorhanden. Eigentlich erst nach dem Ende des Zweiten Weltkrieges rückte dieser Raum massiv in den Fokus vegetationskundlichen Arbeitens was zahlreiche Arbeiten (Margl 1973, Wendelberger 1975, Lazowski 1984, Grass 1993, Rotter 1999, Sauberer 1942 und Schratt-Ehrendorfer 2011) belegen. Diese Arbeiten und zahlreiche unveröffentlichte Erhebungen im Auftrag der Gemeinde Wien und der Nationalparkverwaltung liefern die Basis für die Erstellung eines statischen Vegetationsmodells, das weitgehend den Ist- Zustand der Landbedeckung im Untersuchungsgebiet abdeckt. Die meisten Daten liegen jedoch nur als Punktinformationen oder Transektinformationen vor. 
Eine flächendecken Vegetationskarte neueren Datums gibt es für das Untersuchungsgebiet nicht wird jedoch für das Vegetationsmodell benötigt.

Die gesamte Fläche des zu kartierenden Gebietes hat eine Ausdehnung von nahezu $33 \mathrm{~km}^{2}$. Flächendeckende Vegetationskarten können bei derartig großen Gebieten bei begrenzten zeitlichen und finanziellen Ressourcen nur durch die Erstellung einer hoch repräsentativen Stichprobe verbunden mit einer effizienten Freilandkampagne und einer darauf aufbauenden flächenhaften Umsetzung der Ergebnisse durch Verwendung raumanalytischer Informationssysteme (Fernerkundung und GIS) erstellt werden.

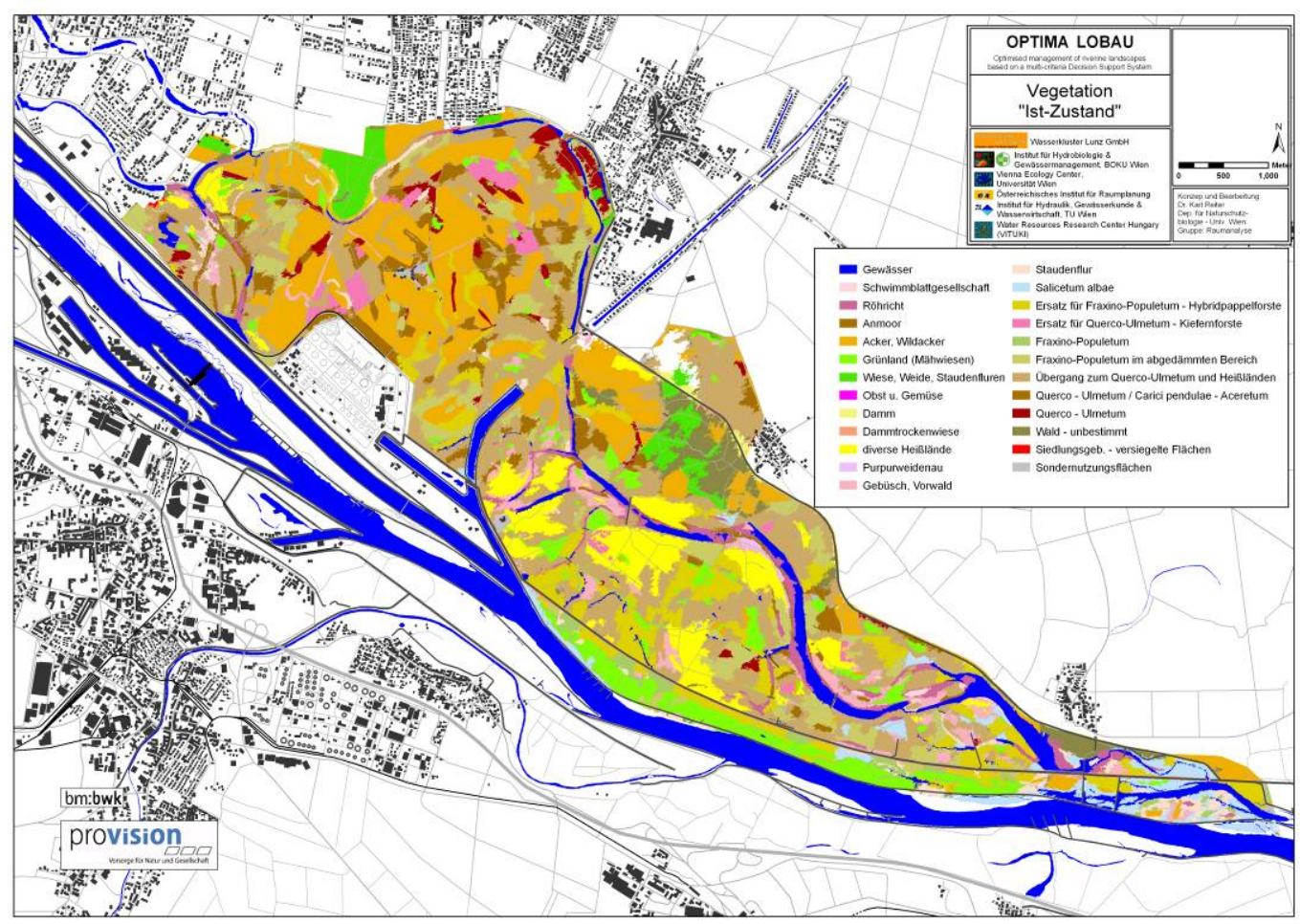

Abb. 70: Modellierte Vegetationskarte auf Basis der Bewertung der Forstkartierung der Gemeinde Wien und einer Bildsegmentierung

Die Vegetationsdaten (Vegetationsaufnahmen nach Braun-Blanquet 1962) der Stichprobe und vorhandene Daten zur Waldinventur (MA 49 - Forstamt der Gemeinde Wien) und eine visuelle Interpretation von CIR - Luftbildern durch die Firma Umweltdata im Auftrag der Österreichischen Bundesforste bzw. des Nationalparks Donauauen aus dem Jahre 1996 bilden für eine überwachte (supervised) Klassifikation des Luftbildes die Basis. Da für das Untersuchungsgebiet auch Daten direkt wirkender edaphischer, klimatischer, geomorphologisch-prozeßgebundener oder auch anthropogener Standortsfaktoren zum momentanten Zeitpunkt nicht vorhanden sind, müssen diese indirekt über Höhenmodelle erhoben werden. So können z.B. Bodenverhältnisse und folglich Auentypisierungen indirekt aus verschiedene Reliefformen bzw. Höhenstufen abgeleitet werden. 


\begin{tabular}{|l|l|}
\hline Ufergesellschaften & Forstlich genutzte Ersatzgesellschaften \\
\hline Schwimmblattgesellschaft & Ersatz für Querco-Ulmetum-Kiefernforste \\
\hline Anmoor & Ersatz für Fraxino-Populetum-Hybridpappelforste \\
\hline Röhricht & $\begin{array}{l}\text { Offenlandgesellschaften der dynamischen und } \\
\text { stabilen Au }\end{array}$ \\
\hline Purpurweidenau & Wiese, Weide, Staudenfluren \\
\hline Waldgesellschaften der dynamischen Au & Grünland (Mähwiesen) \\
\hline Salicetum albae & Damm \\
\hline Fraxino-Populetum & Dammtrockenwiese \\
\hline Fraxino-Populetum im abgedämmten Bereich & diverse Heißländen \\
\hline Gebüsch, Vorwald & Obst u. Gemüse \\
\hline Staudenflur & Acker, Wildacker \\
\hline Waldgesellschaften der stabilen Au & Siedlungsgeb. - versiegelte Flächen \\
\hline Querco-Ulmetum/Carici pendulae-Aceretum & Sondernutzungsflächen \\
\hline Übergang zum Qerco-Ulmetum und Heißländen & \multicolumn{2}{|l}{} \\
\cline { 1 - 1 } Querco-Ulmetum & \multicolumn{1}{|l|}{}
\end{tabular}

Tab. 24: Zusammenstellung der in der Vegetationskarte den IST-Zustand repräsentierenden Vegetationstypen (nach Reiter Endbericht OPTIMA Lobau) 


\begin{tabular}{|c|c|}
\hline Klasse Querco-Fagetea & Klasse Stellarietea mediae \\
\hline Ordnung Fagetalia sylvaticae & Ordnung Sisymbrietalia \\
\hline Verband Alnion incanae & Verband Malvion neglectae \\
\hline Unterverband Ulmenion & Hyoscyamo nigri-Malvetum neglectae \\
\hline Querco-Ulmetum & Klasse Artemisietea vulgaris \\
\hline Fraxino-Populetum, trocken & Ordnung Onopordetalia acanthii \\
\hline Fraxino-Populetum, untypisch & Verband Arction lappae \\
\hline Fraxino-Populetum, hart & Arctietum lappae \\
\hline Fraxino-Populetum, weich & Klasse Molinio- Arrhenatheretea \\
\hline Klasse Salicetea purpureae & Ordnung Arrhenatheretalia \\
\hline Ordnung Salicetalia purpureae & Verband Arrhenatherion \\
\hline Verband Salicion albae & Ranunculo bulbosi-Arrhenatheretum \\
\hline Salicetum albae & Ranunculo repentis-Alopecuretum pratensis \\
\hline $\begin{array}{l}\text { Salix purpurea- (Salicetea purpureae)- } \\
\text { Gesellschaft, untypisch }\end{array}$ & Pastinaco- Arrhenatheretum \\
\hline Klasse Phragmiti-Magnocaricetea & Tanaceto- Arrhenatheretum \\
\hline Ordnung Phragmitetalia & Verband Cynosurion \\
\hline Verband Phragmition communis & Lolio-Cynosuretum \\
\hline Phragmitetum vulgaris & Ordnung Potentillo- Polygonetalia \\
\hline Verband Magnocaricion elatae & Verband Potentillion anserinae \\
\hline Unterverband Caricenion rostratae & Dactylido- Festucetum arundinaceae \\
\hline Caricetum elatae & Rumici crispi- Agrostietum stoloniferae \\
\hline Caricetum acutiformis & Klasse Festuco- Brometea \\
\hline Verband Caricenion gracilis & Ordnung Festucetalia valesiacae \\
\hline Phalaridetum arundinaceae & Verband Festucion valesiacae \\
\hline Ordnung Nasturtio- Glycerietalia Pignatti & Teucrio botryos- Andropogonetum \\
\hline Verband Phalaridion arundinaceae & Ordnung Brometalia erecti \\
\hline Rorippo-Phalaridetum & Verband Bromion erecti \\
\hline Klasse Galio- Urticetea & Onobrychido- Brometum \\
\hline Ordnung Convolvuletalia sepium & Verband Cirsio- Brachypodion pinnati \\
\hline Verband Senecionion fluviatilis & Klasse Rhamno- Prunetea \\
\hline Convolvulo-Archangelicetum & Ordnung Prunetalia \\
\hline $\begin{array}{l}\text { Andere Gesellschaften des Verbandes } \\
\text { Senecionion fluviatilis }\end{array}$ & Verband Berberidion \\
\hline Solidago canadensis- Gesellschaft & Ligustro- Prunetum \\
\hline
\end{tabular}

Tab. 25: Liste der auf Basis der Vegetationserhebung (Mayer 2007) ausgewiesenen Syntaxon

\section{$\underline{\text { Karte zur potentiellen Waldvegetation }}$}

Um die errechneten Daten bzw. Grenzen der Vegetation verifizieren zu können, wurden im Freiland des gesamten Donauraums östlich von Wien - inklusive Lobau - 150 Einzelpunkte in verschiedenen Höhenlagen sowie zehn Querprofile mit insgesamt über 1200 Punkten vegetationsökologisch kartiert d.h. die Punkte wurden mit Vegetationsaufnahmen bzw. Artenlisten dokumentiert. Alle Freilanderhebungen im Untersuchungsgebiet wurden in Bezug zu analysierten Daten aus dem hochauflösenden Höhenmodell gesetzt. Für die einzelnen Baumarten wurde ein sogenannter „ecological envelop“ bezogen auf die Geländehöhe bestimmt, d.h. es wurde die Höhenamplitude in cm über Mittelwasser der einzelnen Baumarten bestimmt). Aus dem Wissen um die Verteilung der einzelnen Baumarten in der Höhenerstreckung im Aubereich östlich von Wien konnte auch für die einzelnen Vegetationstypen, die die Bäume jeweils aufbauen, eine Höhenamplitude erstellt werden (Tab. 26). Durch eine vegetationskundliche bzw. synsystematische Analyse der gemachten Vegetationsaufnahmen wurden Vegetationstypen bestimmt. So konnte neben der Erstellung des „ecological envelop“ für jede Baumart indirekt (über die einzelnen Arten der Vegetationsaufnahme) und direkt (Verschneidung des Mittelpunktes der Aufnahmefläche mit dem Höhenmodell) auch der 
„ecological envelop“ für Vegetationstypen in ihrer Gesamtheit bestimmt werden. Da nur die Geländehöhe als Faktor für die Parametrisierung des Modells unter dem Gesichtspunkt der hohen Komplexität der Auenvegetation zu wenig ist, wurde auch noch die Karte der dynamischen bzw. stabilen $\mathrm{Au}$ (Abb. 71) für die Parametriesierung des Modells herangezogen.

\begin{tabular}{|l|l|}
\hline \multicolumn{2}{|l|}{ Stabile Au } \\
\hline 80 & 209: Weidenau der stabilen $\mathrm{Au}$ \\
\hline 210 & 279: feuchte Feldulmen - Eschenau $\mathrm{Au}$ \\
\hline 280 & 379: Feldulmen - Eichenau \\
\hline 380 & 430: Eichen - Lindenau \\
\hline \multicolumn{2}{|l|}{ Dynamische Au } \\
\hline 80 & 159: Weidenau \\
\hline 160 & 289: feuchte Pappelau \\
\hline 290 & 379: frische Pappelau \\
\hline 380 & 470: Schwarzpappelau \\
\hline
\end{tabular}

Tab. 26: Höhenabstufung in cm der einzelnen erarbeiteten Vegetationstypen

\section{Potentiell Natürliche Vegetation}

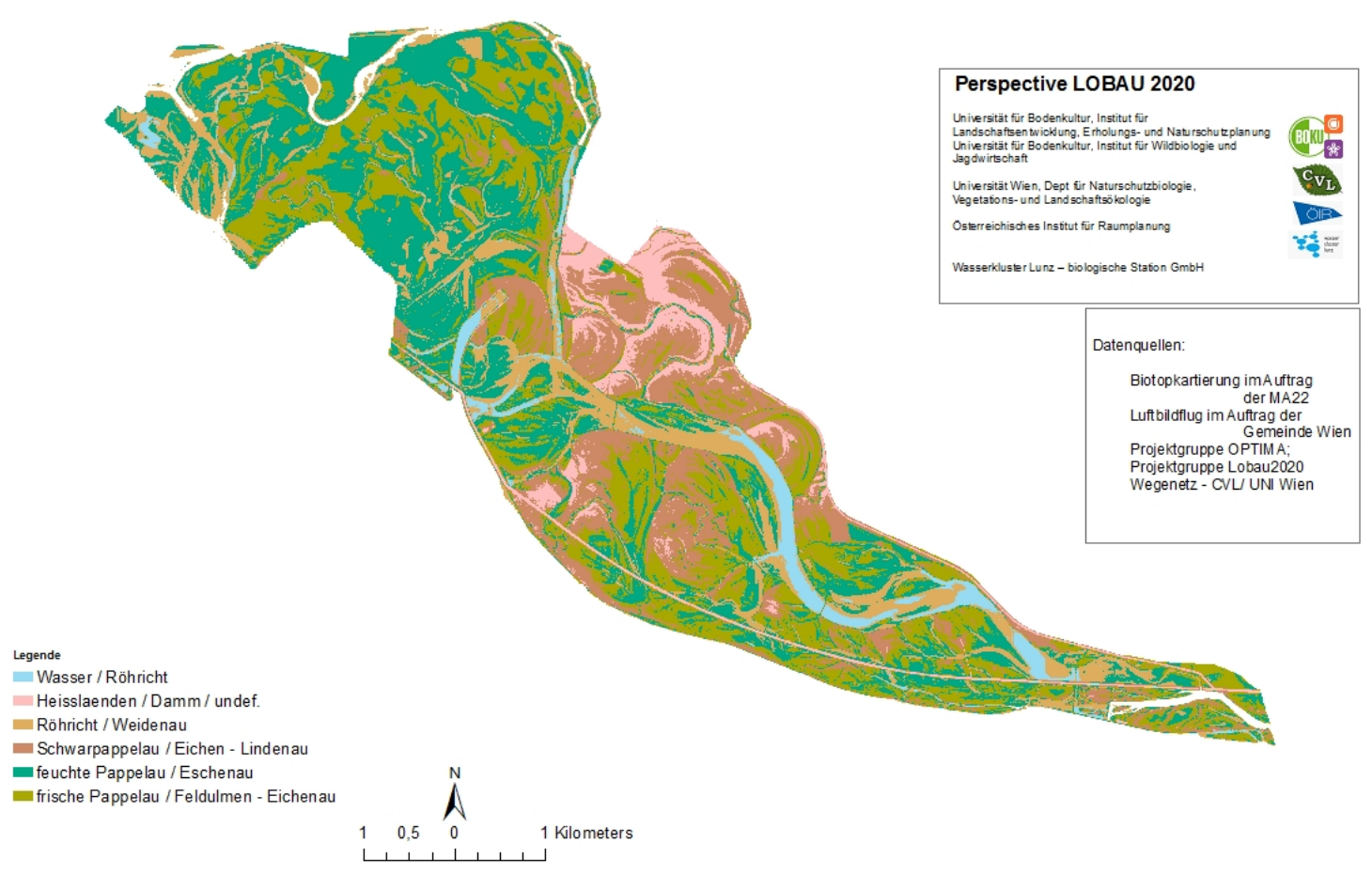

Abb. 71: Karte der Potentiell Natürlichen Vegetation im Gebiet der Lobau

Aus den Werten ist erkennbar, dass es sehr oft eine Überschneidung der Zonengrenzen gibt. Der Umstand, dass sich aber in den Bereichen der dynamischen und der stabilen Au unterschiedliche Auwaldgesellschaften auf derselben relativen Höhe befinden, ist hauptsächlich auf die Bodenreife und den Bodenaufbau zurückzuführen. Diese Parameter bezogen auf Auentyp (dynamisch, stabil) und Geländehöhe kamen nur für die Waldflächen zur Anwendung. Alle Nicht -Waldflächen wurden aus der Luftbildinterpretation zu den Biotoptypen der Firma Umweltdata übernommen. So entstand aus der Karte der interpretierten Biotoptypen und der modellierten Karte der Waldtypen eine Karte, die flächendeckend die Vegetation im Untersuchungsgebiet widerspiegelt. Diese Karte muss eigentlich als Karte der PNV (potentiell natürliche Vegetation) aufgefasst werden und bildet eine ideale Grundlage für die Modellansätze wie zuvor vorgestellt. 
$\underline{\text { Karte zur Hemerobie der Wälder der Lobau }}$

Auswahl der Punkte

Vorgabe war die Auswahl von Waldinventurpunkten, die nach der Wiener Biotopkartierung waldtragend sind und von Wegen maximal 50 Meter entfernt sind. Die konkrete Vorgehensweise war nun die Erstellung eines GIS -Layers der Flächen mit der Vorgabe „,waldtragend“ und in einem Buffer von 50 Metern neben den Wegen liegend. Aus diesem Datensatz wurden dann durch Zufallswahlverfahren drei Punkte der Waldinventur pro Waldtyp für die Erhebung gewählt. Diese Punkte stehen somit repräsentativ für die Waldflächen des Untersuchungsgebietes und bilden die Basis einer Interpolation von Punkterhebungen in die Fläche (Abb. 72).

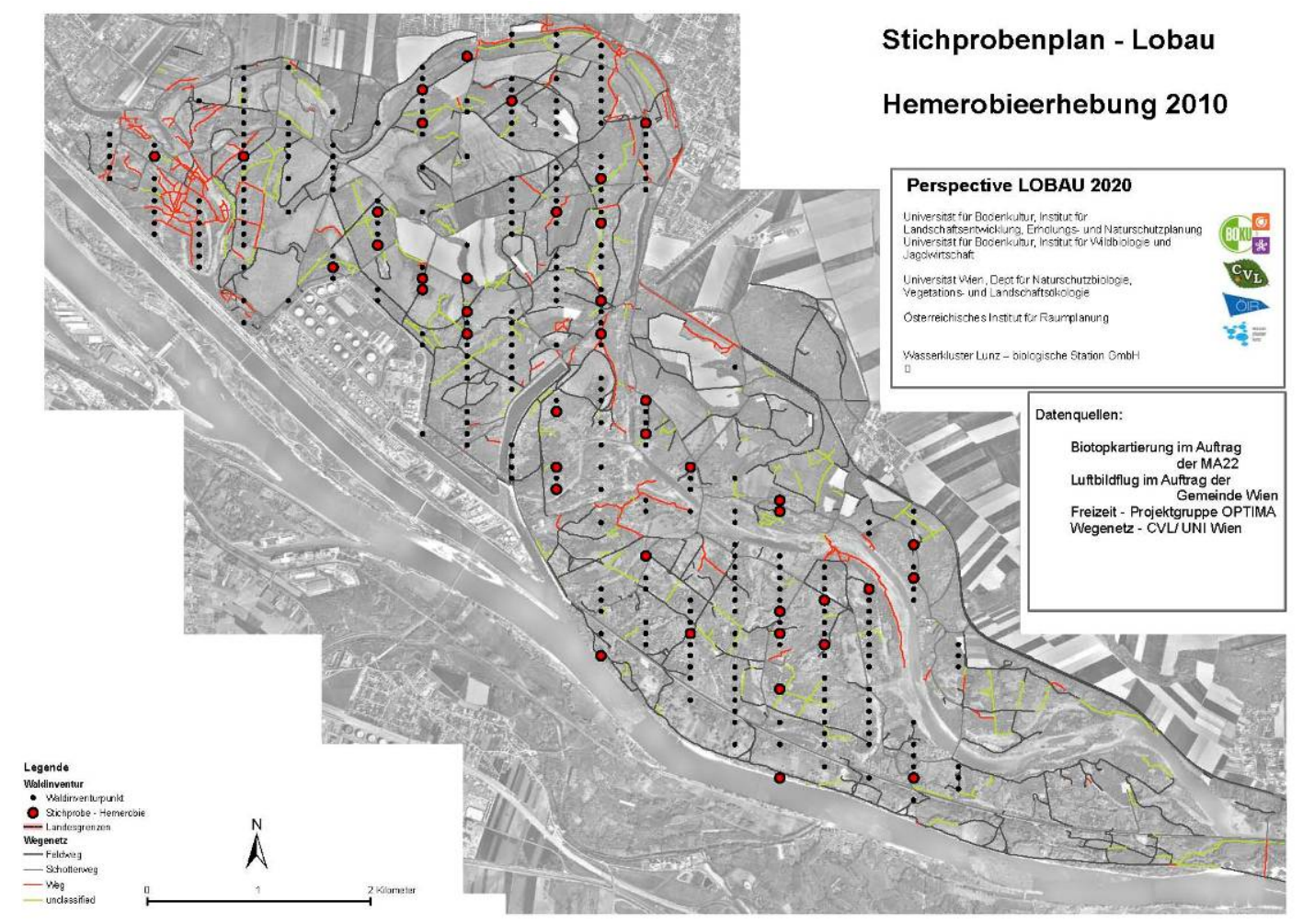

Abb. 72: Karte der Verteilung der Probepunkte zur Untersuchung der Hemerobie

Hemerobieberechnung

Zur Beschreibung der Naturnähe der Wälder wird das Hemerobiekonzept (Jalas 1955; Sukopp 1972; Kowarik, 1988 u. a.) in Kombination mit einer modifizierten Ökologischen Wertanalyse nach Ammer \& Utschick (1984) angewendet. Folgende Hemerobie- und Bestandeskriterien wurden aufgenommen: Baumartenanteile, Brusthöhendurchmesser der fünf stärksten Bäume, Entwicklungsphasenansprache oder Wuchsklassenerfassung, die Beurteilung von Beeinträchtigungen und Nutzungen, wie touristische Nutzung, Forstliche Endnutzung- und Vornutzung, Wildeinfluss etc. Die Verjüngung wurde ebenso wie das Totholz erfasst. Diese erhobenen Daten werden im Rahmen der Hemerobiebewertung elf Kriterien zugewiesen.

Danach werden diese Einzelkriterien dichotom verknüpft und dabei, je nach Beeinflussung des Hemerobiegrades, gewichtet. Zur Verknüpfung werden folgende unterschiedliche Methoden angewendet: arithmetisches Mittel, Zuschlagverfahren oder logische Kombination (Berechnung aus vorgegebenen Matrizen). Es entsteht ein Dendrogramm mit dem Hemerobiewert als Endergebnis (Abb. 73). Die Berechnung enthält somit Einzelkriterien und aggregierte Kriterien, für die jeweils sogenannte Relativwerte (Bewertungen von 1 bis 9) berechnet wurden, damit Transparenz, Nachvollziehbarkeit und eine differenzierte Interpretation der Hemerobiewerte gegeben ist (Tab. 27). (Detailinformation siehe Illedits \& Zsak, 2012). 


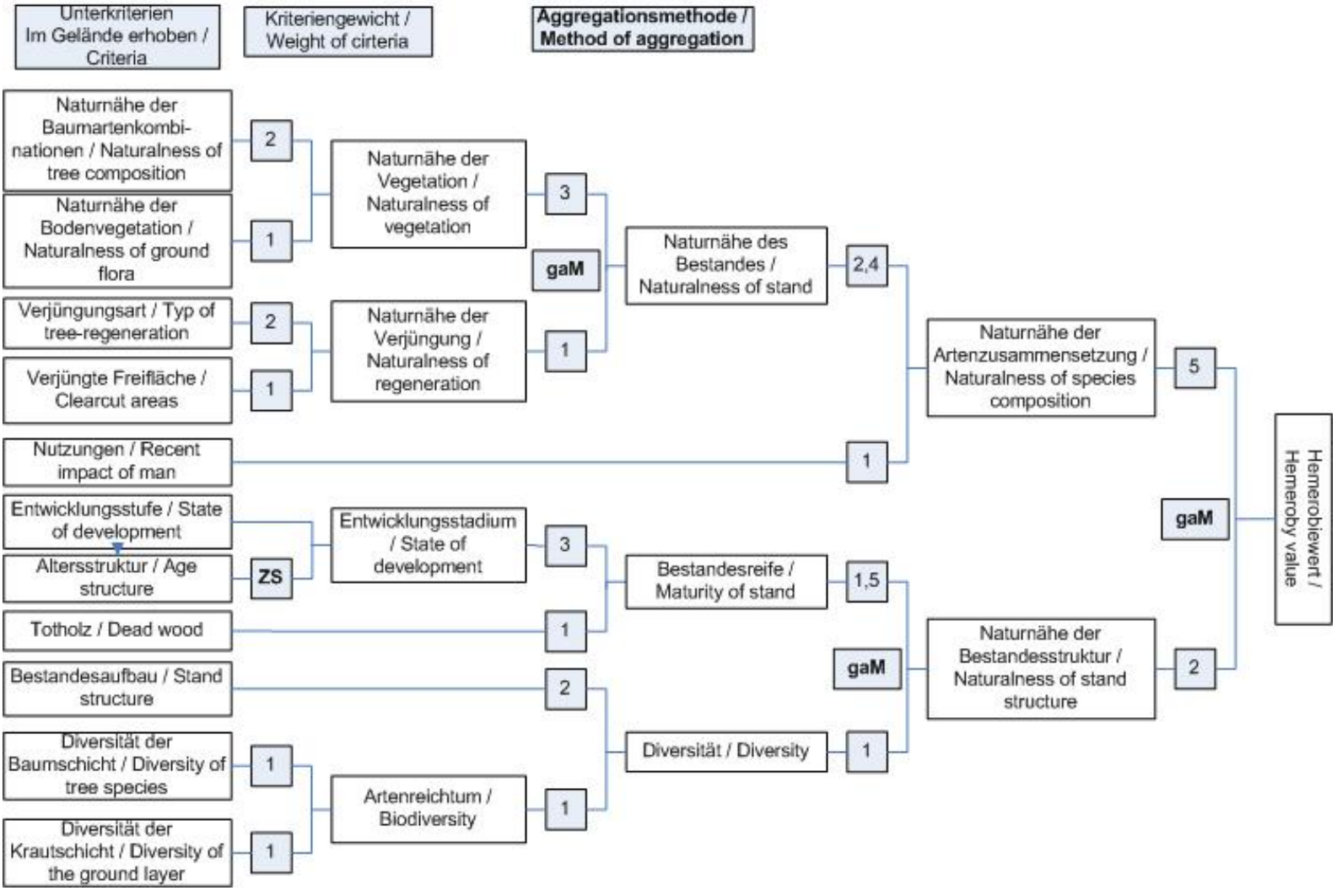

Abb. 73: Verknüpfungsbaum der Hemerobiebewertung (aus Enzenhofer et al., 2009)

\begin{tabular}{|c|l|l|}
\hline \multicolumn{2}{|c|}{ Hemerobiegrade } & \multirow{2}{*}{ Naturnäheklassen } \\
\hline 1 & polyhemerob & \multirow{2}{*}{ künstlich } \\
\hline 2 & $\alpha$-euhemerob & \\
\hline 3 & $\beta$-euhemerob & \multirow{2}{*}{ stark verändert } \\
\hline 4 & $\alpha$-mesohemerob & \\
\hline 5 & $\beta$-mesohemerob & \multirow{2}{*}{ mäßig verändert } \\
\hline 6 & $\alpha$-oligohemerob & \\
\hline 7 & $\beta$-oligohemerob & \multirow{2}{*}{ naturnah } \\
\hline 8 & $\gamma$-oligohemerob & \\
\hline 9 & ahemerob & natürlich \\
\hline
\end{tabular}

Tab. 27: Neunstufige Hemerobieskala und die fünf Stufen der Naturnähe (Grabherr et al., 1998)

Interpolation der Punktinformation

Die Stichprobe bestehend aus ca. 40 Probepunkten bildet die Basis für die Interpolation der Punktinformation (Hemerobiewert) in die Fläche mit Hilfe der Methodeninventare des Programms ArcGIS-Desktop. Für alle Waldvegetationstypen, welche jeweils durch mehrere Felderhebungen dokumentiert sind, wurde durch Frequenzanalysen der charakteristische Hemerobiewert errechnet. Dieser so gewonnene Wert wurde dann allen Flächenanteilen des jeweiligen Waldvegetationstyps zugewiesen (Tab. 28, Abb. 74, Abb. 75). 


\begin{tabular}{|c|c|c|c|}
\hline Waldbiotoptyp & $\begin{array}{c}\text { Hemerobiewert } \\
\text { der Einzelflächen }\end{array}$ & Median & Hemerobiestufe \\
\hline \multirow{3}{*}{ Ahornforst } & 4 & 4 & alpha-mesohemerob \\
\hline & 4 & & \\
\hline & 5 & & \\
\hline \multirow{2}{*}{ Junge Laubbaumaufforstung } & 4 & 5,5 & beta-mesohemerob/alpha \\
\hline & 7 & & \\
\hline \multirow{2}{*}{ Robinienforst } & 4 & 5 & beta-mesohemerob \\
\hline & 6 & & \\
\hline \multirow{3}{*}{ Schwarzpappelauwald } & 4 & 6 & alpha-oligohemerob \\
\hline & 6 & & \\
\hline & 6 & & \\
\hline Silberpappel- und Weidenforst & 4 & 4 & alpha-mesohemerob \\
\hline \multirow{11}{*}{ Silberpappelauwald } & 4 & 6 & alpha-oligohemerob \\
\hline & 6 & & \\
\hline & 6 & & \\
\hline & 6 & & \\
\hline & 6 & & \\
\hline & 6 & & \\
\hline & 7 & & \\
\hline & 7 & & \\
\hline & 7 & & \\
\hline & 7 & & \\
\hline & 8 & & \\
\hline Weidenauwald & 4 & 4 & alpha-mesohemerob \\
\hline \multirow{8}{*}{ Eiche-Ulmen-Eschen-Auwald } & 6 & 6 & alpha-oligohemerob \\
\hline & 6 & & \\
\hline & 6 & & \\
\hline & 6 & & \\
\hline & 6 & & \\
\hline & 6 & & \\
\hline & 7 & & \\
\hline & 7 & & \\
\hline Eschenforst & 6 & 6 & alpha-oligohemerob \\
\hline \multirow{2}{*}{ Hybridpappelforst } & 5 & 5,5 & beta-mesohemerob/alpha \\
\hline & 6 & & \\
\hline \multirow{2}{*}{$\begin{array}{l}\text { Laubbaummischforst aus } \\
\text { einheimischen Baumarten }\end{array}$} & 5 & 5,5 & beta-mesohemerob/alpha \\
\hline & 6 & & \\
\hline Nadelbaummischforst aus & 5 & 5 & beta-mesohemerob \\
\hline Rotföhrenforst & 5 & 5 & beta-mesohemerob \\
\hline Schwarzföhrenforst & 5 & 5 & beta-mesohemerob \\
\hline
\end{tabular}

Tab. 28: Hemerobiestufen der Stichprobenklassen bzW. der Waldbiotoptypen (aus Illedits \& Zsak, 2012) 
HEMEROBIEERHEBUNG - LOBAU 2011

\section{Hemerobiestufen der Waldökosysteme}
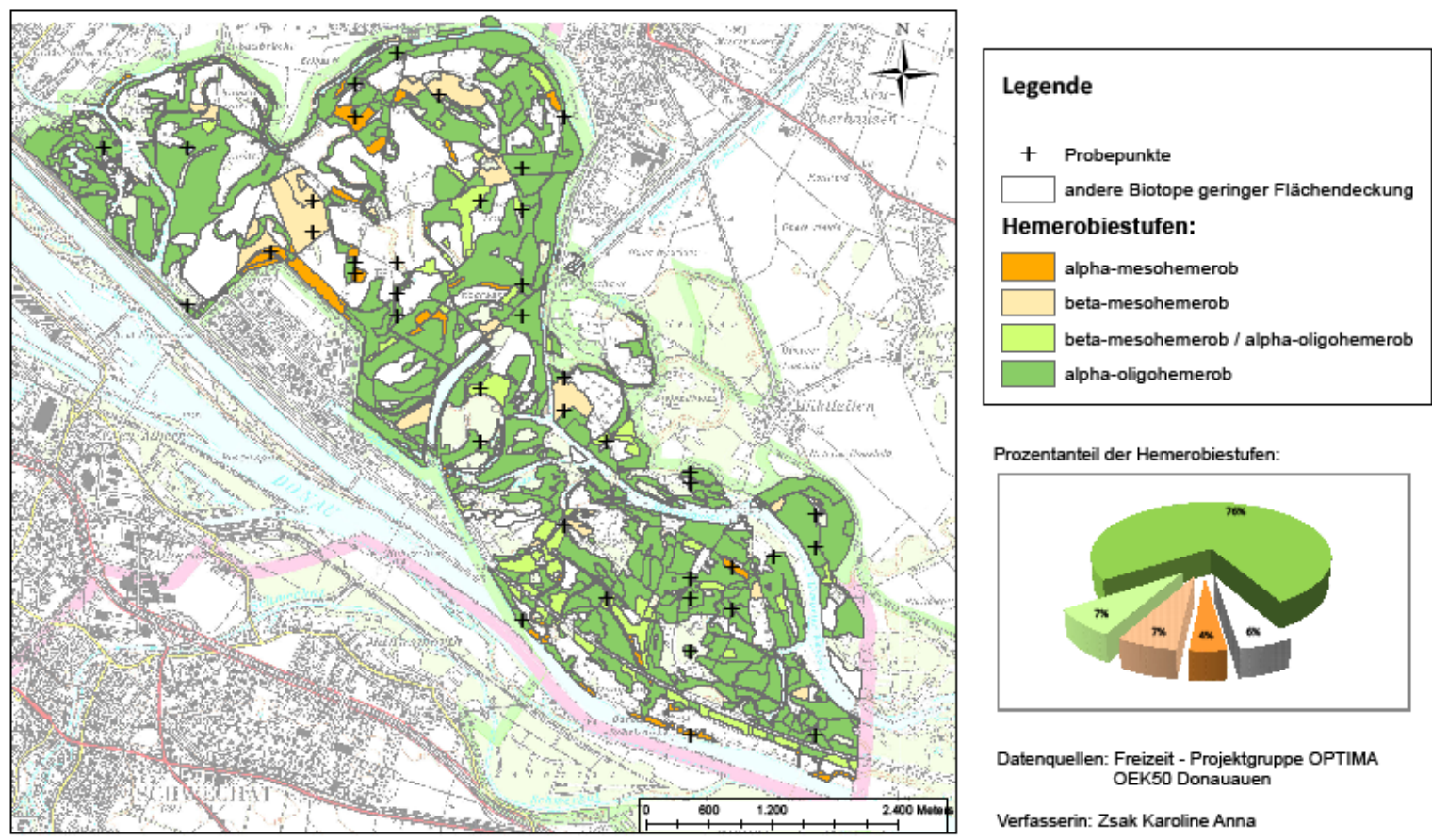

Prozentanteil der Hemerobiestufen:

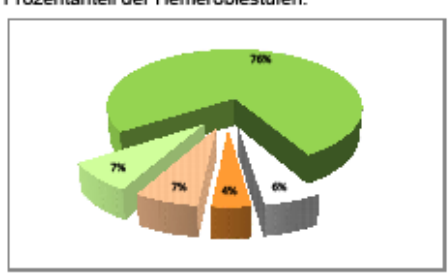

Datenquellen: Freizeit - Projektgruppe OPTIMA OEK50 Donauauen

Verfasserin: Zsak Karoline Anna

Abb. 74: Karte der Hemerobie der Waldökosysteme der Lobau - (aus Illedits \& Zsak, 2012)

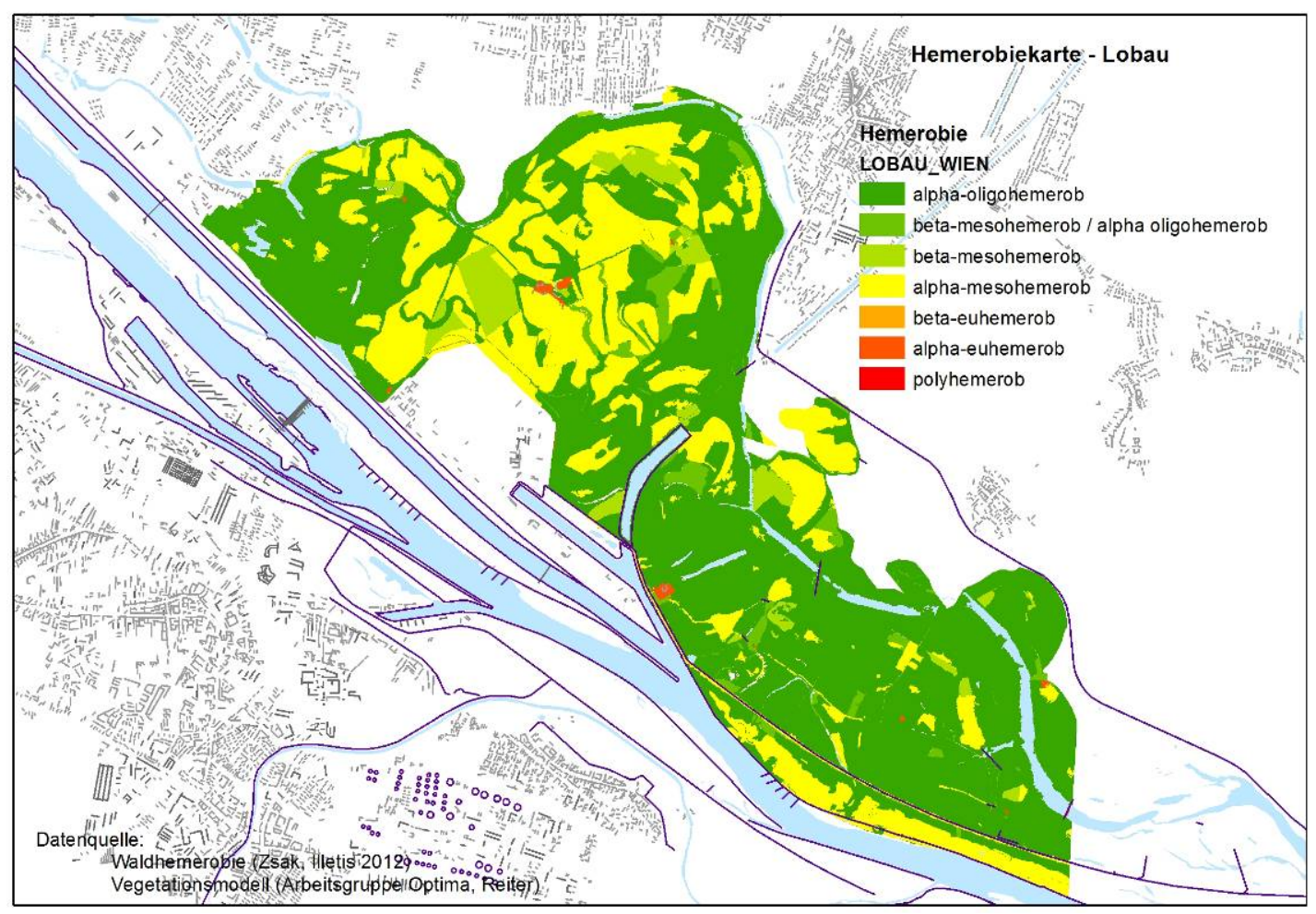

Abb. 75: Karte der Hemerobie der Lobau - kompiliert aus der Karte der Hemerobie - Waldökosystem und der gutachterlichen Zuweisung von Hemerobiewerte bei Offenland Vegetationstypen und Vorwaldstadien. 
Sensibilitätskarte Vegetation Lobau

Die Sensibilitätskarte leitet sich aus der Hemerobiekarte (Abb. 75) folgend den Zuweisungen aus Tab. 28 ab. Gegenübergestellt wird die Ist- Situation Abb. 76) und die Verteilung der Sensibilität der Vegetation bei einem Gewässer-Szenario ( $1 \mathrm{~b}$ aus dem Projekt Optima-Lobau, Abb. 77).

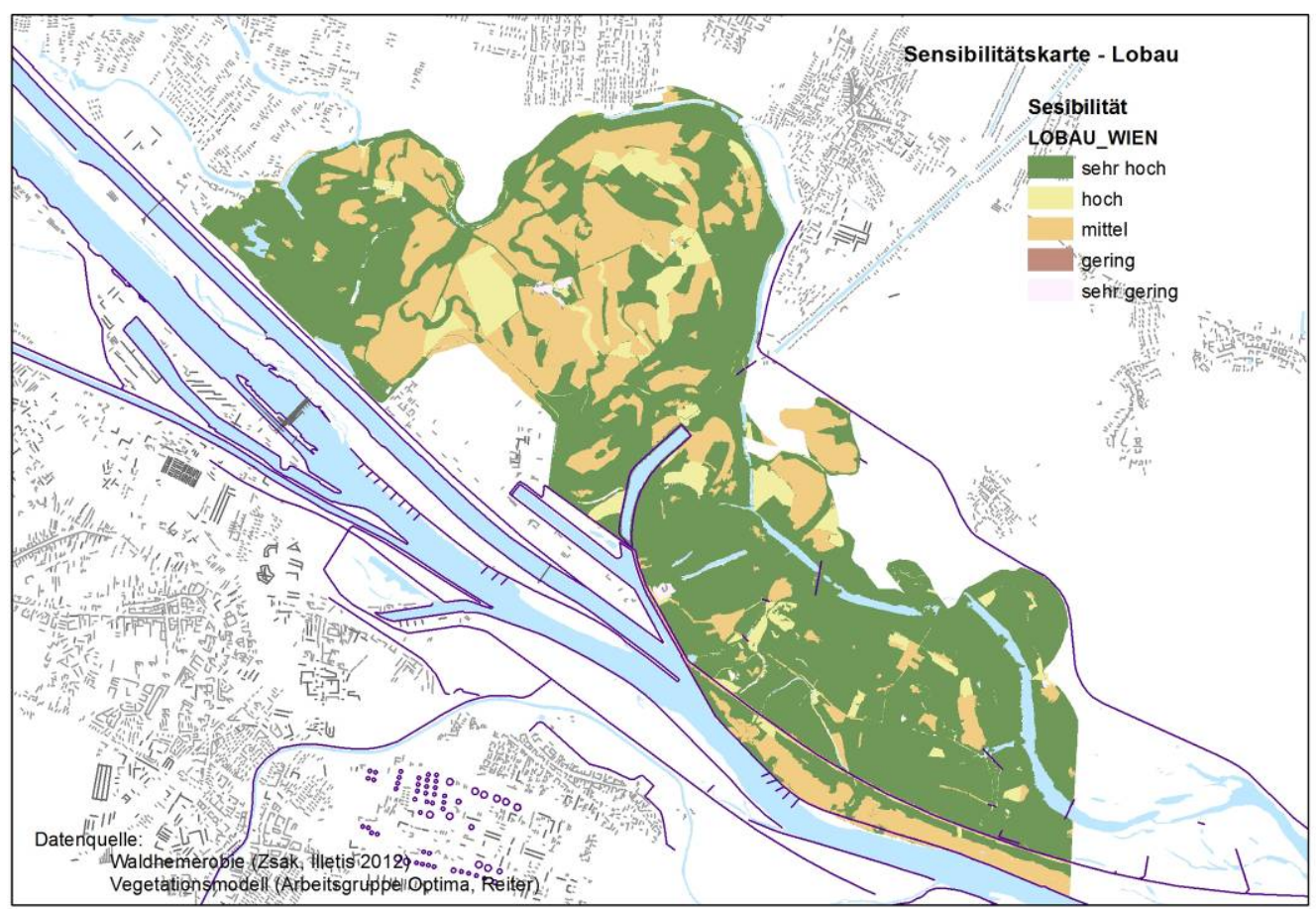

Abb. 76: Karte der Sensibilität der Ökosysteme in der Lobau - abgeleitet aus der Karte zur Hemerobie.

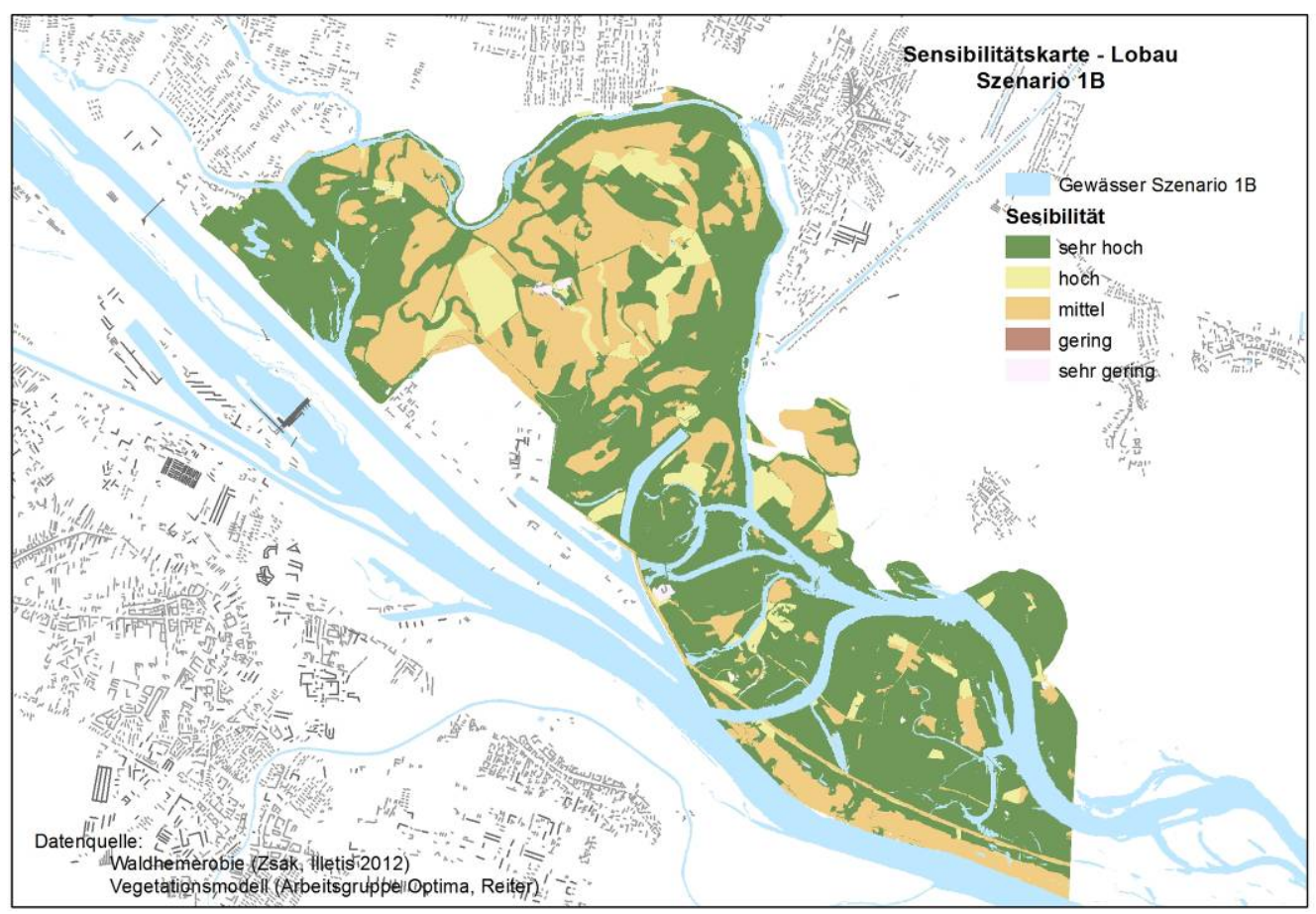

Abb. 77: Karte der Sensibilität der Ökosysteme in der Lobau - abgeleitet aus der Karte zur Hemerobie. 


\begin{tabular}{|l|l|}
\hline \multicolumn{1}{|c|}{ Hemerobiestufen } & \multicolumn{1}{c|}{ Sensibilität } \\
\hline alpha-euhemerob & sehr gering \\
\hline beta-euhemerob & gering \\
\hline alpha-mesohemerob & mittel \\
\hline beta-mesohemerob & hoch \\
\hline beta-mesohemerob / alpha-oligohemerob & hoch \\
\hline alpha-oligohemerob & sehr hoch \\
\hline
\end{tabular}

Tab. 29: Zuweisungstabelle Hemerobiestufen - Sensibilität

Bewertung der Besucherfrequenzen

Für die Darstellung der Auswirkungen von unterschiedlichen Besucherfrequenzen auf die Sensibilität der Vegetationsdecke des Untersuchungsgebietes wurden nur vier Szenarien gewählt:

- Frequenz 1999 Besucheranzahl - Werktag / Sommer (Szenario Nr.: 02)

- Frequenz 2025 Besucheranzahl - Werktag / Sommer (Senario Nr.: 04)

- Frequenz 2025 Besucheranzahl - Werktag - Szenario 1b / Sommer (Senzario Nr.: 06)

- Frequenz 2025 Besucheranzahl - Werktag - Szenario 1b / Sommer; mit Maßnahmen im Lobau - Umfeld, welche in die Fläche gehen (Szenarion Nr.: 14)

Aus den Besucherfrequenzen wurden für die jeweiligen Wegabschnitte sogenannte "Besucher - HotSpots" errechnet und diese dann in Beziehung zur Sensibilität der begleitenden Vegetation gestellt. Der Analyse zu Grunde liegende Hypothese lautet: hoher Besucherdruck wirkt sich auf sensible Bereiche negativ aus.

Für die Klassifizierung der Wegabschnitte bezogen auf ihre modellierte Besucherfrequenz kam das Verfahren der Hot Spot Analysis (Getis-Ord Gi*) zur Anwendung. Basierend auf einem gegebenen Set von gewichteten Elementen (hier die Besucherfrequenz pro Wegabschnitt) werden Cluster mit hoher Besucherfrequenz („Hot Spots“) und solche mit geringer Besucherfrequenz („Cold Spots“) errechnet. Die Karten der Hot- bzw. Cold- Spots der Wegabschnitte werden in Beziehung zu den beiden Sensibilitätskarten (Abb. 78) gesetzt und durch einfache Wegstreckensummenanalysen dokumentiert. 


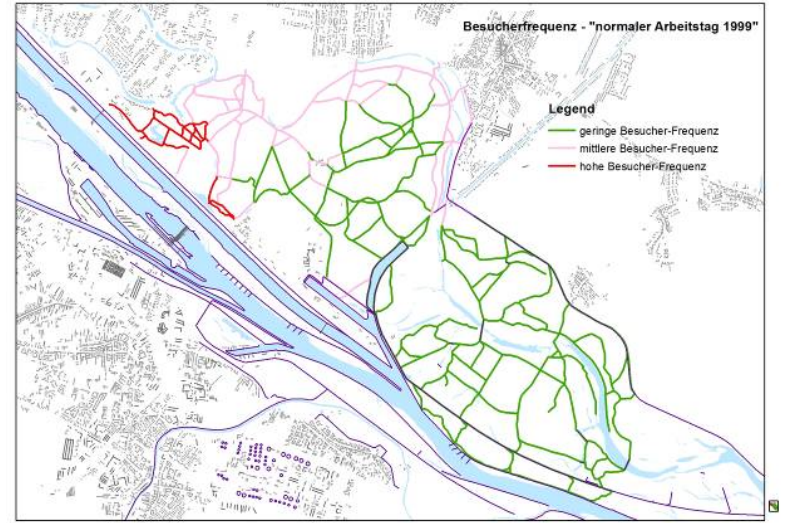

a.

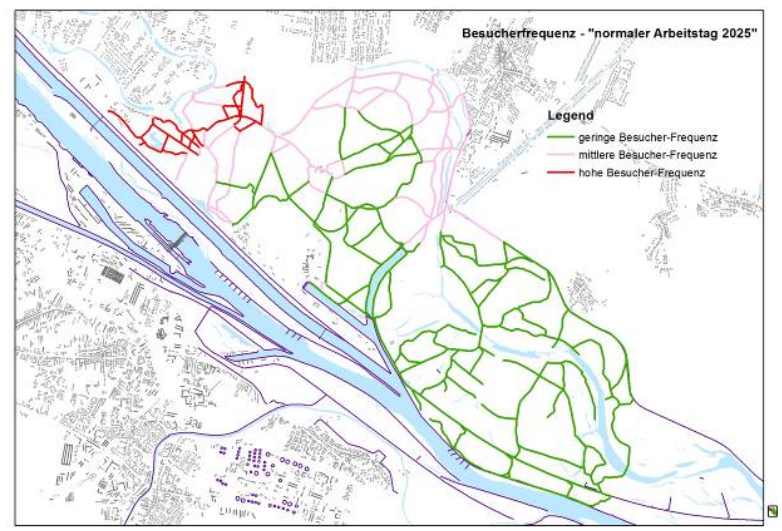

c.

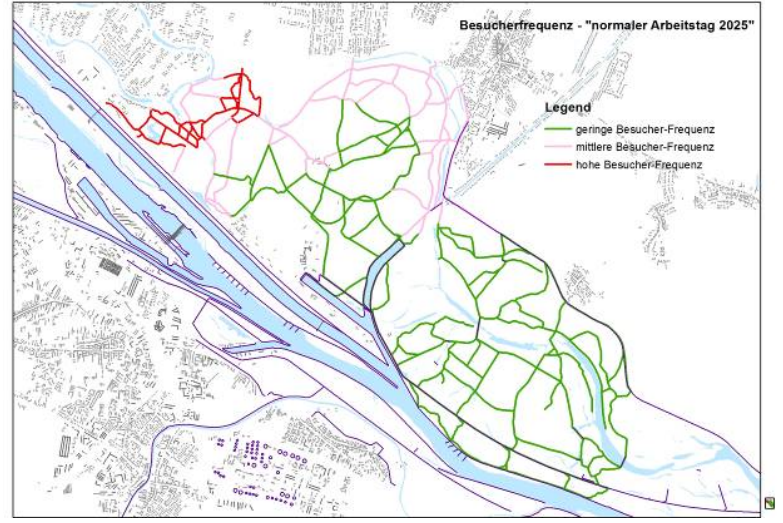

b.

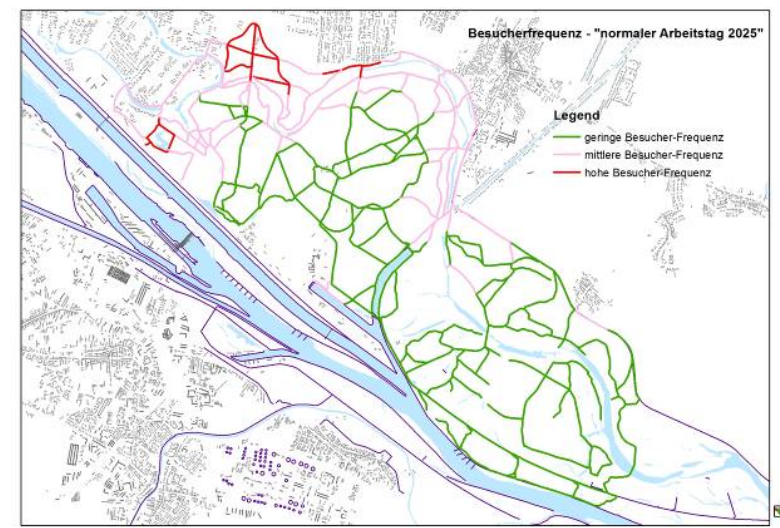

d.

Abb. 78: Karten der klassifizierten Besucherfrequenz an einem normalen Arbeitstag durch Hot Spot Analysis der Besucherfrequenz und einer Verteilung auf drei Klassen durch Equal Interval; Karte a: Normaler Arbeitstag 1999; Karte b: Normaler Arbeitstag 2012; Karte c: Normaler Arbeitstag unter Einbeziehung des Gewässerszenarios 1b; Karte d: Normaler Arbeitstag unter Einbeziehung des Gewässerszenarios $1 b$ und einer flächigen Ausprägung der Vorlandszenarien

\subsubsection{Ergebnisse und Diskussion (Illedits \& Zsak, 2012)}

Bei einem Vergleich mit den Resultaten der MaB-Hemerobiestudie für die österreichischen Waldökosysteme fiel auf, dass die Beschreibung der Nutzungsintensität und Bewirtschaftung, sowie die allgemeine Darstellung der Wälder der jeweiligen Hemerobiestufen mehr oder weniger stark vom Bild der Waldökosysteme im Untersuchungsgebiet der Lobau, welche nach Berechnung dem gleichen Hemerobiegrad entsprechen würden, abweichen. So wird zum Beispiel für die „naturnahen Waldflächen,, auf ein Fehlen natürlicher Zerfallsphasen mit charakteristischem Totholzvorkommen hingewiesen (vgl. Grabherr et al., 1998).

Diese Beschreibung trifft auf die im Zuge der Hemerobieerhebung als „beta-oligohemeroben“ bzw. „gamma-oligohemeroben“ beurteilten Waldflächen nicht zu, was durch die überwiegend hohen Relativwerte des Kriteriums „Totholz“ auf diesen Flächen bestätigt werden kann. Weiters vermittelt die Darstellung der „alpha-oligohemeroben“ und „beta-mesohemeroben“ Wälder in Grabherr et al. (1998) als „durchwegs forstwirtschaftlich intentsiv genutzt“ (zitiert aus Grabherr et al., 1998) ein negativeres Bild, als dies auf die Erhebungsflächen derselben Hemerobiestufen in der Wiener Lobau tatsächlich zutrifft, welche keiner bzw. lediglich historisch einer forstlichen Endnutzung unterstanden hat. 


\begin{tabular}{|l|c|c|}
\hline \multicolumn{1}{|c|}{ Hemerobiestufe } & $\begin{array}{c}\text { Gesamtfläche im } \\
\left.\text { Untersuchungsgebiet } \mathbf{( m}^{\mathbf{2}}\right)\end{array}$ & $\begin{array}{c}\text { Prozentanteil an der } \\
\text { Gesamtfläche (\%) }\end{array}$ \\
\hline ohne Angabe & 762643,9551 & 5,96 \\
\hline alpha-mesohemerob & 520814,2767 & 4,06 \\
\hline beta-mesohemerob & 841081,5478 & 6,57 \\
\hline $\begin{array}{l}\text { beta-mesohemerob/alpha- } \\
\text { oligohemerob }\end{array}$ & 964015,4745 & 7,53 \\
\hline alpha-oligohemerob & 9716858,471 & 75,88 \\
\hline
\end{tabular}

Tab. 30: Flächenanteile der Hemerobiestufen im Untersuchungsgebiet (aus Illedits \& Zsak, 2012)

Eine Begründung für die unterschiedlichen Merkmalsausprägungen der Hemerobiestufen findet sich in der Kombination unterschiedlicher Faktoren, welche nach Aggregation ein und dieselbe Beurteilung der Hemerobie ergeben können. Daher ist die Beschreibung eines charakteristischen, „typischen“ Erscheinungsbildes für eine bestimmte Hemerobiestufe unrealistisch. (vgl. Grabherr et al. 1998). Glavac (1996) weist darauf hin, dass eindeutige Unterscheidungsmerkmale, für die Einstufung der Hemerobie für ein bestimmtes Gebiet, fehlen und diese daher nicht werturteilsfrei sein kann. Glavac (1996) beschreibt weiter die oligohemerobe Stufe nach Sukopp et al. (1974), Blume \& Sukopp (1976) und Bornkamm (1980) als Vegetationseinheiten, welche unter geringem Einwirken des Menschen wenig Veränderung zeigen, so liegt der zu erwartende Neophyten- und Therophytenanteil bei $<5 \%$ bzw. $<20 \%$. Als Beispiel führt er unter anderem forstlich ungenutzte Weich- und Hartholzauen an. Mesohemerobe Flächen wären danach z.B Forstflächen mit einer standortsfremden Baumartenkombination mit einem Neophytenanteil von $5-12 \%$ und einem Therophytenanteil von < $20 \%$. (vgl. Glavac 1996).

Diese allgemeine Darstellung der Hemerobieerhebung für die Waldflächen der Lobau resultierenden Hemerobiestufen, widerspiegelt die allgemeine Beschreibung des Untersuchungsgebietes relativ gut (aus Illedits \& Zsak, 2012). Dementsprechend gilt die Hemerobie quasi als Proxy der ökologischen Situation eines Gebietes.

Besucherdruck als Vektor, der in Richtung einer Änderung der Hemerobie hin zu einem größeren Kultureinfluss weist, zeigt ganz klar welche Bereiche als sensibel bzw. weniger sensibel aufzufassen sind. Sofern es um die Hemerobie aus ökologischer Sicht in einem bestimmten Landschaftsausschnitt nicht besonders gut bestellt ist, kann der zu erwartende Besucherdruck weniger Schaden anrichten als in jenen Bereichen, wo Kultureinfluss gering und dementsprechend die Sensibilität hoch ist.

Die Grafiken der Abb. 79 zeigen, dass der Großteil der Wege eher wenig genutzt wird aber hier dann doch zum größeren Teil sensible Bereiche tangiert. In allen untersuchten Szenarien zeigt sich, dass die Bereiche mit sehr hoher Sensibilität auch hohem Besucherdruck ausgesetzt sind - wobei aber gerade das Beispiel mit zukünftigen Maßnahmen in der Fläche des Vorlandes deutlich zeigt, dass die bessere Verteilung der BesucherInnen bzw. auch die Reduktion von Wegen durch neue Gewässerarme die sensiblen Bereiche entlastet. 

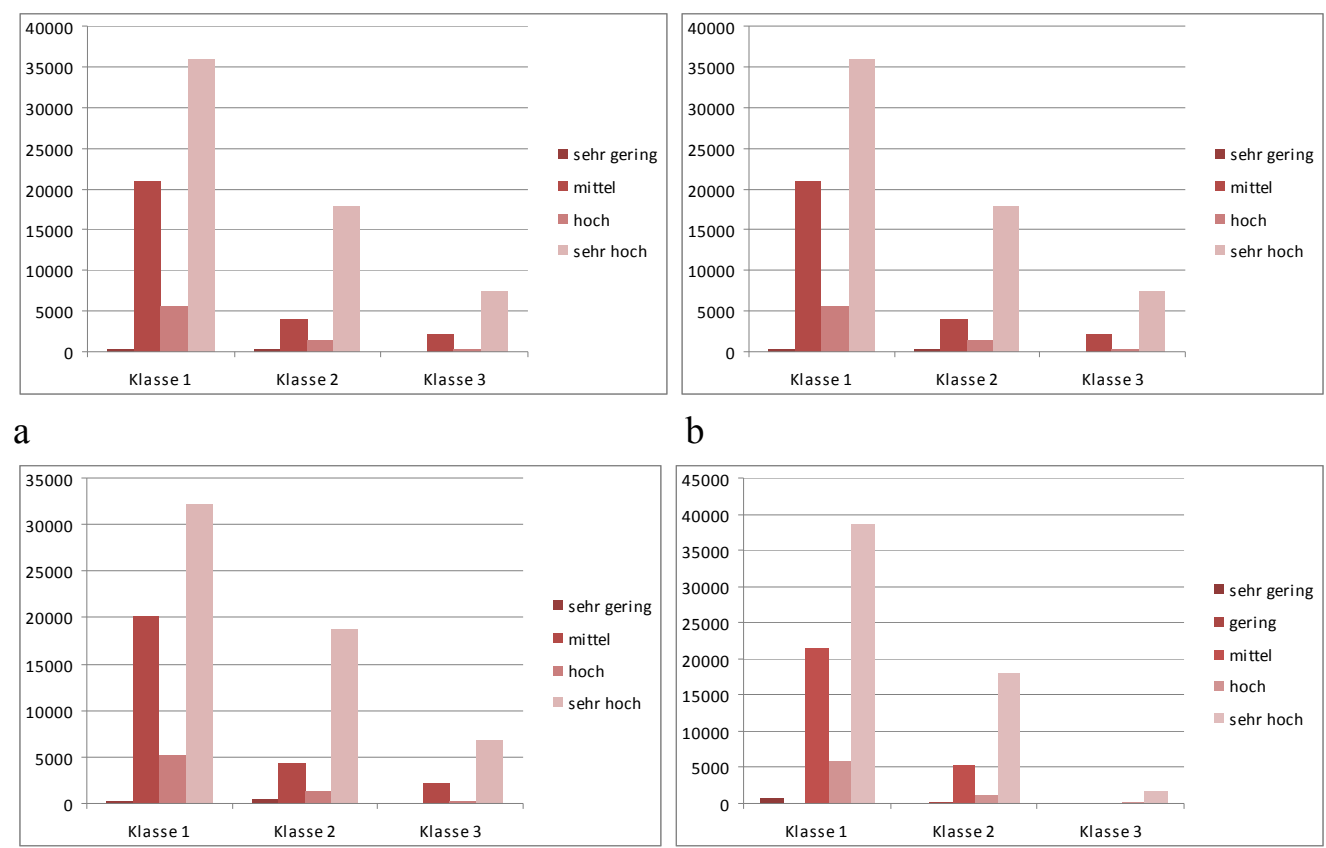

c

d

Abb. 79: Gegenüberstellung Besucherfrequenzen (Klassen 1 = geringe Besucherfrequenz, Klasse 2 = mittlere Besuchfrequenz, Klasse 3 = hohe Besucherfrequenz) und Vegetationssensibilität (gering bis hoch) durch Vergleiche der Streckenlänge der Wegabschnitte; Grafik a: Werktag 1999; Grafik b: Werktag 2012; Grafik c: Werktag unter Einbeziehung des Gewässerszenarios 1b; Grafik d: Werktag unter Einbeziehung des Gewässerszenarios $1 b$ und einer flächigen Ausprägung der Vorlandszenarien.

\subsubsection{Bewertung des Einflusses einer steigenden Erholungsnutzung auf die Vegetationstypen entlang der Wege in der Lobau}

Im zweiten Projektjahr wurde von der Gruppe Vegetationsökologie der Faktor Vegetationstyp bearbeitet. Neben der Verwendung bestehender Datengrundlagen aus dem Projekt OptimaLobau wurde die gestellte Aufgabe auch durch Freilanderhebungen des Projektteams, durch Diplomarbeiten und einer Lehrveranstaltung erfüllt. Ziel der Erhebungen war es, einen Überblick über die aktuellen Vegetationsverhältnisse der Lobau zu geben und die B-Diversität dieser Aulandschaft im Bereich der Wege abzubilden. Mittels Vegetationsaufnahmen auf objektiv ausgewählten Flächen wurden Informationen zu den verschiedenen Vegetationstypen gesammelt, die auch zur Evaluierung der Modle der agentenbasierten Analyse dienen werden (Aichhorn \& Paukovits, 2010).

\subsubsection{Daten und Methoden}

Mit Methoden aus dem Bereich der digitalen Raumanalyse besteht die Möglichkeit, Stichprobenpläne im Vorlauf zur Feldaufnahme zu entwerfen, die eine objektive Auswahl der Aufnahmeflächen erlauben oder zumindest Mischformen aus objektiver Übersichtswahl und subjektiver Detailwahl ermöglichen, welche mehr an Sicherheit für statische Auswertungen und Repräsentativität liefern.

Die Methode der stratifizierten, randomisierten Stichprobenauswahl (Mühlenberg, 1989) ist eine für die Stichprobenwahl für vegetationsökologische Untersuchungen adäquate Methode. Dabei wird das Gebiet, welches durch eine Stichprobe zu erfassen ist, in relativ homogene Untereinheiten unterteilt, indem verschieden vegetationsökologisch wirksame Faktoren - wie im vorliegenden Fall Biotoptyp und Wegetyp - verwendet werden. Durch die Stratifizierung des Untersuchungsgebietes kann die Grundgesamtheit in Teilmengen aufgelöst werden. Durch diesen Ansatz wird die Stichprobe auf ein bewältigbares Maß reduziert und die Aufnahmen stehen dann repräsentativ für das ganze Stratum.

Für die Umsetzung des gewählten methodischen Ansatzes war eine Überarbeitung der vorliegenden Biotoptypenkarte der MA22 nötig, da die Anzahl der Biotoptypen aus Gründen der Operationalisierung des gewählten Ansatzes reduziert werden mussten (Tab. 31). Die nach dem Biotoptypenkatalog des UBA beschriebenen 45 unterschiedlichen Biotoptypen in der Lobau wurden auf 15 aggregierte Typen zusammengefasst. Die so gebildete Biotoptypenkarte diente nicht nur als 
Basis für das Sampling - Design sondern auch für die Bildung einer flächendeckenden, grobskalierten Vegetationskarte des Untersuchungsgebietes.

\begin{tabular}{|l|l|}
\hline \multicolumn{1}{|c|}{ Agg. Typ } & \multicolumn{1}{c|}{ Biotoptyp } \\
\hline $\begin{array}{l}\text { Wald } \\
\text { dynamisch }\end{array}$ & Edellaubbaumdominierter Ufergehölzstreifen, Grauerlenauwald, Schwarzpappelauwald, \\
\hline & Silberpappelauwald, Schwarzerlen-Eschenauwald, Weidenauwald \\
\hline Wald stabil & Eichen-Ulmen-Eschen-Auwald, Laubbaumfeldgehölz aus standortstypischen Baumarten \\
\hline Gebüsch & Baumhecke, Gebüsche frischer Standorte, Hartriegelgebüsch, Holundergebüsch, \\
\hline & $\begin{array}{l}\text { Lavendelweiden-Sanddorngebüsch, Neophytengebüsch, Strauchmantel trocken-warmer } \\
\text { Standorte, }\end{array}$ \\
\hline Vorwald & thermophiles Trockengebüsch tiefgründiger Standorte \\
\hline Feldgehölz & Vorwald \\
\hline Forst A & Feldgehölz aus standortsfremden Baumarten \\
\hline & Ahornforst, Eschenforst, junge Laubbaumaufforstung, Silberpappel- und Weidenforst \\
\hline Forst B & Laubbaummischforst aus einheimischen Baumarten \\
\hline & Hybridpappelforst, Laubbaumforst aus sonstigen nichtheimischen Arten, \\
\hline & Nadelbaummischforst aus einheimischen Baumarten, Robinienforst, Rotföhrenforst, \\
\hline Park & Schwarzföhrenforst, Ufergehölzstreifen auf anthropogen überformten Standort \\
\hline Brache & Altbaumbestand in Park und Garten \\
\hline & $\begin{array}{l}\text { artenarme Ackerbrache, frische Grünlandbrache nährstoffreicher Standorte der } \\
\text { Tieflagen, }\end{array}$ \\
\hline & frische basenreiche Grünlandbrache nährstoffarmer Standorte der Tieflagen, \\
\hline Acker & kontinentale basenreiche Halbtrockenrasenbrache \\
\hline Hochstauden & intensiv bewirtschafteter Acker, Wildacker \\
\hline & Brennnesselflur, Ruderalflur frischer Standorte mit offener Pioniervegetation, \\
\hline Grünland & stauden- und farndominierte Schlagflur \\
\hline Neophytenflur & $\begin{array}{l}\text { frische, artenreiche Fettwiese der Tieflagen, Intensivweide der Tieflagen, } \\
\text { Überschwemmungswiese }\end{array}$ \\
\hline Magerwiese & Neophytenflur \\
\hline Trockenrasen & Karische basenreiche Magerwiese der Tieflagen \\
\hline & Karbonat-Schottertrockenrasen, kontinentaler basenreicher Mäh-Halbtrockenrasen \\
\hline ia Aggen &
\end{tabular}

Tab. 31: Aggregationstypen der in der Lobau beschriebenen Biotoptypen

Eine modellierte Vegetationskarte wurde auf Basis der Hauptbaumarten aus der Waldinventur (MA 49) geschaffen, wobei pro Waldparzelle eine grobe vegetationsökologische Analyse versucht wurde. Der gesamte Datensatz umfasste 740 „Pseudoaufnahmen“ = „Waldparzelle der Waldkarte“. Diese „Pseudoaufnahmen“ wurden für die Erzeugung einer Vegetationskarte mit Analyseverfahren bearbeitet, die eigentlich für echte Vegetationsaufnahmen geschaffen wurden. Die Datensätze, die in der Regel zwei bis vier Hauptbaumarten umfassen, konnten mit TWINSPAN (Hill, 1979) in acht als sinnvoll erscheinende Klassen aufgeteilt werden. Die erarbeiteten punktuell vorliegenden Vegetationsdaten wurden durch die Zuweisung zu Segmenten einer CIR-Bilddatenanalyse (Programme Ecognitionin und Erdas Imagine) in die Fläche extrapoliert. Durch die Kombination (Abb. 80) der Ergebnisse einer modellierten Vegetationskarte und der auf Felderhebungen basierenden Biotoptypenkarte wurde eine Karte geschaffen, die die Vegetationsverhältnisse der Lobau auf einem sehr hohen Aggregationsniveau abgebildet. Diese Vegetationskarte bildet die Grundlage des Bewertungskriteriums „Vegetationstyp“ in der agentenbasierten Modellierung der Wegenutzung der BesucherInnen. 


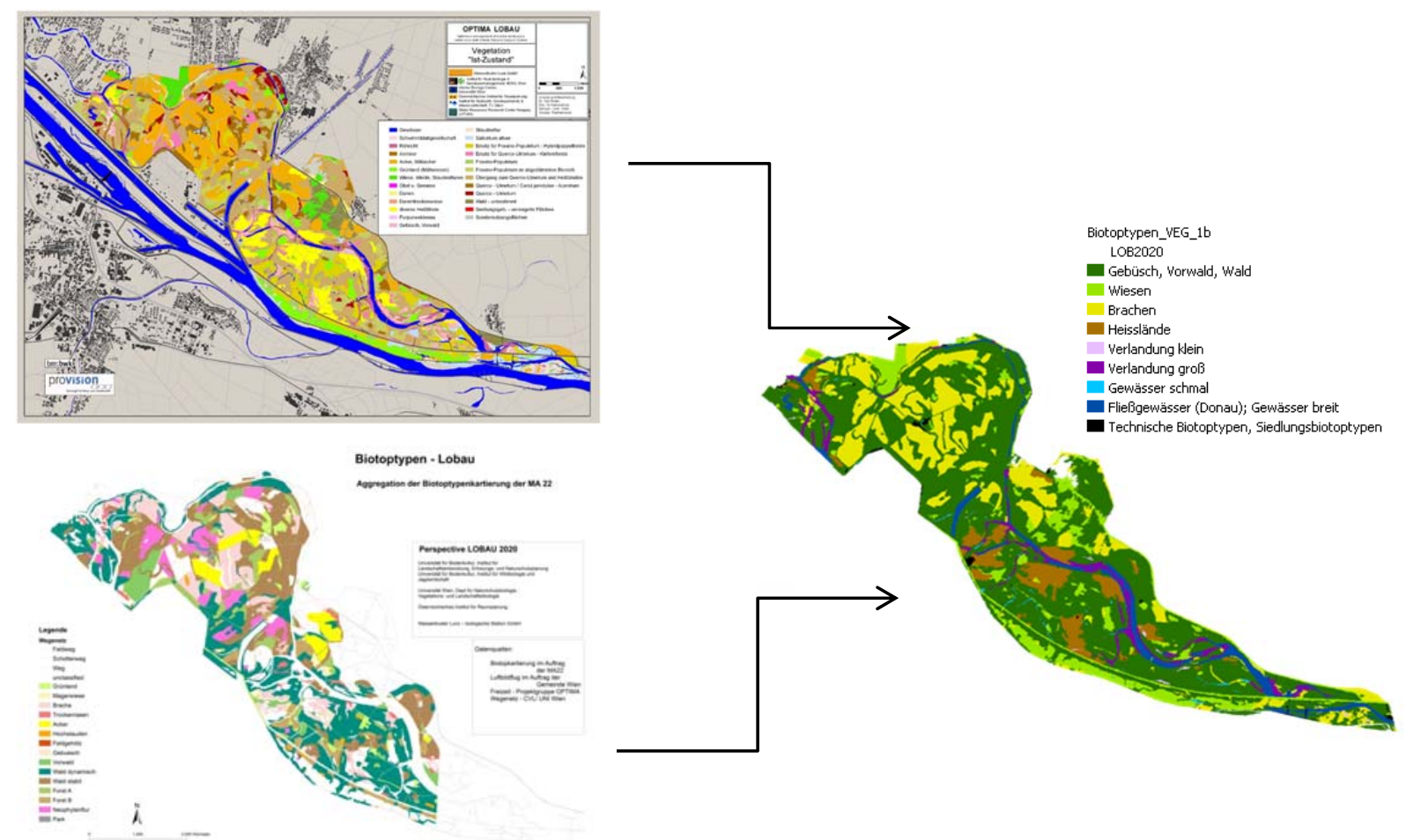

Abb. 80: Darstellung der Kombination einer modellierten Vegetationskarte und der Biotoptypenkarte zu einer grobskalierten Vegetationskarte

Die als Linienelemente vorliegenden Daten zu den Wegen wurden mit 20 Meter links und rechts gepuffert und mit der Biotoptypenkarte der Lobau verschnitten. Durch diese Verschneidung wurden 54 unterschiedliche Kombinationstypen (= Stratum) aus Wegetyp und Biotoptyp gebildet. Durch ein randomisiertes Verfahren wurden je vier Flächen pro Stratum gewählt (Abb. 81).

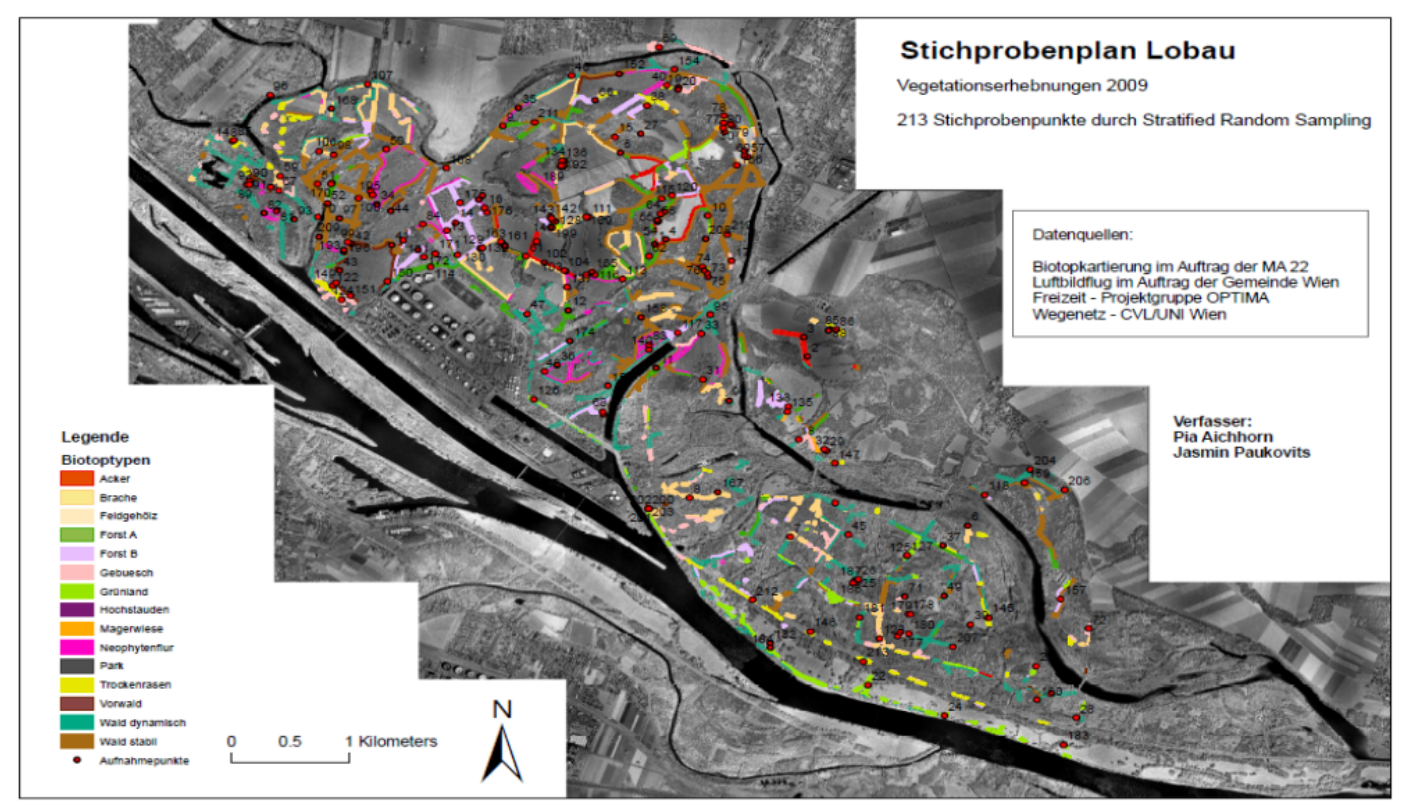

Abb. 81: Stichprobenplan der Freilanderhebungen im Sommer 2009 mit 213 ausgewiesenen Erhebungsbereichen 
Geländeerhebung

Für die Vegetationsaufnahmen wurden pro Wegabschnitt Transekte normal zum Weg gelegt, um Gradienten ausgehend vom Weg in Richtung des jeweiligen Biotoptyps sichtbar zu machen. Es wurden jeweils auf derjenigen Seite des Weges Vegetationserhebungen durchgeführt, auf der sich der Stichprobenpunkt laut Stichprobenplan befand. Handelte es sich rechts und links vom Weg um heterogene Biotoptypen, so wurde die gegenüberliegende Seite des Weges meist ebenfalls aufgenommen. Die Transekte setzten sich jeweils aus einem Rechteck (Wegrand) und einem darauf anschließenden Quadrat (Biotop) zusammen (Abb. 82), wobei die Seitenlänge des Quadrates laut Minimum-Areal des entsprechenden Biotoptyps festgelegt war.

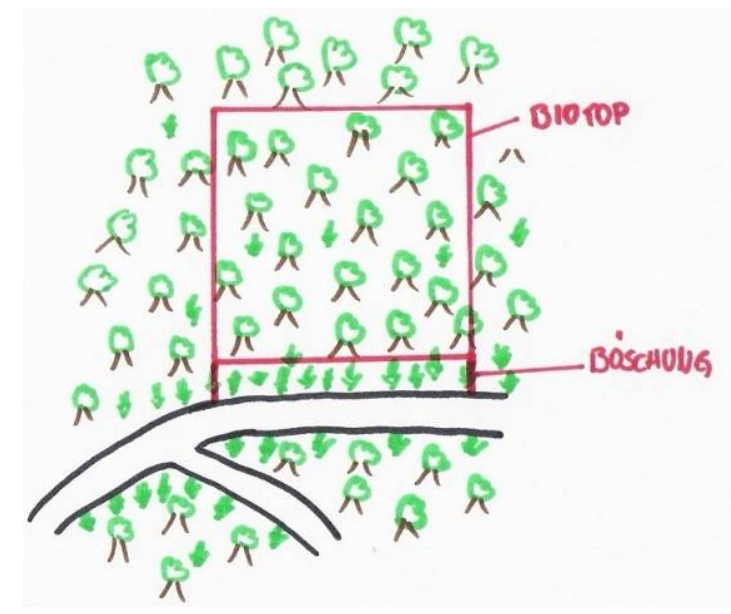

Abb. 82: Skizze einer Erhebungsfläche

Das Minimum Areal ist die Mindestfläche für das Vorkommen eines nach Struktur und Artenzahl typischen Bestandes einer Pflanzengesellschaft und ist oft Gegenstand vieler Diskussionen. Deshalb werden für die praktische Geländearbeit meist nur allgemeine Erfahrungswerte benutzt (Dierschke, 1994) (Tab. 32). Die Breite des Rechteckes richtete sich nach der tatsächlichen Breite des Wegrandes (meist zwischen 0,5 und $1 \mathrm{~m}$ ) und die Länge des Rechteckes wurde der entsprechenden Seitenlänge des jeweiligen Biotoptyps (Quadrat) angepasst. Die Abgrenzung der Aufnahmefläche erfolgte subjektiv. Die Vegetationsaufnahmen sollten auf möglichst homogenen Flächen mit einheitlichen Standortbedingungen und einheitlicher Vegetation erstellt werden. Je nach Art des Biotops wurden folgende Richtwerte, angelehnt an Dierschke (1994), aus der Tabelle entnommen.

\begin{tabular}{|l|c|c|}
\hline \multicolumn{1}{|c|}{ Biotoptyp } & $\begin{array}{c}\text { Flächengröße } \\
\text { (Quadrat) }\end{array}$ & $\begin{array}{c}\text { Länge des Wegrandes } \\
\text { (Rechteck) }\end{array}$ \\
\hline Wald & $100-400 \mathrm{~m} 2$ & ca. $10 \mathrm{~m}$ \\
\hline $\begin{array}{l}\text { Gebüsche, Feldgehölz, Vorwald, ruderale } \\
\text { Vegetation }\end{array}$ & $25-100 \mathrm{~m} 2$ & ca. $5 \mathrm{~m}$ \\
\hline $\begin{array}{l}\text { Magerwiese, Trockenrasen, Hochstauden, } \\
\text { Neophytenfluren }\end{array}$ & $10-25 \mathrm{~m} 2$ & ca. $5 \mathrm{~m}$ \\
\hline
\end{tabular}

Tab. 32: Größe der Aufnahmeflächen

Erfassung verschiedener Flächenparameter

In jedem Transekt wurden außer der Artenzusammensetzung und Artmächtigkeit der Vegetation noch andere Flächenparameter wie z.B. offener Boden [\%] oder litter (Bestandesabfall der Vegetation) [\%] erhoben (Abb. 83). 


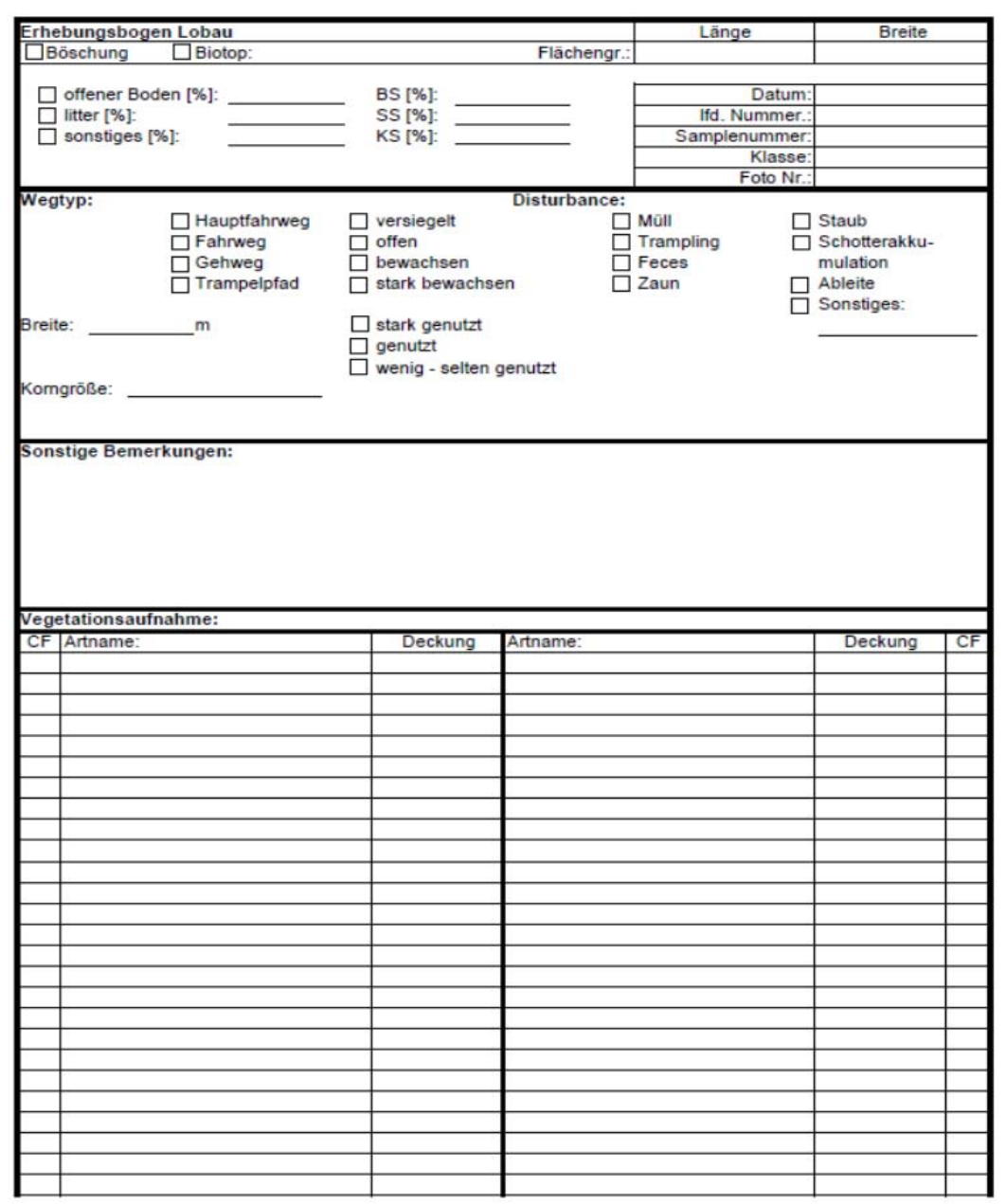

Abb. 83: Erhebungsblatt für die Freilanderhebungen

\section{Wegtypen}

Im Nationalpark Lobau wurden vier verschiedene Wegtypen dokumentiert:

- Hauptfahrweg

- Fahrweg

- Gehweg

- Trampelpfad

Außerdem wurde der Versiegelungs- bzw. Benutzungsgrad der befahrenen oder begangenen Wege innerhalb der Lobau festgehalten und die Breite der jeweiligen Wege geschätzt.

Anthropogene Störung

Um die anthropogene Störung innerhalb der Transekte festzustellen, wurden folgende Disturbanceeingriffe sowohl am Wegrand als auch im angrenzenden Biotoptyp notiert:

- Müll

- Staub

- Trampling

- Schotterakkumulation

- Feces

- Ableite

- Zaun

- Sonstiges 


\section{Korngröße}

Unter Körnung des Bodens versteht man die gewichtsmäßige Zusammensetzung des Bodens aus verschiedenen Fraktionen von mineralischen Einzelkörnern mit bestimmtem Korndurchmesser (Braun-Blanquet, 1964).

Zur Bestimmung der Korngrößenfraktion wurde die Fingerprobe angewendet. Dabei wird feuchter Boden zwischen den Fingern gerieben. Grober Sand fühlt sich grobkörnig an, feiner Sand samtig, Schluff ist glatt und nicht haftend und Ton klebt an den Fingern. Dieser Test ist eine schnelle Methode zur Bestimmung der Körnung und eignet sich besonders für die Arbeit im Gelände (Wild, 1995).

\subsubsection{Ergebnisse}

In der Lobau wurden 375 Vegetationsaufnahmen (beinhalten sowohl Wegrand- als auch Biotoptypaufnahmen) erstellt, die 13 verschiedenen Gesellschaften zugeordnet werden konnten. Die restlichen vier Gruppen sind pflanzensoziologisch nicht zu klassifizieren, da es sich um anthropogen geschaffene Biotope (Aesculus hippocastanum-Allee, Tilia cordata -Allee, Medicago sativa-Acker und Hordeum vulgare-Acker) handelt (Tab. 33).

\begin{tabular}{|l|l|}
\hline Fraxino-Ulmetum caricetosum albae & Tanaceto-Arrhenatheretum \\
\hline Fraxino-Populetum typicum & Lolietum perennis \\
\hline Pruno-Ligustretum typicum & Teucrio botryos-Andropogonetum ischaemii \\
\hline Echinochloa-setarietum pumilae & Onobrychido viciifoliae-Brometum \\
\hline Setarietum viridis-verticillatae & Tilia cordata-Allee \\
\hline Calamagrostis-epigejos-Gesellschaft & Hordeum vulgare-Acker \\
\hline Elymus repens-Gesellschaft & Medcago sativa-Acker \\
\hline Solidago-gigantea Gesellschaft & Aesculus hippocastanum-Allee \\
\hline Pastinaco-Arrhenatheretum & \\
\hline
\end{tabular}

Tab. 33: Syntaxone und klassenlose Vegetationsformationen beschrieben auf Basis des 375 Aufnahmen umfassenden Samples

Bei genauerer Betrachtung des Datenmaterials wurde die Ruderalvegetation besonders an stark genutzten Hauptfahrwegen bzw. Fahrwegen ausgewiesen. Die Ruderalvegetation ist durch ständige anthropogene Eingriffe bedingt. Die Eingriffe erfolgen hier nicht systematisch bzw. regelmäßig, sondern mehr oder weniger zufällig. Obgleich es den Anschein hat, als wäre das Auftreten der Arten an solchen ruderal gestörten Stellen, wie z.B. Böschungen, zufällig, lassen sich auch hier, wie bei der übrigen Vegetation, bestimmte Gesetzmäßigkeiten finden. Ein neugebildeter Ruderalstandort wird meist von einjährigen Pflanzen (Sommerannuellen) besiedelt. Im darauffolgenden Jahr kommen mehr winterannuelle Pflanzen zur Vorherrschaft. Diese Arten keimen im Herbst, überwintern als Jungpflanzen, blühen, fruchten und sterben in der darauffolgenden Vegetationsperiode. Bei weiterer ungestörter Entwicklung kommen immer mehr ausdauernde Gewächse auf. Auch verschiedene ausdauernde Gräser besiedeln bald diese Standorte, gefolgt von Bäumen und Sträuchern (Hübl, 1974).

Die in dieser Arbeit ausgewiesenen Ruderalgesellschaften, vor allem die Aufnahmen der Elymus repens-Gesellschaft und die Aufnahmen der Lolietum perennis-Gesellschaft, sowie auch Aufnahmen des Tanaceto-Arrhenatheretums, bilden durch sogenannte „Betrittpflanzen“ artenarme Gesellschaften. Sowohl die starken Bodenverdichtungen als auch die mechanische Beanspruchung erschweren hier das Pflanzenleben. Deshalb besitzen „Betrittpflanzen“ Eigenschaften, die mechanische Beschädigung möglichst verhindern. Beispiele für Anpassungserscheinungen von „Betrittpflanzen“ sind die Kleinheit der gesamten Pflanze, die Kleinheit der Blätter, gutes Regenerationsvermögen, Festigkeit der Gewebe, Biegungsfähigkeit, Bildung von Ausläufern und dadurch Besiedlung von Stellen, wo die Keimlinge nicht aufkommen könnten (Hübl, 1974).

Häufig aufgenommene Betrittpflanzen im Untersuchungsgebiet sind Poa annua, Lolium perenne, Polygonum aviculare, Plantago major, Sclerochloa dura und Trifolium repens. 
Wegtypen der Oberen Lobau

In der Oberen Lobau tritt der Wegtyp „Fahrweg“ mit $35 \%$ an erste Stelle auf, gefolgt vom „Gehweg“ mit $32 \%$ und vom „Hauptfahrweg“ mit $22 \%$. Die „Trampelpfade“ nehmen mit $11 \%$ die letzte Stellung ein (Abb. 84). Die Obere Lobau ist von einem dichten Wegenetz durchzogen.

\section{Wegtypen der Oberen Lobau}

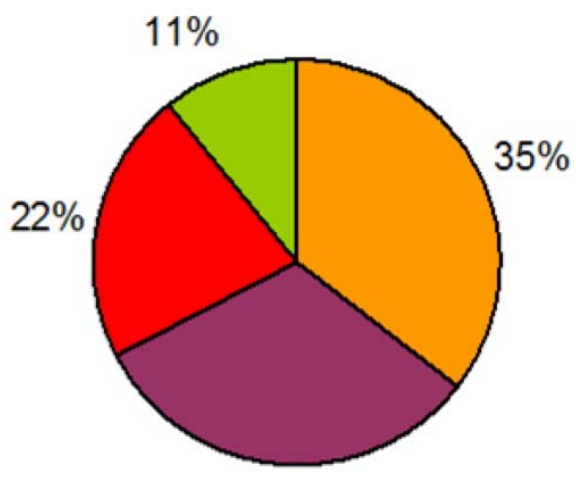

\begin{tabular}{l}
\hline Fahrweg \\
$\square$ Gehweg \\
$\square$ Hauptfahrweg \\
$\square$ Trampelpfad
\end{tabular}

$32 \%$

Abb. 84: Prozentuelle Anteile der aufgenommenen Wegetypen der Oberen Lobau

Wegtypen der Unteren Lobau

In der Unteren Lobau liegt der „Fahrweg“ mit $44 \%$ ebenfalls an erster Stelle, gefolgt vom „Hauptfahrweg“ mit $30 \%$. Im Gegensatz zur Oberen Lobau tritt der „Gehweg“ mit einem geringen Anteil von nur $11 \%$ auf. „Trampelpfade“ sind mit einem Anteil von $15 \%$ vertreten (Abb. 85).

\section{Wegtypen der Unteren Lobau}

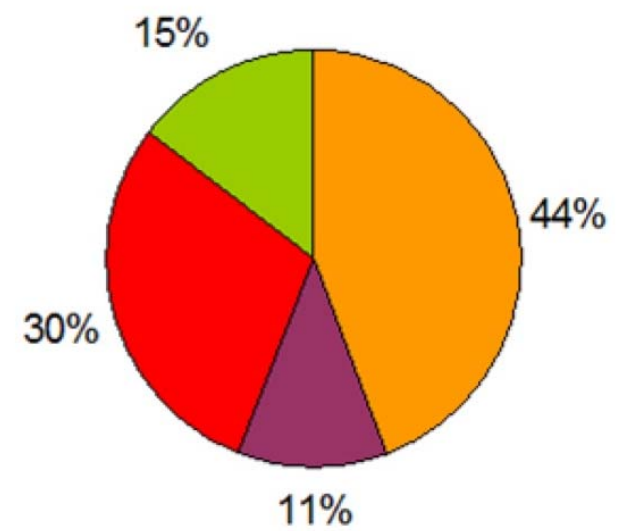

$$
\begin{aligned}
& \square \text { Fahrweg } \\
& \square \text { Gehweg } \\
& \square \text { Hauptfahrweg } \\
& \square \text { Trampelpfad }
\end{aligned}
$$

Abb. 85: Prozentuelle Anteile der aufgenommenen Wegetypen der Unteren Lobau

In der Unteren Lobau wurden mehr Trampelpfade erfasst, weil hier verhältnismäßig wenig markierte Wanderwege liegen. Vielerorts sind in der Unteren Lobau Trampelpfade ausgebildet, die in der Nähe von Wildniszonen liegen und von BesucherInnen nicht betreten werden sollen. 


\section{Disturbance}

In der Oberen Lobau ist ein höherer anthropogener Störungseinfluss zu verzeichnen (Abb. 86). Dies ist vor allem in den Bereichen „Müll“, „Trampling“, „Staub“ und „Feces“ zu beobachten. Eindeutig ersichtlich ist dies am Beispiel von „Müll“. Während in der Unteren Lobau nur $13 \%$ vorliegen, sind in der Oberen Lobau $29 \%$ der gesamten aufgenommenen Wegränder und Biotope als verschmutzt gewertet worden. Auffällig ist, dass die Disturbancekategorie „Sonstiges“ in der Unteren Lobau eindeutig überwiegt. Häufig auftretende Beispiele für die Kategorie „Sonstiges“ sind Mahd, Fahrzeugspuren von LKWs oder Traktoren, Deponie von organischem Material. Ebenfalls im Bezug auf die „Schotterakkumulation“ ist die Untere Lobau mit einem höheren Prozentanteil vertreten. Es liegen geringe Prozentsätze der Kategorie „Feces“ von $4 \%$ in der Oberen Lobau und $3 \%$ in der Unteren Lobau vor.

\section{Disturbancevergleich zwischen Oberer und Unterer Lobau}

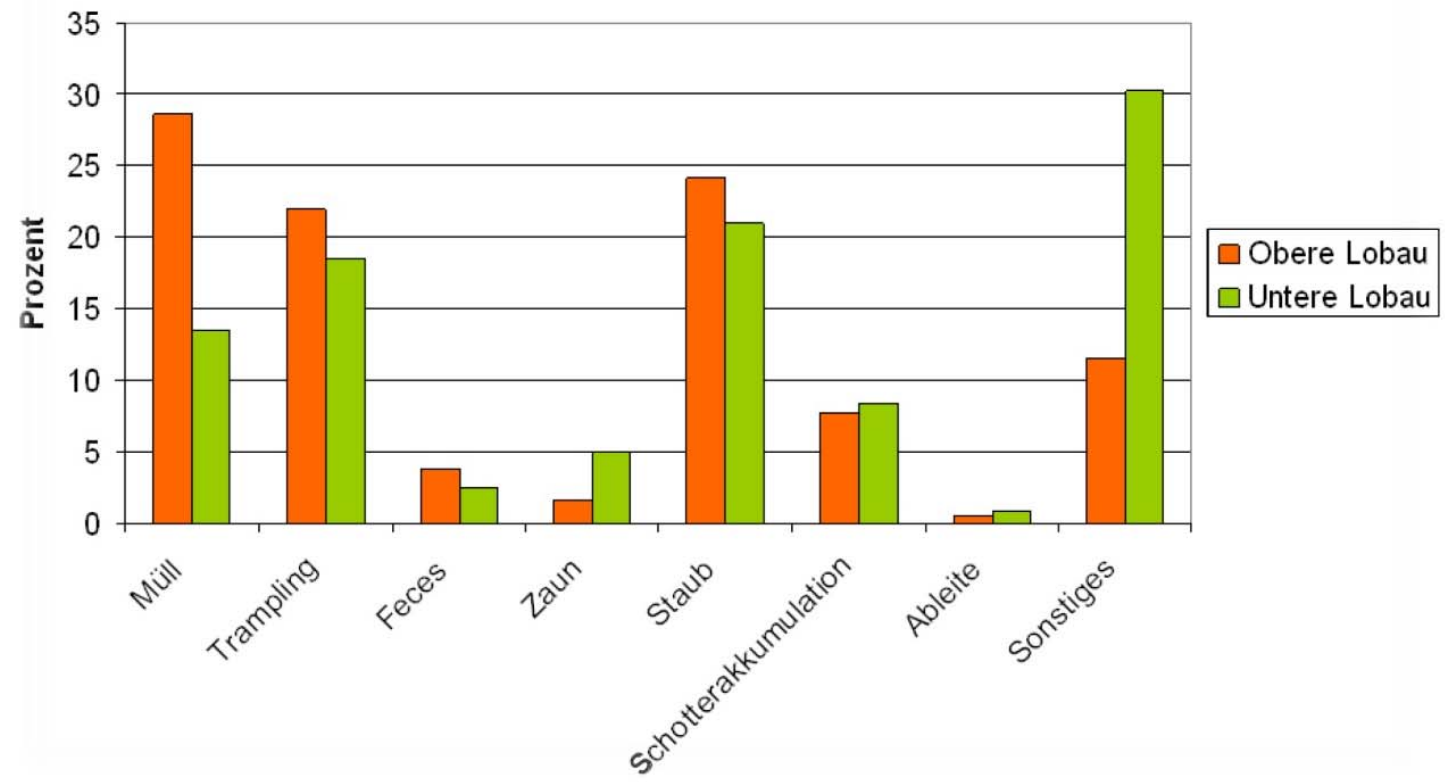

Abb. 86: Auftretende Disturbance in der Oberen und Unteren Lobau

\subsubsection{Diskussion}

Betrachtet man die Biotoptypenzusammensetzung, so treten z.B. in der Oberen Lobau vermehrt Äcker, Forste, Neophytenfluren und anthropogen geschaffene Bereiche wie Parks, Freibadebereiche, Rastund Spielplätze auf. Ebenfalls herrscht in der Oberen Lobau der stabile Wald vor, wobei der dynamische Teil der Wälder in der Unteren Lobau überwiegt. Die naturnahen Trockenrasen und Heißländen sind ebenfalls nur in der Unteren Lobau anzutreffen.

Ob die Wegränder sich signifikant von den daran anschließenden Biotoptypen unterscheiden, weil sie, wie angenommen, vom Menschen deutlich mehr beeinflusst werden, kann eher verneint werden. Oft war es der Fall, dass Wegrand und dazugehöriger Biotoptyp sich in ihrer Artenzusammensetzung nicht wesentlich unterschieden.

Aber typische Trittgesellschaften wie z.B. Lolietum perennis zeigen sehr wohl den menschlichen Einfluss auf die Vegetation in der Lobau. Die Annahme, dass die Wegränder naturferner sind, weil anthropogen intensiver beeinflusst, konnte nicht bewiesen werden. Zum Teil war es sogar der Fall, dass die Wegränder naturnaher erschienen als die angrenzenden Biotoptypen. 


\subsection{Kriterien Gewässerökologie}

\subsubsection{Grundlagen der Bewertung}

\subsubsection{Einleitung}

Die Auswirkungen der in den verschiedenen Szenarien festgehaltenen möglichen Entwicklungen und Nutzungen in der Unteren Lobau auf die Gewässer, von deren optischen Eindruck auf die BesucherInnen bis hin zur ökologischen Funktionsfähigkeit im Fluss/Au-System Donau, werden über die Modellierung des aquatischen Primärproduktionspotentials bewertet. Aquatische Primärproduzenten sind gerade in hoch produktiven Systemen, wie zum Beispiel in Augebieten von Flüssen, von entscheidender Bedeutung für das Kohlenstoff-Angebot im Nahrungsnetz und den Abbau von Nährstoffen im Fluss und tragen zur morphologischen Entwicklung der Gewässer im Hinblick auf Verlandungsprozesse bei. Die Intensität der Primärproduktion (Trophie) und die Hydromorphologie eines Gewässers kontrollieren maßgeblich die Entwicklung der Makrophyten und der Wasserqualität und haben somit starken Einfluss auf das Erscheinungsbild eines Gewässers und die Wahrnehmung durch BesucherInnen.

Auf Basis von vorhandenen ökologischen Modellen aus dem Projekt „OptimaLobau“, können der Makrophyten-Deckungsgrad und die Phytoplanktonbiomasse für die verschiedenen Szenarien im Projektgebiet modelliert werden. Das bietet einerseits die Möglichkeit, Eingangsparameter für andere Modelle (Besucherfrequenzanalysen), wie das Aussehen von Gewässern (Schilfbedeckung - freie Wasserfläche) für Besucher festzulegen, andererseits werden daraus Kriterien zur Bewertung der Szenarien (Risikoanalyse) abgeleitet.

Basierend auf einem zweidimensionalen, numerischen Oberflächenströmungsmodell, das die komplexe Strömungssituation die durch die rückströmende Anbindung aus der Donau in der Lobau entsteht, abbilden kann, wurden die ökologischen Modelle so aufgebaut, dass sie flächendeckende Darstellungen der modellierten Parameter im Untersuchungsgebiet ermöglichen. Artenanzahl, Dichte und Deckungsgrad von Makrophyten und ihre Lebensformen wurden modelliert und mit der Modellierung der Phytoplanktonbiomasse gekoppelt.

Daraus abgeleitet werden folgende Kriterien:

\section{- Auswirkungen des Besuchereinflusses auf die Gewässervegetation}

Makrophyten sind Indikatoren für die Wasserqualität, da sie wesentlich zur Produktion von Sauerstoff im Gewässer beitragen. Weiters stellen sie eine primäre Quelle von organischem Material in Altarmen dar und haben einen großen Effekt auf die physikalische Umwelt der Gewässer, wie z.B. auf die Sedimentstabilisation, Lichtverhältnisse und Strömung. Ziel der Bearbeitungen ist es, das im Rahmen des Projekts Optima Lobau entwickelte statistische Makrophytenmodell auf die Gegebenheiten von erhöhtem Besucherdruck zu adaptieren. Dabei wird der Einfluss von Badenutzung auf die Makrophytenverteilung und -zusammensetzung untersucht und in das Modell eingearbeitet. Die Modellergebnisse können als Entscheidungskriterien für das agentenbasierte Modell sowie die Modellierung der Phytoplankton-Biomasse einfließen.

- Auswirkungen des erhöhten Besucherdrucks auf den Trophiegrad von Gewässern:

Die trophischen Verhältnisse in Gewässern werden durch die Nährstoffverfügbarkeit und den Gehalt an planktischer Biomasse bestimmt. Im Rahmen der Bearbeitungen wird das für Optima Lobau entwickelte Phytoplanktonmodell auf den erhöhten Nutzerdruck adaptiert. Beeinflussungen sind durch eine Veränderung der Makrophytendeckung (die einen wesentlichen Faktor für die Phytoplanktonentwicklung darstellt) und den erhöhten Nährstoffeintrag durch Badenutzung zu erwarten.

Aus dem Modell abgeleitete Indikatoren werden für die Bewertung der Szenarien, als Zeiger für die ökologische Funktionsfähigkeit der Gewässer verwendet. 


\subsubsection{Modellaufbau:}

\subsection{Makrophyten}

Die Makrophyten wurden in die Lebensformen Hydrophyten („echte“ Wasserpflanzen) und Helophyten (Uferpflanzen) eingeteilt.

Als Indikatoren wurden folgende Parameter berechnet:

- Menge Makrophyten gesamt sowie Menge der Hydrophyten: Die Makrophyten sind Indikatoren für die Wasserqualität, da sie wesentlich zur Produktion von Sauerstoff im Gewässer beitragen. Weiters haben sie einen großen Effekt auf die physikalische Umwelt der Gewässer und interagieren mit Phytoplankton (Licht-, Nährstoffkonkurrenz).

- Fläche der Helophyten: Helophyten sind ein Verlandungszeiger und bieten die Möglichkeit, die Entwicklung der Gewässer darzustellen, sind aber auch ein wesentliches Habitat für verschiedene Lebewesen, wie z.B. Vögel.

Im Rahmen der Modellentwicklung wurde die Beziehung zwischen hydraulisch modellierbaren Parametern (Wasserfläche, Wassertiefe, Fließgeschwindigkeit) und der Entwicklung von Makrophyten-Gemeinschaften und der Artenzahl analysiert. Die Makrophytendaten stammen aus Kartierungen nach Kohler aus verschiedenen Projekten („Dotation 1996 und 1998“, „Life Projekt 2000-2001“ und „Regelsbrunn: Gewässervernetzung 2000“), die in der Lobau durchgeführt wurden. Die Methode nach Kohler ist eine Schätzmethode für die Pflanzenmenge in den Gewässern. Bei dieser Methode wird das Untersuchungsgebiet in Abschnitte unterteilt und pro Abschnitt die Vegetationsmenge nach einer fünfstufigen Skala geschätzt (Kohler \& Janauer 1995). Für die statistische Analyse wurde der Kohler-Wert pro Art nach der Funktion $\mathrm{f}(\mathrm{x})=\mathrm{ax}^{3}$ umgerechnet (Janauer \& Heindl 1998). Die Summe der umgerechneten Kohler-Werte pro Abschnitt für die gesamten Makrophyten, für die Hydrophyten, für die für den Naturschutz relevanten Arten und für die Helophyten wurden berechnet.

Die Modellierung wurde mit Hilfe einer multiplen Regression durchgeführt (Tab. 34), bei der die

- Größe der Flachwasserbereiche (Bereiche tiefer als $1 \mathrm{~m}$-hydrologische Modellierung bei einem Donauabfluss von $3000 \mathrm{~m}^{3} / \mathrm{s}$ ) und die

- Strömungsgeschwindigkeit in den Abschnitten bei einem Abfluss der Donau von $4000 \mathrm{~m}^{3} / \mathrm{s}$ als Hauptfaktoren für die Makrophytenabundanz und die Artenzahl ermittelt wurden.

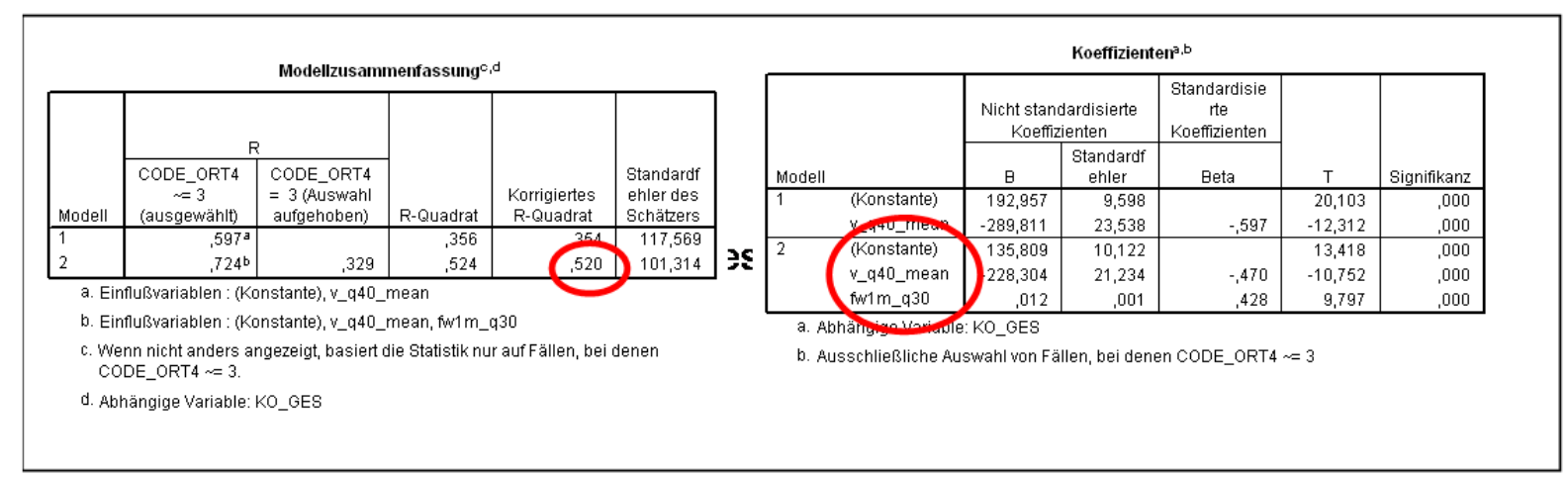

Tab. 34: Ergebnisse der multiplen linearen Regression für Makrophyten gesamt (Baart 2008).

Abb. 87 zeigt die gewässerinternen ökologischen Zusammenhänge und die Auswirkung der Hauptfaktoren auf die Makrophyten (Baart, 2008). 


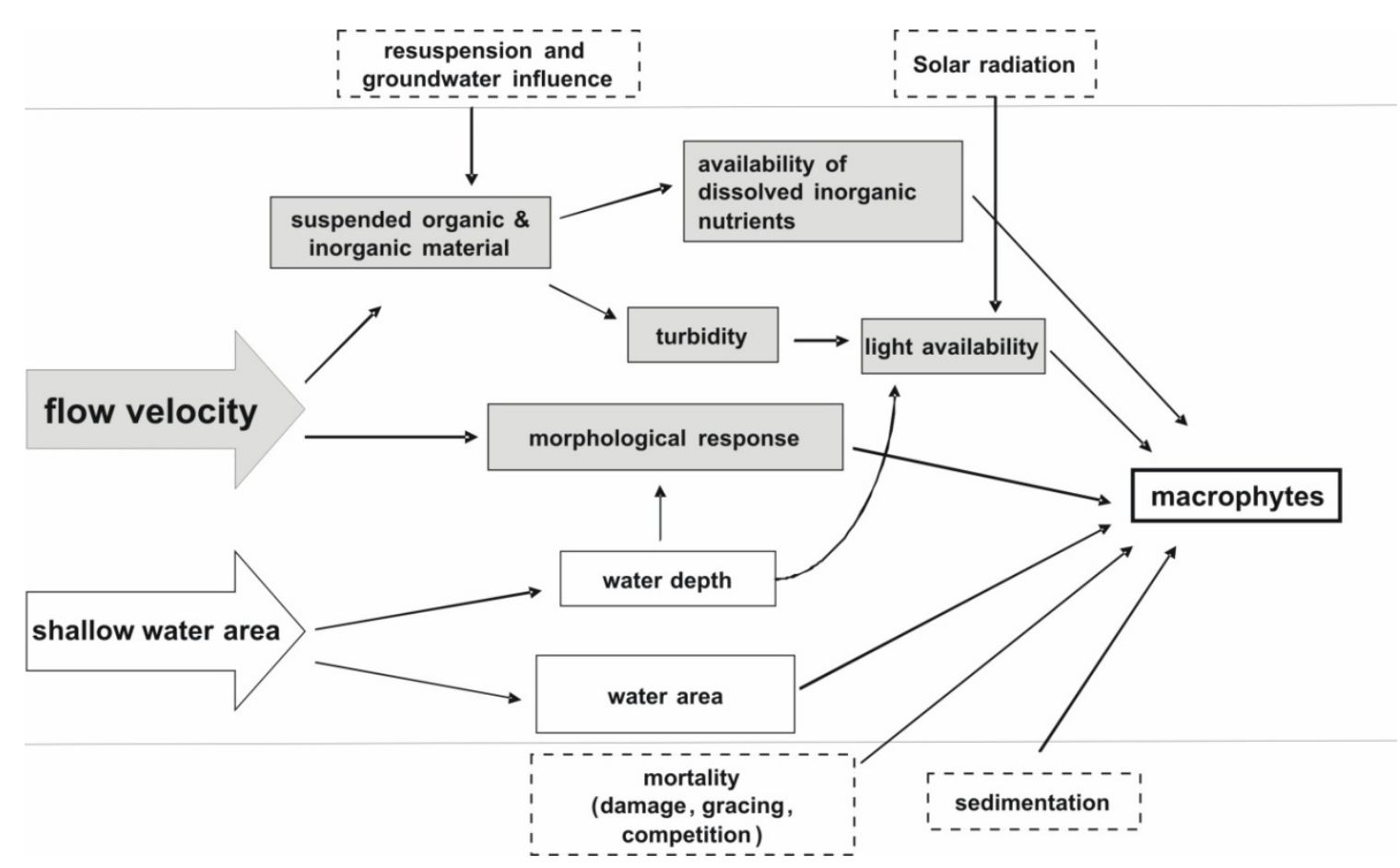

Abb. 87: Zusammenhang und Wirkung der Hauptfaktoren auf die Makrophytenverteilung (Baart, 2008).

Modelladaptierung:

Die Anpassung des vorhandenen Modells erfolgte durch eine Integration der verfügbaren Daten über Badenutzung in der Unteren Lobau (Kap. 4.1.3 Befragung des Wahlmodells).

Badenutzung führt zu einem Nährstoffeintrag (der in die Modellierung der PhytoplanktonPrimärproduktion eingeht) und zu mechanischer Zerstörung von Makrophyten.

Folgende Annahmen wurden dafür getroffen:

- Baden ist in den ausgewiesenen Badegewässern Panozzalacke, Dechantlacke und DonauOder-Kanal, sowie in Gewässern, die gut zugänglich sind (Wegnähe), deren Größe $2.500 \mathrm{~m}^{2}$ und deren mittlere Tiefe $>1 \mathrm{~m}$ übersteigt, möglich.

- In den ausgewiesenen Badegewässern werden im Umkreis der Liegeflächen (mit Einstiegstellen) submerse Makrophyten bis zu einer Tiefe von 1,5 m um $50 \%$ reduziert, an tieferen Stellen um $25 \%$.

- In den restlichen Gewässern, die eine Badenutzung ermöglichen, werden im Umkreis der Einstiegstellen $(10 \mathrm{~m})$ submerse Makrophyten bis zu einer Tiefe von 1,5 m um $30 \%$ reduziert, an tieferen Stellen um $15 \%$.

- Schilf und andere emerse Makrophyten werden kaum von Badegästen beeinträchtigt

Unter diesen Annahmen wurden mit der Software ArcGIS durch eine Verschneidung von Wegdaten (Wahlmodell), Luftbildern und digitaler Höhenmodelle die Bereiche abgegrenzt, wo eine Beeinträchtigung zu erwarten ist. Die Ergebnisse der Makrophyten-Modellierung wurden dem entsprechend adaptiert und als Grundlage für die Modellierung der Phytoplankton-Biomasse verwendet.

\subsection{Phytoplankton}

Aus vorhandenen Daten aus der Oberen Lobau, der Unteren Lobau und Regelsbrunn im Zeitraum von 1995 - 2006 (978 Datensätze von 33 Probestellen) wurden die Zusammenhänge zwischen abiotischen Faktoren und der Phytoplanktonbiomasse, ausgedrückt als mittlerer Chl-a-Gehalt für eine Vegetationsperiode, untersucht. Dazu wurde das Gewässersystem, basierend auf den hydrologischen Modellierungen, in Abschnitte eingeteilt, die in Bezug auf die mittlere Tiefe und den Anbindungsgrad homogen waren. Alle Berechnungen wurden auf GIS-Basis mit der Software ArcGIS 9.2 durchgeführt. Grundlagen waren die erarbeiteten Höhenmodelle und die hydrologischen Modellierungen. Mittels einer multiplen linearen Regression wurden die Zusammenhänge von Chl-a- 
Gehalt und den jeweiligen Faktoren ermittelt und die Anzahl der untersuchten Größen anhand des berechneten Erklärungswerts reduziert.

Als für den Chl-a-Gehalt in den Gewässern der Lobau wesentliche Faktoren wurden folgende ermittelt:

- Anteil der Flachwasserbereiche

Für diesen Indikator wurde der Anteil der Bereiche eines Abschnitts mit einer Tiefe kleiner als 0,5 m an der gesamten Wasserfläche bei einer Donau-Wasserführung von $2200 \mathrm{~m}^{3} \mathrm{~s}-1$ berechnet.

- Mittlere Hydrophytendeckung (Kohlerwert Hydrophyten)

Der Deckungsgrad an Hydrophyten wurde aus den Ergebnissen der oben dargestellten MakrophytenModellierungen erhalten, um die Konkurrenz von Phytoplankton und Makrophyten um Nährstoffe und Licht als Faktor miteinzubinden.

- Konnektivität (Tage der Anbindung an die Donau pro Jahr)

Die verwendeten Abschnitte wurden durch Verschneidung der Gewässerflächen bei Q3000 (aktuelle Grund- und Oberflächenwassermodellierungen kombiniert) mit Bereichen gleicher Konnektivität definiert. Der Anbindungsgrad der Gewässerbereiche entspricht dabei:

Q910 - 345 Tage im Jahr

Q2200 - 137 Tage im Jahr

Q3000 - 44 Tage im Jahr

Q4000 - 10 Tage im Jahr

Q5000 - 2 Tage im Jahr

Rest - 0 Tage im Jahr

Um den nichtlinearen Zusammenhang zu berücksichtigen, gingen die Werte der Konnektivität logarithmiert in die Analyse ein.

Für die multiple lineare Regression wurden Daten der Abschnitte aus dem Hauptgewässerzug in der Unteren Lobau, aus Regelsbrunn (stellvertretend für höhere Anbindungsgrade) und Nebengewässern verwendet.

\begin{tabular}{|l|c|c|c|}
\hline \multicolumn{1}{|c|}{ Modell } & Wert & Standardfehler & Signifikanz \\
\hline (Konstante) & 16,630 & 5,116 & 0,004 \\
\hline AntFWB & $-0,048$ & 0,041 & 0,025 \\
\hline KO_Hydr & $-0,053$ & 0,012 & 0,0002 \\
\hline ln con & 1,682 & 0,979 & 0,01 \\
\hline
\end{tabular}

Tab. 35: Koeffizienten des statistischen Modells mit den Einflussvariablen Anteil der Flachwasserbereiche (AntFWB), dem Hydrophytendeckungsgrad (KO_Hydr) und dem logarithmierten Wert der Konnektivität (In_con), $\mathrm{R}^{2}=0,66$

Aus den modellierten Phytoplankton-Biomassen und den hydrologischen Bedingungen können folgende Parameter, für die ökologische Funktionsfähigkeit und die Wasserqualität der bearbeiteten Gewässerabschnitte abgeleitet werden.

Fläche der bei mittlerer Wasserführung angebundenen Abschnitte (ha)

Der Indikator zeigt das Ausmaß an dynamischen Wasserflächen. Je höher die Werte, desto höher wurden Gewässerabschnitte im Sinne der ökologischen Funktionsfähigkeit von Augewässern bewertet, da die aufgebaute Biomasse in die Donau exportiert werden kann und dem Nahrungsnetz des Fluss/Au-System zur Verfügung steht.

Flächenanteile mit Chl-a $>15 \mu \mathrm{g} \mathrm{l-1}$ und $<30 \mu \mathrm{g} \mathrm{l-1}$

Anteile der Gewässerfläche mit einem modellierten mittleren Chl-a-Gehalt zwischen 15 und $30 \mu \mathrm{g}$ 1-1 in Prozent. Zeigt die Fläche hochproduktiver, im Sinne der Wirkung auf die Donau günstigen Bereiche an. Bei Chl-a-Gehalten > $30 \mu \mathrm{g} 1-1$ sind negative Auswirkungen auf das Gewässer, wie zu hohe Sauerstoffzehrung, möglich.

Chl-a-Gehalt in nicht durchströmten Gewässern (Abweichung vom Schwellenwert $10 \mu \mathrm{g} 1-1$ in Prozent) 
Hinsichtlich des Leitbildes sind mesotrophe Verhältnisse in isolierten Bereichen des Ausystems zu empfehlen. Um dies abzubilden, wurden die wenig angebundenen Gewässerabschnitte mit einer mittleren Geschwindigkeit kleiner $0,05 \mathrm{~m} \mathrm{~s}-1$ (wurde als nicht durchströmt angenommen) selektiert und der mittlere Chl-a-Gehalt mit dem Schwellenwert $10 \mu \mathrm{g} \mathrm{1-1,} \mathrm{der} \mathrm{als} \mathrm{Richtwert} \mathrm{für} \mathrm{mesotrophe}$ Verhältnisse angenommen wurde, verglichen. Höhere Werte wurden auf Grund der zunehmenden Eutrophierung als negativ bewertet, niedrigere Werte wegen der, verglichen mit dem Richtwert, geringeren biologischen Aktivität. $10 \mu \mathrm{g}$ 1-1 entspricht dabei $100 \%$ (Abb. 88).

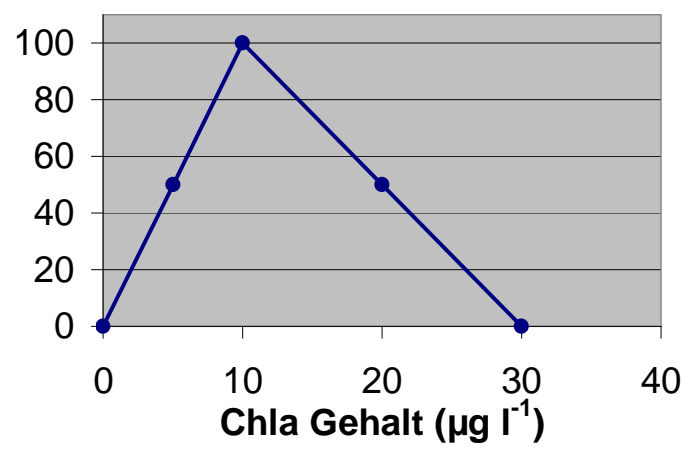

Abb. 88: Berechnung der Abweichung von einem Chl-a-Schwellenwert von $10 \mu \mathrm{l}$-1 in wenig durchströmten Gewässerabschnitten in \%.

Modelladaptierung:

Im Rahmen der Bearbeitungen wurden die entwickelten Modelle auf den erhöhten Nutzerdruck adaptiert. Dabei lassen sich zwei Hauptpunkte darstellen. Erstens ist durch die steigende Besucherfrequenz auch eine erhöhte Badenutzung von Teilbereichen der Lobau zu erwarten. Dies hat auch eine Auswirkung auf die Makrophytenverteilung (siehe oben). Durch die Modell-Verschränkung geht die Veränderung in der Abundanz der Wasserpflanzen auch in die Phytoplankton-Modellierung ein.

Zweitens bietet das Modell die Möglichkeit, den direkten Einfluss von Nährstoffeintrag (Phosphat als limitierender Nährstofffaktor für die Primärproduktion) in das Modell einzubauen. In wenig von der Donau beeinflussten Bereichen und isolierten Nebengewässern kann der Phosphateintrag durch Badenutzung einen wesentlichen Anteil im Phosphorhaushalt darstellen (Tab. 36). In einer vereinfachenden und für die Modellierung anwendbare Art und Weise wurde dabei der mittlere Phosphat-Gehalt im Gewässer (Mittelwert PO4, 1995 - 2010) auf die Gewässerfläche hochgerechnet und dem Eintrag durch Badegäste 1t. Schulz (1981) gegenübergestellt.

Die Anzahl der Badegäste wurde aus den im Wahlmodell berechneten Nutzerfrequenzen der umliegenden Wege berechnet (ArcGIS, Spitzentage der Szenarien), mit der Annahme das $25 \%$ der Wegnutzer das Gewässer zum Baden nutzen.

$\mathrm{Da}$ in den Badegewässern sehr geringe Phosphatwerte vorliegen, wurde angenommen, dass eingetragenes Phosphat ohne wesentliche Verluste direkt in Phytoplankton-Biomasse eingebaut wird. Die berechnete Erhöhung des Phosphatpools bewirkt einen dreimal so hohen Anstieg der Phytoplankton-Biomasse (nach Jensen et al. 1994, Verhältnis C:Chl-a wie 30:1 und Redfield ratio: C:P wie 106:1). 


\begin{tabular}{|lll|}
\hline $\begin{array}{l}\text { Dechantlacke } \\
\text { Fläche: }\end{array}$ & 39730 & $\mathrm{~m}^{2}$ \\
mittlere Tiefe: & 2,87 & $\mathrm{~m}$ \\
mittlerer P-Gehalt: & 2 & $\mu \mathrm{g} \mathrm{l-1}$ \\
\hline Phosphatpool: & 228,05 & $\mathrm{~g}$ in Dechantlacke \\
& & \\
PO4-Eintrag: & 0,094 & $\mathrm{~g}$ pro Gast und Tag \\
\hline bei 300 Badenden: & 28 & Eintrag g pro Tag \\
entspricht & 12 & \% Erhöhung des Phosphatpools \\
entspricht & 37 & \% Erhöhung der Phytoplankton-Biomasse \\
\hline bei 900 Badenden: & 85 & Eintrag g pro Tag \\
entspricht & 37 & \% Erhöhung des Phosphatpools \\
entspricht & 111 & \% Erhöhung der Phytoplankton-Biomasse \\
\hline
\end{tabular}

\begin{tabular}{|c|c|c|}
\hline \multicolumn{3}{|l|}{ Panozzalacke } \\
\hline Fläche: & 41920 & $\mathrm{~m}^{2}$ \\
\hline mittlere Tiefe: & 1,4 & $\mathrm{~m}$ \\
\hline mittlerer P-Gehalt: & 2 & $\mu \mathrm{g} 1-1$ \\
\hline Phosphatpool: & 117,38 & $\mathrm{~g}$ in Dechantlacke \\
\hline PO4-Eintrag: & 0,094 & g pro Gast und Tag \\
\hline bei 150 Badenden: & 14 & Eintrag g pro Tag \\
\hline entspricht & 12 & \% Erhöhung des Phosphatpools \\
\hline entspricht & 36 & $\%$ Erhöhung der Phytoplankton-Biomasse \\
\hline bei 450 Badenden: & 42 & Eintrag g pro Tag \\
\hline entspricht & 36 & \% Erhöhung des Phosphatpools \\
\hline entspricht & 108 & $\%$ Erhöhung der Phytoplankton-Biomasse \\
\hline
\end{tabular}

\begin{tabular}{|lll|}
\hline Donau-Oder-Kanal & & \\
Fläche: & 122460 & $\mathrm{~m}^{2}$ \\
mittlere Tiefe: & 2 & $\mathrm{~m}$ \\
mittlerer P-Gehalt: & 1 & $\mu \mathrm{g} \mathrm{l-1}$ \\
\hline Phosphatpool: & 244,92 & $\mathrm{~g}$ in Dechantlacke \\
& & \\
PO4-Eintrag: & 0,094 & $\mathrm{~g}$ pro Gast und Tag \\
\hline bei 100 Badenden: & 9 & Eintrag g pro Tag \\
entspricht & 4 & \% Erhöhung des Phosphatpools \\
entspricht & 12 & \% Erhöhung der Phytoplankton-Biomasse \\
\hline bei 300 Badenden: & 28 & $\begin{array}{l}\text { Eintrag g pro Tag } \\
\text { entspricht }\end{array}$ \\
entspricht & 12 & \% Erhöhung des Phosphatpools \\
\hline
\end{tabular}

Tab. 36: Nährstoffeintrag durch Badenutzung am Beispiel der drei Hauptbadegewässer der Unteren Lobau, Dechantlacke, Panozzalacke und Donau-Oder-Kanal. Eintrag pro Badegast 0,094 g pro Tag (Schulz 1981). Die Anzahl der Badegäste wurde aus den Nutzerfrequenzen an Spitzentagen der Szenarien 1999 und 2025 abgeleitet.

\subsubsection{Ergebnisse:}

\subsection{Makrophyten}

Die Menge der submersen Makrophyten (Hydrophyten) ist neben der mittleren Strömungsgeschwindigkeit bei höheren Donauwasserführungen $\left(\mathrm{Q} 4000 \mathrm{~m}^{3} \mathrm{~s}-1\right)$ von der Geomorphologie der Gewässerabschnitte abhängig. Während in tieferen Abschnitten $(>2 \mathrm{~m})$ das Vorkommen von Hydrophyten, wegen des Konkurrenznachteiles um Licht gegenüber Phytoplankton, 
eingedämmt ist (Großenzersdorfer Arm, Donau-Oder-Kanal, Schönauer Wasser), ergeben sich in den flachen Gewässerabschnitten sehr hohe Makrophyten-Deckungsgrade.

Die Auswirkungen einer erhöhten Besucherfrequenz (Szenario A - 2025) können lokal sehr groß sein (siehe Sensitivitätskarten, Kap. 6.2.3), bezogen auf Gewässerabschnitte sind sie jedoch sehr gering und im Vergleich mit 1999 nicht darstellbar.

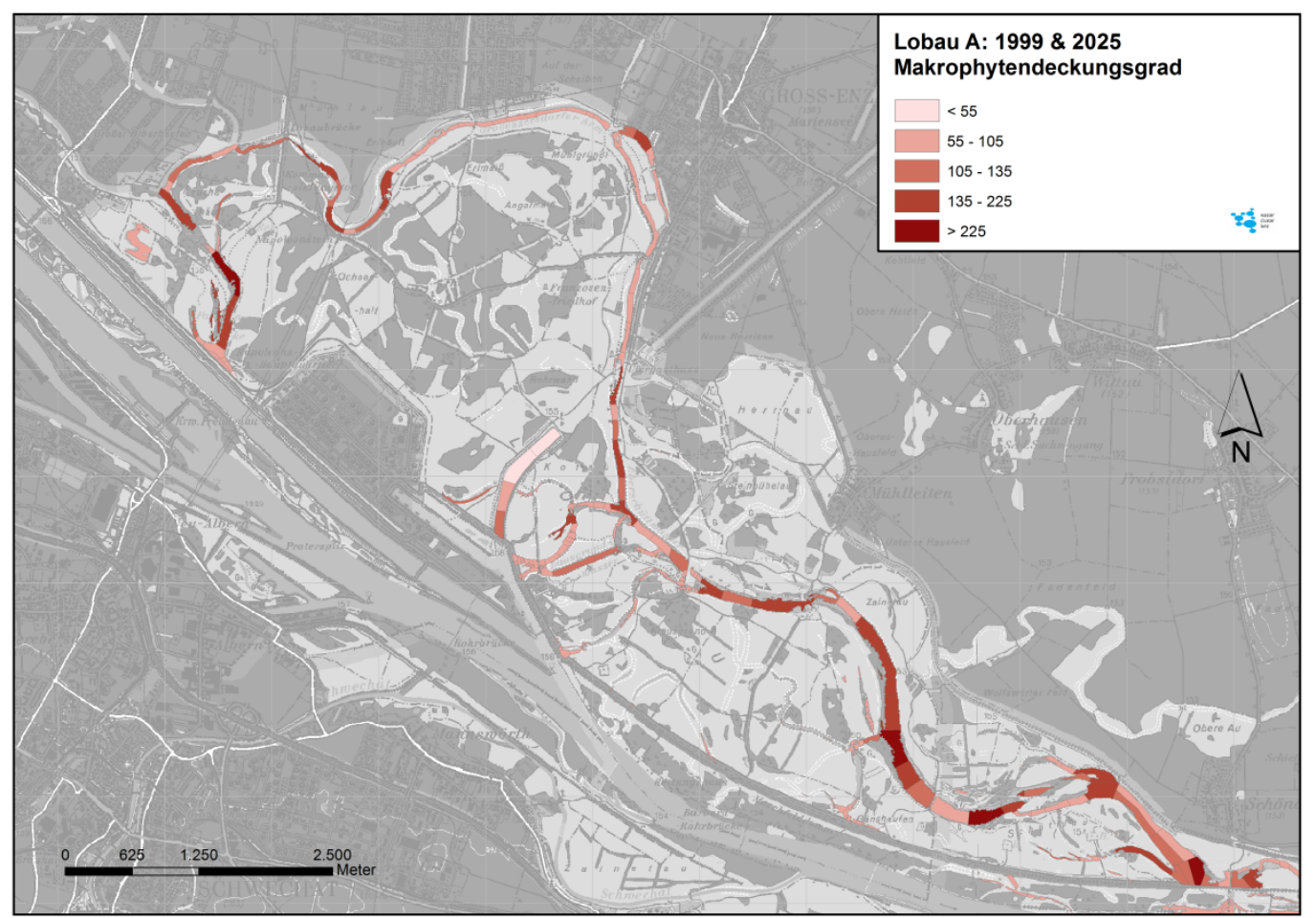

Abb. 89: Modellierte Werte der Makrophytendeckung (aufsummierte, umgerechnete Kohler-Schätzungen) für das Gewässerszenario A mit den Besucherfrequenzen von 1999 und 2025 (Baart, 2008 adaptiert).

Bei Gewässerszenario B zeigen sich die Auswirkungen der Anbindung (durchströmend) und der damit einhergehenden erhöhten Fließgeschwindigkeit. Hydrophyten erreichen im Hauptgewässerzug sehr geringe Deckungsgrade und sind auf die flacheren Bereiche mit verminderter Strömung beschränkt. 


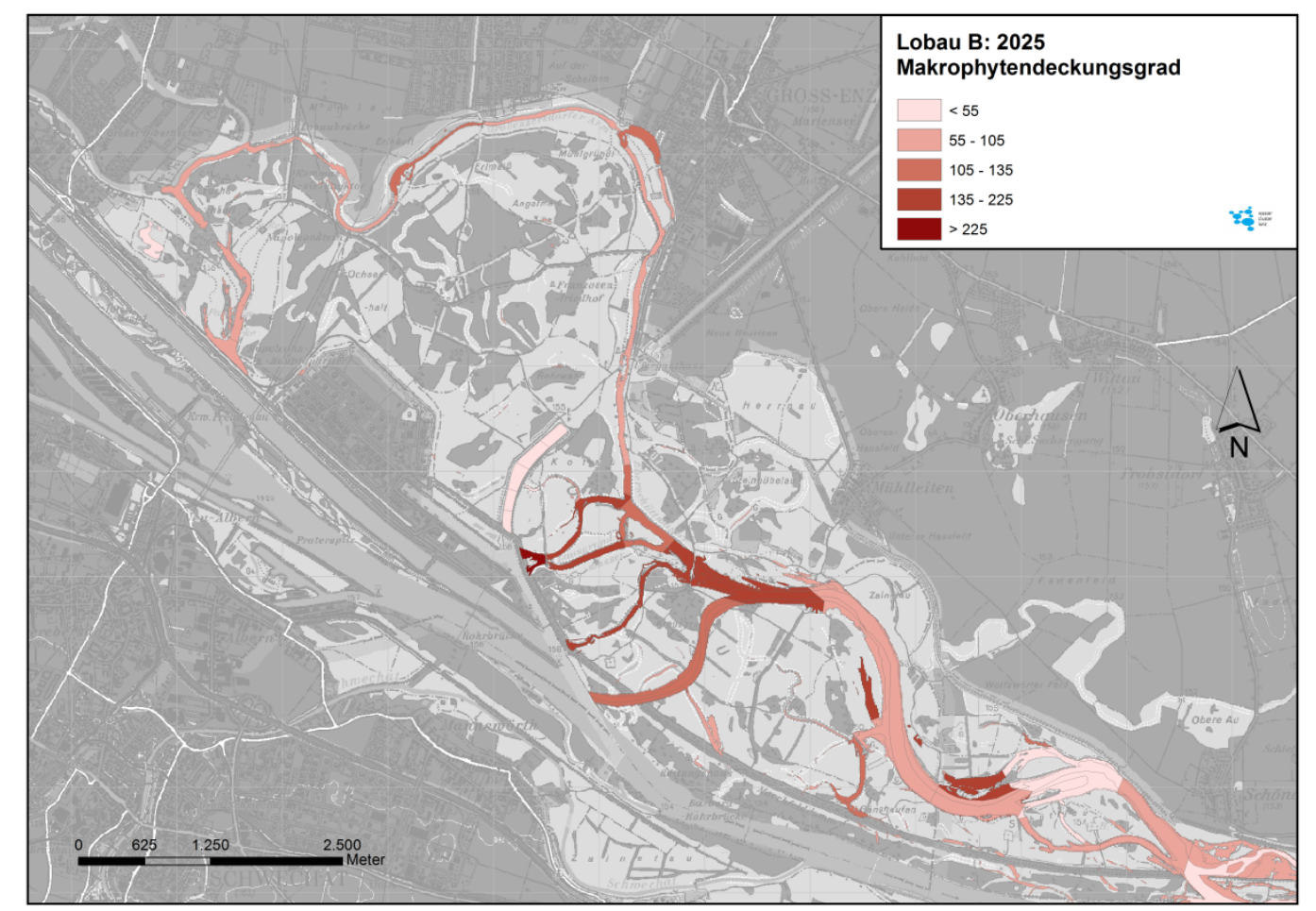

Abb. 90: Modellierte Werte der Makrophytendeckung (aufsummierte, umgerechnete Kohler-Schätzungen) für das Gewässerszenario B mit den Besucherfrequenzen von 2025 (Baart, 2008 adaptiert).

\subsection{Phytoplankton-Biomasse}

Als Indikator für die Phytoplankton-Biomasse wurde der mittlere jährliche Chlorophyll-a-Gehalt in den Gewässern modelliert (Abb. 91 bis Abb. 93). Dabei können 3 Gruppen unterschieden werden, die Gewässer der Oberen Lobau, der Hauptgewässerzug in der Unteren Lobau und die Nebengewässer der Unteren Lobau.

Während in der Oberen Lobau durchwegs sehr geringe mittlere Chl-a-Werte vorliegen $(<6,5 \mu \mathrm{g} 1-1)$, kommt es im Hauptgewässerzug zu einer starken Differenzierung (6,5 - $25 \mu \mathrm{g} 1-1)$. Der frühere Seitenarm der Donau ist an seinem unteren Ende mit dem Hauptfluss verbunden, ab einer mittleren Wasserführung kommt es zu einem Wassereintrag aus der Donau (rückströmend). Da dies auch einen Eintrag von Nährstoffen bedeutet, wird bei einer Anbindung die Phytoplankton-Primärproduktion stimuliert. Je näher am Einrinn (Schönauer Schlitz) also ein Gewässerabschnitt liegt, desto höher ist sein Anbindungsgrad (=Konnektivität, d a-1) und desto höher ist auch sein mittlerer Chl-a-Gehalt. Ausnahmen bestehen in sehr flachen Abschnitten wie dem unteren Teil des Schönauer Wassers. Ebenfalls sehr unterschiedlich ist der Chl-a-Gehalt in den Nebengewässern, die keine (oder sehr geringe) Oberflächenwasseranbindung mit der Donau aufweisen. Die Höhe des mittleren Chl-aGehaltes zeigt eine Abhängigkeit von der Lage im Augebiet und der Geomorphologie der Gewässer. Während flache, in bewaldeten Gebieten liegende Nebengewässer sehr geringe Werte aufweisen, zeigen die größeren, nahe des Marchfeld-Schutzdammes gelegenen Nebengewässern sehr hohe Chl-aWerte (bis zu 35 g 1-1). Zum Beispiel zählen die Badegewässer Panozzalacke und Dechantlacke zu dieser Gruppe. 


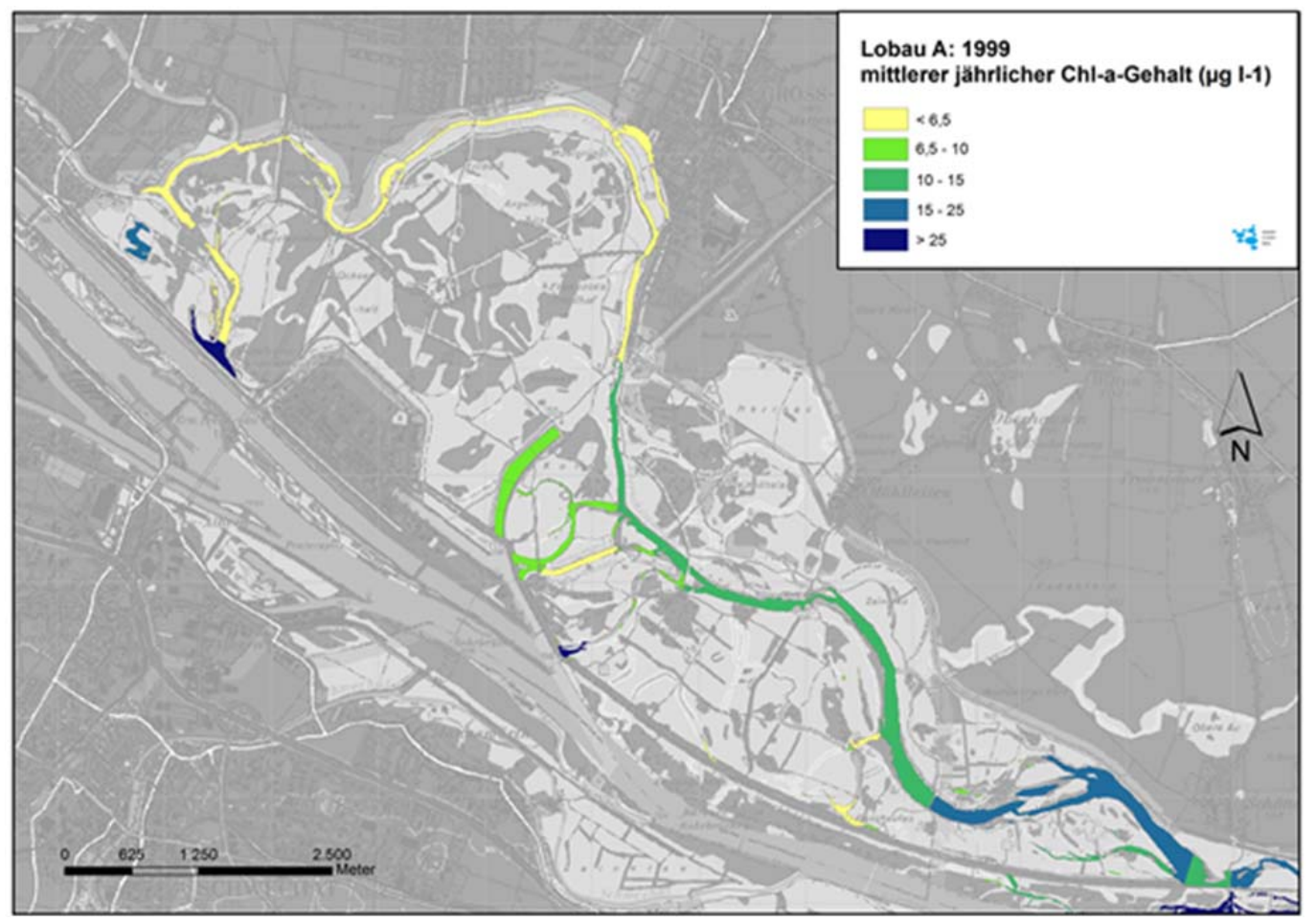

Abb. 91: Modellierte Werte des mittleren jährlichen Chlorophyll-a-Gehaltes in der Unteren Lobau - Gewässerszenario A - 1999.

Modelliert man den Chlorophyll-a-Gehalt mit erhöhtem Besucherdruck (Gewässerszenario A - 2025) fallen zwei wesentliche Punkte auf. Im Hauptgewässerzug kommt es nur zu geringfügigen Änderungen der Chl-a-Gehalte, da erstens die Frequenz der Besucher in den unteren Bereichen der Lobau weniger stark ansteigt (siehe auch Modellierung der Besucherfrequenz, Kap. 4), und zweitens die Auswirkung durch eine häufigere Anbindung wesentlich abgeschwächt wird. In den Bereichen nahe der Eingänge in die Lobau (Saltenstraße, Groß-Enzersdorf), sowie in den Badegewässern zeigt sich jedoch ein Anstieg des Chl-a-Gehaltes.

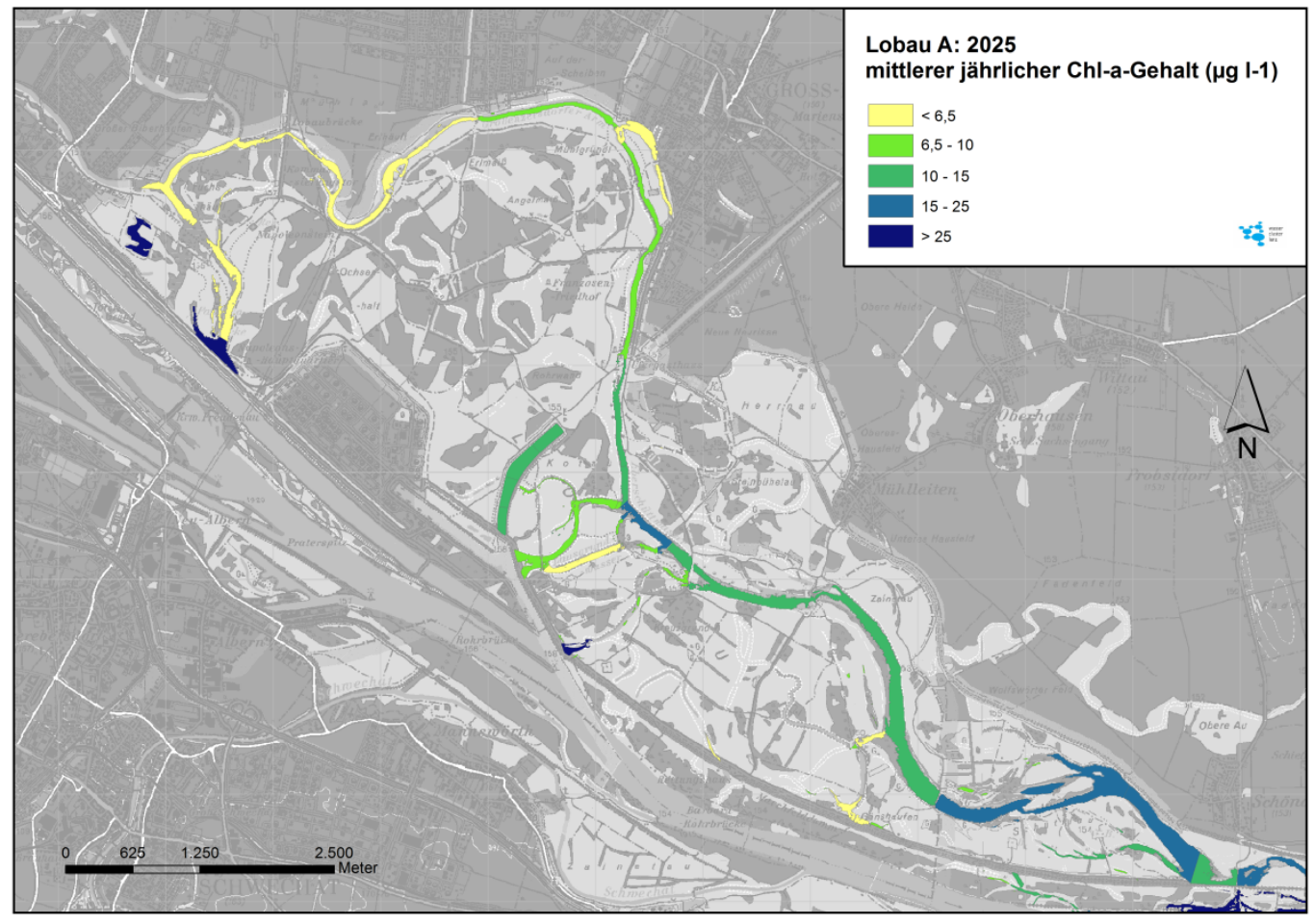

Abb. 92: Modellierte Werte des mittleren jährlichen Chlorophyll-a-Gehaltes in der Unteren Lobau - Gewässerszenario A - 2025. 
Gewässerszenarios B - 2025 repräsentiert eine vollkommen geänderte hydrologische Situation in den Gewässern der Lobau. Durch die direkte Anbindung an die Donau unterhalb des Ölhafens wird auch der Hauptgewässerzug in Strömungsrichtung der Donau durchströmt, was eine Dynamisierung und einen erhöhten Nährstoffeintrag sowie eine hohe mittlere Chl-a-Konzentrationen (15 - $30 \mu \mathrm{g}$ l-1) zur Folge hat. Auch in kleineren Nebengewässern am Hauptgewässerzug ist dies erkennbar.

Durch Rückströmung ab mittleren Wasserführungen der Donau bis weit in die Obere Lobau hinein, kommt es auch im Groß-Enzersdorfer-Arm zu stark erhöhten Chl-a-Gehalten. Die Auswirkungen der höheren Besucherfrequenz 2025 werden dadurch überlagert.

Die Badegewässer Dechantlacke, Panozzalacke und Donau-Oder-Kanal sind von der geänderten Wasserführung nicht betroffen.

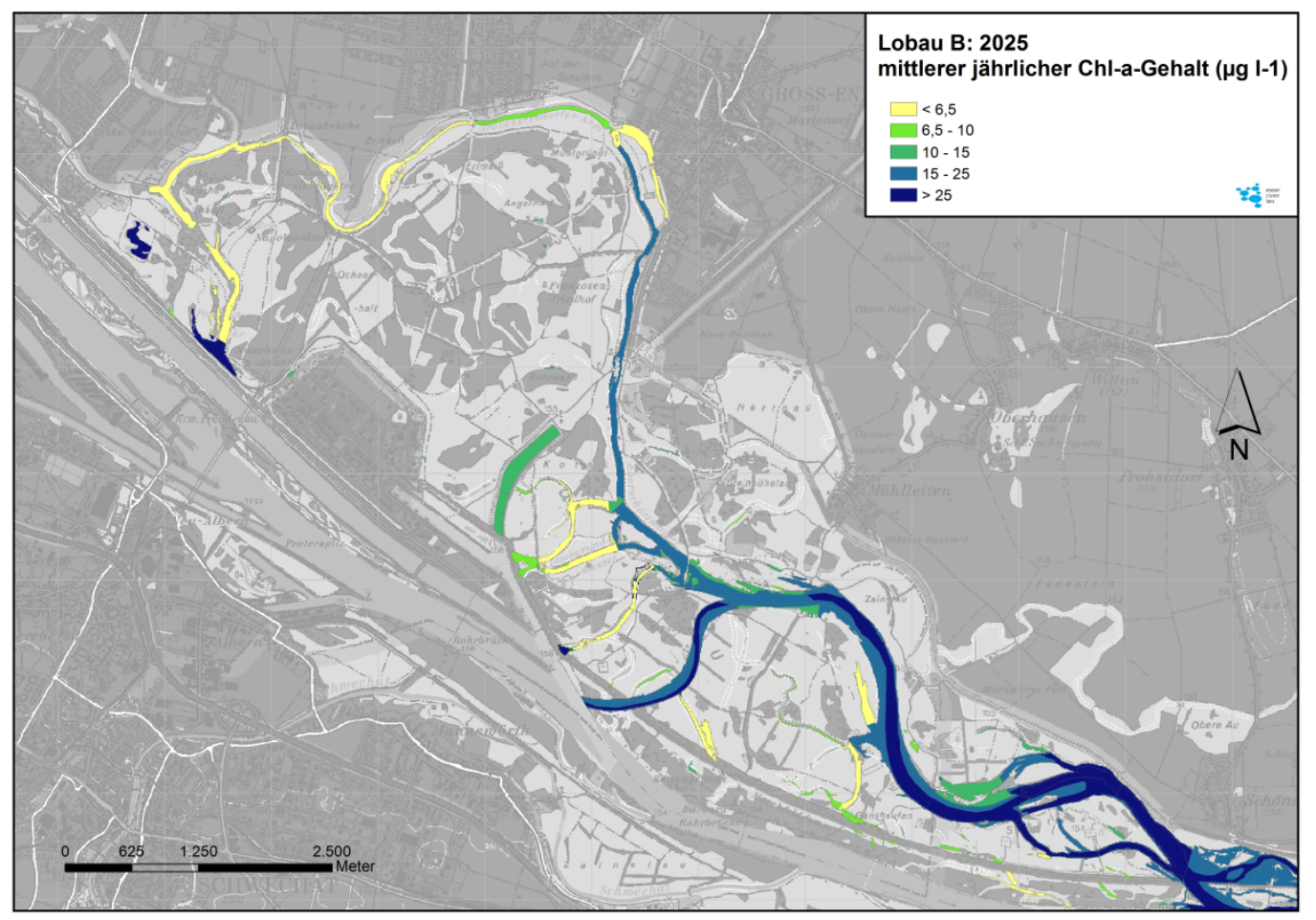

Abb. 93: Modellierte Werte des mittleren jährlichen Chlorophyll-a-Gehaltes in der Unteren Lobau - Gewässerszenario B - 2025.

\subsubsection{Bewertung des Szenarios 2025}

\subsubsection{Sensitivität:}

Vergleicht man die Modellergebnisse für Szenario A mit Besucherfrequenzen von 1999 und 2025 (Makrophyten- und Chl-a-Modellierung), zeigen sich die Bereiche, in denen die stärksten Auswirkungen eines erhöhten Besucherdrucks zu erwarten sind (Abb. 94). Diese sind eindeutig in den Badegewässern Panozzalacke und Dechantlacke, sowie in den direkt in der Nähe der Eingänge zur Lobau zu erwarten. 


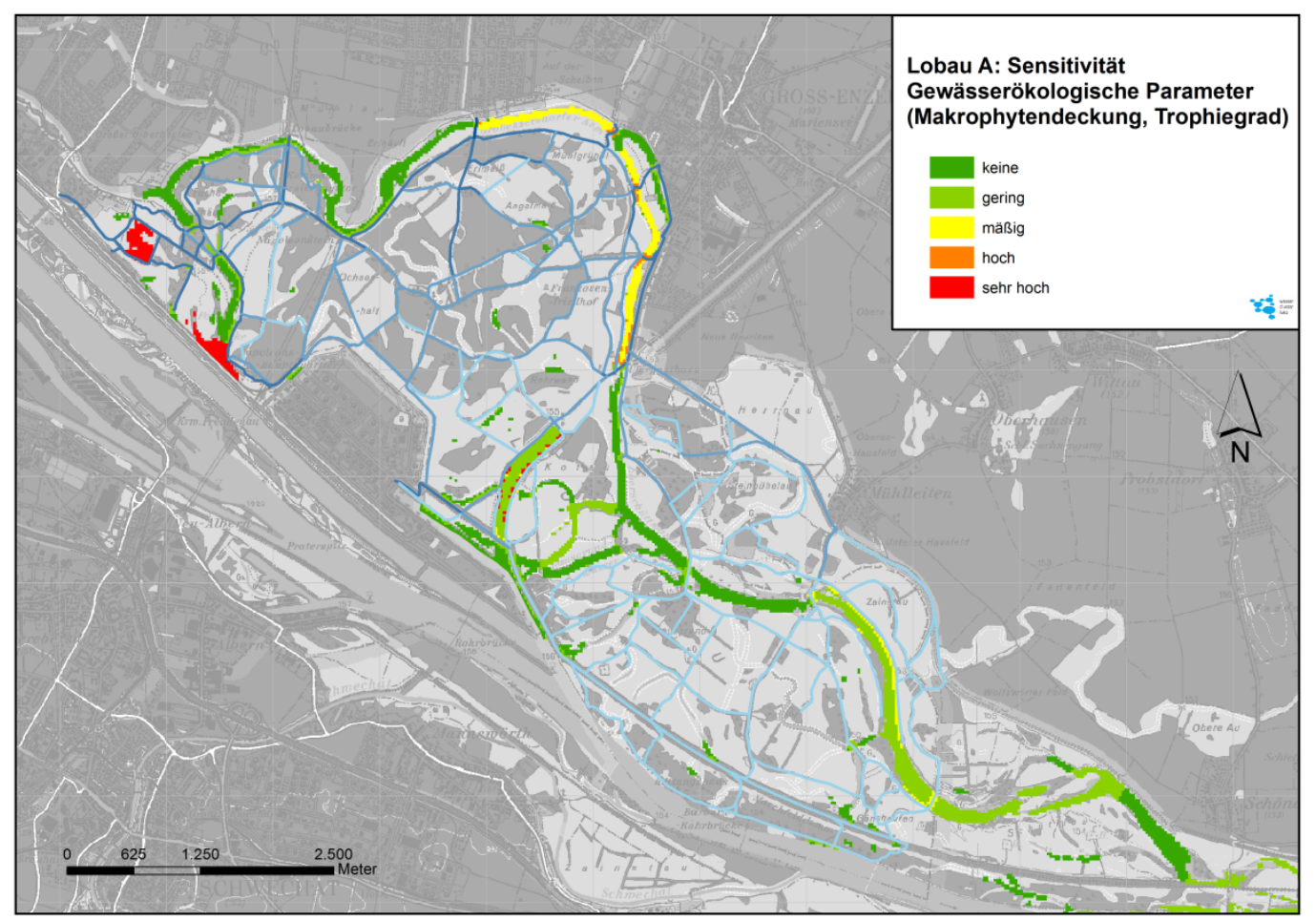

Abb. 94: Sensitivitätskarte Gewässerökologie. Hinterlegt mit den Besucherfrequenzen 2025.

Bei den derzeitigen Besucherfrequenzen ist die Badeeignung an Panozzalacke und Dechantlacke während eines durchschnittlichen Sommers durchgehend gewährleistet (MA 39, Labors für Umweltmedizin). Allerdings kommt es durch die hohe Biomasse zu stark Sauerstoff zehrenden Bedingungen während der Nachtstunden, was zu nahezu sauerstofffreien Tiefenschichten und hygienisch bedenklichen Bedingungen bei längeren Schönwetterperioden führt.

Eine Erhöhung des Besucherdruckes (Besucherfrequenzen 2025) hat eine potentielle Verdoppelung mit negativen Auswirkungen auf die Wasserqualität zur Folge (Tab. 36).

\subsubsection{2 Ökologische Funktionsfähigkeit:}

Unter dem Begriff ökologische Funktionsfähigkeit wurden mit ArcGIS folgende, aus den Modellierungen abgeleitete Indikatoren, zusammengefasst.

- Fläche der bei mittlerer Wasserführung angebundenen Abschnitte (ha)

- Flächenanteile mit Chl-a $>15 \mu \mathrm{g} \mathrm{l-1} \mathrm{und}<30 \mu \mathrm{g}$ 1-1 im Hauptgewässerzug (ha)

- Chl-a-Gehalt in nicht durchströmten Gewässern (Abweichung vom Schwellenwert $10 \mu \mathrm{g}$ 1-1) in $\%$

Dazu wurde die relative Lage der Indikatorwerte zu den positiven Maximal- und Minimalwerten des jeweiligen Indikators festgelegt und in einen Prozentwert umgerechnet (der höchste positive Wert eines Indikators entspricht dabei $100 \%$ ). Die Prozentwerte für die einzelnen Indikatoren wurden gemittelt und in einer 5-stufigen Skala dargestellt (Abb. 95).

Als Bereiche mit (sehr) guter ökologischer Funktionsfähigkeit werden dabei die unteren Abschnitte des Hauptgewässerzuges ausgewiesen, während in den dammnahen Nebengewässern und den nicht, bzw. wenig angebundenen Bereichen des Hauptgewässerzuges und der Oberen Lobau Defizite herrschen. 


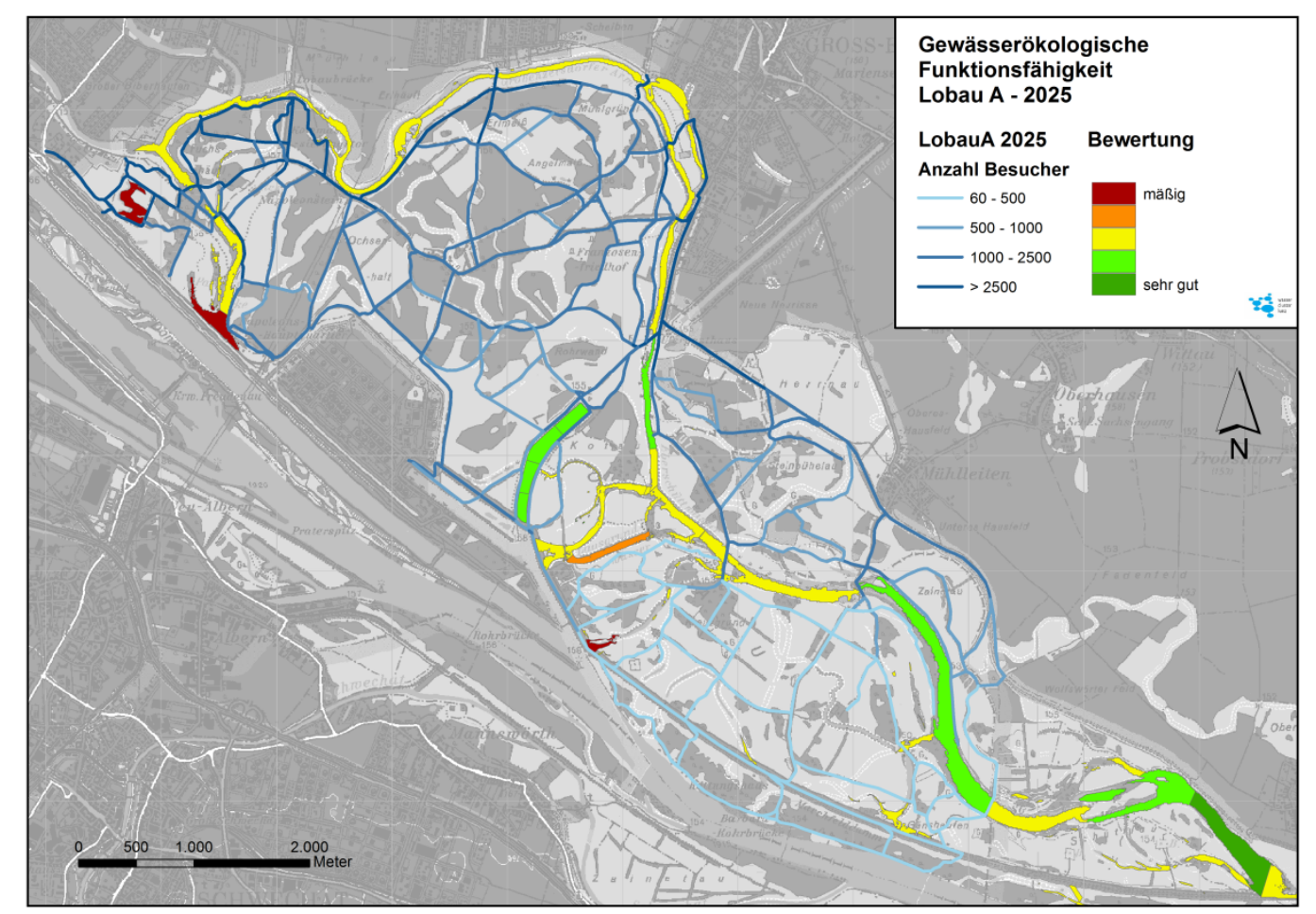

Abb. 95: Darstellung der ökologischen Funktionsfähigkeit der Gewässer für das Gewässerszenario A. Hinterlegt mit den Besucherfrequenzen 2025.

\subsubsection{Bewertung der Szenarien}

Aus gewässerökologischer Sicht ist lediglich die Bewertung des Gewässerszenarios B sinnvoll. Die Ausprägung des Lobau-Vorlandes hat zwar Auswirkungen auf die Modellergebnisse von Makrophyten und Chl-a-Gehalt, diese sind jedoch sehr gering. Auch die daraus berechneten Indikatorwerte zeigen wesentlich größere Unterschiede zwischen den gewässerspezifischen Ausprägungsformen, als zwischen den verschiedenen Vorlandgestaltungen (Tab. 37).

\begin{tabular}{|c|c|c|c|c|c|c|}
\hline & $\begin{array}{c}\text { Lobau A } \\
1999\end{array}$ & $\begin{array}{c}\text { Lobau A } \\
2025 \\
\end{array}$ & $\begin{array}{c}\text { Lobau A, } \\
\text { Vorland A }\end{array}$ & $\begin{array}{c}\text { Lobau A, } \\
\text { Vorland B }\end{array}$ & $\begin{array}{c}\text { Lobau B, } \\
\text { Vorland A }\end{array}$ & $\begin{array}{c}\text { Lobau B, } \\
\text { Vorland B }\end{array}$ \\
\hline $\begin{array}{l}\text { Flächenanteile mit } \\
\text { Chla }>15 \text { und }<30 \\
\mu \mathrm{g} \mathrm{l} 1-1 \text { (mean) (ha) }\end{array}$ & 13,2 & 13,1 & 13,1 & 13,2 & 36,9 & 37,2 \\
\hline $\begin{array}{l}\text { Fläche der bei MW } \\
\text { angebundenen } \\
\text { Abschnitte (+OL } \\
\left.\text { bei } 10 \mathrm{~m}^{3} \mathrm{~s}-1\right)(\mathrm{ha})\end{array}$ & 54,4 & 54,4 & 54,4 & 54,4 & 158,1 & 158,1 \\
\hline $\begin{array}{l}\text { Abweichung von } \\
\text { Chla- } \\
\text { Schwellenwert } \\
(10 \mu \mathrm{g} 1-1) \text { in } \\
\text { wenig } \\
\text { durchströmten } \\
\text { Gewässern (\%) }\end{array}$ & 52,2 & 57,3 & 57,1 & 56,7 & 40,8 & 39,6 \\
\hline
\end{tabular}

Tab. 37: Zusammenstellung der berechneten Indikatorwerte für die ökologische Funktionsfähigkeit.

\subsubsection{Gewässerszenario B - Sensitivität}

Die Sensitivitätskarte zeigt wie schon bei Gewässerszenario A die Badegewässer Dechantlacke und Panozzalacke als Bereiche, wo die größten Auswirkungen eines erhöhten Besucherdruckes zu erwarten sind (Abb. 96Abb. 96). Der Rückstrom in den Groß-Enzersdorfer-Arm und der damit verbundene Nährstoffeintrag vermindert die Auswirkungen erhöhter Besucherfrequenz. 


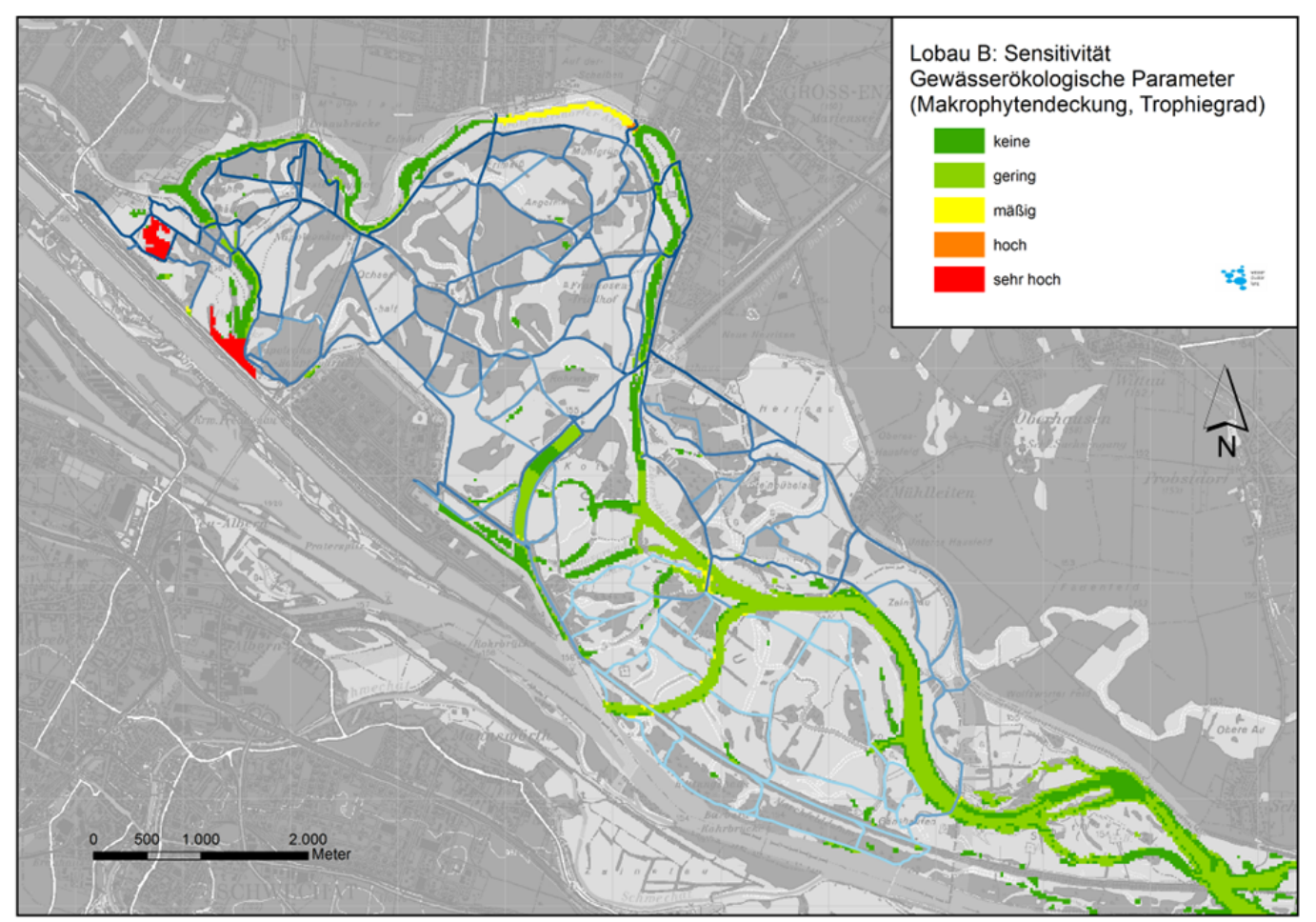

Abb. 96: Sensitivitätskarte Gewässerökologie für das Gewässerszenario B. Hinterlegt mit den Besucherfrequenzen 2025.

\subsubsection{Gewässerszenario B - ökologische Funktionsfähigkeit}

Während durch die Vorlandgestaltung kaum Auswirkungen auf die Gewässerökologie feststellbar sind, bringt eine Anbindung massive Verbesserungen verglichen mit dem derzeitigen Zustand (Besucherfrequenzen 1999) mit sich (Abb. 97). Durch die Anbindung zeigt sich eine deutliche Erhöhung an auen-ökologisch wertvollen Flächen. Der gesamte Hauptgewässerzug bis zum GroßEnzersdorfer -Arm ist wieder in das Abflussgeschehen der Donau integriert, hohe Primärproduktionsraten und direkte Interaktion mit dem Fluss sind möglich.

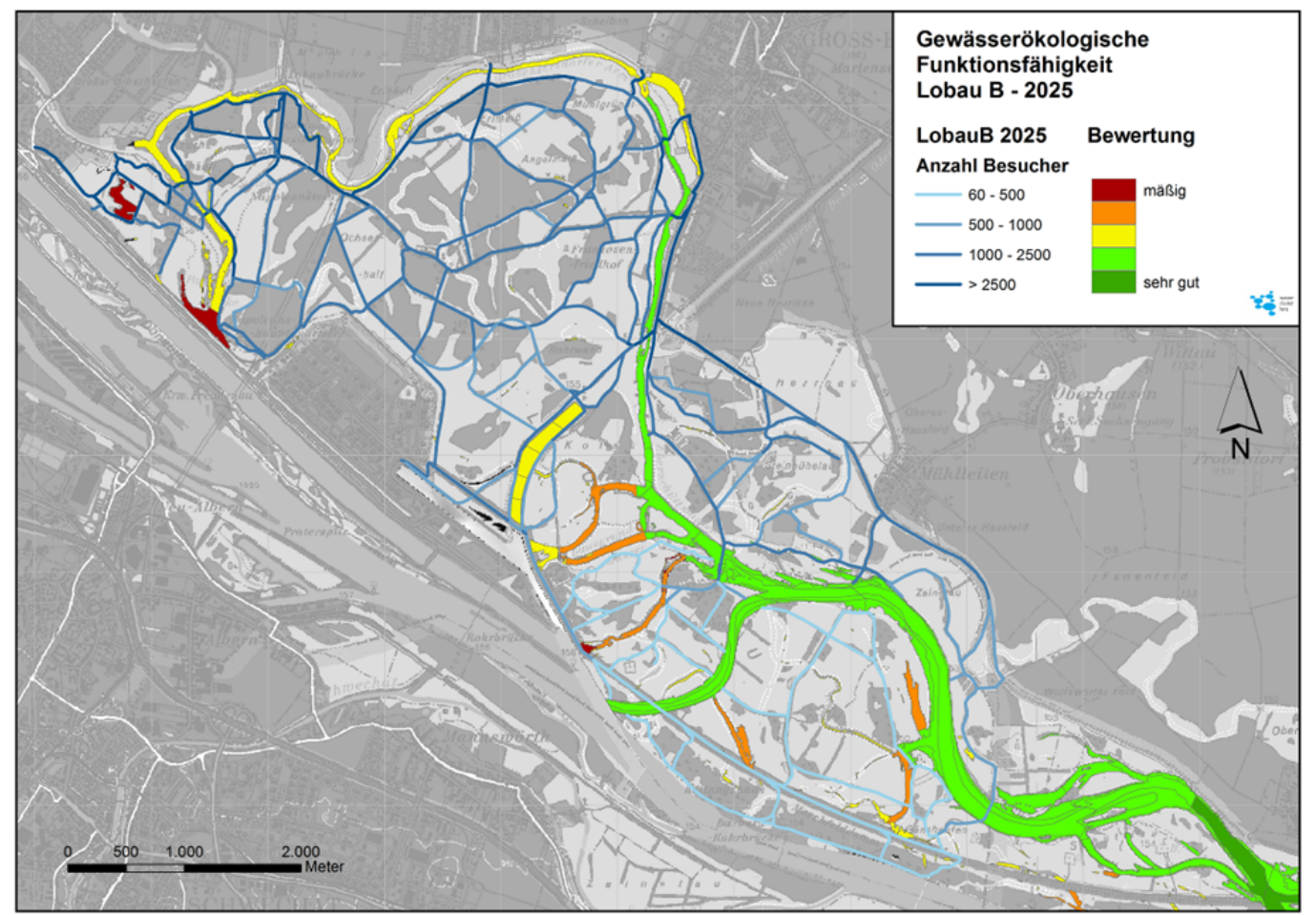

Abb. 97: Darstellung der ökologischen Funktionsfähigkeit der Gewässer für das Gewässerszenario B. Hinterlegt mit den Besucherfrequenzen 2025. 


\subsection{Bewertungskriterien aus Sicht der Wildtierökologie}

Aus der Sicht der Wildbiologie stellen Menschen im Lebensraum von Wildtieren immer einen Störfaktor dar. Es stellt sich daher prinzipiell nicht die Frage, ab welchen Besucherzahlen eine Störung der Wildtiere gegeben ist, sondern welche Bereiche der Lobau als besonders sensibel hinsichtlich Störungseinfluss einzustufen sind und wo die größten Überlappungen zwischen sensiblen Lebensräumen und (hohem) Besucheraufkommen zu identifizieren sind.

\subsubsection{Einleitung}

Als Indikatorarten für die Beurteilung der Habitateignung und in weiterer Folge der Sensibilität der unterschiedlichen Habitate in der Oberen und Unteren Lobau gegenüber Störung durch Erholungssuchende wurden Tierarten gewählt, die typisch für den Nationalpark Donauauen sind, deren Aktionsradius relativ groß ist und deren Habitatansprüche leicht zu identifizieren sind. Als typische Tierart für Auenlandschaften, deren Vorkommen an das Vorhandensein von Wasser gebunden ist, wurde der Eurasische Biber (Castor fiber L.) gewählt. Als weitere Tierarten wurden das Reh (Capreolus capreolus L.) und der Rothirsch (Cervus elaphus L.) ausgesucht. Beide kommen im Untersuchungsgebiet typischerweise vor und haben ähnliche Habitatansprüche, weshalb sie auch zusammen betrachtet werden. Für beide Gruppen (Biber und Reh-/Rotwild) wurden Beurteilungskriterien für die Lebensraumeignung bzw. die Sensibilität des Lebensraumes gegenüber Störeinflüssen innerhalb der Vegetationsperiode entwickelt.

\subsubsection{Eurasischer Biber (Castor fiber L.)}

Für den Biber ist natürlich das wesentlichste Kriterium das Vorhandensein von Wasser, wobei hier die Fließgeschwindigkeit und die Gewässertiefe von Bedeutung sind, sowie das Auftreten von häufigen und plötzlichen Wasserstandsschwankungen (z.B. Müller-Schwarze \& Sun 2003). Darüberhinaus sind Kriterien der Lebensraumeignung die Uferbeschaffenheit (abwechslungsreiche Struktur, flache und steile Bereiche, grabbares Substrat), das Angebot an Nahrung, v.a. an Winternahrung (d.h. Weichhölzer wie z.B. Espen und Weiden, nicht weiter als $10 \mathrm{~m}$ vom Ufer entfernt) und die wasserseitige Nutzung durch den Menschen, wobei hier v.a. Bootsverkehr von Bedeutung wäre (Rosenau 2003).

\subsubsection{Reh- und Rotwild (Capreolus capreolus L. und Cervus elaphus L.)}

Für beide Tierarten sind wesentliche Faktoren für die Habitateignung die Sichtigkeit (mittlere Sichtweite in $1 \mathrm{~m}$ Höhe über dem Boden) als Indikator für den Habitatfaktor "Feindschutz/Sichtschutz", die Beschirmung durch den Bestand über dem Boden als Indikator für den Habitatfaktor „Klimaschutz“ und das Vorhandensein von optisch auffälligen Randlinien wie z. B. Straßenböschungen, Bestandesränder etc. Ein wichtiges Kriterium für die Qualität des Habitats ist auch die Beunruhigung, wobei hier wieder die Entfernung von guten Einständen zu Wegen und die Art der Beunruhigung wichtig ist (Verlassen die Besucher die vorgegebenen Wege? Laufen Hunde abseits der Wege frei herum?) Daneben kann auch die Begrünung herangezogen werden, die ein Indikator für die Verfügbarkeit von Nahrung ist, wobei zwischen Sommernahrung (gesamte Begrünung) und Winternahrung (verholzte Begrünung) zu unterscheiden ist (z. B. Bassi 2003, Debeljak et al. 2001, Partl 2001, Reimoser 1986).

\subsubsection{Methodik}

Es wurden Kriterien zur Beurteilung der Lebensraumeignung entwickelt und in weiterer Folge, die Sensibilität der verschiedenen in der Lobau vorkommenden Habitattypen gegenüber Störungseinflüssen durch den Menschen abgeleitet. Aus den unterschiedlichsten aus der Literatur bekannten Kriterien zur Beurteilung der Habitatqualität (s. auch 6.3.1 Einleitung) wurden jene ausgewählt, die als Datengrundlage vorhanden waren. Von den Projektpartnern konnten folgende Daten, jeweils für das Szenario Lobau A (ohne Gewässerszenario 1b) und Lobau B (mit Gewässerszenario $1 \mathrm{~b}$ ), zur Verfügung gestellt werden:

- Wegenetz in der Lobau inkl. Wegbreite (m) und Anzahl an Besuchern pro Wegabschnitt und Tag (ILEN)

- Vegetationstypen (CVL) 
- Gewässerausdehnung und Gewässertiefe $(\mathrm{m})$ bei einer Donauwasserführung von $2.200 \mathrm{~m}^{3} / \mathrm{s}$, $3.000 \mathrm{~m}^{3} / \mathrm{s}$ und $5.000 \mathrm{~m}^{3} / \mathrm{s}$ (WasserCluster Lunz).

Die jeweils benötigten Daten wurden im ArcGIS miteinander verschnitten und über das gesamte Gebiet ein $25 \times 25$ m-Raster gelegt, anschließend wurde für jede Rasterzelle ein Sensibilitätswert zwischen 1 und 5 berechnet: $1=$ keine Sensibilität

$2=$ geringe Sensibilität

$3=$ mäßige Sensibilität

$4=$ hohe Sensibilität

$5=$ sehr hohe Sensibilität

\subsubsection{Biber (Castor fiber L.)}

Als Datengrundlage zur Bewertung des für den Biber relevanten Habitats wurden die Vegetationstypen des CVL und die Daten zur Gewässertiefe des Wassercluster Lunz bei einer Donauwasserführung von $2.200 \mathrm{~m}^{3} / \mathrm{s}$, jeweils für das Szenario Lobau A und Lobau B (Abb. 98 und Abb. 99) herangezogen.
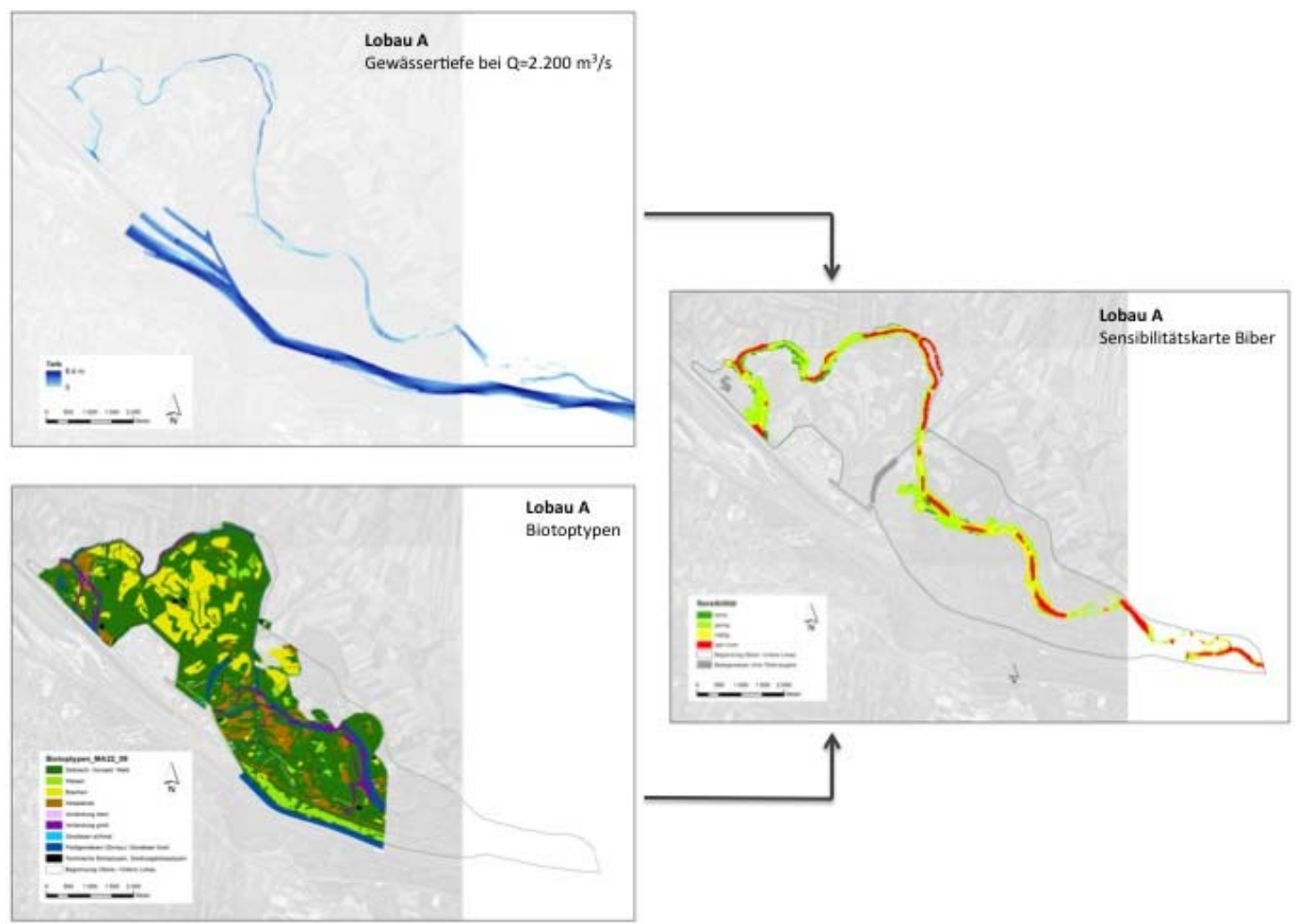

Abb. 98: Darstellung der zugrundeliegenden Daten (Gewässertiefe bei einer Donauwasserführung von $2.200 \mathrm{~m} 3 / \mathrm{s}$ und Biotoptypen) für die Erstellung einer Sensibilitätskarte für den Biber für das Szenario Lobau A (ohne Gewässerszenario 1b). 

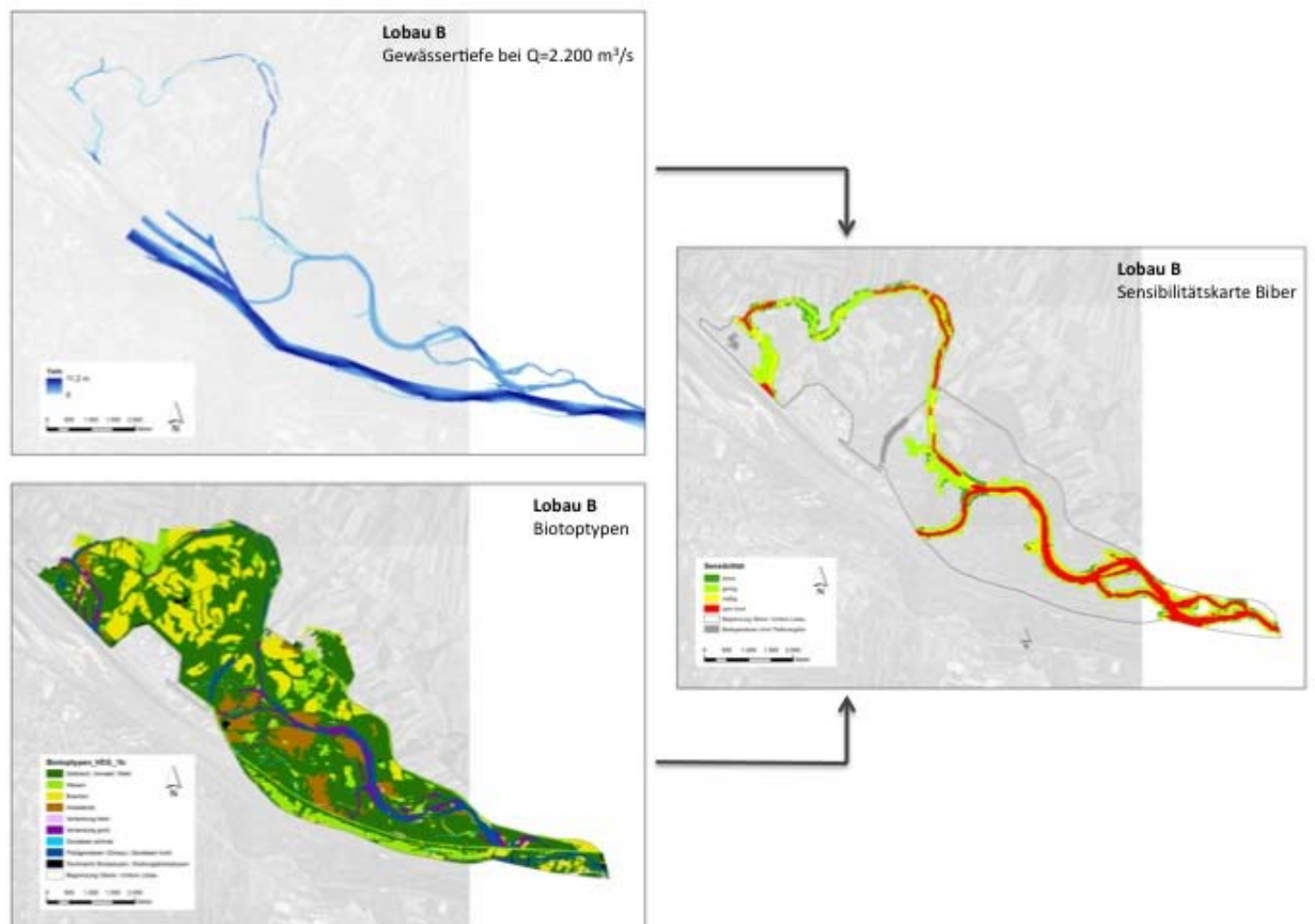

Abb. 99: Darstellung der zugrundeliegenden Daten (Gewässertiefe bei einem mittleren Donaudurchfluss von $2.200 \mathrm{~m} 3 / \mathrm{s}$ und Biotoptypen) für die Erstellung einer Sensibilitätskarte für den Biber für das Szenario Lobau B (mit Gewässerszenario 1b).

Der Biber ist an das Vorhandensein von Wasser gebunden und seine Aktivitäten beschränken sich meist auf einen Streifen entlang eines Gewässers, der nicht breiter als etwa $20 \mathrm{~m}$ ist (z.B. Schwab 2002, Maringer \& Slotta-Bachmayr 2006). Es wurde daher um alle Gewässer ein 25 m breiter Puffer gelegt und alle in diesen Pufferbereich fallenden Biotoptypen in die Sensibilitätsberechnung mit einbezogen. Die außerhalb dieses Pufferbereichs liegenden Flächen wurden nicht berücksichtigt. Trafen in einer Rasterzelle ( $25 \times 25 \mathrm{~m})$ unterschiedliche Biotoptypen zusammen, so wurde zur Bewertung der gesamten Rasterzelle der „sensibelste“ Biotoptyp herangezogen. Zur Berechnung der Sensibilität wurden innerhalb der Kriterien „Gewässertiefe“ und „Biotoptyp“ Sensibilitäts-Punkte (SP) im Wert von 0-4 für unterschiedliche Indikatoren vergeben (Tab. 38). Die Sensibilitäts-Punkte, die dabei vergeben wurden, sind umso höher, je geeigneter das dadurch beschriebene Habitat für den Biber ist. Da die Gewässertiefe ein wesentlicher Faktor für das Vorkommen des Bibers ist, wurde dieses Kriterium höher gewichtet als das Kriterium „Biotoptyp“. Nach Hartman \& Törnlöv (2006) bzw. Maringer \& Slotta-Bachmayr (2006) ist eine Gewässertiefe von 0,7-1 m bzw. 0,5 m ausreichend, um eine Besiedelung des Gewässers durch den Biber zu ermöglichen; dementsprechend wurden die SP vergeben (Tab. 38).

Biber sind dämmerungs- und nachtaktiv (z.B. Müller-Schwarze \& Sun 2003, Winter 2001). Das zeitliche Aktivitätsmuster des Bibers ist somit klar vom Hauptaktivitätszeitfenster der Erholungssuchenden getrennt, weshalb weder eine örtliche noch eine zeitliche Verlagerung der Biberaktivitäten in Folge des Besucherdrucks zu erwarten ist. Biber gelten außerdem als nicht sehr störungsanfällig und meiden die Nähe des Menschen nicht strikt (Rahm \& Baettig 1996). Die Sensibilitäts-Karte entspricht daher beim Biber einer Habitateignungs-Karte: Je sensibler ein bestimmter Bereich eingestuft wurde, desto geeigneter ist das Habitat für den Biber. 


\begin{tabular}{|l|l|c|c|c|}
\hline \multicolumn{1}{|c|}{ Kriterium } & \multicolumn{1}{|c|}{ Indikator } & SP & Gewichtung & SP ges. \\
\hline Gewässertiefe & $<0,5 \mathrm{~m}$ & 1 & 2 & 2 \\
\hline & $0,5-1,0 \mathrm{~m}$ & 2 & 2 & 4 \\
\hline & $>1,0 \mathrm{~m}$ & 4 & 2 & 8 \\
\hline Biotoptyp & Gebüsch, Vorwald, Wald & 1 & 1 & 1 \\
\hline & Wiesen & 0 & 1 & 0 \\
\hline & Brachen & 0 & 1 & 0 \\
\hline & Heißlände & 0 & 1 & 0 \\
\hline & Verlandung klein & 2 & 1 & 2 \\
\hline & Verlandung groß & 3 & 1 & 3 \\
\hline & Gewässer schmal & 4 & 1 & 4 \\
\hline & Fließgewässer, Gewässer breit & 4 & 1 & 4 \\
\hline & techn. Biotoptypen & 0 & 1 & 0 \\
\hline
\end{tabular}

Tab. 38: Schema für die Vergabe von Sensibilitäts-Punkten (0=keine / 1=gering / 2=mäßig / 3=hoch / 4=sehr hoch) für die verschiedenen Indikatoren für den Biber.

Die Summe der in einer Rasterzelle vergebenen SP wurde durch die maximal mögliche SP-Zahl dividiert und die für den entsprechenden Wert geltende Sensibilität zugeordnet (Tab. 39).

\begin{tabular}{|c|c|l|}
\hline SP-Wert & Sensibilitäts-Skala & Sensibilität \\
\hline 0 & 1 & keine \\
\hline $0,01-0,25$ & 2 & gering \\
\hline $0,26-0,50$ & 3 & mäßig \\
\hline $0,51-0,75$ & 4 & hoch \\
\hline $0,76-1,00$ & 5 & sehr hoch \\
\hline
\end{tabular}

Tab. 39: Sensibilitätsskala für den Biber.

\subsubsection{Reh- und Rotwild (Capreolus capreolus L. und Cervus elaphus L.)}

Zur Bewertung des für Reh- und Rotwild relevanten Habitats wurden als Datengrundlage wieder die Vegetationstypen des CVL herangezogen und zusätzlich Daten zu den vorhandenen Wegen in der Lobau und dem modellierten Besucheraufkommen für das Jahr 1999 („Ist-Zustand“; ILEN), jeweils für das Szenario Lobau A und Lobau B (Abb. 100 und Abb. 101). 

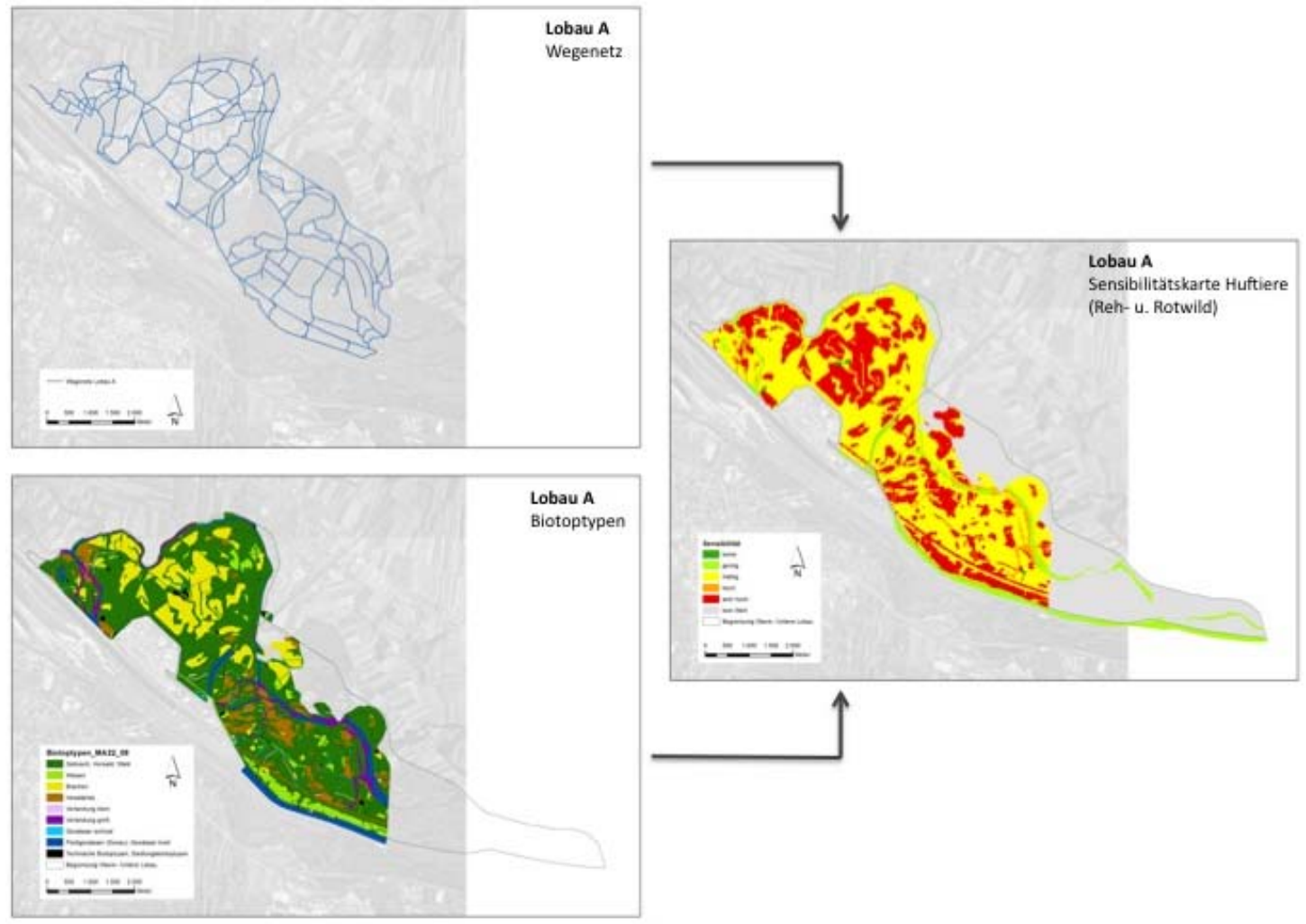

Abb. 100: Darstellung der zugrundeliegenden Daten (Wegenetz inkl. Besucherzahlen, Wegbreite etc. und Biotoptypen) für die Erstellung einer Sensibilitätskarte für Reh- und Rotwild für das Szenario Lobau A (ohne Gewässerszenario 1b).
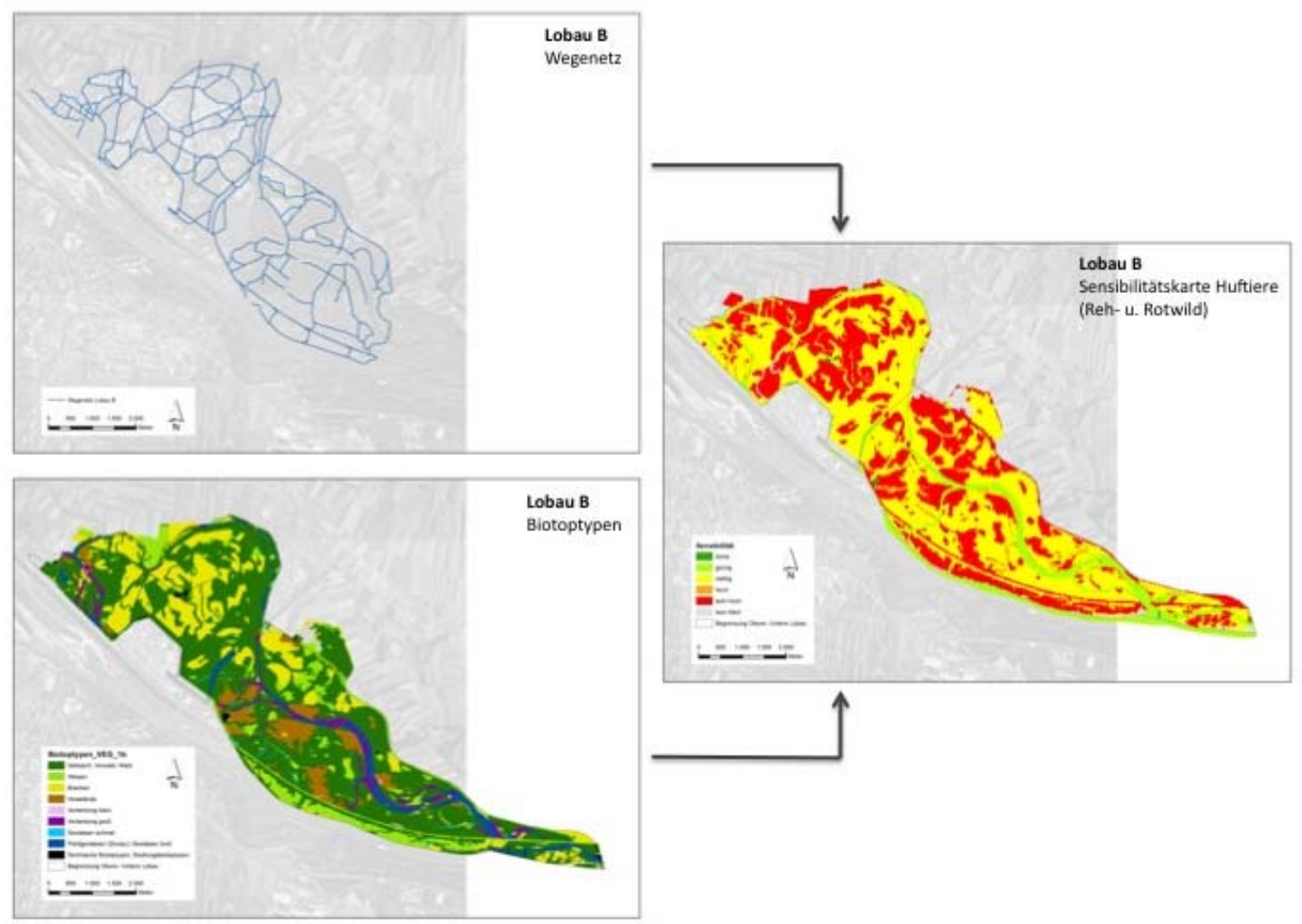

Abb. 101: Darstellung der zugrundeliegenden Daten (Wegenetz inkl. Besucherzahlen, Wegbreite etc. und Biotoptypen) für die Erstellung einer Sensibilitätskarte für Reh- und Rotwild für das Szenario Lobau B (mit Gewässerszenario 1b). 
Wie schon beim Biber wurden auch beim Reh- und Rotwild innerhalb eines Rasters von 25 x $25 \mathrm{~m}$ Sensibilitätswerte (1-5) berechnet, wobei wieder Sensibilitäts-Punkte (SP) im Wert von 0-4 vergeben wurden (Tab. 40). Die Vergabe der SP richtete sich beim Kriterium „Biotoptyp“ einerseits danach, wie wahrscheinlich sich die Tiere in diesem Biotoptyp aufhalten und andererseits danach, wie sensibel sie innerhalb dieses Biotoptyps auf Störungseinflüsse reagieren. Fluchttiere reagieren im offeneren Gelände prinzipiell sensibler auf Störung als z.B. im Wald, wo sie leichter und rascher Deckung finden können. Für die Indikatoren „Wiese“ oder „Heißlände“ wurden daher mehr SP vergeben als für den Indikator „Gebüsch, Vorwald, Wald“. Trafen in einer Rasterzelle unterschiedliche Biotoptypen zusammen, so wurde zur Bewertung jeweils der „sensibelste“ Biotoptyp herangezogen.

Weiters wurden für die Sensibilitätsberechnung noch die Kriterien „Weg“, „Wegbreite“ und „Anzahl Besucher" miteinbezogen. Tiere reagieren im Allgemeinen umso sensibler auf Störung, je unvorhergesehener sie ist. Ist in einer Rasterzelle kein Weg vorhanden, so ist sie als sensibler einzustufen, als eine Rasterzelle, wo ein Weg vorhanden ist, da im ersten Fall Besucher abseits der Wege eine ungleich größere Störung darstellen als Besucher am Weg. Ähnliches gilt auch für die Wegbreite und die Anzahl an Besuchern pro Wegabschnitt: Je schmaler der Weg und je weniger Besucher, umso sensibler ist die jeweilige Rasterzelle einzustufen. Dies ergibt sich daraus, dass Wege mit hohen Besucherfrequenzen von Rot- und Rehwild prinzipiell gemieden werden. Sie sind daher als weniger sensibel einzustufen, weil sich dort gar keine Tiere mehr aufhalten!

Daten zum Wegenetz und Besucheraufkommen waren gegenüber den Daten zur Vegetation bzw. dem Biotoptyp überrepräsentiert, was durch unterschiedliche Gewichtung der Kriterien aufgewogen wurde (Tab. 40).

\begin{tabular}{|l|l|c|c|c|}
\hline \multicolumn{1}{|c|}{ Kriterium } & \multicolumn{1}{|c|}{ Indikator } & SP & Gewichtung & SP ges. \\
\hline Weg & Weg vorhanden & 1 & 3 & 3 \\
\hline & kein Weg & 4 & 3 & 12 \\
\hline Anzahl Besucher/Wegabschn./Tag & einzelne & 4 & 1 & 4 \\
\hline & wenige & 3 & 1 & 3 \\
\hline & viele & 2 & 1 & 2 \\
\hline & sehr viele & 1 & 1 & 1 \\
\hline Wegbreite & $0,8 \mathrm{~m}$ & 4 & 1 & 4 \\
\hline & $2,0 \mathrm{~m}$ & 3 & 1 & 3 \\
\hline & $3,0 \mathrm{~m}$ & 2 & 1 & 2 \\
\hline Biotoptyp & $4,0 \mathrm{~m}$ & 1 & 1 & 1 \\
\hline & Gebüsch, Vorwald, Wald & 1 & 4 & 1 \\
\hline & Wiesen & 4 & 4 & 16 \\
\hline & Brachen & 4 & 4 & 16 \\
\hline & Heißlände & 4 & 4 & 16 \\
\hline & Verlandung klein & 1 & 4 & 4 \\
\hline & Verlandung groß & 2 & 4 & 8 \\
\hline & Gewässer schmal & 0 & 4 & 0 \\
\hline & Fließgewässer, Gewässer breit & 0 & 4 & 0 \\
\hline & techn. Biotoptypen & 0 & 4 & 0 \\
\hline
\end{tabular}

Tab. 40: Schema für die Vergabe von Sensibilitäts-Punkten (0=keine / 1=gering / 2=mäßig / 3=hoch / 4=sehr hoch) für die verschiedenen Indikatoren für Reh- und Rotwild. 
Die Summe der in einer Rasterzelle vergebenen SP wurde durch die maximal mögliche SP-Zahl dividiert und die für den entsprechenden Wert geltende Sensibilität zugeordnet (Tab. 41).

\begin{tabular}{|c|c|l|}
\hline SP-Wert & Sensibilitäts-Skala & Sensibilität \\
\hline 0 & 1 & keine \\
\hline $0,01-0,30$ & 2 & gering \\
\hline $0,31-0,60$ & 3 & mäßig \\
\hline $0,61-0,80$ & 4 & hoch \\
\hline $0,81-1,00$ & 5 & sehr hoch \\
\hline
\end{tabular}

Tab. 41: Sensibilitätsskala für Reh- und Rotwild.

\subsubsection{Ergebnisse}

Wie schon im vorherigen Kapitel „6.3.2 Methodik“ erläutert wurde, ist bei den Ergebnissen zu beachten, dass die Sensibilitäts-Karten jeweils für den Biber und für das Reh- und Rotwild unterschiedlich zu interpretieren sind. Beim Reh-/Rotwild stellt die Sensibilitätskarte tatsächlich eine Karte dar, in welcher unterschiedlich gefährdete Bereiche ausgewiesen werden: Grün sind demnach Bereiche, in denen es voraussichtlich zu keiner Störung durch Erholungssuchende kommt und rot sind die Bereiche, wo das Störpotenzial durch Besucher besonders hoch ist. Beim Biber hingegen stellt die Sensibilitätskarte eine Habitateignungskarte dar: Je sensibler ein bestimmter Bereich ist, desto geeigneter ist das Habitat für den Biber. Im Kapitel „7.4. Diskussion“ werden diese Unterschiede zwischen den beiden Tiergruppen noch einmal genauer erläutert.

\subsubsection{Eurasischer Biber (Castor fiber L.)}

Für den Biber wurden für das Szenario Lobau A (ohne Gewässerszenario 1b) insgesamt 7.130 Rasterzellen ausgewertet, was einer Fläche von 446 Hektar bzw. 4,46 km² entspricht (Abb. 103). $25 \%$ der Fläche wurden als „,sehr hoch sensibel“ eingestuft, $21 \%$ als „mäßig sensibel“, $49 \%$ als „gering sensibel“ und 5,5\% als „nicht sensibel“. Als „hoch sensibel“ wurde keine Rasterzelle eingestuft (Abb. 102).

Für das Szenario Lobau B (mit Gewässerszenario 1b) wurden insgesamt 12.458 Rasterzellen ausgewertet, was einer Fläche von 779 Hektar bzw. 7,79 km² entspricht (Abb. 104). In diesem Szenario wurden $34 \%$ der Fläche als ,,sehr hoch sensibel“ eingestuft, 12 \% als „,mäßig sensibel“, 45 \% als ,gering sensibel“ und 9\% als „nicht sensibel“. Eine Einstufung in die Kategorie „hoch sensibel“ kam wieder nicht vor (Abb. 102).

Beim Vergleich der beiden Szenarien zeigt sich, dass im Szenario Lobau B der Anteil an Flächen, die als „sehr hoch sensibel“ eingestuft wurden, um $9 \%$ höher ist als im Szenario Lobau A. Das bedeutet gleichzeitig, dass der Flächenanteil, der als Habitat für den Biber sehr gut geeignet ist, um $9 \%$ höher ist. Dieser Flächenzuwachs geht auf Kosten der als „mäßig sensibel“ eingestuften Flächen, die im Szenario Lobau B um 9\% weniger ausmachen, als im Szenario Lobau A. Der Anteil an als „nicht sensibel“ und „gering sensibel“ eingestuften Flächen bleibt in beiden Szenarien etwa gleich (Abb. 102). 


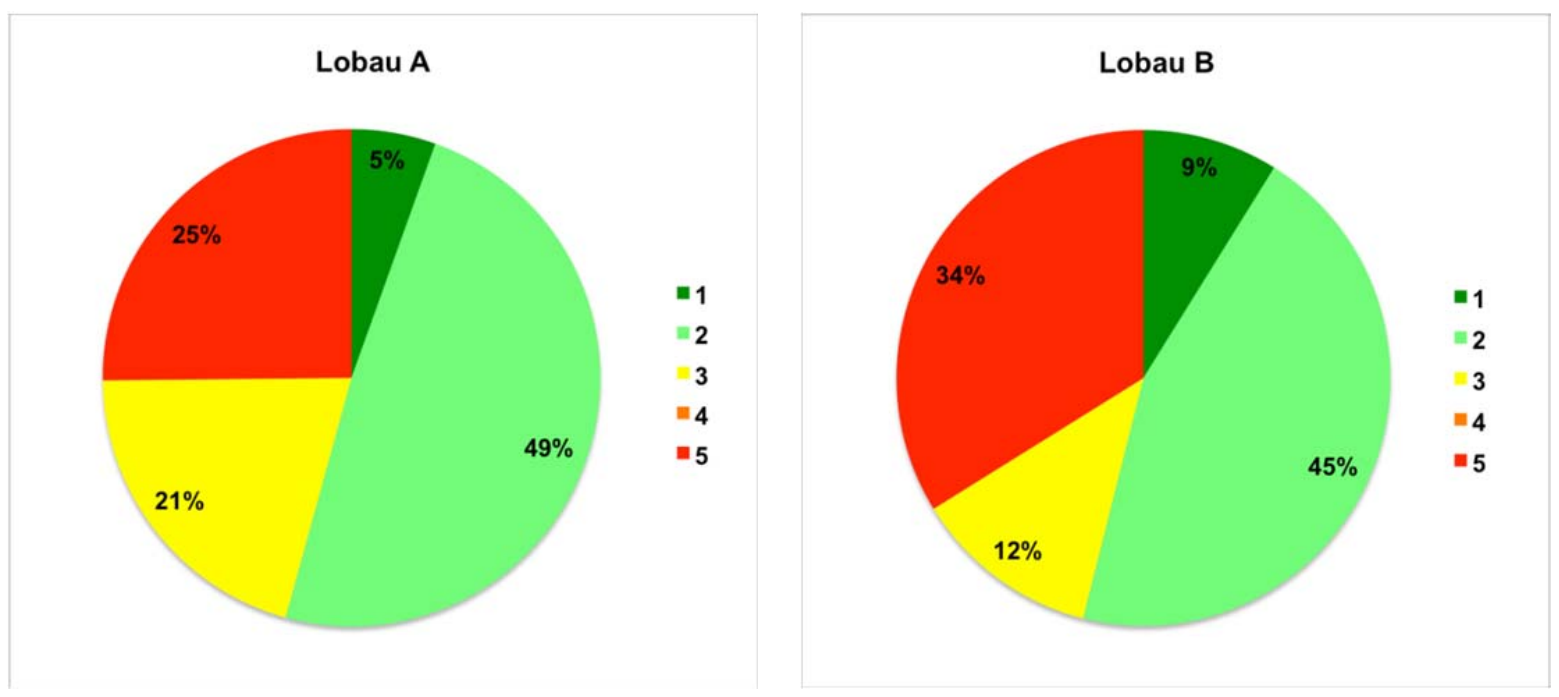

Abb. 102: Prozentueller Anteil der als unterschiedlich sensibel gegenüber Störung durch Besucher eingestuften Flächen in der Oberen und Unteren Lobau für den Biber; jeweils für das Szenario Lobau A (ohne Gewässerszenario 1b; links) und Lobau B (mit Gewässerszenario 1b; rechts). 1=nicht sensibel, 2=gering sensibel, 3=mäßig sensibel, 4=hoch sensibel, 5=sehr hoch sensibel.

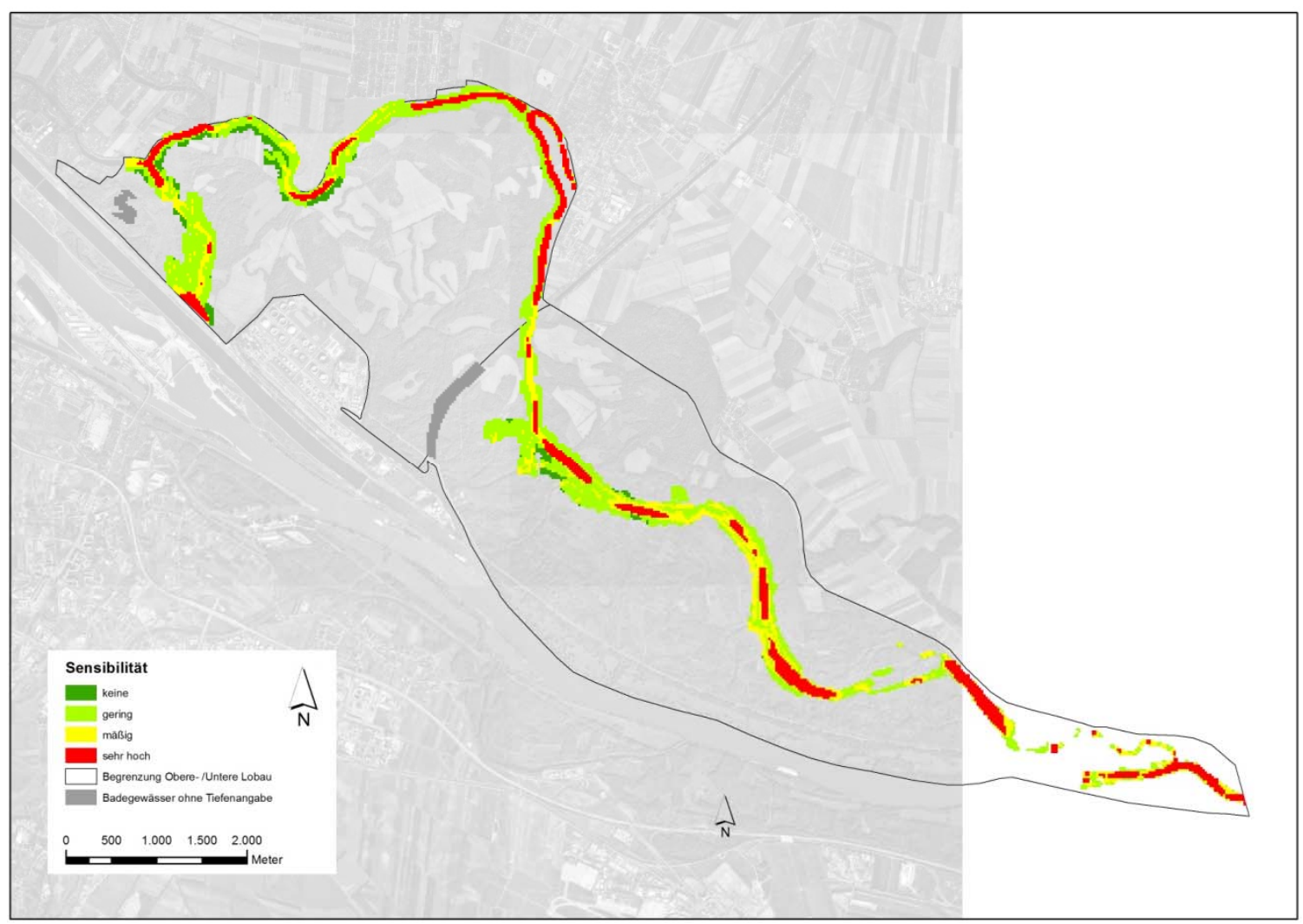

Abb. 103: Sensibilitätskarte für den Biber für das Szenario Lobau A (ohne Gewässerszenario 1b). Für die Badegewässer "Dechant Lacke“ und „Donau-Oder-Kanal“ waren keine Tiefenangaben vorhanden, weshalb für diese beiden Gewässer keine Sensibilität errechnet werden konnte. Sensibilitätsskala: keine (dunkelgrün) / gering (hellgrün) / mäßig (gelb) / hoch (orange) / sehr hoch (rot). 


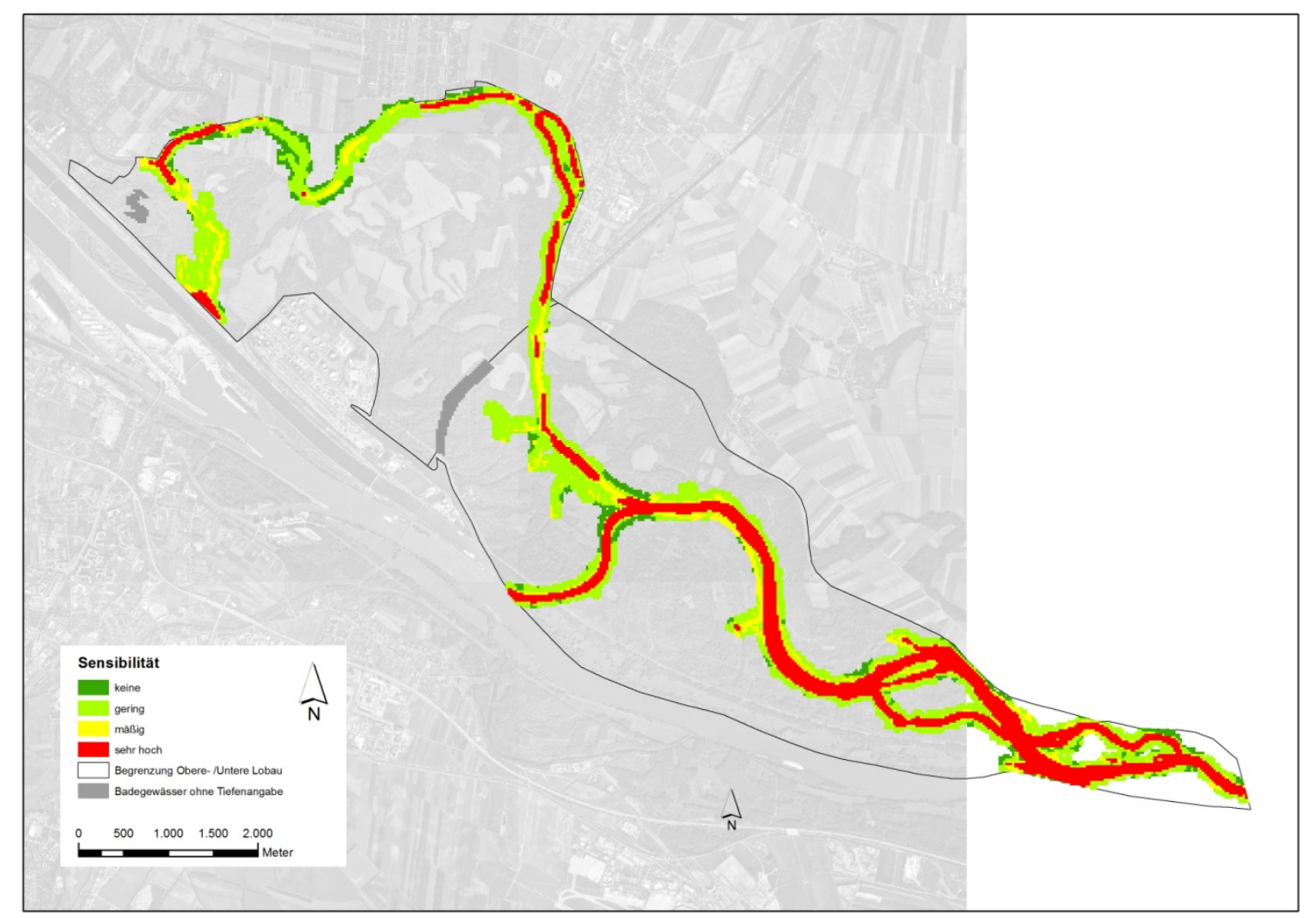

Abb. 104: Sensibilitätskarte für den Biber für das Szenario Lobau B (mit Gewässerszenario 1b). Für die Badegewässer „Dechant Lacke“ und „Donau-Oder-Kanal“ waren keine Tiefenangaben vorhanden, weshalb für diese beiden Gewässer keine Sensibilität errechnet werden konnte. Sensibilitätsskala: keine (dunkelgrün) / gering (hellgrün) / mäßig (gelb) / hoch (orange) / sehr hoch (rot).

\subsubsection{Reh- und Rotwild (Capreolus capreolus L. und Cervus elaphus L.)}

Für das Szenario Lobau A (ohne Gewässerszenario 1b) wurden für Reh- und Rotwild insgesamt 37.297 Rasterzellen ausgewertet, was einer Fläche von 2.331 ha bzw. 23,3 km² entspricht (Abb. 106). Lediglich $0,34 \%$ dieser Fläche wurde als „nicht sensibel“ gegenüber Störungseinflüssen durch Besucheraufkommen eingestuft und $11 \%$ als ,gering sensibel“. Der größte Anteil der Fläche (56\%) wurde als „mäßig sensibel“ eingestuft, 2,4\% als ,hoch sensibel“ und $30 \%$ als „,sehr hoch sensibel“ (Abb. 105).

Für das Szenario Lobau B (mit Gewässerszenario 1b) wurden insgesamt 51.155 Rasterzellen ausgewertet, was einer Fläche von 3.197 ha bzw. $32 \mathrm{~km}^{2}$ entspricht (Abb. 107). Wieder war der Anteil der als „nicht sensibel“ eingestuften Fläche mit 0,31\% am geringsten. $12 \%$ wurden als „gering sensibel“ eingestuft und mit $51 \%$ machten wieder die als „mäßig sensibel“ eingestuften Flächen den größten Anteil aus, gefolgt von den als „sehr hoch sensibel“ beurteilten Flächen (36\%). Lediglich $1 \%$ der Fläche wurde als ,hoch sensibel“ eingestuft (Abb. 105).

In beiden Szenarien ist der jeweilige Anteil der als unterschiedlich sensibel eingestuften Flächen in etwa gleich. 

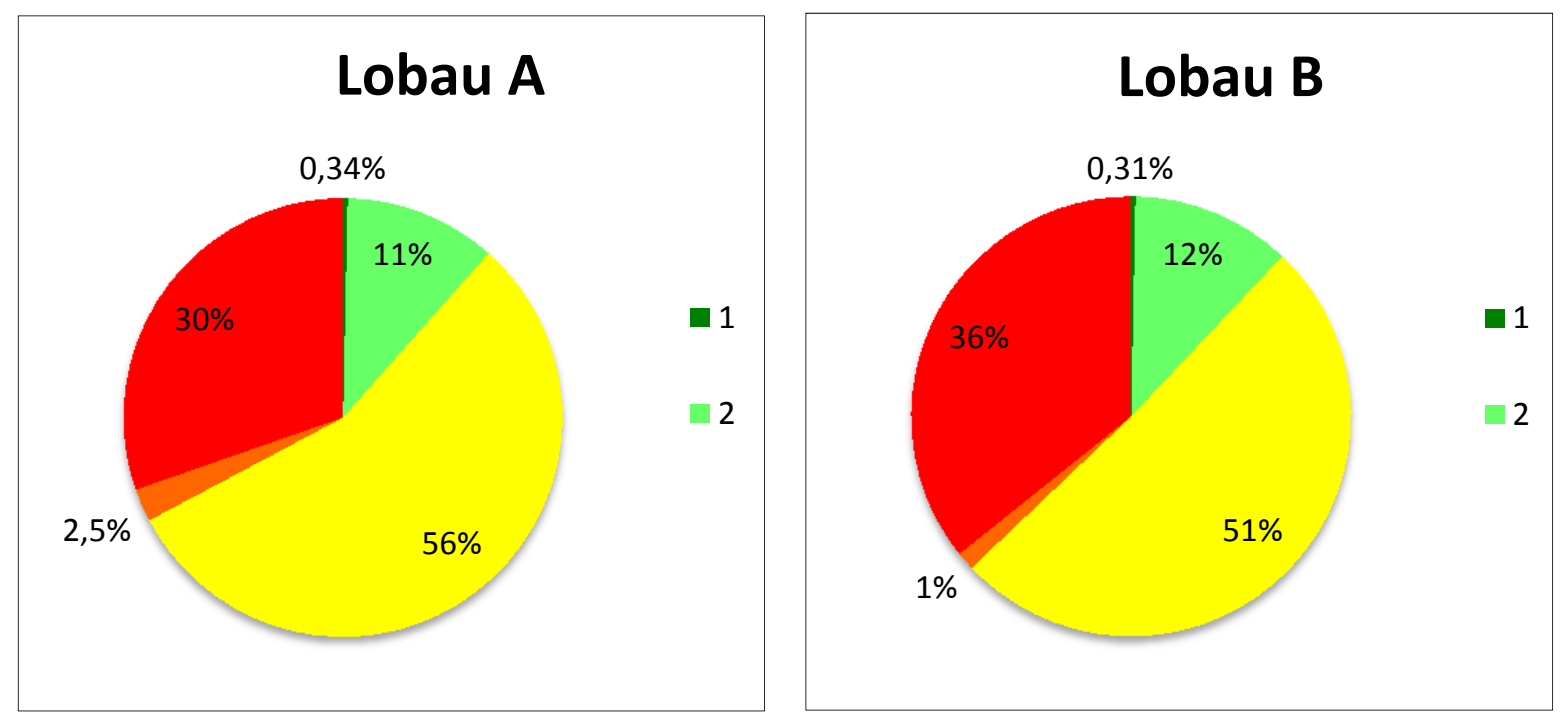

Abb. 105: Prozentueller Anteil der als unterschiedlich sensibel gegenüber Störung durch Besucher eingestuften Flächen in der Oberen und Unteren Lobau für Reh- und Rotwild; jeweils für das Szenario Lobau A (ohne Gewässerszenario 1b; links) und Lobau B (mit Gewässerszenario ab; rechts). 1=nicht sensibel, 2=gering sensibel, 3=mäßig sensibel, 4=hoch sensibel, $5=$ sehr hoch sensibel.

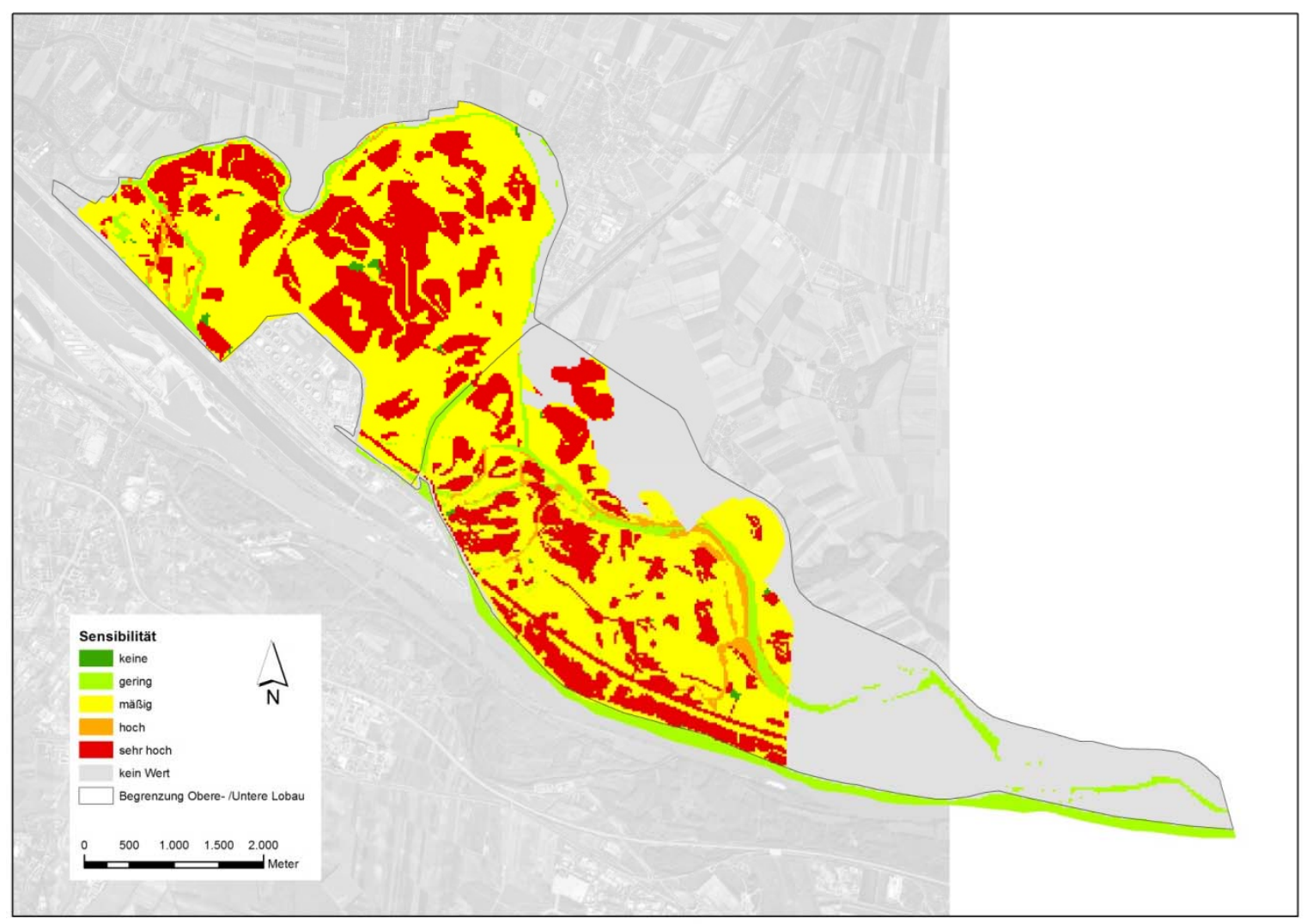

Abb. 106: Sensibilitätskarte für Reh- und Rotwild für das Szenario Lobau A (ohne Gewässerszenario 1b). Sensibilitätsskala: keine (dunkelgrün) / gering (hellgrün) / mäßig (gelb) / hoch (orange) / sehr hoch (rot). 


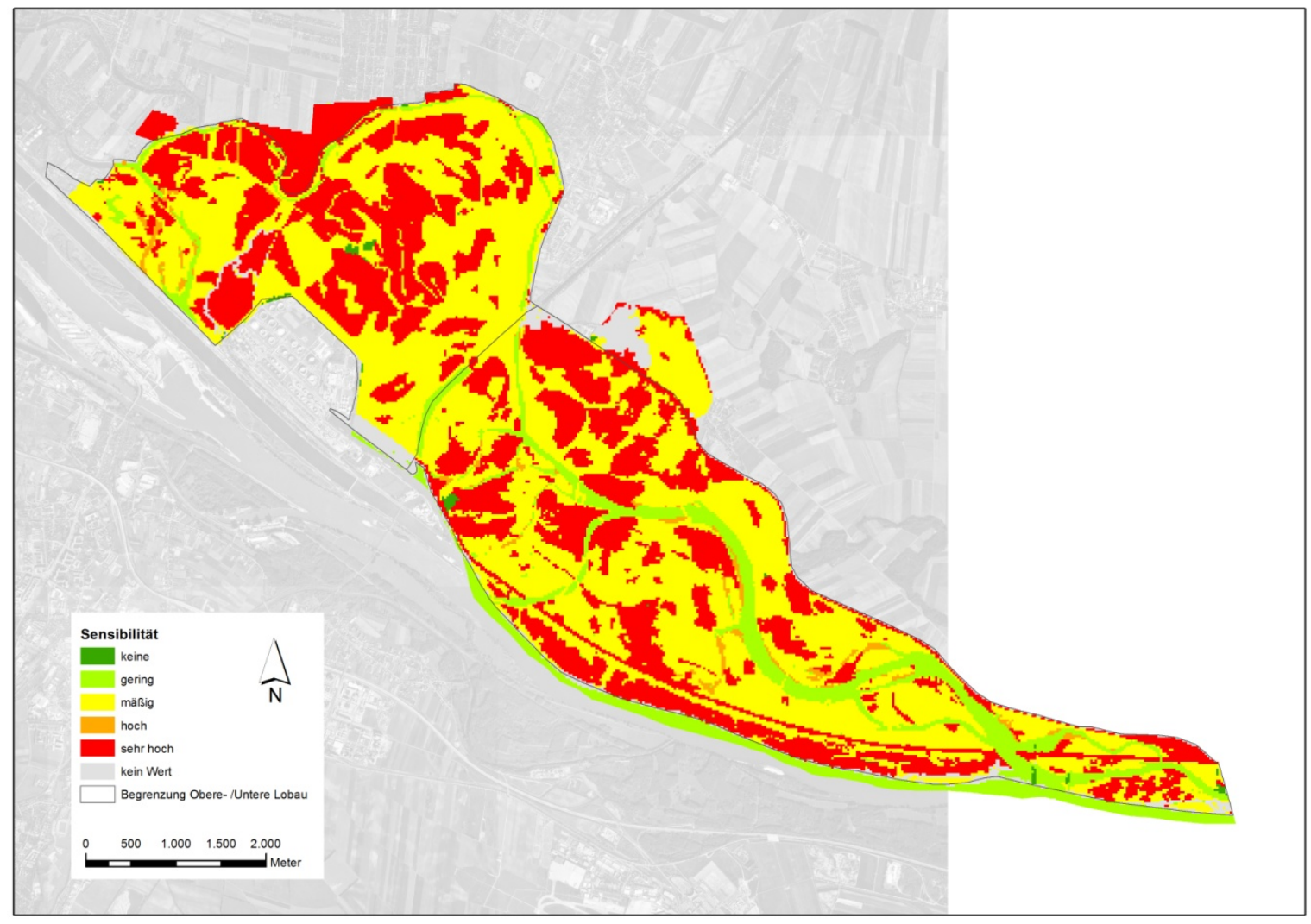

Abb. 107: Sensibilitätskarte für Reh- und Rotwild für das Szenario Lobau B (mit Gewässerszenario 1b). Sensibilitätsskala: keine (dunkelgrün) / gering (hellgrün) / mäßig (gelb) / hoch (orange) / sehr hoch (rot).

\subsubsection{Diskussion der Ergebnisse und Bewertung der Szenarien}

Die Ergebnisse und die Diskussion derselben beziehen sich alle auf die Maßnahmen innerhalb der Lobau. Die Maßnahmen im Lobau-Vorland konnten hinsichtlich ihrer Effekte auf Wildtiere zwar nicht evaluiert werden, eine vorsichtige Beurteilung und Empfehlung ist aber möglich und findet in Kapitel „,7.4 Empfehlungen aus Sicht der Wildtierökologie“" statt.

Die Ergebnisse bezüglich der Maßnahmen innerhalb der Lobau zeigen, dass sich in der Lobau der Anteil an Bereichen, die gegenüber Störung durch Erholungssuchende als mäßig bis sehr hoch sensibel eingestuft wurden, in jedem Szenario in einer Größenordnung befindet, der nicht zu vernachlässigen ist. Vor allem beim Reh- und Rotwild kann davon ausgegangen werden, dass die BesucherInnen der Lobau einen zunehmenden Störfaktor für diese darstellen. Als besonders kritisch ist eine Zunahme der Besuchsbelastung in der Unteren Lobau zu sehen, da sowohl der Biber, als auch Reh- und Rotwild innerhalb der Lobau fast ausschließlich in der Unteren Lobau vorkommen!

\subsubsection{Eurasischer Biber (Castor fiber L.)}

Die Sensibilitäts-Karte entspricht beim Biber einer Habitateignungs-Karte: Je sensibler ein bestimmter Bereich eingestuft wurde, desto geeigneter ist das Habitat für den Biber. Wie die Ergebnisse zeigen, bleibt zwar in beiden Szenarien der Anteil an gut bis sehr gut geeigneten Habitaten gleich, im Szenario „Lobau B“ ist allerdings der Anteil an sehr gut geeigneten Habitaten um einiges höher. Durch die in diesem Szenario geplante Anbindung des Hauptgewässerzuges der Unteren Lobau an die Donau, würde sich der Anteil an Gewässerflächen in der Unteren Lobau erhöhen, wodurch sich zwangsläufig auch der potenziell verfügbare Lebensraum des Bibers vergrößern würde.

Biber sind vor allem in der Dämmerung und nachts aktiv (z.B. Winter 2001, Müller-Schwarze \& Sun 2003), weshalb es wenig Überlappung zwischen den Aktivitätsphasen des Bibers und dem hauptsächlichen Besucheraufkommen gibt. Zusätzlich gelten Biber als nicht sehr störungsanfällig und meiden auch die Nähe des Menschen nicht strikt (Rahm \& Baettig 1996). Es ist daher nicht zu erwarten, dass es aufgrund des Besucherdrucks zu zeitlichen und/oder örtlichen Verlagerungen der Biberaktivitäten kommt (siehe auch Arnberger et al. 2009). Als problematisch gelten großflächige 
Beunruhigungen der Gewässer durch Bootfahren oder Wassersportarten (MUNR 1999, Rosenau 2003). Derartige Störquellen sind aber sowohl in der Unteren als auch in der Oberen Lobau zu vernachlässigen. Eine potenzielle Störquelle für den Biber sind Besucher mit Hunden, v.a. wenn diese nicht angeleint sind und frei herumlaufen. In diesem Fall wären theoretisch auch Verluste bei Jungtieren durch wildernde Hunde möglich. Aber auch hier gilt, dass es in der Dämmerung und in der Nacht wahrscheinlich nur zu einer geringfügigen Überlappung kommt. Es ist allerdings denkbar, dass es es aufgrund des höheren Besucherdrucks zu einer zeitlichen Verlagerung der Aktivitäten z.B. bei Sporttreibenden (Mountainbiker, Jogger, Nordic Walker) und Hundeausführenden in die frühen Morgen- und späten Abensdstunden kommt, wodurch sich das Risiko der Störung durch BesucherInnen erhöhen würde. Das in den Modellierungen festgestellte Ausweichverhalten der BesucherInnen von der Oberen in die Untere Lobau aufgrund des zukünftig prognostizierten höheren Besucheraufkommens in der Oberen Lobau ist prinzipiell als negativ zu werten, da sich die wertvollen Biber-Lebensräume v.a. in der Unteren Lobau befinden.

\subsubsection{Reh- und Rotwild (Capreolus capreolus L. und Cervus elaphus L.)}

Beim Reh- und Rotwild sind die Sensibilitäts-Karten anders zu interpretieren als beim Biber. Hier sind in den Karten die als unterschiedlich sensibel gegenüber Störung durch den Menschen eingestuften Bereiche innerhalb der Lobau dargestellt, was nur bedingt mit der Habitatqualität gleichzusetzen ist. Als Fluchttiere und prinzipiell tagaktive Arten reagieren beide Tierarten empfindlich auf Störung durch den Menschen. Konsequenzen aus der Anwesenheit von Menschen können sein, dass gewisse Gebiete, z.B. Wege mit hohem Besucheraufkommen gemieden werden und es zu einer Verschiebung der örtlichen und/oder zeitlichen Aktivitätsmuster kommt. Einem Wegabschnitt mit hohem Besucheraufkommen kann daher eine geringe Sensibilität bezüglich Störung zugeordnet werden, weil die Habitatqualität dementsprechend gering ist und sich dort gar keine Tiere mehr aufhalten. Umgekehrt muss aber hohe Sensibilität nicht automatisch hohe Habitateignung bedeuten. Offene Wiesenflächen sind als Habitat für Reh- und Rotwild ebenso geeignet wie geschlossene Waldflächen: Während das eine ausgezeichnete Äsungsflächen sind, sind das andere geeignete Einstandsflächen. Beide Biotoptypen stellen also Habitattypen von hoher Qualität dar, sie unterscheiden sich aber deutlich hinsichtlich ihrer Sensibilität gegenüber Störung durch Erholungssuchende. Während offene Flächen als sehr hoch sensibel einzustufen sind, sind geschlossene Waldflächen nur als gering sensibel einzustufen.

Für das Reh- und Rotwild unterscheiden sich die beiden Szenarien innerhalb der Lobau nicht wesentlich hinsichtlich des jeweiligen Anteils der als hoch und höchst sensibel eingestuften Bereiche. Bedenklich ist, dass in beiden Szenarien etwa ein Drittel der Fläche der Lobau als „hoch“ und ,sehr hoch“" sensibel eingestuft wurden. Über die Hälfte der Fläche wird als „mäßig“ sensibel eingestuft und lediglich $12 \%$ als ,nicht“ bzw. „wenig“" sensibel.

Das in jedem Szenario modellierte zukünftig höhere Besucheraufkommen wirkt sich sowohl in der Oberen als auch in der Unteren Lobau negativ aus. In der Oberen Lobau sind mit einem größeren Anteil an offenen Flächen (Wiesen, Brachen, Heißländen) mehr gegenüber Störung besonders sensible Biotoptypen zu finden. In der Unteren Lobau ist zwar der Anteil an höchst sensiblen Biotoptypen geringer, allerdings ist die Untere Lobau schon als Rückzugsgebiet für Reh- und Rotwild zu sehen. Eine Zunahme des Besucheraufkommens in diesem Bereich (auch durch das Ausweichverhalten einzelner BesucherInnen durch den höheren Besucherdruck in der Oberen Lobau) bedeutet, dass die Tiere keine Ausweichhabitate mehr finden!

Wie schon in Arnberger et al. (2009) dargelegt, entspricht die zeitliche Raumnutzung des Rot- und Rehwildes in der Lobau dem üblichen Tag-Nacht-Muster in stark durch den Menschen frequentierten Gebieten (die Tiere weichen in ihren Aktivitäten in die Nacht bzw. auf die Wochentage aus). Ein erhöhtes Besucheraufkommen unter der Woche und eine mögliche Verlagerung von sportlichen Aktivitäten in die Abend- und Morgenstunden würde sich daher besonders negativ auswirken.

Obwohl sich die beiden Szenarien „Lobau A“ und Lobau B“ hinsichtlich der Flächenanteile der als unterschiedlich sensibel eingestuften Biotoptypen nicht wesentlich unterscheiden, ist aus Sicht der Wildbiologie auch für das Reh- und Rotwild das Szenario „Lobau B“ zu empfehlen. Durch die Anbindung des Hauptgewässerzuges der Unteren Lobau an die Donau wäre das Wegenetz in der Unteren Lobau teilweise nur mehr eingeschränkt nutzbar. Der unmittelbare Lebensraum für Reh- und Rotwild würde sich zwar durch die Vergrößerung der Wasserflächen etwas verkleinern, aber der große 
Vorteil wäre eine komplette Beruhigung von Teilen der Unteren Lobau, die dadurch äußerst wertvolle Wildruhezonen darstellen würden!

\subsection{Kriterien der Erholungsplanung}

\subsubsection{Methodik und Definition der Indikatoren}

Die Bewertung der Sensibilität der Lobau aus Sicht der Erholungsplanung umfasste fünf Indikatoren (Tab. 42). Die Auswahl der Indikatoren erfolgte im Hinblick darauf, dass diese in Zusammenhang mit der Besuchermenge bzw. dem Besuchserlebnis stehen und somit die Sensibilität von Lobaubereichen gegenüber hohen Besucherströmen anzeigten. Ein weiterer Bewertungsfaktor war die Pufferwirkung des Lobau-Vorlandes. Hier stand die Beantwortung der Frage, um wie viel ein Szenario die Belastung der Lobau durch Erholungssuchende reduzieren kann, im Mittelpunkt.

Die Sensibilität der Lobau aus Sicht der Erholungsplanung wurde daher mit folgenden Indikatoren erfasst:

1. Pufferwirkung Landschaft: Die zentrale Frage war, ob eine Landschaft, im speziellen ein Landschaftselement, die Wahrnehmung von Besuchermengen beeinflusst. Dazu wurden anhand des Wahlmodells Interaktionen zwischen den dargestellten Landschaftselementen und der dargestellten Besuchermengen gerechnet. Die Wahlentscheidungen der Befragten zeigten an, dass offene Landschaften signifikant weniger präferiert wurden wenn viele Personen dargestellt waren, als halboffene oder geschlossene Landschaftselemente wie Wald. Somit werden in bestimmten Landschaftsräumen Besuchermengen als weniger hoch wahrgenommen, obwohl die gleiche Besuchermenge im Gebiet unterwegs ist.

2. Pufferwirkung Gewässer: Die Ergebnisse des Wahlmodells zeigten, dass bei größeren Gewässern bzw. dann, wenn kein Gewässer vorhanden ist, hohe Besuchermengen signifikant negativer empfunden werden.

3. Pufferwirkung Wegtypus: Die Ergebnisse des Wahlmodells zeigten, dass schmälere Wege bei hohen Besuchermengen weniger bevorzugt werden.

4. Pufferwirkung Wegbepflanzung: Die Ergebnisse des Wahlmodells zeigten, dass Landschaftselemente wie Allen und Baumreihen die Wahrnehmung von hohen Besuchermengen reduzieren können.

5. Besuchserlebnis: Dem BP Untere Lobau wurde eine höhere Sensibilität hinsichtlich der Qualität des Erholungserlebnisses zugewiesen. Aufgrund des derzeit geringeren Besuchsdrucks wird dieser Bereich als nicht überlaufen wahrgenommen. Auch vermehrte Wildtiersichtungen sollten möglich sein. 


\begin{tabular}{|l|l|c|}
\hline \multicolumn{1}{|c|}{ Indikator (Gewichtung) } & \multicolumn{1}{|c|}{ Abstufung } & Skala \\
\hline Landschaft (2) & Heißländen & 1 \\
\hline & Wald \& Heißlände & 2 \\
\hline & Nur Wald/Wiese \& Wald/Acker & 3 \\
\hline & Nur Wiese & 4 \\
\hline Wegegrün (2) & Allee & 1 \\
\hline & Baumreihe & 2 \\
\hline & Kein Grün & 3 \\
\hline & Einzelbaum & 4 \\
\hline Wegbreite (1) & $4 \mathrm{~m}$ & 1 \\
\hline & $3 \mathrm{~m}$ & 2 \\
\hline & $2 \mathrm{~m}$ & 4 \\
\hline & $0,8 \mathrm{~m}$ & 1 \\
\hline Gewässer (2) & Altarm klein, 0 \% Schilf & 2 \\
\hline & Altarme klein und sonstige Gewässertypen & 3 \\
\hline & Altarm groß & 4 \\
\hline & Kein Gewässer & 1 \\
\hline Erholungserlebnis (1) & Obere Lobau & 4 \\
\hline & Untere Lobau & 5 \\
\hline
\end{tabular}

Tab. 42: Indikatoren zur Bewertung der Sensibilität der Lobau (Skala: $1=$ nicht sensibel; $5=$ sehr hohe Sensibilität gegenüber hohen Besucherströmen).

Die Punkte 1 bis 5 wurden hinsichtlich ihrer Bedeutung zur Bewertung der Sensibilität gewichtet. So wurde beispielsweise den Landschaftselementen eine höhere Bedeutung zugemessen als der Wegbreite. Die Bedeutungszumessung resultierte u.a. auch aus den Ergebnissen des Wahlmodells: Die Landschaftselemente hatten eine höhere Bedeutung für die Wahlentscheidungen der Befragten als beispielsweise die Wegetypen. Diese Indikatorenwerte wurden dann aggregiert und in einem weiteren Schritt jeder Rasterzelle der Lobau zugewiesen (Abb. 108). 


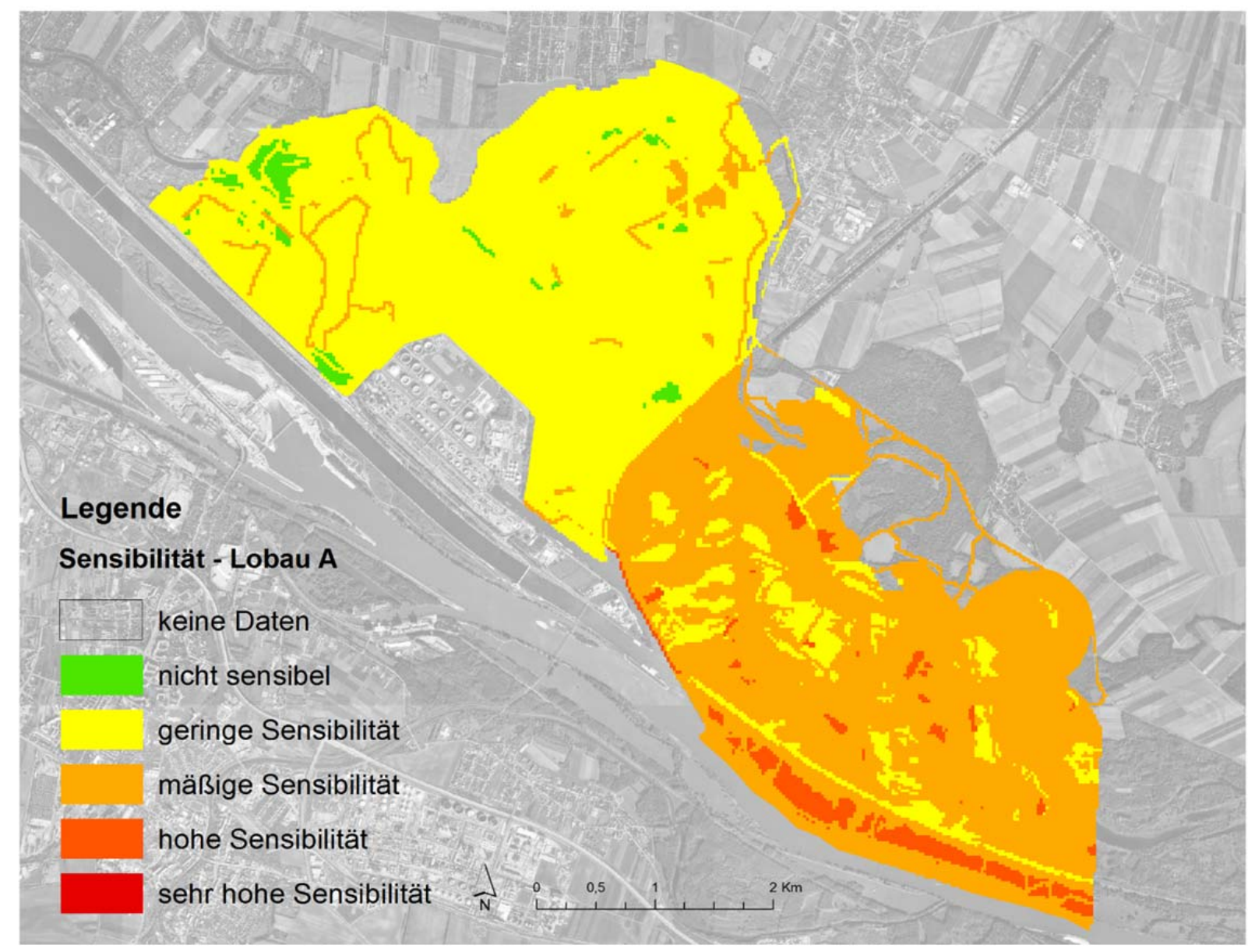

Abb. 108: Empfindlichkeit der Lobau (Sensibilität) gegenüber hohen Besucherströmen

\subsubsection{Auswirkungen des prognostizierten Besucherdrucks auf die Erholungsqualität}

Werden keine Maßnahmen zur Reduktion des Besucherdrucks gesetzt, so wird bei der prognostizierten Verdoppelung der Besuchsbelastung die Erholungsqualität in der Lobau deutlich beeinträchtigt. Sensible Bereiche, wie Offenlandbereiche, z.B. Auwiesen, die von Wegen durchzogen sind, oder die Untere Lobau, werden in Folge deutlich geringere Erholungsqualitäten bieten (Abb. 109). Auf schmalen Wegen werden vermehrt Nutzerkonflikte auftreten. Höhere Besuchermengen werden nicht nur den schon jetzt für die Wochenenden festgestellten Überfüllungseindruck erhöhen, sie werden auch verstärkt Konflikte zwischen den Erholungssuchenden selbst nach sich ziehen. Das Ausweichverhalten der Erholungssuchenden wird sich intensivieren. Bisher weniger stark besuchte Bereiche der Lobau werden künftig einer stärkeren Erholungsnutzung unterworfen sein. Hohe Besuchermengen werden auch zu neuen Trampelpfaden und verstärkten Wegeerosionen führen. Konflikte mit dem Naturraum, u.a. aufgrund einer steigenden Anzahl an Hunden in der Lobau, werden zunehmen. Die Lobau, v.a. die Obere Lobau, wird sich weiter in Richtung eines innerstädtischen Erholungsbietes entwickeln. Eine ähnliche Entwicklung ist ebenso für Teilbereiche der Unteren Lobau absehbar. 


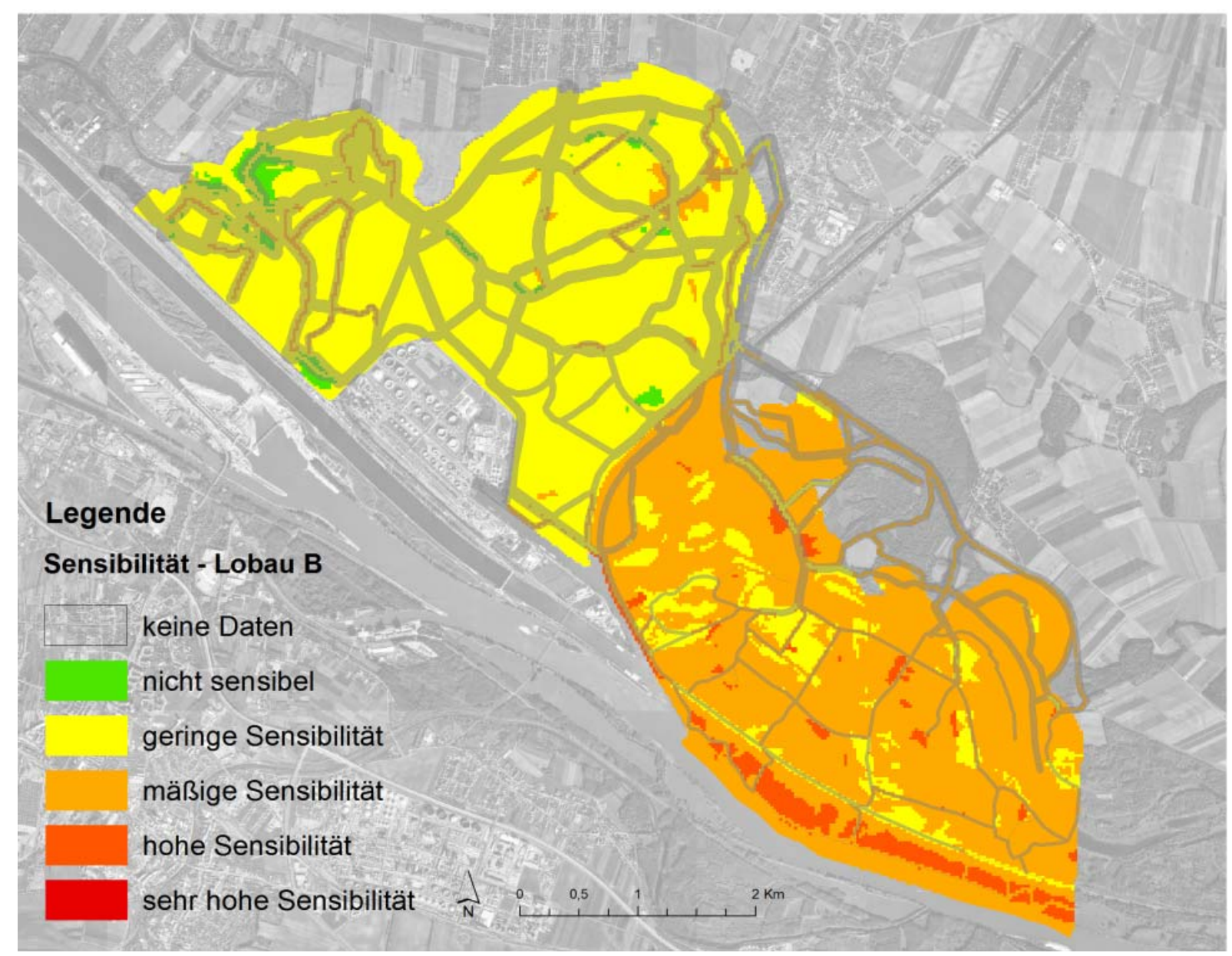

Abb. 109: Überlagerung der Empfindlichkeit der Lobau (Sensibilität) mit den Nutzungsintensitäten eines Spitzentages

\subsubsection{Entlastung der Lobau durch die Pufferwirkung des Lobau-Vorlandes}

Die zentrale Fragestellung des Forschungsprojektes war, inwieweit eine landschaftliche Ausgestaltung des Lobau-Vorlandes die Besuchsbelastung in der Lobau reduzieren kann. Die Ergebnisse zeigten, dass bei einer Attraktivierung des Vorlandes rund $30 \%$ weniger Besucherkilometer/Tag in der Lobau gegenüber dem „Laissez-faire -Szenario“ (2025) zu verzeichnen sind, dies gilt sowohl für Werk- wie Spitzentage (Abb. 110). Dennoch bedeutet dies immer noch eine 1,6-fache Erhöhung der Besuchsbelastung gegenüber dem Jahr 1999.

Die Pufferwirkung wirkt aufgrund der Konzentration der Maßnahmen im Bereich Saltenstraße v.a. auf die Obere Lobau. Die Untere Lobau wird hinsichtlich Besucherkilometer kaum entlastet. Dadurch ergibt sich je nach Szenario eine 2,4 bis 3,1-fache Erhöhung der Besuchsbelastung in der Unteren Lobau gegenüber 1999. In der Oberen Lobau ist eine 1,3 bis 1,5-fache Erhöhung gegenüber 1999 anzunehmen. Vor allem die Besucherzunahme an Werktagen ist kritisch, da diese eine hohe Eintrittshäufigkeit aufweisen.

Die Unterschiede hinsichtlich der Pufferwirkung auf die Lobau waren zwischen den vier Szenarien relativ gering (Tab. 43). Zwischen 27,6\% und 31,1\% der Besucherbelastung (Besucherkilometer) werden statt in der Lobau im Lobau-Vorland zurückgelegt. Die Pufferwirkung der flächigen Ausgestaltung ist um 2,2 bis 3,0\% besser als jene der linearen. 


\begin{tabular}{|l|l|l|}
\hline Szenarien & Spitzentag & Werktag \\
\hline $\begin{array}{l}\text { Lineare Ausgestaltung (ca. 12 \% mehr an Wegen gegenüber } \\
\text { Istzustand) }\end{array}$ & $27,9 \%$ & $28,3 \%$ \\
\hline $\begin{array}{l}\text { Flächige Ausgestaltung (ca. 14 \% mehr an Wegen gegenüber } \\
\text { Istzustand) }\end{array}$ & $30,4 \%$ & $30,5 \%$ \\
\hline $\begin{array}{l}\text { 1b + Lineare Ausgestaltung (ca. 8 \% mehr an Wegen gegenüber } \\
\text { Istzustand) }\end{array}$ & $27,6 \%$ & $28,8 \%$ \\
\hline $\begin{array}{l}\text { 1b + Flächige Ausgestaltung (ca. 11 \% mehr an Wegen gegenüber } \\
\text { Istzustand) }\end{array}$ & $30,6 \%$ & $31,1 \%$ \\
\hline
\end{tabular}

Tab. 43: Pufferwirkung je nach Szenario

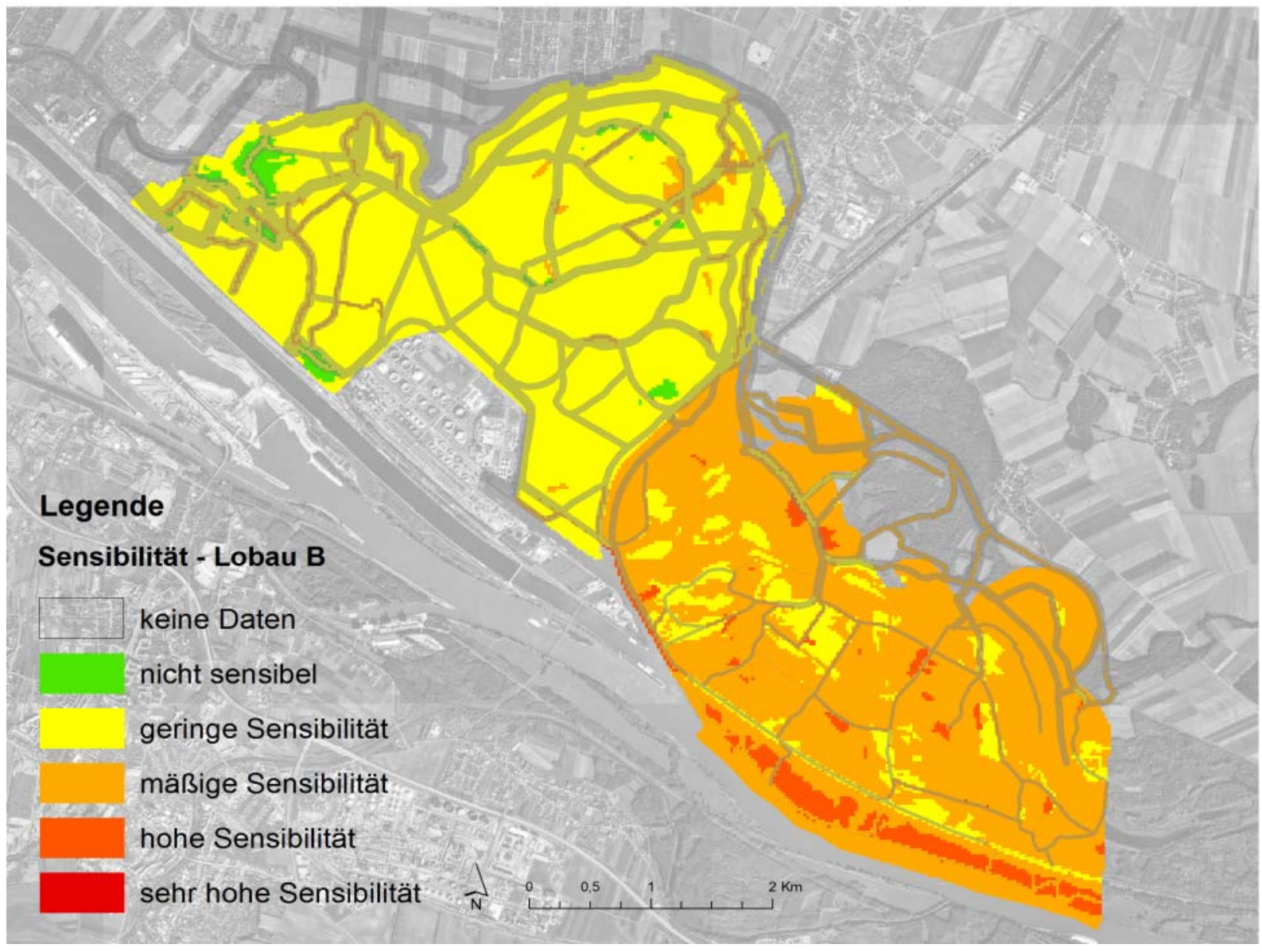

Abb. 110: Überlagerung der Empfindlichkeit der Lobau (Sensibilität) mit den Nutzungsintensitäten eines Spitzentages basierend auf Szenario „flächig ohne $1 b^{\prime \prime}$

\subsubsection{Bewertung der Szenarien}

Die lineare Ausgestaltung erhöht die Attraktivität des Lobau-Vorlandes, allerdings ist hier die Ausgestaltung v.a auf die Präferenzen der Radfahrenden ausgerichtet. Erst die flächige Ausgestaltung des Vorlandes mit einer Wald-Wiesen-Mosaiklandschaft und Fußgängerwegen entspricht den Präferenzen vieler Erholungssuchenden. Dennoch kann die flächige Ausgestaltung die Besuchermenge in der Lobau nicht wesentlich stärker reduzieren als die lineare Ausgestaltung, sie ist aber aus stadtökologischer Sicht vorteilhaft. Gleichzeitg führt diese Umgestaltung des Lobau-Vorlandes zu einem Verlust von ackerbaulichen Flächen im direkten Nahbereich zu den Konsumenten.

Das Gewässerszenario (1b) hat nahezu keine Auswirkungen hinsichtlich einer Pufferwirkung der Besuchszahlen. Es ermöglicht aber eine starke Beruhigung von Bereichen der Unteren Lobau v.a. an Spitzentagen und bedeutet eine gewässerökologische Aufwertung des Gebietes und damit langfristig eine positive Entwicklung vor allem der aquatischen und halb-aquatischen Lebensräume. Allerdings ist die Zugänglichkeit des Gebietes für Erholungssuchende stark eingeschränkt und führt damit zu einer Konzentration der Erholungssuchenden auf die restlichen Bereiche der Unteren Lobau. 
Gleichzeitig ermöglicht dieser Bereich spezifische Erholungsqualitäten wie Einsamkeit und vermehrte Wildtiersichtungen. Aus Sicht der Erholungsplanung kommt dem Szenario flächig ohne 1b daher die größte Bedeutung zu.

\subsection{Verkehrliche Wirkungen außerhalb der Lobau}

Im Fall der LobaubesucherInnen führt die Attraktivierung des Verkehrssystems nur beschränkt zu einer Veränderung der Verkehrsmittelwahl bei der Anreise. Im Nahbereich wohnende LobaubesucherInnen nutzen bereits jetzt zu einem sehr hohen Anteil das Fahrrad als Anreisemittel (65\% der im Lobau-Vorland wohnenden, $41 \%$ der BewohnerInnen des übrigen 22. Bezirks und immerhin $13 \%$ der BewohnerInnen der übrigen Wiener Gemeindebezirke). $24 \%$ der BewohnerInnen des Lobau-Vorlands besuchen die Lobau zu Fuß (Tab. 44).

\begin{tabular}{|l|l|l|l|l|}
\hline & zu Fuß & mit dem Rad & Bus, Bahn & MIV (PKW) \\
\hline 22. Bezirk, Lobau-Vorland & 24 & 65 & 0 & 11 \\
\hline 22. Bezirk ohne Lobau-Vorland & 6 & 41 & 5 & 48 \\
\hline Wien übrige Bezirke & 1 & 13 & 16 & 70 \\
\hline Groß-Enzersdorf & 20 & 60 & 0 & 20 \\
\hline übrige Region & 0 & 4 & 0 & 96 \\
\hline Insgesamt & 11 & 41 & 6 & 43 \\
\hline
\end{tabular}

Tab. 44 : Anreiseverkehrsmittewahl der LobaubesucherInnen. Quelle: ÖIR, Hochrechnung der Erhebung 2000.

Die Anreise mit einem öffentlichen Verkehrsmittel spielt bei längeren Distanzen eine Rolle (16\% der aus Wien ohne 22. Bezirk anreisenden Lobaubesucherinnen), nicht aber im Nahbereich. Hier dürften sowohl das Bewegungsbedürfnis (Radfahrende) als auch die ungünstige Erreichbarkeit der Lobau im ÖV (PKW-Anreisende) die zentralen Motive sein. Vor dem Hintergrund des hohen Radfahranteils im Lobau-Vorland kann davon ausgegangen werden, dass sich jene Maßnahmen, die das Radfahren im Vorland attraktivieren, weniger auf die Wahl des Anreiseverkehrsmittels auswirken als auf die Routenwahl der Radfahrer (fahren mehr im Vorland, weniger innerhalb der Lobau).

Die „globalen“ Umweltwirkungen (Schadstoffemissionen, $\mathrm{CO}_{2}$ ) können damit nahezu ausschließlich an der durch die Maßnahmen möglichen Reduzierung der MIV-Anreise gemessen werden; diese werden in Folge quantifiziert.

Maßnahmen im Lobau-Vorland haben auch Auswirkungen auf die Umweltsituation außerhalb der Lobau. Aus den Erhebungen der Lobau-BesucherInnen sind deren räumlich lokalisierte Herkunft und ihr Anreise-Verkehrsmittel bekannt. Im Verkehrsmodell Wien-Ostregion des ÖIR konnten somit auch die Auswirkungen des Szenarios Parkraum-Management auf Verkehrsverhalten und Schadstoffausstoß der Lobau-BesucherInnen simuliert werden. Unterstellt werden die Auflassung bzw. Verlegung von Parkplätzen sowie die Unterbrechung von Schleichwegen für den motorisierten Individualverkehr (Szenario A).

Die Modellrechnung zeigt, dass die aktuelle Verkehrsmittelwahl bei der Anreise zur Lobau (49\% MIV, $33 \%$ Fahrrad, $12 \%$ zu Fuß, $6 \%$ ÖV) bis 2025 sich ohne Parkraummanagement moderat (Szenario B) und mit Parkraummanagement (Szenario A) stärker zugunsten umweltfreundlichen Verkehrsverhaltens verändern dürfte (Abb. 111, Abb. 112 und Abb. 113): 


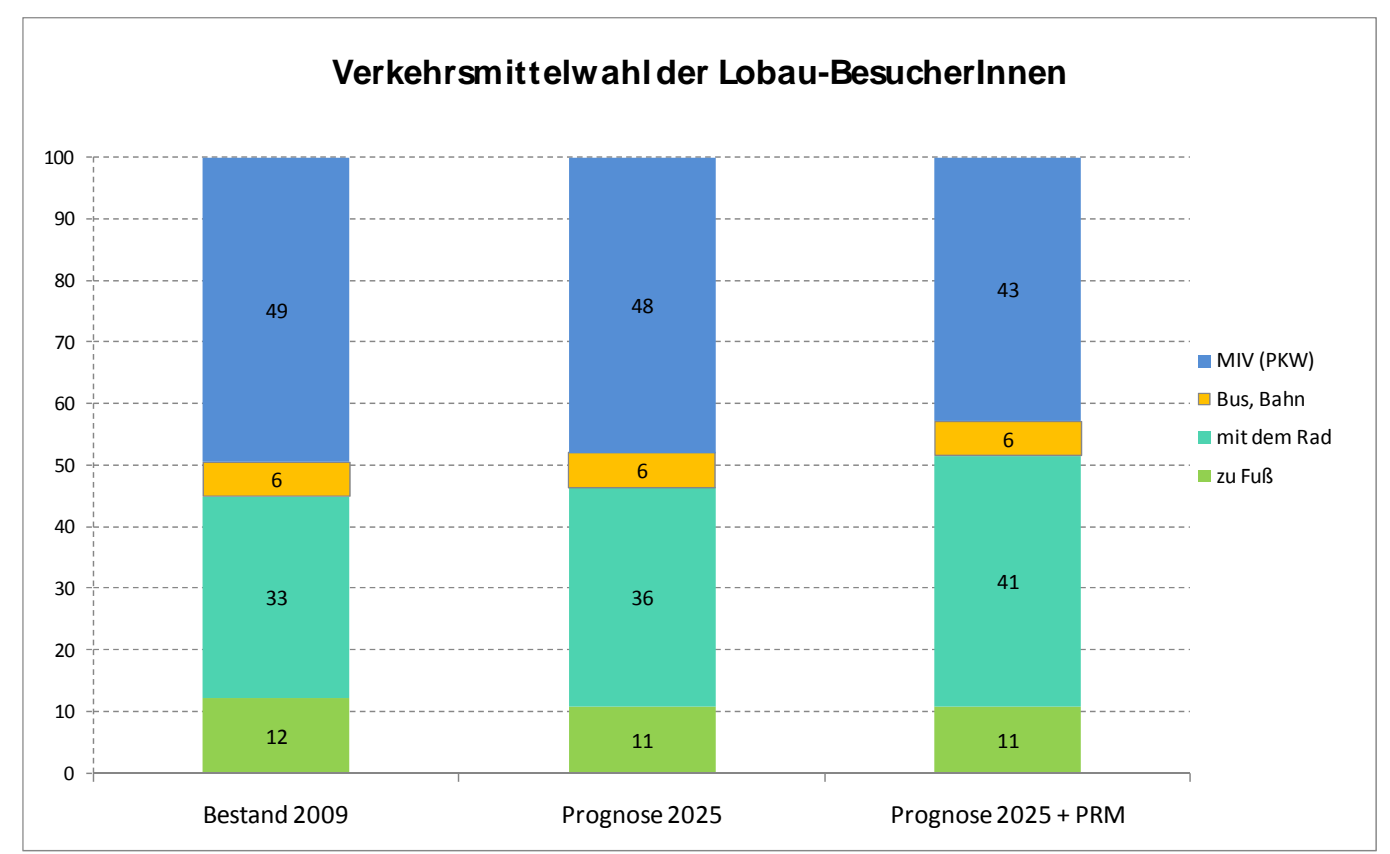

Abb. 111: Veränderung der Verkehrsleistung durch Vekehrsszenarien. Quelle: ÖIR, eigene Berechnung, Verkehrsmodell WienOstregion. (PRM = Parkraummanagement)

Der Anteil des Radverkehrs steigt dann von $36 \%$ (Szenario B) auf 41 \% (Szenario A), der MIV würde von $48 \%$ auf $43 \%$ zurückgehen. Die Verkehrsleistung (KFZ-km) der Lobau-BesucherInnen schrumpft damit (in gesamt Wien und Umland) um $12 \%$, die $\mathrm{CO}_{2}$-Emissionen um $14 \%$ (Zeithorizont 2025).

\section{Parkraummanagement: Veränderung der Verkehrsleistung} [Mio. PKW-km der Lobaubesucher/Jahr]

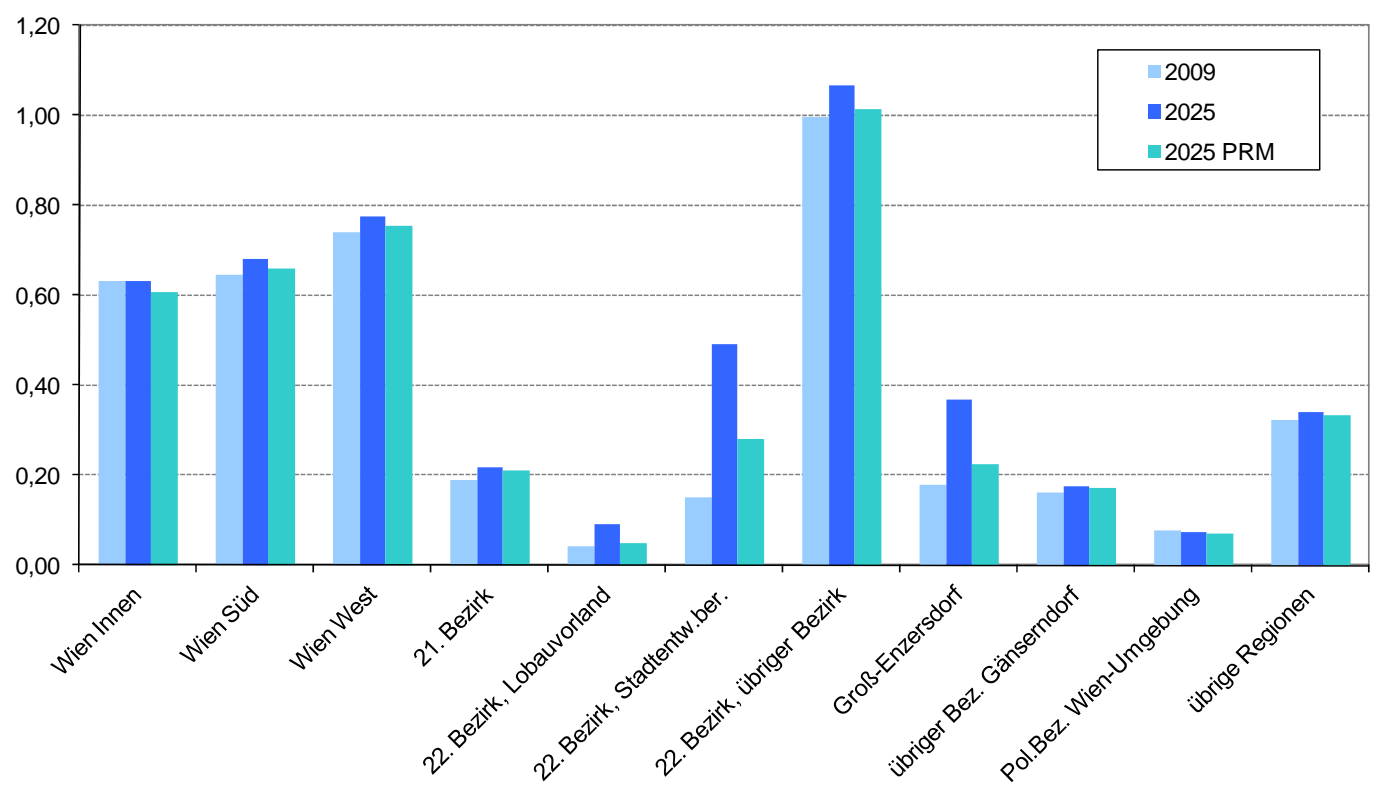

Abb. 112: Veränderung der Verkehrsleistung durch Vekehrsszenarien. Quelle: ÖIR, eigene Berechnung, Verkehrsmodell WienOstregion. (PRM = Parkraummanagement) 


\section{Parkraummanagement: Veränderung der CO2-Emissionen [t CO2 der Lobaubesucher/Jahr]}

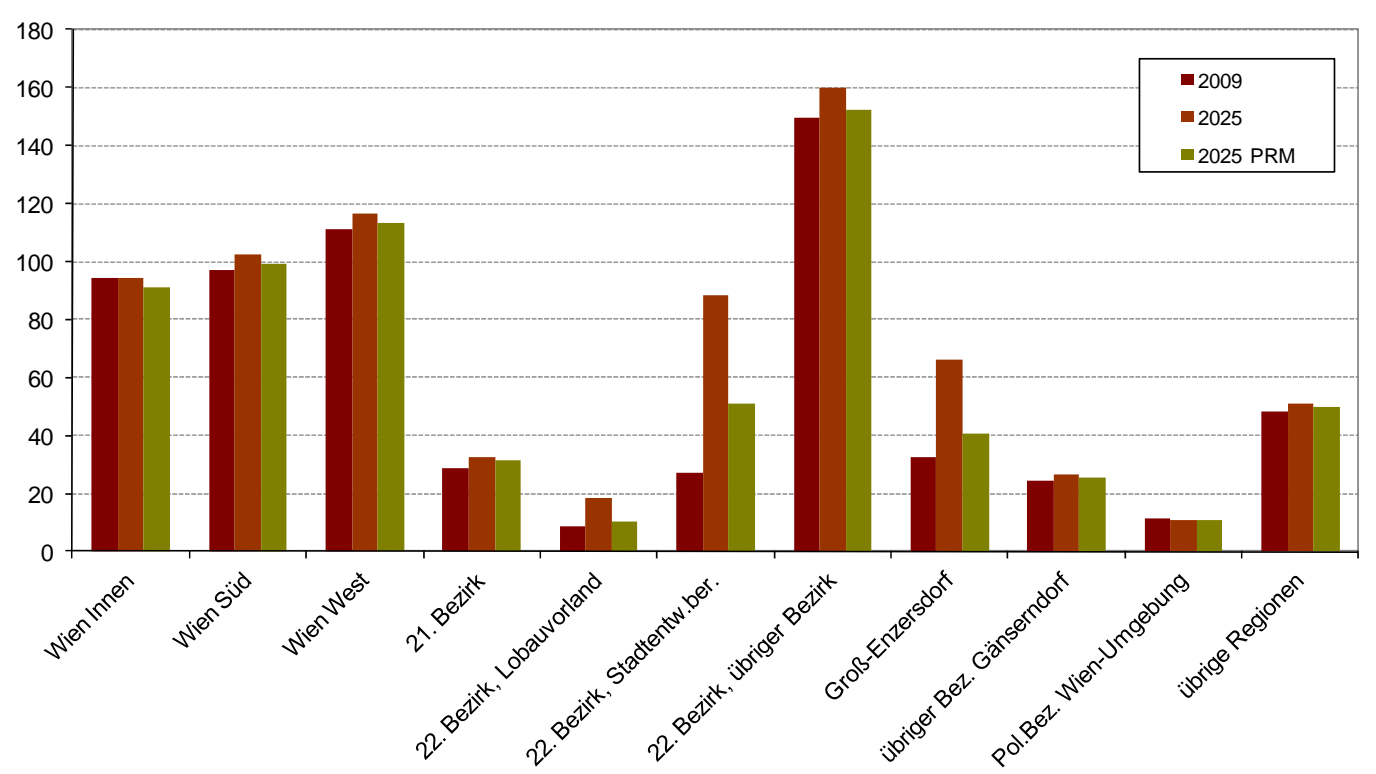

Abb. 113: Umweltwirkungen der Verkehrsszenarien. Quelle: ÖIR, eigene Berechnung, Verkehrsmodell Wien-Ostregion. (PRM = Parkraummanagement)

\subsection{Synthese der Kriterien}

Die einzelnen Fachbereiche bewerteten die Szenarien anhand der definierten Kriterien. Daraus ergab sich eine Reihenfolge der vier Szenarien hinsichtlich ihrer Auswirkungen aus Sicht des jeweiligen Fachbereichs (1=beste Reihung; Tab. 45).

\begin{tabular}{|c|c|}
\hline Szenario & Beurteilung der Fachbereiche \\
\hline $\begin{array}{l}\text { Szenario linear } \\
\text { ohne } 1 \text { b }\end{array}$ & $\begin{array}{l}\text { Vegetationsökologie } \\
\text { Vorteile: keine } \\
\text { Nachteile: } \\
\text { - stetige Zunahme der Verlandung } \\
\text { Kritische Bereiche: } \\
\text { * Uferbereiche - Röhricht } \\
\text { Reihung innerhalb der Szenarien: } 4\end{array}$ \\
\hline & $\begin{array}{l}\text { Gewässerökologie } \\
\text { Vorteile: } \\
\text { + Obwohl die Vorlandgestaltung nur sehr geringe Auswirkungen auf die } \\
\text { Gewässerökologie des Gesamtgebietes hat, ist doch der Besucherdruck auf die } \\
\text { Badegewässer (Dechantlacke, Panozzalacke) vermindert. } \\
\text { Nachteile: } \\
\text { - Verglichen mit dem derzeitigen Zustand (Modellergebnis 1999) zeigt sich eine } \\
\text { deutliche Verschlechterung der ökologischen Funktionsfähigkeit der Unteren Lobau. } \\
\text { Kritische Bereiche: } \\
\text { * Badegewässer Dechantlacke und Panozzalacke, Groß-Enzersdorfer-Arm } \\
\text { Reihung innerhalb der Szenarien: } 3\end{array}$ \\
\hline
\end{tabular}




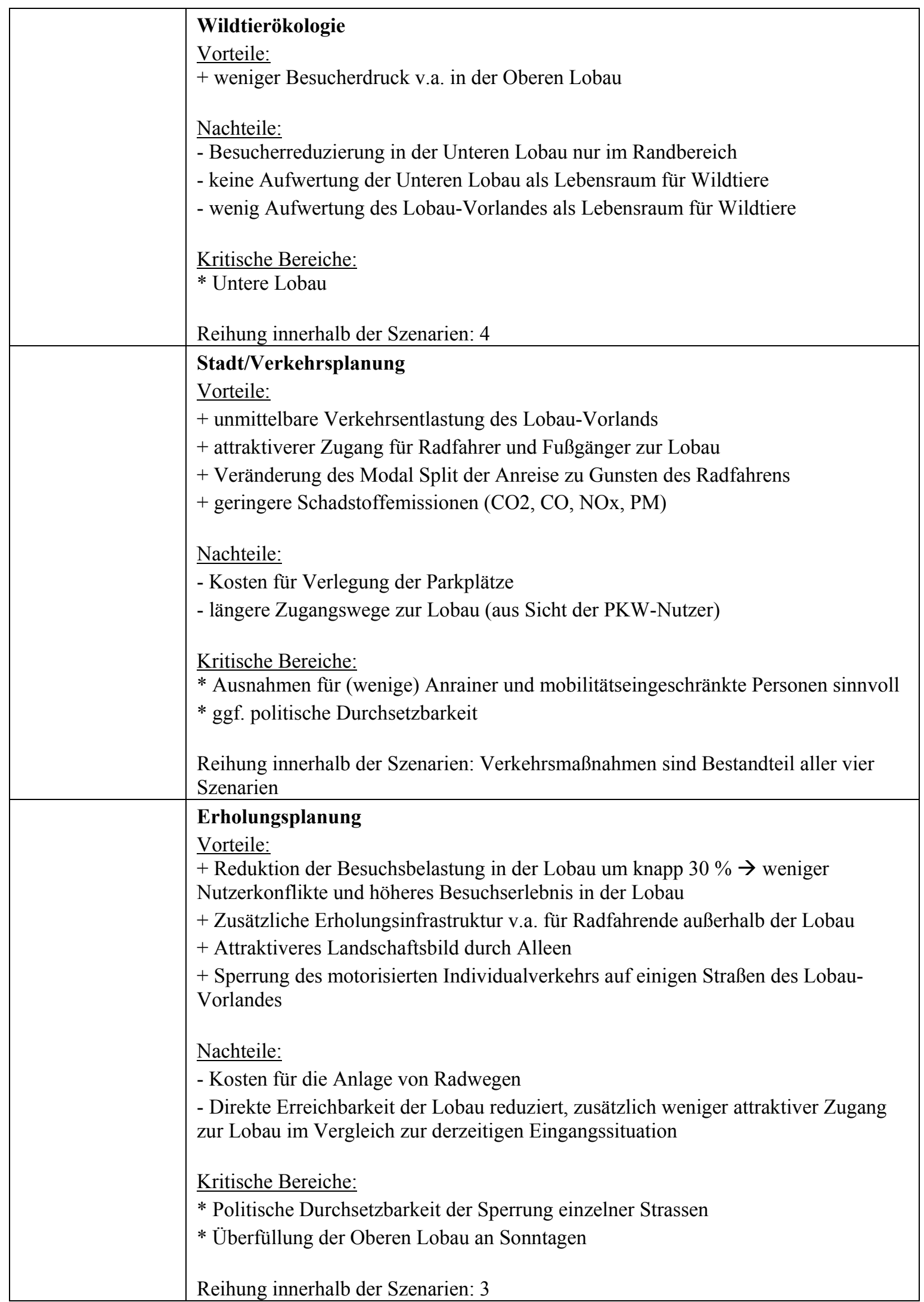




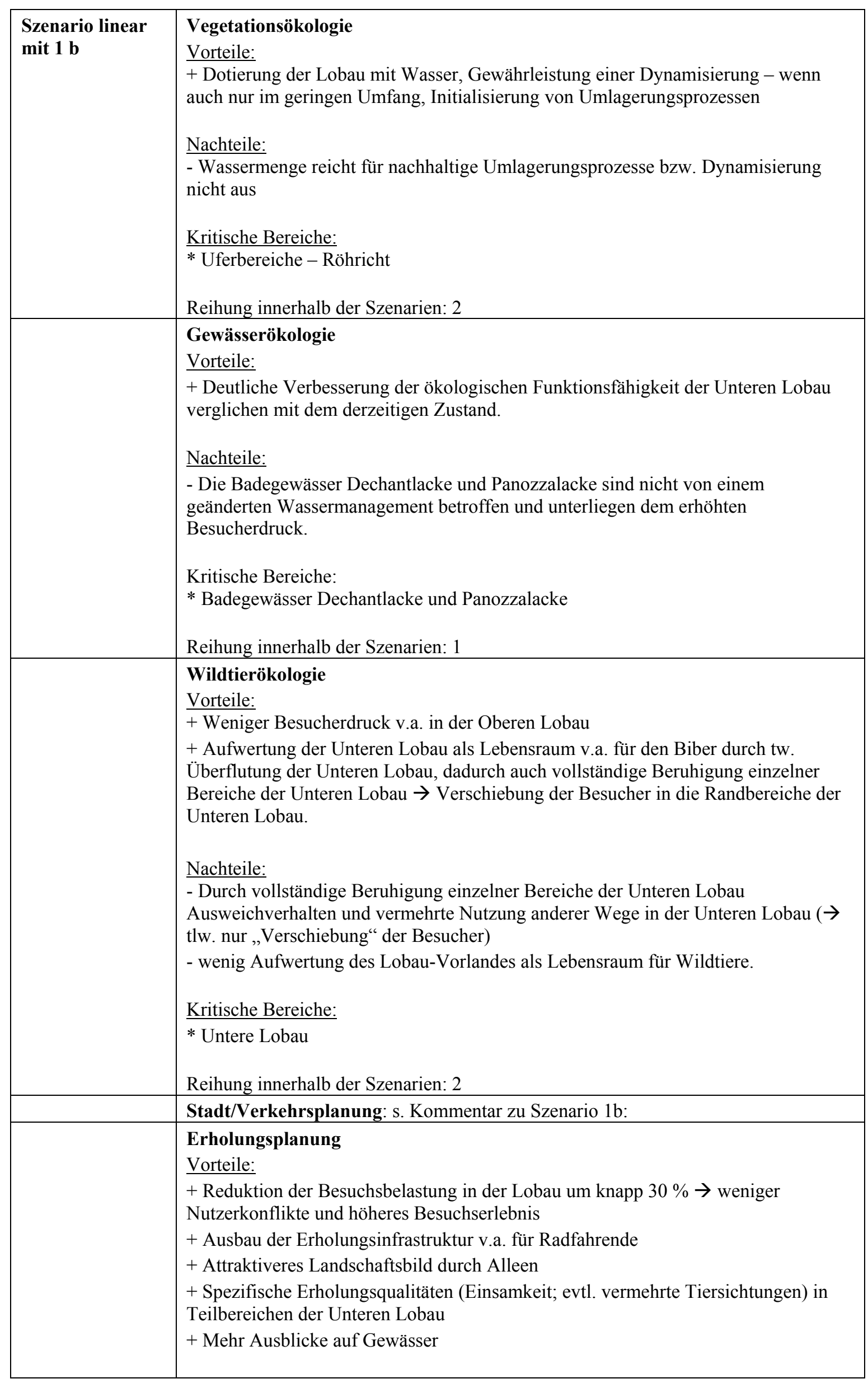




\begin{tabular}{|c|c|}
\hline & $\begin{array}{l}\text { Nachteile: } \\
\text { - Erschwerte Zugänglichkeit von Teilbereichen in der Unteren Lobau, vermehrt } \\
\text { Stichwege } \\
\text { - Konzentration der Erholungssuchenden auf restliche Bereiche der Unteren Lobau } \\
\text { Kritische Bereiche: } \\
\text { * Überfüllung der Oberen Lobau an Sonntagen } \\
\text { Reihung innerhalb der Szenarien: } 4\end{array}$ \\
\hline \multirow[t]{4}{*}{$\begin{array}{l}\text { Szenario flächig } \\
\text { ohne } 1 \text { b }\end{array}$} & $\begin{array}{l}\text { Vegetationsökologie } \\
\text { Vorteile: } \\
\text { + Keine erkennbaren außer einer geringfügigen Abnahme der Beeinträchtigungen der } \\
\text { Vegetation an Wegränder in der Oberen Lobau } \\
\text { Nachteile: } \\
\text { - fehlende Dynamisierung } \\
\text { Kritische Bereiche: } \\
\text { * Uferbereiche } \\
\text { Reihung innerhalb der Szenarien: } 3\end{array}$ \\
\hline & $\begin{array}{l}\text { Gewässerökologie } \\
\text { Vorteile: } \\
\text { + Obwohl die Vorlandgestaltung nur sehr geringe Auswirkungen auf die } \\
\text { Gewässerökologie des Gesamtgebietes hat, ist doch der Besucherdruck auf die } \\
\text { Badegewässer (Dechantlacke, Panozzalacke) vermindert. } \\
\text { Nachteile: } \\
\text { - Verglichen mit dem derzeitigen Zustand (Modellergebnis 1999) zeigt sich eine } \\
\text { deutliche Verschlechterung der ökologischen Funktionsfähigkeit der Unteren Lobau. } \\
\text { Kritische Bereiche: } \\
\text { * Badegewässer Dechantlacke und Panozzalacke, Groß-Enzersdorfer-Arm } \\
\text { Reihung innerhalb der Szenarien: } 3\end{array}$ \\
\hline & $\begin{array}{l}\text { Wildtierökologie } \\
\text { Vorteile: } \\
\text { + Weniger Besucherdruck v.a in der Oberen Lobau } \\
\text { + Aufwertung des Lobau-Vorlandes als Lebensraum für Wildtiere (Wald-Wiesen- } \\
\text { Landschaft) } \\
\text { Nachteile: } \\
\text { - Keine Aufwertung der Unteren Lobau als Lebensraum für Wildtiere. } \\
\text { Kritische Bereiche: } \\
\text { * Untere Lobau } \\
\text { Reihung innerhalb der Szenarien: } 3\end{array}$ \\
\hline & Stadt/Verkehrsplanung: s. Kommentar zu Szenario 1b \\
\hline
\end{tabular}




\begin{tabular}{|c|c|}
\hline & $\begin{array}{l}\text { Erholungsplanung } \\
\text { Vorteile: } \\
\text { + Reduktion der Besuchsbelastung in der Lobau um } 30 \% \rightarrow \text { weniger } \\
\text { Nutzerkonflikte und höheres Besuchserlebnis } \\
\text { + Zusätzliche Erholungsinfrastruktur für Radfahrende und zu Fuß Gehende außerhalb } \\
\text { der Lobau } \\
\text { + Sehr attraktives Landschaftsbild durch Alleen und Wald-Wiesen-Flächen } \\
\text { + Sperrung des motorisierten Individualverkehrs auf einigen Straßen des Lobau- } \\
\text { Vorlandes } \\
\text { Nachteile: } \\
\text { - Großer Flächenbedarf (Verlust von ackerbaulichen Flächen) } \\
\text { - Umwandlung von Feldern in Wiesen und Waldkompartimente } \\
\text { - Raumkante (Pappelkulisse) der Lobau weniger erlebbar } \\
\text { - Kosten für die Landnutzungsänderung und Anlage von Rad- und Fußwegen } \\
\text { - Attraktiverer Zugang zur Lobau als lineares Szenario } \\
\text { Kritische Bereiche: } \\
\text { * Politische Durchsetzbarkeit und Kosten der Ausgestaltung } \\
\text { * Überfüllung der Oberen Lobau an Sonntagen } \\
\text { Reihung innerhalb der Szenarien: } 1\end{array}$ \\
\hline $\begin{array}{l}\text { Szenario flächig } \\
\text { mit } 1 \text { b }\end{array}$ & $\begin{array}{l}\text { Vegetationsökologie } \\
\text { Vorteile: } \\
\text { + Dotierung der Lobau mit Wasser, Gewährleistung einer Dynamisierung - wenn } \\
\text { auch nur im geringen Umfang, Initialisierung von Umlagerungsprozessen verbunden } \\
\text { mit einer Abnahme des Besucherdrucks in der Oberen Lobau. } \\
\text { Nachteile: keine erkennbar } \\
\text { Kritische Bereiche: } \\
\text { * Uferbereiche } \\
\text { Reihung innerhalb der Szenarien: } 1\end{array}$ \\
\hline & $\begin{array}{l}\text { Gewässerökologie } \\
\text { Vorteile: } \\
\text { + Deutliche Verbesserung der ökologischen Funktionsfähigkeit der Unteren Lobau } \\
\text { verglichen mit dem derzeitigen Zustand. } \\
\text { Nachteile: } \\
\text { - Die Badegewässer Dechantlacke und Panozzalacke sind nicht von einem } \\
\text { geänderten Wassermanagement betroffen und unterliegen dem erhöhten } \\
\text { Besucherdruck. } \\
\text { Kritische Bereiche: } \\
\text { * Badegewässer Dechantlacke und Panozzalacke } \\
\text { Reihung innerhalb der Szenarien: 1 }\end{array}$ \\
\hline
\end{tabular}




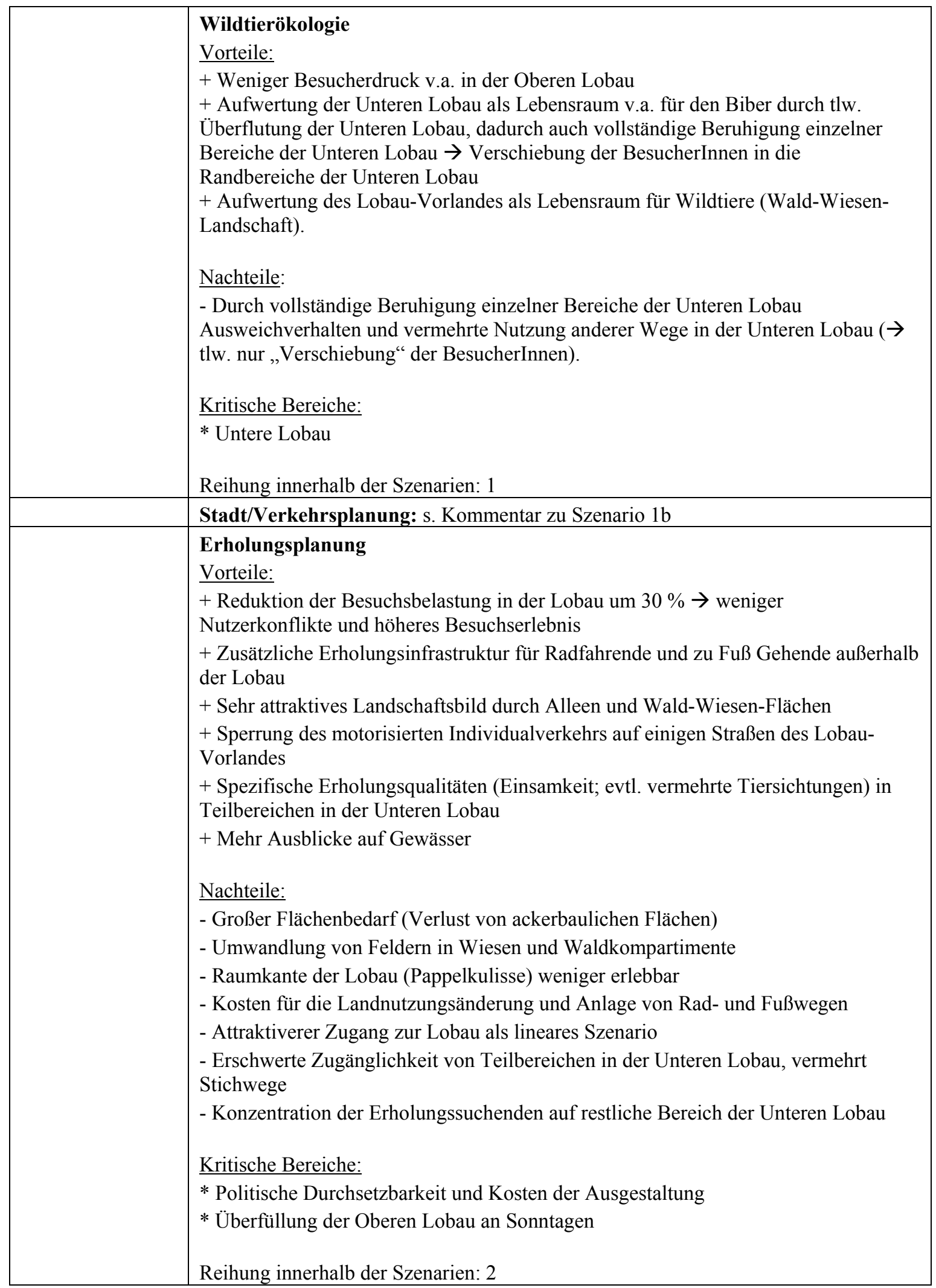

Tab. 45: Zusammenfassende Bewertung und Vergleich der vier Szenarien nach Fachbereichen 


\section{Diskussion und Vorschläge für Managementmaßnahmen}

\subsection{Zusammenfassende Bewertungen der Szenarien}

Die städtebauliche Entwicklung wird zu einer Zunahme der lokalen Bevölkerung im Umkreis der Lobau führen. An die 50.000 neue EinwohnerInnen, das entspricht der Einwohnerzahl von St. Pölten, werden dafür sorgen, dass der Erholungsdruck auf die Lobau drastisch steigen wird. Einige Forschende gehen davon aus, dass schon jetzt die soziale und ökologische Tragfähigkeit der Lobau erreicht, und an schönen Wochenendtagen zumindest die soziale Tragfähigkeit für viele BesucherInnnen überschritten ist. Auch wenn durch die hohen Besucherströme das Ökosystem Au nicht grundsätzlich gefährdet ist, zumindest aus vegetationsökologischer Sicht, so sind weitere Beeinträchtigungen des Naturraums und der Erholungsqualität $\mathrm{zu}$ erwarten. Besucherlenkungsmaßnahmen sind Instrumente die normalerweise angewendet werden, um negative Auswirkungen des Besucherdrucks $\mathrm{zu}$ minimieren. Aufgrund des prognostizierten Anstiegs der Besuchermenge in die Lobau ist aber zu bezweifeln, dass Lenkungsmaßnahmen alleine ausreichen werden, um die negativen Auswirkungen des Besucherzuwachses effektiv zu reduzieren. Die Nationalparkwürdigkeit der Lobau ist eventuell bedroht.

Ziel der vorliegenden Studie war es daher, Maßnahmen zu entwickeln die diesen prognostizierten starken Besucherdruck reduzieren können und deren Effektivität zu simulieren. Die Maßnahmen fokussierten dabei auf das Lobau-Vorland im Wiener Bereich, da hier der Schwerpunkt der städtebaulichen Entwicklung liegt. Die Überlegung war, dass eine Umwandlung des agrarisch geprägten und derzeit für die Erholungsnutzung unattraktiven Raumes in eine Erholungslandschaft und die gleichzeitige Zurückdrängung des motorisierten Individualverkehrs, die Besucherbelastung im Schutzgebiet reduzieren würden, da viele Besucherkilometer bereits im Vorland und nicht in der Lobau zurückgelegt werden.

Dazu wurden vier Grundszenarien entwickelt, die sich hinsichtlich der Intensität der Ausgestaltung des Lobau-Vorlandes und einer Dynamisierung der Aulandschaft unterscheiden. Die Simulationen zeigten, dass die Szenarien rund $30 \%$ der Besuchsbelastung bei einer zusätzlichen Wegeinfrastruktur von ca. 12 bis $14 \mathrm{~km}$ im Lobau-Vorland absorbieren können. Die Rangreihung der Szenarien zeigte, dass aus ökologischer Sicht das Szenario flächige Ausgestaltung mit 1b (Gewässeranbindung an die Donau) am höchsten bewertet wurde (Tab. 46). Aus Sicht der Erholungsplanung war eine flächige Ausgestaltung ohne Gewässeranbindung das am höchsten bewertete Szenario.

\begin{tabular}{|l|c|c|c|c|}
\hline & $\begin{array}{c}\text { Szenario lineare } \\
\text { Ausgestaltung } \\
\text { ohne 1b } \\
\text { (Gewässer- } \\
\text { anbindung) }\end{array}$ & $\begin{array}{c}\text { Szenario lineare } \\
\text { Ausgestaltung } \\
\text { mit 1b } \\
\text { (Gewässer- } \\
\text { anbindung) }\end{array}$ & $\begin{array}{c}\text { Szenario flächige } \\
\text { Ausgestaltung } \\
\text { ohne 1b } \\
\text { (Gewässer- } \\
\text { anbindung) }\end{array}$ & $\begin{array}{c}\text { Szenario flächige } \\
\text { Ausgestaltung } \\
\text { mit 1b } \\
\text { (Gewässer- } \\
\text { anbindung) }\end{array}$ \\
\hline Vegetationsökologie & 3 & 2 & 3 & 1 \\
\hline Gewässerökologie & 3 & 1 & 3 & 1 \\
\hline Wildtierökologie & 4 & 3 & 2 & 1 \\
\hline $\begin{array}{l}\text { Stadt-, } \\
\text { Verkehrsplanung }\end{array}$ & & & & 2 \\
\hline Erholungsplanung & 3 & 4 & 1 & \\
\hline
\end{tabular}

Tab. 46: Rangreihung der Szenarien aus Sicht der Einzeldisziplinen (1=beste Reihung)

\subsection{Empfehlungen aus Sicht der Stadt- und Erholungsplanung}

Trotz der vorgeschlagenen Maßnahmen wird die Lobau noch mehr unter Erholungsdruck kommen. Vor allem die Besucherzunahme an Werktagen ist kritisch, da diese eine hohe Eintrittshäufigkeit aufweisen. Dies ist besonders für die Obere Lobau problematisch, da diese schon jetzt teilweise über ihre Tragfähigkeitsgrenze belastet ist (Arnberger et al., 2009). Die starke Erhöhung der Besuchsbelastung des bisher weniger intensiv genutzten BP Untere Lobau ist ebenfalls kritisch zu sehen. Neben der Zunahme von Besuchen war auch ein Ausweichverhalten der Agenten von der Oberen in die Untere Lobau aufgrund der höheren Besuchermengen in der Oberen Lobau 
festzustellen. Dies könnte in Folge auch für die Untere Lobau eine Überschreitung der Tragfähigkeitsgrenzen an den Wochenenden bedeuten.

Ein engmaschigeres Wegenetz, und die Hineinnahme von weiteren Flächen des Lobau-Vorlandes, könnten die Pufferkapazität des Vorlandes noch weiter erhöhen. Dennoch erscheint die Pufferwirkung des Vorlandes als nicht ausreichend. Es stellt sich die Frage, ob die gänzliche und intensive Ausgestaltung des Gesamtvorlandes mit einer attraktiven Erholungsinfrastruktur die zusätzlichen potenziellen Besuchermengen überhaupt aufnehmen kann. Auf Basis dieses Projektes ergibt sich folglich ein Bedarf nach weiteren Erholungsgebieten im 22. Bezirk, insbesondere um das Stadterweiterungsgebiet und in der Gemeinde Groß-Enzersdorf. Erste Überlegungen seitens der Wiener Stadtplanung sehen neue Erholungsgebiete nördlich der Seestadt Aspern vor. Diese Maßnahme könnte sicherlich zu einer weiteren Besuchermengenentlastung der Lobau führen. Weitere Maßnahmenbündel wie neue Erholungsgebiete insbesondere für den 22. Wiener Gemeindebezirk und für die Gemeinde Groß-Enzersdorf sind in weiterer Folge zu definieren und hinsichtlich ihrer Auswirkungen auf die Lobau zu simulieren.

Gleichzeitig sind weitere Besucherlenkungsmaßnahmen in der Lobau, insbesondere in der Unteren Lobau, zu überlegen. Sollte ein attraktives Substitut Lobau-Vorland vorhanden sein, könnten auch restriktivere Maßnahmen in der Lobau angedacht werden und bestimmte Aktivitäten auf definierte Wege bzw. Lobaubereiche beschränkt bzw. bestimmte Verhaltensweisen stärker geahndet werden.

\subsection{Empfehlungen aus Sicht der Verkehrsplanung}

Ziele:

- Reduzierung des zunehmenden Besucherdrucks auf die Lobau

- Verringerung des motorisierten Individualverkehrs im Lobau-Vorland

- Veränderung des Modal Split der Anreise zu Gunsten des Radfahrens

- geringere Schadstoffemissionen (CO2, CO, NOx, PM) im Lobau-Vorland und generell

Maßnahmen:

Prinzip Nr. 1 verfolgen: Mit Fahrrad ist die Lobau vom Norden aus weit besser zu erreichen als mit motorisierten Individualverkehr; Prinzip Nr. 2 verfolgen: Naherholung in unmittelbarer Nähe der neuen Siedlungsgebiete stärken und die Verbindung zur Donauinsel attraktivieren, um den zunehmenden Besucherdruck auf die Lobau zu verringern;

- Attraktivierung der Erholungsqualität im Lobau-Vorland (Aspern - Eßling)

- Attraktive Verbindung für RadfahrerInnen/JoggerInnen und Inline-SkaterInnen von der Seestadt Aspern zur Donauinsel schaffen (Querung über Biberhaufenweg ist heute wenig attraktiv)

- Attraktive Verbindung für RadfahrerInnen von Seestadt Aspern zu den Badeteichen nördlich der Ostbahn (Badeteich Hirschstetten, etc. ...)

- Attraktivierung des Zugangs zu Lobau und Lobau-Vorland für RadfahrerInnen und FußgängerInnen (kein oder reduzierter MIV, Führung in Grünzone bzw. in Alleen)

- Gestaltung der Verbindung Seestadt Aspern - Lobau über die Saltenstraße als attraktive Radroute/Radweg (Reduzierung der Barrierewirkung des General MotorWerks Aspern)

- Schaffung einer attraktiven Radrouten/-wegverbindung zwischen dem Bereich Erzherzog-Karl-Stadt / U2 und der Lobau

- Reduzierung des motorisierten Individualverkehrs beim Zugang zur Lobau

- Erschwerung des Zugangs zu Lobau und Lobau-Vorland für den motorisierten Individualverkehr, Verringerung der Fahrtkilometer MIV im Lobau-Vorland

- Unterbrechung des Schleichwegs Lobaugasse - Brockhausengasse - Grohmannstraße - Kirschenallee im MIV

- Auflassung Parkplatz Luitpold-Sterngasse

- Verlegung Parkplatz Saltenstraße weiter ins Lobau-Vorland um ca. $600 \mathrm{~m}$ nach N

- Verlegung Parkplatz Eßlinger Furt um 200m nach W

- Sicherung der Mobilitätsbedürfnisse der AnrainerInnen 
- Intervallverdichtung der Buslinie 91A an aufkommensstarken Tagen (Sommersaison, Wochenenden) (Anmerkung: ein ÖV-Shuttle Seestadt - Lobaueingang dürfte nach den Mobiltätsanalysen nur wenig verkehrswirksam sein)

\subsection{Empfehlungen aus Sicht der Wildtierökologie}

Aus der Sicht der Wildbiologie ist das Szenario „Lobau B“ (Lobau mit Gewässerszenario 1b) plus „Vorland B“ (flächige Ausgestaltung des Lobau-Vorlandes) zu empfehlen. Die Ausgestaltung des Lobau-Vorlandes (linear oder flächig) hat in jedem Fall den Effekt, dass mehr Menschen das LobauVorland als Naherholungsgebiet nutzen würden, statt die Lobau aufzusuchen, wodurch sich der zu erwartende Besucherdruck auf die Lobau im Vergleich zum „Laissez-faire“-Szenario verringern würde. Eine lineare Ausgestaltung würde laut den Modellierungen v.a. die Radfahrenden ansprechen, die die größte Nutzergruppe in der Lobau ausmachen. Im Gegensatz dazu würde eine flächige Ausgestaltung nicht nur die RadfahrerInnen, sondern auch die HundebesitzerInnen ansprechen. Diese sind laut Befragungen nicht nur die drittgrößte Nutzergruppe in der Lobau, sondern stellen aus Sicht der Wildbiologie auch die problematischste Gruppe dar, da ein Großteil der Hunde nicht an der Leine geführt werden und freilaufende Hunde abseits der Wege einen massiven Störfaktor für Wildtiere darstellen! Eine flächige Ausgestaltung und dementsprechende Umgestaltung des Lobau-Vorlandes in eine abwechslungsreiche Wald-Wiesen-Landschaft hätte aber darüber hinaus noch den positiven Effekt, dass zusätzlicher attraktiver Lebensraum für die verschiedensten Wildtiere geschaffen würde.

Bezüglich der Maßnahmen innerhalb der Lobau ist unbedingt das Szenario „Lobau B“ zu empfehlen, das sich v.a. auf die Untere Lobau auswirkt. Durch die in diesem Szenario vorgesehene Anbindung des Hauptgewässerzuges der Unteren Lobau an die Donau, würde es zu einer deutlich erhöhten Gewässerdynamik und zur Förderung von auentypischen Standorten kommen. Der Anteil an Gewässerflächen würde sich in der Unteren Lobau erhöhen, wodurch sich der Lebensraum für den Eurasischen Biber (Castor fiber) vergrößern würde. In der Folge wäre es möglich, dass sich in der Lobau eine größere Anzahl von Bibern als derzeit vorhanden ansiedeln könnte. Durch die Anbindung wäre das Wegenetz in der Unteren Lobau tlw. nur eingeschränkt nutzbar. Manche Wege, die vorher als Rundweg genutzt werden konnten, würden nun unterbrochen sein und nur mehr Stichwege darstellen, die aus Besuchersicht nicht so attraktiv sind. Teile der Unteren Lobau würden so komplett beruhigt werden und äußerst wertvolle Wildruhezonen - v.a. auch für Reh- und Rotwild - darstellen!

\subsection{Empfehlungen aus Sicht der Vegetationsökologie}

Aus vegetationsökologischer Sicht entsprechen sowohl die Obere als auch die Untere Lobau durch die geringe Dynamik als Ausdruck der fehlenden regelmäßigen Hochwässer nur im geringen Umfang einer natürlichen Auenlandschaft. Die zunehmende Verlandung im Uferbereich - sichtbar durch den ausgedehnten Schilfgürtel als Zeichen stehender Gewässer - ist Ausdruck dieser Entwicklung. Genauso muss angemerkt werden, dass viele der nicht mehr landwirtschaftlich genutzten Offenlandflächen eine negative Entwicklung zu von Neophyten dominierten Hochstaudenfluren zeigen. Eine Ertüchtigung der „Altwässer“ und auch eine Reaktivierung ehemaliger Nebenarme durch Gewässervernetzung würden der fehlenden Gewässerdynamik entgegenwirken und Umlagerungsprozesse initialisieren.

Der aktuelle Besucherdruck bzw. auch ein zunehmender Besucherdruck kann eher als geringes Problem interpretiert werden. Das Waldbild entspricht auf Grund der hohen Persistenz eines ehemaligen intakten Waldökosystems noch immer einem naturnahen Wald (oligohemerob) - weder Waldnutzung noch BesucherInnen haben hier weiträumig sichtbare Veränderungen im Phänotyp eines Auenwaldes seit der Donauregulierung ausgelöst. Ein direkter negativer Einfluss der BesucherInnen auf den Zustand der Vegetation in den wegenahen Bereichen kann nicht erkannt werden. Weder starke Verschmutzung noch mechanische Zerstörung der Vegetation wurde nachgewiesen.

Es kann somit aus den Erkenntnissen der vorliegenden Studie abgeleitet werden, dass eine Zunahme des Besucherdruckt in keinem der untersuchten Szenarien sich negativ auf die Auenlandschaft auswirken wird. $\mathrm{Ob}$ eine stärker Nutzung als Erholungsgebiet der Lobau im Sinne der Nationalparkidee (erhalten und bewahren) zuträglich ist, kann beim momentanen Stand der Forschung nicht gesagt werden. 


\subsection{Empfehlungen aus Sicht der Gewässerökologie}

Aus gewässerökologischer Sicht ist die Entwicklung des BP Untere Lobau, der ein Teil des Nationalparks Donauauen ist, hauptsächlich durch die stetig fortschreitende Entkoppelung von der Donau geprägt, die längerfristig mit einem Verlust an aquatischen und semi-aquatischen Flächen einhergeht. Durch einen stark erhöhten Besucherdruck ist zusätzlich eine Verschlechterung der Wasserqualität in den stark frequentierten Bereichen der Oberen Lobau und vor allem den ausgewiesenen Badegewässern zu erwarten.

Die einzig wirkungsvolle Managementmaßnahme zur Erhaltung der Nationalparkqualitäten des Gebietes ist eine Wiederanbindung des ehemaligen Seitenarmsystems an die Donau (Gewässerszenario B). Die differenzierte Ausgestaltung des Lobau-Vorlandes bringt für die ökologische Funktionsfähigkeit der Gewässer nur marginale Verbesserungen.

Jegliche Maßnahme, die zur Reduktion von BesucherInnen führt, ist grundsätzlich positiv zu bewerten (wenn die Auswirkungen aus gewässerökologischer Sicht auch marginal sind). Gar nichts zu tun bedeutet jedoch aus ökologischer Sicht eine Aufgabe des Nationalparks und die Entwicklung zu einem Gebieten mit schlechter Erholungsqualität.

\subsection{Bedarf nach einem weiterführenden Biosphere Reserve Integrated Monitoring}

Im Sinne eines BRIM (The Biosphere Reserve Integrated Monitoring) sind aufgrund der städtebaulichen Entwicklungen im BP-Umfeld regelmäßige und permanente Erhebungen über die Erholungsnutzung und ihrer Auswirkungen notwendig. So sollte eine Gesamtaufnahme der Erholungsnutzung - ähnlich dem Besuchermonitoring von 1998/99 - vor dem Beginn der Besiedelung der „Seestadt Aspern“ sowie nach ihrem Bezug erfolgen. Diese Untersuchungen sollten nicht nur die Besucherzahlen erheben, sondern auch Daten zur Erholungsqualität erfassen. Insbesondere das Ausweichverhalten ist dabei zu beachteten. Dabei sollten auch die niederösterreichischen Bereiche, die an die Lobau angrenzen, berücksichtigt werden. Auch sollten die Untersuchungen feststellen, ob es Änderungen in der Struktur der Erholungssuchenden gibt (Stichwort „soziale Sukzession“, Besucherstruktur). Die Erhebungen zu den Auswirkungen der Erholungsnutzung auf den Naturraum sind weiter zu führen. Beispielsweise wären weitere Untersuchungen zur Gewässerqualität, zur Anzahl und dem Verhalten von Wildtieren und den Einflüssen auf die Vegetation entlang von Wegen erforderlich. Befragungen der neuen BewohnerInnen hinsichtlich ihres Besuchsverhaltens in der Lobau sind anzustreben, um die Prognosen dieses Projektes mit behavioralen Daten vergleichen zu können. 


\section{8 Öffentlichkeitsarbeit Stand 2012}

Publizierte Tagungsbeiträge:

- Arnberger, A., Eder, R., Taczanowska, K., Hein, T., Deussner, R., Kempter, I., Stanzer, G., Preiner, S., Reiter, K., Wagner, I. (2010): Urban sprawl impacts on protected forests: Simulating recreation behaviour using a visual discrete choice experiment. In: Findeis G., Arnberger A., Eder R., Schuster K. (Eds.), Urban People Meet Urban Forests. Conference Proceedings of the 13th European Forum on Urban Forestry, (EFUF), p. 16.

- Arnberger, A., Eder, R., Taczanowska, K. (2010): Exploring landscape preferences in the urban-rural context: A visual discrete choice experiment. In: University of Latvia, Faculty of Geography and Earth Sciences, Living in landscapes: knowledge, practice, imagination, 41; 24th PECSRL 2010, Riga, Aug 23-27, 2010]

- Taczanowska, K., Brandenburg C., Arnberger A., Tomek, H., Muhar, A. (2010): GIS as a Tool Supporting Analysis of Visitor Flows in Recreational Areas In: Gossen, M., Eilands, B., van Marwijk, R. (Eds.), Recreation and tourism in a changing world, Alterra; pp. 124-127; ISBN: 978-90-327-0387-5 [Proc. 5th Int. Conference on Monitoring and Management of Visitor Flows in Recreational and Protected Areas, Wageningen, May 30 - June 3, 2010]

- Arnberger, A., Eder, R., Taczanowska, K., Hein, T., Deussner, R., Kempter, I., Nopp-Mayr, U., Stanzer, G., Preiner, S., Reiter, K., Wagner, I. (2011): Promoting physical activities through attractive green spaces: A comparison of trail use intentions of urban green space users for the physical activities of Nordic walking, dog walking, jogging and bicycling.. In: Forestry Commission Scotland (Ed.), Urban forestry: Making the connections through green networks, 25-26 [14 Annual European Forum on Urban Forestry (EFUF), Glasgow, June 1-4, 2011]

Nicht publizierte Tagungsbeiträge:

- Arnberger, A., Eder, R., Taczanowska, K., Hein, T., Deussner, R., Kempter, I., Nopp-Mayr, U., Stanzer, G., Preiner, S., Reiter, K., Wagner, I. (2011): Simulating recreation behavior of visitors to an urban biosphere reserve using a visual discrete choice experiment. NERR 2011 (North Eastern Recreation Research Symposium), Bolton Landing, New York State, USA; 11.-13. April 2011.

- Arnberger, A., Eder, R., Taczanowska, K., Hein, T., Deussner, R., Kempter, I., Stanzer, G., Preiner, S., Reiter, K., Wagner, I. (2012): Engaging stakeholders through social science: International case studies in parks and protected areas: A case study from Vienna - The Danube Floodplains National Park. NERR 2012 (North Eastern Recreation Research Symposium), Coopers Town, New York State, USA; 31.März. -2. April 2012.

Sonstige Öffentlichkeitsarbeit:

- Gemeinsame Vorstellung der Studienergebnisse am Forschungstag im nationalparkhaus wienlobAU unter dem Titel: „Zwischen Stadtentwicklung und Erholungsdruck: Welche Perspektiven gibt es für die Lobau im Jahr 2020?“” am 18. Oktober 2011.

- Teilnahme am Wiener Forschungsfest (September 2010) mit der Präsentation des Projektes.

- Mehrere ÖIR-Newsletter, z.B Ausgaben 06/2011, 01/2012

Mehrere Artikel für wissenschaftliche Zeitschriften sind in Vorbereitung.

- Schönbrunner, I., Preiner, S., Hein, T. (submitted): Impact of drying and re-flooding of sediment on phosphorus dynamics of river-floodplain systems. Science of the Total Environment. 


\section{Literaturverzeichnis}

AICHHORN, P. and PAUKOVITS, J. (2010): Einfluss der Besucherfrequenz auf die Vegetationszusammensetzung und -struktur - Vergleich Obere und Untere Lobau, Diplomarbeit der Universität Wien.

AMMER, U. and UTSCHICK, H. (1984): Gutachten zur Waldpflegeplanung im Nationalpark Bayerischer Wald auf der Grundlage einer Ökologischen Wertanalyse, Schriftenreihe des Bayerischen Staatsministeriums f. Ernährung, Landwirtschaft und Forsten, Heft 10.

ARNBERGER, A. and EDER, R. (2009): Identifikation von Besuchersegmenten eines urbanen Erholungsgebietes anhand ihrer sozialen Wegepräferenzen. Umweltpsychologie, 13(2), 42-61.

ARNBERGER, A., AIKOH, T., EDER, R., SHOJI, Y. and MIENO, T. (2010): How many people should be in the urban forest? A comparison of trail preferences of Vienna and Sapporo forest visitor segments. Urban Forestry and Urban Greening 9, 215-225.

ARNBERGER, A., BRANDENBURG, C., CERMAK, P. and HINTERBERGER, B. (2000). Besucherstromanalyse für den Wiener Anteil am Nationalpark Donau-Auen, Bereich Lobau. Commissioned by the City of Vienna, Forest Department.

ARNBERGER, A., FREY-ROOS, F., EDER, R., MURALT, G., MOPP-MAYR, U., TOMEK, H. and ZOHMANN, M. (2009): Ökologische und soziale Tragfähigkeiten als Managementherausforderungen für suburbane Biosphärenparke am Beispiel Untere Lobau. Man \& Biosphere-Programm der Akademie der Wissenschaften, 135, Österr. Akademie der Wissenschaften, Wien; ISBN: 978-3-7001-6781-5 [http://epub.oeaw.ac.at/maboest].

BAAR, I. (2008): Endbericht OptimaLobau „Optimized management of riverine landscapes based on a multi-criteria Decision Support System - Macrophytes.

BASSI, M. (2003): Habitat-Modelle in der Wildökologie. Dipl.-Arb., Universität für Bodenkultur Wien, $109 \mathrm{~S}$.

BLUME, H.-P. \& SUKOPP, H. (1976): Ökologische Bedeutung anthropogener Bodenveränderungen. Schriftenreihe Vegetationskunde10: 7 - 89. Bonn, Bad Godesberg, nach GRABHERR, G., KOCH, G.; KIRCHMEIR, H. \& REITER, K. (1998): Hemerobie österreichischer Waldökosysteme: Veröffentlichungen des Österreichischen MaB-Programms; Band 17. Universitätsverlag Wagner, Innsbruck. $493 \mathrm{~S}$.

BORNKAMM, R. (1980): Hemerobie und Landschaftsplanung; Landschaft und Stadt. H.12:49-55.

BRANDENBURG, C. (2001): Erfassung und Modellierung von Besuchsfrequenzen in Erholungs- und Schutzgebieten - Anwendungsbeispiel Nationalpark Donau-Auen, Teilgebiet Lobau. Institut für Freiraumgestaltung und Landschaftspflege. Universität für Bodenkultur, Wien. Dissertation.

BRAUN- BLANQUET, J. (1964): Pflanzensoziologie. Grundzüge der Vegetationskunde. Springer Verlag, Wien New York.

CAVENS, D., GLOOR, C., NAGEL, K., LANGE, E. and SCHMID, W. A. (2004): A Framework for Integrating Visual Quality Modelling within an Agent-Based Hiking Simulation for the Swiss Alps. Working Papers of the Finnish Forest Research Institute 2.

DEBELJAK, M., DZEROSKI, S., KLEMEN, J., KOBLER, A. and ADAMIC, M. (2001): Habitat suitability modelling for red deer (Cervus elaphus L.) in South-central Slovenia with classification trees. Ecological Modelling 138, 321-330.

DIERSCHKE, H. (1994): Pflanzensoziologie: Grundlagen und Methoden; 55 Tabellen. Eugen GmbH \& Co, Stuttgart. $683 \mathrm{~S}$.

ENZENHOFER K., MAYRHOFER S. and REITER K. (2009): Hemerobie der Wälder im Biosphärenpark Wienerwald. Verhandlungen der Zoologisch-Botanischen Gesellschat Österreich 146: $1-16$. 
FRANKLIN, S. and GRAESSER, A. (1997): Is it an Agent, or Just a Program? A Taxonomy for Autonomous Agents. In: Müller, J. P., Wooldridge, M. J. and Jennings, N. R. (Ed.): Intelligent Agents III: Agent Theories, Architectures, and Languages. ECAI'96 Workshop Proceedings, Springer, Berlin et al. 21-35.

FUCHS, E., GIEBEL, H., HETTRICH, A., HÜSING, V., ROSENZWEIG, S. and THEIS, H.J. (2003): Einsatz von ökologischen Modellen in der Wasser- und Schifffahrtsverwaltung - Das integrierte Flussbaumodell INFORM 2.0, BfG-Mitteilungen Nr. 25, ISSN-Nr. 1431-2409, 212 S.

GIMBLETT, H. R., DURNOTA, B. and ITAMI, B. (1996): Spatially-Explicit Autonomous Agents for Modelling Recreation Use in Complex Wilderness Landscapes. Complexity International 3.

GIMBLETT, H. R. (Ed.) (2002): Integrating Geographic Information Systems and Agent-Based Modeling Techniques for Simulating Social and Ecological Proccesses. Oxford University Press, New York.

GIMBLETT, H.R., RICHARDS, M.T. and ITAMI, R.M. (2002): Simulating Wildland Recreation Use and Conflicting Spatial Interactions using Rule-Driven Intelligent Agents, in Gimblett, H.R. (ed.): Integrating Geographic Information Systems and Agent-Based Modelling Techniques for Simulating Social and Ecological Processes, Oxford University Press, Oxford, pp. 211-243.

GLAVAC, V. (1996): Vegetationsökologie: Grundfragen, Aufgaben, Methoden; 36Tabellen. Gustav Fischer Verlag Jena / Stuttgart / Lübeck / Ulm. 358 S.

GLOOR, C., CAVENS, D., LANGE, E., NAGEL, K. and SCHMID, W. (2003): A Pedestran Simulation for Very Large Scale Applications. Klagenfurter Geographische Schriften. Institut für Geographie und Regionalforschung der Universität Klagenfurt. Heft 23: 167-187.

GRABHERR, G., KOCH, G.; KIRCHMEIR, H. and REITER, K. (1998): Hemerobie österreichischer Waldökosysteme: Veröffentlichungen des Österreichischen MaB-Programms; Band 17. Universitätsverlag Wagner, Innsbruck. $493 \mathrm{~S}$.

GRASS, V. (1993): Salicetea purpureae; in: Grabherr G. et al. (Hrsg.): Die Pflanzengesellschaften Österreichs. G.Fischer Verlag Jena.

HARTMAN, G. and TÖRNLÖV, S. (2006): Influence of watercourse depth and width on dambuilding behaviour by Eurasian beaver (Castor fiber). Journal of Zoology 268, 127-131.

HENSHER, D.A., ROSE, J.M. and GREENE, W.H. (2005): Applied Choice Analysis: A Primer. Cambridge: Univ. Press.

HETTRICH A. and ROSENZWEIG S. (2002): Einsatz multivariater statistischer Modelle zur Ermittlung der Zusammenhänge zwischen Biotik und Abiotik sowie Prognose des ökologischen Zustandes von Flussauen. - I: Hydrologie und Wasserbewirtschaftung 46, H. 4, S. 156-165.

HILL, M.O. (1979): Twinspan - A Fortran program for arranging multivariate data in an ordererd two-way-table by classification of the individuals and attributes. Cornell University, Ithaca, USA.

HÜBL, E. (1972): Zur biologischen und pflanzengeographischen Charakteristik der Auwälder Wiens. In: Naturgeschichte Wiens, Band 2. Jugend und Volk Verlagsgesellschaft m. b. H., Wien-München. S. $707-716$.

ILLEDITS, A., ZSAK, K. (2012): Hemerobiebewertung der Waldökosysteme im Wiener Anteil des Nationalpark Donau-Auen; Diplomarbeit Universität Wien.

ITAMI, R.M. (2002): 'Mobile Agents with Spatial Intelligence', in Gimblett, H.R. (ed.) Integrating Geographical Information Systems and Agent-Based technologies for modelling and simulating social and ecological phenomena, Oxford University Press, Oxford, pp. 191-210.

JALAS J. (1955): Hemerobe und hemerochore Pflanzenarten. Ein terminologischer Versuch. - Acta Soc. Fauna Flora Fenn.. 72 (11): 1-15.

JANAUER, G. A. and E. Heindl (1998): Die Schätzskala nach Kohler: Zur Gültigkeit der Funktion $\mathrm{f}(\mathrm{y})=\mathrm{ax}^{3}$ als Maß für die Pflanzenmenge von Makrophyten. Verh.Zool-Bot.Ges.Österreich 135: 117128. 
JENSEN, P., JEPPESEN, E., OLRIK, K. and KRISTENSEN, P. (1994): Impact of nutrients and physical factors on the shift from cyanobacterial to chlorophyte dominance in shallow Danish lakes. Canadian Journal of Fisheries and Aquatic Sciences, vol. 51, p 1692-1699.

JOCHEM, R. and VAN DER GREFT, J. (2005): MASOOR. Multi-Agent Simulation of Outdoor Recreation. Alterra, Wageningen University and Research Centre, Wageningen (unpublished report).

JOCHEM, R., MARWIJK, R. v., POUWELS, R. and PITT, D. G. (2008): MASOOR: modeling the transaction of people and environment on dense trail networks in natural resource settings. In: Gimblett, R. and Skov-Petersen, H. (Ed.): Monitoring, Simulation and Management of Visitor Landscapes. University of Arizona Press, Tucson, USA. 269-294; [http://www.uapress.arizona.edu/onlinebks/Monitoring_Visitor_Landscapes.pdf].

KEMPERMAN, A. D. A. M. and TIMMERMANS, H. J. P. (2006): Heterogeneity in urban park use of aging visitors: a latent class analysis. Leisure Sciences, 28: 57-71.

KOHLER, A. and G. A. JANAUER (1995): Zur Methodik der Untersuchung von aquatischen Makrophyten in Fließgewässer. In: Steinberg Bernhard Klapper- Handbuch Angewandte Limnologie (Landsberg/Lech): 1-22.

KOWARIK, I. (1988): Zum menschlichen Einfluß auf Flora und Vegetation. Theoretische Konzepte und ein Quantifizierungsansatz am Beispiel von Berlin (West). Landschaftsentwicklung und Umweltforschung 56, $280 \mathrm{~S}$.

LAZOWSKI, W. (1984): Über die Auwälder an Donau, March und Thaya. ÖKO-L 6/4. Biologiezentrum Linz.

LOUVIERE, J. J., HENSHER, D. A. and SWAIT, J. D. (2000): Stated choice methods - Analysis and application. Cambridge, UK: University Press.

MARGL, H. (1973): Pflanzengesellschaften und ihre standortgebundene Verbreitung in teilweise abgedämmten Donauauen (Untere Lobau). Zool.-Bot. Ges. Österreich. 50 S.

MARINGER, A. and SLOTTA-BACHMAYR, L. (2006): A GIS-based habitat-suitability model as a tool for the management of beavers Castor fiber. Acta Theriologica 51(4), 373-382.

MARWIJK, R. v. (2009): These routes are made for walking: understanding the transactions between nature, recreational behaviour and environmental meanings in Dwingelderveld National Park, the Netherlands. Wageningen University. Dissertation; [http://www.groenendestad.nl/upload/publicaties/documenten/Dissertation_VanMarwijk.pdf].

MAYER, M. (2007): Validierung eines Vegetationsmodells durch Vegetationsaufnahmen einer repräsentativen Stichprobe der Unteren Lobau, Diplomarbeit der Universität Wien.

MINISTERIUM für Umwelt, Naturschutz und, Raumordnung des Landes Brandenburg (MUNR) (Hrsg.) (1999): Artenschutzprogramm Elbebiber und Fischotter. Potsdam.

MÜHLENBERG, M. (1989): Freilandökologie; Heidelberg, Wiesbaden; Verlag Quelle \& Meyer; Heidelberg, Wiesbaden; $430 \mathrm{~S}$.

MÜLLER-SCHWARZE, D. and SUN, L. (2003): The Beaver - Natural History of a Wetland Engineer. Cornell University Press, New York.

PARTL, E. (2001): Wechselwirkungen zwischen Wald als Habitat und Rehwild als Standortfaktor bei der Waldsanierung. Diss., Universität für Bodenkultur Wien, $221 \mathrm{~S}$.

RAHM, U., BAETTIG, M. (1996): Der Biber in der Schweiz. Bestand, Gefährdung, Schutz. Schriftenreihe Umwelt Nr. 249, BUWAL (Bundesamt für Umwelt, Wald und Landschaft), Bern, 68 S.

REICHHART, T. and ARNBERGER, A. (2010): Exploring the influence of speed, social, managerial and physical factors on shared trail preferences using a $3 \mathrm{D}$ computer animated choice experiment. Landscape and Urban Planning 96(1), 1-11. 
REIMOSER, F. (1986): Wechselwirkungen zwischen Waldstruktur, Rehwildverteilung und Rehwildbejagbarkeit in Abhängigleit von der waldbaulichen Betriebsform. Diss., Universität für Bodenkultut Wien, VWGÖ-Verlag, Wien, Bd. 28, 319 S.

ROSENAU, S. (2003): "Bibermanagementplan" - Entwicklung eines Schutzkonzeptes für den Biber (Castor fiber L.) im Bereich der Berliner Havel. Zwischenbericht Juni 2003, unveröffentlicht.

ROTTER, D. (1999): Die Verlandungsdynamik der Donauauen südöstlich von Wien.Geobotanik und Ökologie der Donaualtwässer bei Wien (Schratt - Ehrendorfer, L. \& Rotter, D. 1999):163-207. Oberösterreichisches Landesmuseum, Biologiezentrum Linz.

SAUBERER, A. (1942): Vegetationsverhältnisse der Unteren Lobau. Niederdonau/Natur und Kultur. 17 Heft. Verlag Karl Kühne, Wien-Leipzig.

SCARPA, R. and THIENE, M. (2005): Destination choice models for rock climbing in the Northeastern Alps: a latent-class approach based on intensity of preferences. Land Economics, 81(3): 426-444.

SCHRATT-EHRENDORFER, L. (2011): Donau und Auenlandschaft, Ein Lebensraum voller Gegensätze. In: Ökosystem Wien: Die Naturgeschichte einer Stadt. Böhlau Verlag, Wien-KölnWeimar. S. 331-391.

SCHROM-FEIERTAG, MUHAR, A., ALMER, A., TACZANOWSKA, K., BRANDENBURG, Ch., BAUER, D., RAY, M., LULEY, P., STEZL, H., HAUBENBERGER, G., BRUNNER, R., TOMEK, H. und SCHECHTNER, K., (2009): Nachhaltiges Lenkungs- und Informationssystem für Besucher und Betreiber von Nationalparks auf der Basis eines mobilen Guides und dynamischer Auslastungsprognosen. BMVIT, FFG, Österreichsiche Weltraumforschung, 133.

SCHULZ, L. (1981): Nährstoffeintrag in Seen durch Badegäste.- Zbl. Bakt. Hyg,. I. Abt. Orig. B 173, S. $528-548$.

SCHWAB, G. (2002): Biber in der Kulturlandschaft: Konflikte und Lösungen. Handbuch für Biberatur. Haus im Moos, Karlshuld-Kleinhohenried, $11 \mathrm{~S}$.

SKOV-PETERSEN (2009): Kvintus Model. GIS and Agent-based Modelling Blog [http://gisagents.blogspot.com/2009/01/kvintus-model.html].

STADTENTWICKLUNG WIEN, MA 18 (2005): Stadtentwicklungsplan 2005. Download unter: http://www.wien.gv.at/stadtentwicklung/strategien/step/step05/download/pdf/step-gesamt.pdf

SUKOPP, H. (1969): Der Einfluß des Menschen auf die Vegetation. Vegetatio 17: 360 - 371, nach GRABHERR, G., KOCH, G., KIRCHMEIR, H. \& REITER, K. (1998): Hemerobie österreichischer Waldökosysteme: Veröffentlichungen des Österreichischen MaB-Programms; Band 17. Universitätsverlag Wagner, Innsbruck. S. $56-62$.

SUKOPP, H. (1972): Wandel von Flora und Vegetation in Mitteleuropa unter dem Einfluß des Menschen. - Berichte über Landwirtschaft, Bd. 50: 112-139, , nach GRABHERR, G., KOCH, G.; KIRCHMEIR, H. \& REITER, K. (1998): Hemerobie österreichischer Waldökosysteme: Veröffentlichungen des Österreichischen MaB-Programms; Band 17. Universitätsverlag Wagner, Innsbruck. S. $56-62$.

SUKOPP, H. (1976): Dynamik und Konstanz in der Flora der Bundesrepublik Deutschland. Schr. R. Vegetationskkde 10: 9 - 27, nach SUKOPP, H. \& WITTIG, R. (Hrsg.) mit Beiträgen von BLUME, H.- P. (1998): Stadtökologie. 2., überarbeitete und ergänzte Auflage. Gustav Fischer Verlag, Stuttgart / Jena / Lübeck / Ulm. S. $256-257$.

SUKOPP, H., KUNICK, W., RUNGE, M., ZACHARIAS, F. (1974): Ökologische Charakteristik von Großstädten, dargestellt am Beispiel Berlins. Verh. Ges. Ökologie 1973, Saarbrücken, Junk, Den Haag: $383-403$.

TACZANOWSKA K. (2009): Modelling the Spatial Distribution of Visitors in Recreational Areas. Dissertation, 199; Universität für Bodenkultur Wien; [https://zidapps.boku.ac.at/ abstracts/oe_list.php?paID $=3 \&$ paSID $=7284 \& p a S F=-1 \& p a C F=0 \& p a L I S T=0 \&$ language_id=DE]. 
TACZANOWSKA, K., ARNBERGER, A. and MUHAR, A. (2008a): Exploring Spatial Behavior of Individual Visitors as Background for Agent-Based Simulation. In: Gimblett, R. and Skov-Petersen, H. (Ed.): Monitoring, Simulation and Management of Visitor Landscapes. University of Arizona Press, Tucson, USA. 159-174; [http://www.uapress.arizona.edu/onlinebks/Monitoring Visitor_Landscapes.pdf].

TACZANOWSKA, K., MUHAR, A. and BRANDENBURG, C. (2008b): Potential and limitations of GPS tracking for monitoring spatial and temporal aspects of visior behaviour in rereational areas. In: Raschi, A. and Trampetti, S. (Ed.): The Fourth International Conference on Monitoring and Management of Visitor Flows in Recreational and Protected Areas, Montecatini Terme, Italy, 14.-19. Oct. 2008. 451-456.

TÜXEN, R. (1956): Die heutige potentielle natürliche Vegetation als Gegenstand der Vegetationskartierung. In: TÜXEN R., 1956: Angewandte Pflanzensoziologie. 13. Bundesanstalt für Vegetationskartierung, Stolzenau, Weser. $55 \mathrm{~S}$.

VERMUNT, J. K. and MAGIDSON, J. (2003): Latent Gold Choice User's Manual, Belmont, MA: Statistical Innovations, Inc.

WENDELBERGER, G. (1975): Ökosystem Auwald, Bundesministerium für Wissenschaft und Forschung, Österreich. 54 S.

WILD, A. (1995): Umweltorientierte Bodenkunde. Spektrum Akademischer Verlag Heidelberg, Berlin, Oxford.

WOOLDRIDGE, M. J. (2005): An introduction to multiagent systems. Wiley, Chichester.

ZSUFFA, I.J. (2000): Multi-criteria decision support or the revitalisation of river floodplains. Doctoral thesis, Wageningen University. 


\section{Verzeichnisse}

\subsection{Abbildungsverzeichnis}

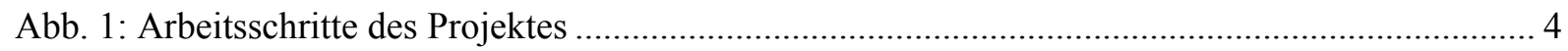

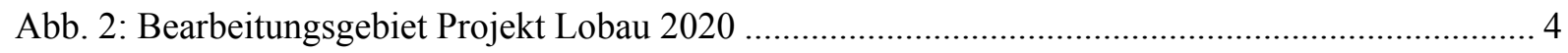

Abb. 3: 3D-Darstellung der geplanten Bebauung am Flugfeld Aspern. Quelle: Masterplan Flugfeld

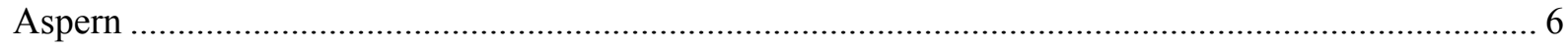

Abb. 4: Strukturdaten im und um das Flugfeld Aspern. Quelle: ÖIR, 2006. Sekundärnetz Flugfeld

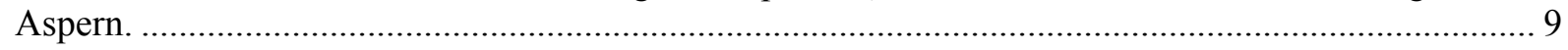

Abb. 5: Darstellung von Grundszenario „Offen $1 \mathrm{~b}$ verlandet“ aus dem Projekt Optima Lobau. ......... 13

Abb. 6: Hauptstraßennetz B in Wien inkl. Bundesstraßen A und S. Quelle: MA 18 ........................... 15

Abb. 7: Stellplatzerhebung Lobaueingänge. Anzahl der abgestellten PKW (11.00 - 12.30). Quelle:

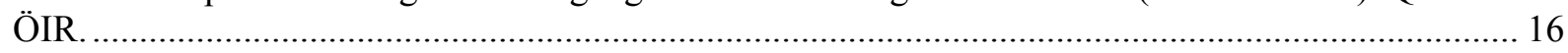

Abb. 8: Öffentliche Verkehrsanbindung Flugfeld Aspern. Quelle: ÖIR, 2006. Sekundärnetz Flugfeld.

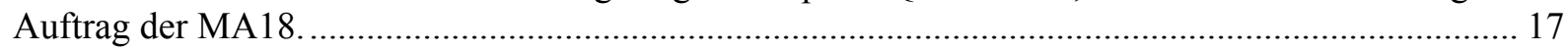

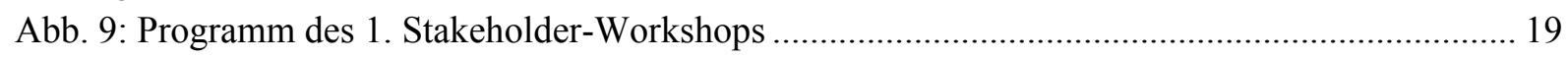

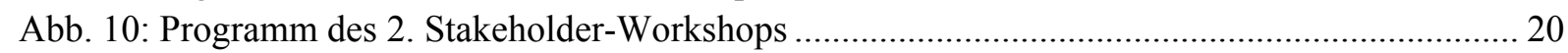

Abb. 11: Radwegkarte mit Vorschlägen zu Radwegführungen im Lobau-Vorland basierend auf dem 2.

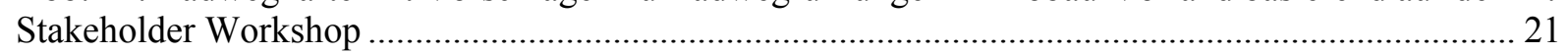

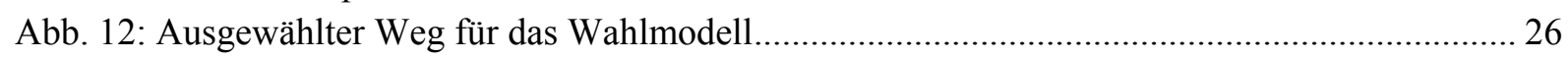

Abb. 13: Fotoserien zur Größenskalierung der Personen in den Bildern ............................................. 27

Abb. 14: Beispiel für ein Wahlset mit vier Szenarien aus dem Wahlmodell ........................................ 27

Abb. 15: Beispiel für ein Wahlset mit vier Szenarien aus dem Wahlmodell ....................................... 28

Abb. 16: Anzahl der Befragten pro Bezirk/Gemeinde .................................................................. 29

Abb. 17: Lage der Wohnorte der Befragten im 22. Bezirk und der Gemeinde Groß-Enzersdorf......... 29

Abb. 18: Verteilung der Befragten nach Anreisearten (Eingänge Saltenstrasse und Esslingerfurt) ..... 30

Abb. 19: Verteilung der Befragten nach Anreisearten - Vergleich der Eingänge Saltenstrasse und

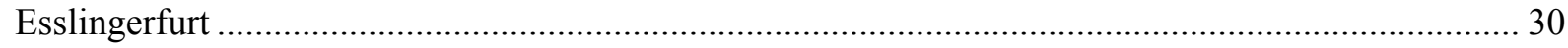

Abb. 20: Erreichbarkeit der NP-Eingänge Saltenstrasse und Esslingerfurt (Graustufen). Darstellung der Quell-Ziel-Beziehungen (Wohnort-Eingang) für die Eingänge Saltenstrasse und Esslingerfurt .... 31 Abb. 21: Erreichbarkeit der NP-Eingänge Saltenstrasse und Esslingerfurt (Graustufen). Darstellung der Quell-Ziel-Beziehungen (Wohnort-Eingang) gruppiert nach Anreiseart .

Abb. 22: Präferenzgrad für die im Wahlmodell dargestellten Landschaftstypen ( $\mathrm{N}=520)$. Je positiver der Wert, desto höher der Präferenzgrad......................................................................................... 32 Abb. 23: Präferenzgrad für die im Wahlmodell dargestellte Besucheranzahl $(\mathrm{N}=520)$. Je positiver der

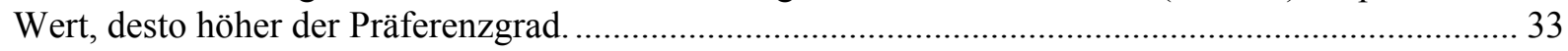
Abb. 24: Präferenzgrad für den im Wahlmodell dargestellten Gewässertypus $(\mathrm{N}=520)$. Je positiver der

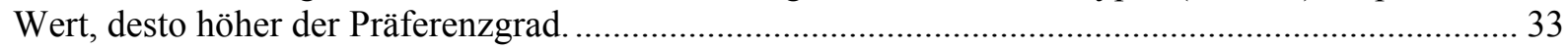

Abb. 25: Komponente eines MASOOR-Models (Jochem et al. 2008) …............................................ 35

Abb. 26: Bewegungsphasen eines MASOOR-Agenten: 1) Entry Phase - in der Bewegungsphase werden bestimmte Attraktionen / Zielpunkte besucht; diese Phase ist jedoch optional, manche Agententypen fangen den Besuch eines Erholungsgebietes direkt in der zweiten Bewegungsphase an, ohne konkrete Ziele besucht zu haben; 2) Browse Phase - Bewegung ohne vordefinierte Ziele, lokale Entscheidungen auf Basis vordefinierter Bewegungskriterien. Die Phase dauert solange, bis $75 \%$ der geplanten Besuchszeit erreicht werden; 3) Directed Phase - der Agent orientiert sich langsam Richtung Ausgang; 4) Exit Phase - die letzte Bewegungsphase (die letzten $10 \%$ der vordefinierten Zeit), die zum Verlassen des Erholungsgebietes führt.

Abb. 27: Inputdaten für die Simulation der Besucherströme in der Lobau. A) Wegenetz: Variante „Lobau A“ und 12 Eingänge; B) Wegenetz: Variante „Lobau B“ und 12 Eingänge 
Abb. 28: Inputdaten für die Simulation der Besucherströme in der Lobau. A) Wegenetz: Variante „Lobau A + Vorland A“ und 11 Eingänge; B) Wegenetz: Variante „Lobau B + Vorland A“ und 11 Eingänge

Abb. 29: Inputdaten für die Simulation der Besucherströme in der Lobau. A) Wegenetz: Variante „Lobau A + Vorland A“ und 11 Eingänge; B) Wegenetz: Variante „Lobau B + Vorland A“ und 11 Eingänge

Abb. 30: Thematische Eigenschaften des Lobau-Wegenetzes: Wegbreite …....................................... 42

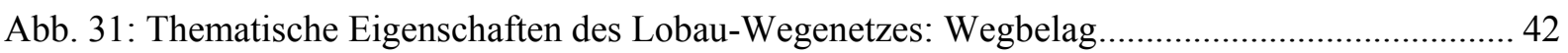

Abb. 32: Thematische Eigenschaften des Lobau-Wegenetzes: Wegbegleitbepflanzung........................ 43

Abb. 33: Thematische Eigenschaften des Lobau-Wegenetzes: Landschaftstypus ................................. 43

Abb. 34: Thematische Eigenschaften des Lobau-Wegenetzes: Hintergrund / Horizont ........................ 44

Abb. 35: Thematische Eigenschaften des Lobau-Wegenetzes: Ausblick auf Gewässer ....................... 44

Abb. 36: Thematische Eigenschaften des Lobau-Wegenetzes: Infrastruktur....................................... 45

Abb. 37: Thematische Eigenschaften des Wegenetzes im Bereich des Lobau-Vorlands: Wegbreite... 46

Abb. 38: Thematische Eigenschaften des Wegenetzes im Bereich des Lobau-Vorlands: Wegbelag ... 46

Abb. 39: Thematische Eigenschaften des Wegenetzes im Bereich des Lobau-Vorlands:

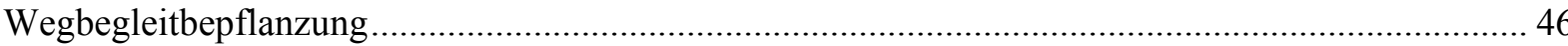

Abb. 40: Thematische Eigenschaften des Wegenetzes im Bereich des Lobau-Vorlands:

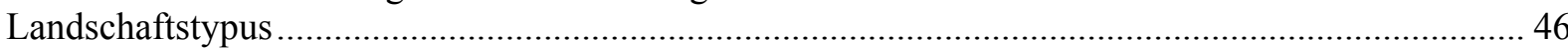

Abb. 41: Thematische Eigenschaften des Wegenetzes im Bereich des Lobau-Vorlands: Hintergrund /

Horizont.

Abb. 42: Thematische Eigenschaften des Wegenetzes im Bereich des Lobau-Vorlands: Ausblick auf

Gewässer....

Abb. 43: Thematische Eigenschaften des Wegenetzes im Bereich des Lobau-Vorlands: Infrastruktur 47

Abb. 44: Bewegungsphasen eines MASOOR-Agenten .....

Abb. 45: Berechnung des neuen Wegtyp-Attributs. Zusammenstellung mehrerer Eigenschaften in eine kategorische Variable.

Abb. 46: Die relativen Wegtyppräferenzen der Agenten (Typ 01 - Fußgeher) für verschiedene Managementszenarien innerhalb der Lobau (Variante A und B) und im Bereich des Lobau-Vorlands (Variante A und B). Es ist ersichtlich, dass die FußgängerInnen die flächige Ausgestaltungsvariante des Vorlandes (Wald und Wiesen) bevorzugen.

Abb. 47: Die relativen Wegtyppräferenzen der Agenten (Typ 02 - Hundebesitzer) für verschiedene Managementszenarien innerhalb der Lobau (Variante A und B) und im Bereich des Lobau-Vorlands (Variante A und B).

Abb. 48: Die relativen Wegtyppräferenzen der Agenten (Typ 03-Nordic WalkerInnen) für verschiedene Managementszenarien innerhalb der Lobau (Variante A und B) und im Bereich des Lobau-Vorlands (Variante A und B).

Abb. 49: Die relativen Wegtyppräferenzen der Agenten (Typ 04 - JoggerInnen) für verschiedene Managementszenarien innerhalb der Lobau (Variante A und B) und im Bereich des Lobau-Vorlands (Variante A und B).

Abb. 50: Die relativen Wegtyppräferenzen der Agenten (Typ 05-RadfahrerInnen A) für verschiedene Managementszenarien innerhalb der Lobau (Variante A und B) und im Bereich des Lobau-Vorlands (Variante A und B).

Abb. 51: Die relativen Wegtyppräferenzen der Agenten (Typ 06 - RadfahrerInnen B) für verschiedene Managementszenarien innerhalb der Lobau (Variante A und B) und im Bereich des Lobau-Vorlands (Variante A und B).

Abb. 52: Ergebnisse der Erreichbarkeitsanalyse für die Haupteingänge in die Lobau unter besonderer Berücksichtigung der Entfernungszone 2-4 km. (Datenbasis: Befragung „Perspektive LOBAU 2020“); Unterschiedliche Farben der Punktdaten ermöglichen eine Zuordnung der Wohnorte und der, von den BesucherInnen, benutzten Eingänge. 
Abb. 53: Ergebnisse der Erreichbarkeitsanalyse für die Lobau-Haupteingänge unter besonderer Berücksichtigung der Entfernungszone 2-4 km. Wahl der Eingänge für die BesucherInnen aus Wien sowie aus der Gemeinde Gross-Enzersdorf..

Abb. 54: Vergleich der beobachteten Besucherzahlen (1999) und der Prognose für das Jahr 2025 (kartographische Darstellung); A) Besucherzahlen an einem Spitzentag; B) Besucherzahlen an einem Werktag.....

Abb. 55: Vergleich der beobachteten Besucherzahlen (1999) und der Prognose für das Jahr 2025 (Balkendiagramm). Inputdaten für die MASOOR Simulation. A) Besucherzahlen an einem Spitzentag;

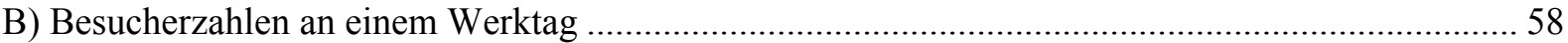

Abb. 56: Ergebnisse der MASOOR Simulation. Räumliche Verteilung der BesucherInnen in der Lobau an einem Spitzenbesuchstag für das Jahr 1999 - Szenario-Nr. 01 (Bestand). 60

Abb. 57: Ergebnisse der MASOOR Simulation. Räumliche Verteilung der BesucherInnen in der Lobau an einem Spitzenbesuchstag für das Jahr 2025 - Szenario-Nr. 03 („Laissez-faire -Szenario“; keine Maßnahmen getroffen)

Abb. 58: Ergebnisse der MASOOR Simulation. Räumliche Verteilung der BesucherInnen in der Lobau an einem Spitzenbesuchstag für das Jahr 2025 - Szenario-Nr. 05 (Gewässerszenario 1b, sonst keine Maßnahmen getroffen).

Abb. 59: Ergebnisse der MASOOR Simulation. Räumliche Verteilung der BesucherInnen in der Lobau an einem Spitzenbesuchstag für das Jahr 2025 - Szenario-Nr. 07 (lineare Ausgestaltung des Lobau-Vorlandes mit neuen Radwegen mit Alleen, Parkraumbewirtschaftung, kein Gewässerszenario 1b).

Abb. 60: Ergebnisse der MASOOR Simulation. Räumliche Verteilung der BesucherInnen in der Lobau an einem Spitzenbesuchstag für das Jahr 2025 - Szenario-Nr. 09 (flächige Ausgestaltung des Lobau-Vorlandes mit neuen Radwegen mit Alleen, Parkraumbewirtschaftung, kein Gewässerszenario 1b).

Abb. 61: Ergebnisse der MASOOR Simulation. Räumliche Verteilung der BesucherInnen in der Lobau an einem Spitzenbesuchstag für das Jahr 2025 - Szenario-Nr. 11 (lineare Ausgestaltung des Lobau-Vorlandes mit neuen Radwegen mit Alleen, Parkraumbewirtschaftung, Gewässerszenario 1b).

Abb. 62: Ergebnisse der MASOOR Simulation. Räumliche Verteilung der BesucherInnen in der Lobau an einem Spitzenbesuchstag für das Jahr 2025 - Szenario-Nr. 13 (flächige Ausgestaltung des Lobau-Vorlandes mit neuen Radwegen mit Alleen, Parkraumbewirtschaftung, Gewässerszenario 1b).

Abb. 63: Ergebnisse der MASOOR Simulation. Räumliche Verteilung der BesucherInnen in der Lobau an einem durchschnittlichen Werktag für das Jahr 1999 - Szenario-Nr. 02 (Bestand)

Abb. 64: Ergebnisse der MASOOR Simulation. Räumliche Verteilung der BesucherInnen in der Lobau an einem durchschnittlichen Werktag für das Jahr 2025 - Szenario-Nr. 04 (,Laissez-faire Szenario“; keine Maßnahmen getroffen).

Abb. 65: Ergebnisse der MASOOR Simulation. Räumliche Verteilung der BesucherInnen in der Lobau an einem durchschnittlichen Werktag für das Jahr 2025 - Szenario-Nr. 06 (Gewässerszenario $1 \mathrm{~b}$, sonst keine Maßnahmen).

Abb. 66: Ergebnisse der MASOOR Simulation. Räumliche Verteilung der BesucherInnen in der Lobau an einem durchschnittlichen Werktag für das Jahr 2025 - Szenario-Nr. 08 (lineare Ausgestaltung des Lobau-Vorlandes mit neuen Radwegen mit Alleen, Parkraumbewirtschaftung, kein Gewässerszenario $1 b)$.

Abb. 67: Ergebnisse der MASOOR Simulation. Räumliche Verteilung der BesucherInnen in der Lobau an einem durchschnittlichen Werktag für das Jahr 2025 - Szenario-Nr. 10 (flächige Ausgestaltung des Lobau-Vorlandes mit neuen Radwegen mit Alleen, Parkraumbewirtschaftung, kein Gewässerszenario $1 b)$.

Abb. 68: Ergebnisse der MASOOR Simulation. Räumliche Verteilung der BesucherInnen in der Lobau an einem durchschnittlichen Werktag für das Jahr 2025 - Szenario-Nr. 12 (lineare Ausgestaltung des Lobau-Vorlandes mit neuen Radwegen mit Alleen, Parkraumbewirtschaftung, Gewässerszenario 1b). 
Abb. 69: Ergebnisse der MASOOR Simulation. Räumliche Verteilung der BesucherInnen in der Lobau an einem durchschnittlichen Werktag für das Jahr 2025 - Szenario-Nr. 14 (flächige Ausgestaltung des Lobau-Vorlandes mit neuen Radwegen mit Alleen, Parkraumbewirtschaftung, Gewässerszenario $1 b)$.

Abb. 70: Modellierte Vegetationskarte auf Basis der Bewertung der Forstkartierung der Gemeinde

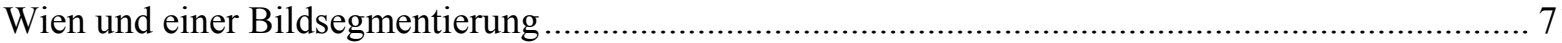

Abb. 71: Karte der Potentiell Natürlichen Vegetation im Gebiet der Lobau ........................................ 74

Abb. 72: Karte der Verteilung der Probepunkte zur Untersuchung der Hemerobie .............................. 75

Abb. 73: Verknüpfungsbaum der Hemerobiebewertung (aus Enzenhofer et al., 2009)........................ 76

Abb. 74: Karte der Hemerobie der Waldökosysteme der Lobau - (aus Illedits \& Zsak, 2012)

Abb. 75: Karte der Hemerobie der Lobau - kompiliert aus der Karte der Hemerobie - Waldökosystem und der gutachterlichen Zuweisung von Hemerobiewerte bei Offenland Vegetationstypen und Vorwaldstadien.

Abb. 76: Karte der Sensibilität der Ökosysteme in der Lobau - abgeleitet aus der Karte zur Hemerobie.

Abb. 77: Karte der Sensibilität der Ökosysteme in der Lobau - abgeleitet aus der Karte zur Hemerobie.

Abb. 78: Karten der klassifizierten Besucherfrequenz an einem normalen Arbeitstag durch Hot Spot Analysis der Besucherfrequenz und einer Verteilung auf drei Klassen durch Equal Interval; Karte a: Normaler Arbeitstag 1999; Karte b: Normaler Arbeitstag 2012; Karte c: Normaler Arbeitstag unter Einbeziehung des Gewässerszenarios 1b; Karte d: Normaler Arbeitstag unter Einbeziehung des Gewässerszenarios $1 b$ und einer flächigen Ausprägung der Vorlandszenarien

Abb. 79: Gegenüberstellung Besucherfrequenzen (Klassen 1 = geringe Besucherfrequenz, Klasse 2 = mittlere Besuchfrequenz, Klasse 3 = hohe Besucherfrequenz) und Vegetationssensibilität (gering bis hoch) durch Vergleiche der Streckenlänge der Wegabschnitte; Grafik a: Werktag 1999; Grafik b: Werktag 2012; Grafik c: Werktag unter Einbeziehung des Gewässerszenarios 1b; Grafik d: Werktag unter Einbeziehung des Gewässerszenarios $1 b$ und einer flächigen Ausprägung der Vorlandszenarien.

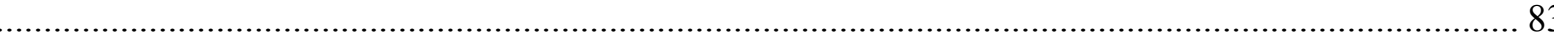

Abb. 80: Darstellung der Kombination einer modellierten Vegetationskarte und der Biotoptypenkarte

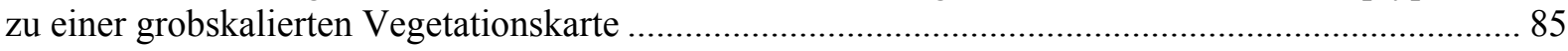

Abb. 81: Stichprobenplan der Freilanderhebungen im Sommer 2009 mit 213 ausgewiesenen

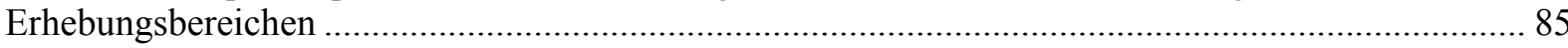

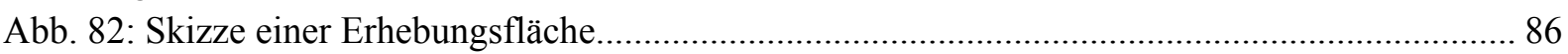

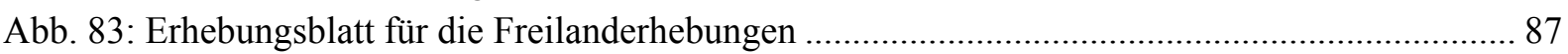

Abb. 84: Prozentuelle Anteile der aufgenommenen Wegetypen der Oberen Lobau ................................ 89

Abb. 85: Prozentuelle Anteile der aufgenommenen Wegetypen der Unteren Lobau ............................ 89

Abb. 86: Auftretende Disturbance in der Oberen und Unteren Lobau.............................................. 90

Abb. 87: Zusammenhang und Wirkung der Hauptfaktoren auf die Makrophytenverteilung (Baart, 2008).

Abb. 88: Berechnung der Abweichung von einem Chl-a-Schwellenwert von $10 \mu \mathrm{g} 1-1$ in wenig durchströmten Gewässerabschnitten in \%.

Abb. 89: Modellierte Werte der Makrophytendeckung (aufsummierte, umgerechnete KohlerSchätzungen) für das Gewässerszenario A mit den Besucherfrequenzen von 1999 und 2025 (Baart, 2008 adaptiert).

Abb. 90: Modellierte Werte der Makrophytendeckung (aufsummierte, umgerechnete KohlerSchätzungen) für das Gewässerszenario B mit den Besucherfrequenzen von 2025 (Baart, 2008 adaptiert)......

Abb. 91: Modellierte Werte des mittleren jährlichen Chlorophyll-a-Gehaltes in der Unteren Lobau Gewässerszenario A - 1999.

Abb. 92: Modellierte Werte des mittleren jährlichen Chlorophyll-a-Gehaltes in der Unteren Lobau Gewässerszenario A - 2025. 
Abb. 93: Modellierte Werte des mittleren jährlichen Chlorophyll-a-Gehaltes in der Unteren Lobau -

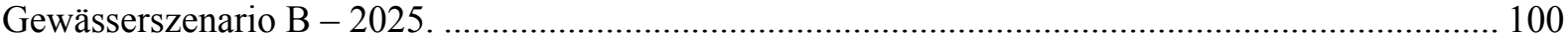

Abb. 94: Sensitivitätskarte Gewässerökologie. Hinterlegt mit den Besucherfrequenzen 2025 ......... 101 Abb. 95: Darstellung der ökologischen Funktionsfähigkeit der Gewässer für das Gewässerszenario A.

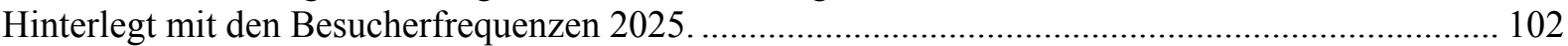
Abb. 96: Sensitivitätskarte Gewässerökologie für das Gewässerszenario B. Hinterlegt mit den Besucherfrequenzen 2025.

Abb. 97: Darstellung der ökologischen Funktionsfähigkeit der Gewässer für das Gewässerszenario B. Hinterlegt mit den Besucherfrequenzen 2025.

Abb. 98: Darstellung der zugrundeliegenden Daten (Gewässertiefe bei einer Donauwasserführung von $2.200 \mathrm{~m} 3 / \mathrm{s}$ und Biotoptypen) für die Erstellung einer Sensibilitätskarte für den Biber für das Szenario Lobau A (ohne Gewässerszenario 1b).

Abb. 99: Darstellung der zugrundeliegenden Daten (Gewässertiefe bei einem mittleren

Donaudurchfluss von $2.200 \mathrm{~m} 3 / \mathrm{s}$ und Biotoptypen) für die Erstellung einer Sensibilitätskarte für den Biber für das Szenario Lobau B (mit Gewässerszenario 1b).

Abb. 100: Darstellung der zugrundeliegenden Daten (Wegenetz inkl. Besucherzahlen, Wegbreite etc. und Biotoptypen) für die Erstellung einer Sensibilitätskarte für Reh- und Rotwild für das Szenario Lobau A (ohne Gewässerszenario 1b).

Abb. 101: Darstellung der zugrundeliegenden Daten (Wegenetz inkl. Besucherzahlen, Wegbreite etc. und Biotoptypen) für die Erstellung einer Sensibilitätskarte für Reh- und Rotwild für das Szenario Lobau B (mit Gewässerszenario 1b).

Abb. 102: Prozentueller Anteil der als unterschiedlich sensibel gegenüber Störung durch Besucher eingestuften Flächen in der Oberen und Unteren Lobau für den Biber; jeweils für das Szenario Lobau A (ohne Gewässerszenario 1b; links) und Lobau B (mit Gewässerszenario 1b; rechts). 1=nicht sensibel, 2=gering sensibel, 3=mäßig sensibel, 4=hoch sensibel, 5=sehr hoch sensibel. .................. 111 Abb. 103: Sensibilitätskarte für den Biber für das Szenario Lobau A (ohne Gewässerszenario 1b). Für die Badegewässer „Dechant Lacke“ und „Donau-Oder-Kanal“ waren keine Tiefenangaben vorhanden, weshalb für diese beiden Gewässer keine Sensibilität errechnet werden konnte. Sensibilitätsskala: keine (dunkelgrün) / gering (hellgrün) / mäßig (gelb) / hoch (orange) / sehr hoch (rot).................... 111 Abb. 104: Sensibilitätskarte für den Biber für das Szenario Lobau B (mit Gewässerszenario 1b). Für die Badegewässer „Dechant Lacke“ und „Donau-Oder-Kanal“ waren keine Tiefenangaben vorhanden, weshalb für diese beiden Gewässer keine Sensibilität errechnet werden konnte. Sensibilitätsskala: keine (dunkelgrün) / gering (hellgrün) / mäßig (gelb) / hoch (orange) / sehr hoch (rot).................... 112 Abb. 105: Prozentueller Anteil der als unterschiedlich sensibel gegenüber Störung durch Besucher eingestuften Flächen in der Oberen und Unteren Lobau für Reh- und Rotwild; jeweils für das Szenario Lobau A (ohne Gewässerszenario 1b; links) und Lobau B (mit Gewässerszenario ab; rechts). 1=nicht sensibel, 2=gering sensibel, $3=$ mäßig sensibel, $4=$ hoch sensibel, $5=$ sehr hoch sensibel.

Abb. 106: Sensibilitätskarte für Reh- und Rotwild für das Szenario Lobau A (ohne Gewässerszenario 1b). Sensibilitätsskala: keine (dunkelgrün) / gering (hellgrün) / mäßig (gelb) / hoch (orange) / sehr hoch (rot).

Abb. 107: Sensibilitätskarte für Reh- und Rotwild für das Szenario Lobau B (mit Gewässerszenario 1b). Sensibilitätsskala: keine (dunkelgrün) / gering (hellgrün) / mäßig (gelb) / hoch (orange) / sehr hoch (rot).

Abb. 108: Empfindlichkeit der Lobau (Sensibilität) gegenüber hohen Besucherströmen

Abb. 109: Überlagerung der Empfindlichkeit der Lobau (Sensibilität) mit den Nutzungsintensitäten eines Spitzentages

Abb. 110: Überlagerung der Empfindlichkeit der Lobau (Sensibilität) mit den Nutzungsintensitäten eines Spitzentages basierend auf Szenario „flächig ohne $1 b^{\text {“ }}$

Abb. 111: Veränderung der Verkehrsleistung durch Vekehrsszenarien. Quelle: ÖIR, eigene

Berechnung, Verkehrsmodell Wien-Ostregion. (PRM = Parkraummanagement)

Abb. 112: Veränderung der Verkehrsleistung durch Vekehrsszenarien. Quelle: ÖIR, eigene

Berechnung, Verkehrsmodell Wien-Ostregion. (PRM = Parkraummanagement) 
Abb. 113: Umweltwirkungen der Verkehrsszenarien. Quelle: ÖIR, eigene Berechnung,

Verkehrsmodell Wien-Ostregion. (PRM = Parkraummanagement)

\subsection{Tabellenverzeichnis}

Tab. 1: Entwicklungsetappen Masterplan Flugfeld Aspern. Quelle: Projektteam Flugfeld Aspern ....... 7

Tab. 2: Zuordnungsprinzip Entwicklungsetappen - Zeithorizonte Netzmodell ...................................... 7

Tab. 3: Einwohnerprognose 2004-2025. Quelle: ÖIR, Verkehrsmodell Wien. ....................................... 8

Tab. 4: Arbeitsplatzprognose. Quelle: ÖIR, Verkehrsmodell Wien..................................................... 9

Tab. 5: Einwohnerprognose. Quelle: ÖIR auf Grundlage Netzanalyse Wiener Linien 2007 sowie

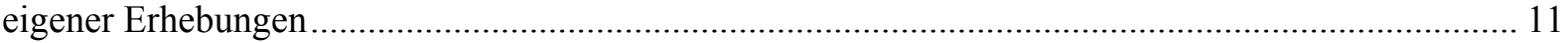

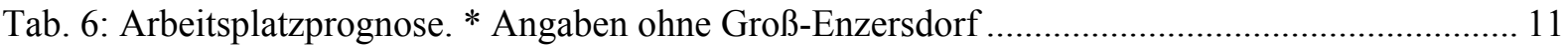

Tab. 7: Prognose der Besuchsintensitäten für die Lobau im Jahr 2025............................................. 12

Tab. 8: Definierte Szenarien als Maßnahmenbündel (1) 2025 fokussiert auf Radfahrende; (2)

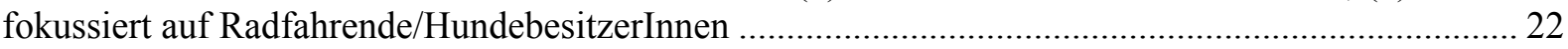

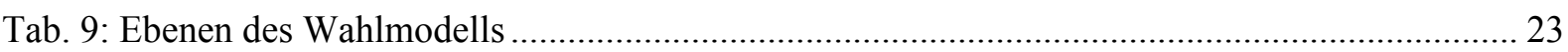

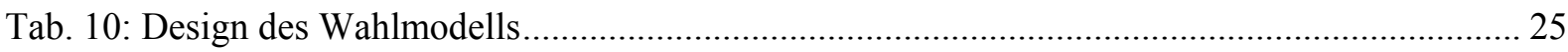

Tab. 11: Häufigkeit der ausgeübten Aktivitäten in der Lobau. ....................................................... 32

Tab. 12: Relative Wichtigkeit der dargestellten Attribute auf die einzelnen Aktivitäten $(\mathrm{N}=520)$...... 34

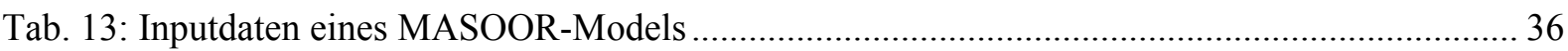

Tab. 14: Zusammenfassung der Kriterien, die die Routenwahl (Wegsegmentwahl) eines Agenten

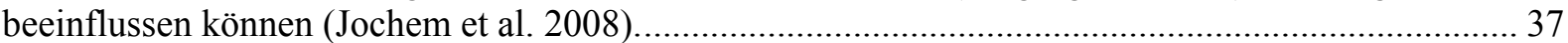

Tab. 15: Aufstellung der simulierten Managementszenarien (Lobau A = Lobau ohne Änderung; Lobau B = Lobau (Gewässer-Szenario „OPTIMA Lobau 1b“); Vorland A = lineare Ausgestaltung des LobauVorlandes; Vorland B = flächige Ausgestaltung des Lobau-Vorlandes) ............................................ 38

Tab. 16: Liste der thematischen Attribute des Wegenetzes............................................................... 41

Tab. 17: Prozentuelle Verteilung der Agententypen an einem Spitzentag und an einem Werktag....... 48 Tab. 18: Relative Wichtigkeit der MASOOR Bewegungsparameter für jede Agentenbewegungsphase (je höher ein Wert in der Tabelle, desto stärker ist der Einfluss eines Algorithmus; „, $0^{“}=$ Algorithmus

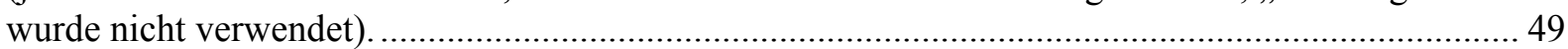

Tab. 19: MASOOR Inputparameter - Beispiele für Wegtyppräferenzen nach Agententypen .............. 50

Tab. 20: Wegelängen und Fortbewegungsgeschwindigkeit der LobaubesucherInnen als

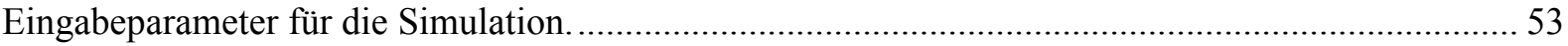

Tab. 21: Besucheraufkommen im Jahr 1999 (Arnberger et al., 2000) und Prognose des Gesamtbesucheraufkommens je Besuchstag für 2025 ..................................................................... 55

Tab. 22: Besucheraufkommen an einem Spitzen- und einem Werktag: beobachtete Besucherzahlen im Jahr 1999 und die Prognose für 2025 für zwölf Lobaueingänge........................................................ 56

Tab. 23: Aufstellung der simulierten Managementszenarien: Lobau A = Lobau ohne Änderung; Lobau B = Lobau (Gewässer-Szenario „OPTIMA Lobau 1b“); Vorland A = lineare Ausgestaltung des LobauVorlandes; Vorland B = flächige Ausgestaltung des Lobau-Vorlandes; Lobau A bedeutet ohne, Lobau

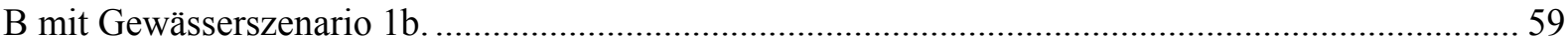

Tab. 24: Zusammenstellung der in der Vegetationskarte den IST-Zustand repräsentierenden Vegetationstypen (nach Reiter - Endbericht OPTIMA Lobau) ……................................................ 72

Tab. 25: Liste der auf Basis der Vegetationserhebung (Mayer 2007) ausgewiesenen Syntaxon........... 73

Tab. 26: Höhenabstufung in cm der einzelnen erarbeiteten Vegetationstypen .................................... 74

Tab. 27: Neunstufige Hemerobieskala und die fünf Stufen der Naturnähe (Grabherr et al., 1998)...... 76

Tab. 28: Hemerobiestufen der Stichprobenklassen bzw. der Waldbiotoptypen (aus Illedits \& Zsak, 2012)

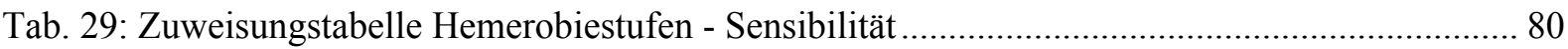

Tab. 30: Flächenanteile der Hemerobiestufen im Untersuchungsgebiet (aus Illedits \& Zsak, 2012) ... 82

Tab. 31: Aggregationstypen der in der Lobau beschriebenen Biotoptypen ......................................... 84 
Tab. 32: Größe der Aufnahmeflächen

Tab. 33: Syntaxone und klassenlose Vegetationsformationen beschrieben auf Basis des 375

Aufnahmen umfassenden Samples.

Tab. 34: Ergebnisse der multiplen linearen Regression für Makrophyten gesamt (Baart 2008)....

Tab. 35: Koeffizienten des statistischen Modells mit den Einflussvariablen Anteil der

Flachwasserbereiche (AntFWB), dem Hydrophytendeckungsgrad (KO_Hydr) und dem

logarithmierten Wert der Konnektivität (ln_con), $\mathrm{R}^{2}=0,66$.

Tab. 36: Nährstoffeintrag durch Badenutzung am Beispiel der drei Hauptbadegewässer der Unteren Lobau, Dechantlacke, Panozzalacke und Donau-Oder-Kanal. Eintrag pro Badegast 0,094 g pro Tag (Schulz 1981). Die Anzahl der Badegäste wurde aus den Nutzerfrequenzen an Spitzentagen der Szenarien 1999 und 2025 abgeleitet.

Tab. 37: Zusammenstellung der berechneten Indikatorwerte für die ökologische Funktionsfähigkeit.

Tab. 38: Schema für die Vergabe von Sensibilitäts-Punkten $(0=$ keine / $1=$ gering / $2=$ mäßig / 3=hoch /

$4=$ sehr hoch) für die verschiedenen Indikatoren für den Biber.

Tab. 39: Sensibilitätsskala für den Biber.

Tab. 40: Schema für die Vergabe von Sensibilitäts-Punkten $(0=$ keine / $1=$ gering / $2=$ mäßig / $3=$ hoch /

$4=$ sehr hoch) für die verschiedenen Indikatoren für Reh- und Rotwild.

Tab. 41: Sensibilitätsskala für Reh- und Rotwild.

Tab. 42: Indikatoren zur Bewertung der Sensibilität der Lobau (Skala: $1=$ nicht sensibel; $5=$ sehr hohe

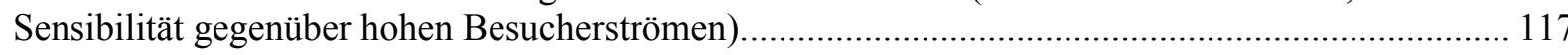

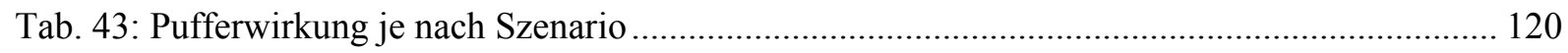

Tab. 44 : Anreiseverkehrsmittewahl der LobaubesucherInnen. Quelle: ÖIR, Hochrechnung der Erhebung 2000.

Tab. 45: Zusammenfassende Bewertung und Vergleich der vier Szenarien nach Fachbereichen ...... 128

Tab. 46: Rangreihung der Szenarien aus Sicht der Einzeldisziplinen (1=beste Reihung) 129 